
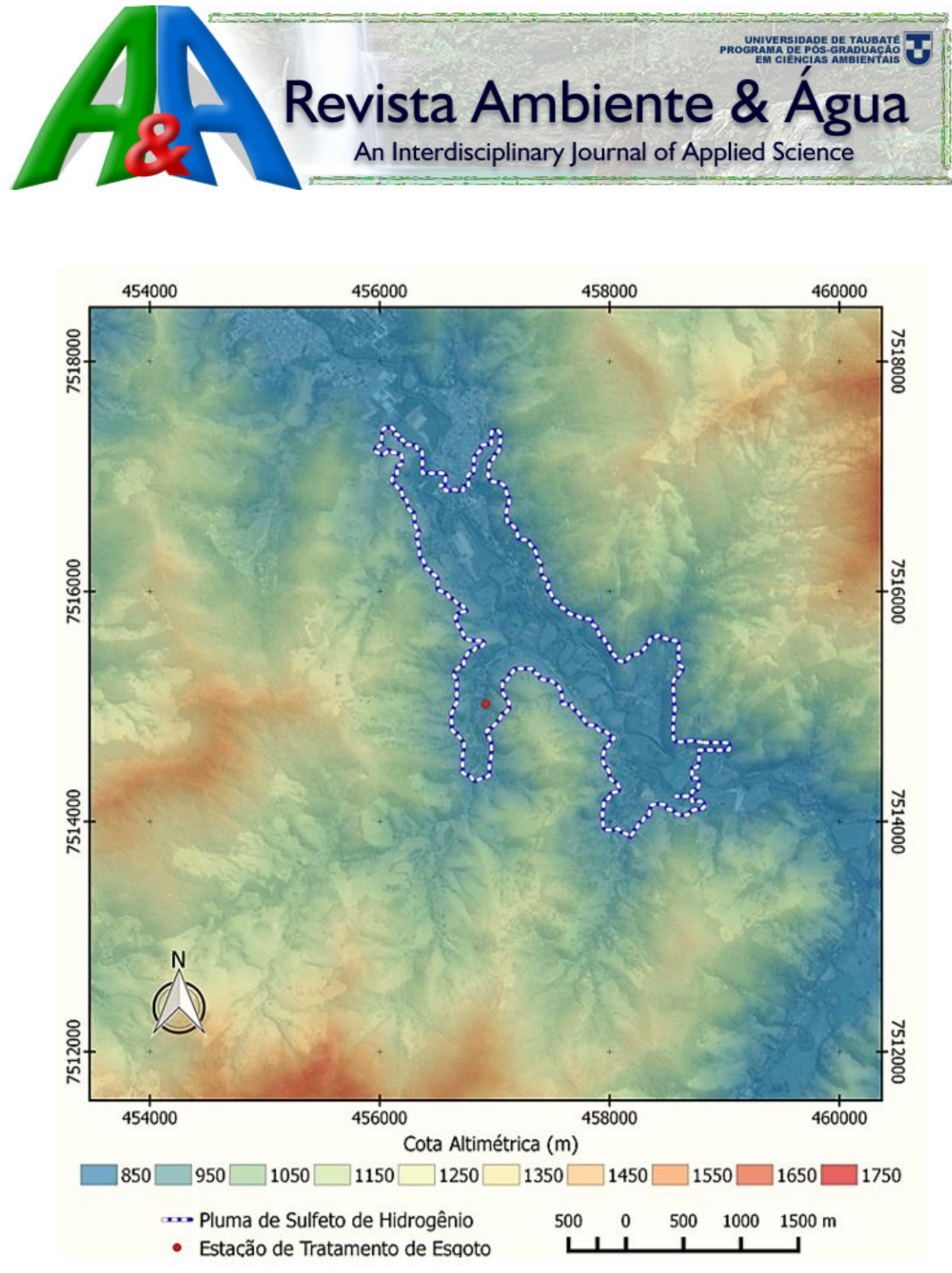

$\mathrm{N}=1980-993 X$ (Online) http://www.ambi-agua.net

$37^{\text {th }}$ Edition of Revista Ambiente \& Água - An Interdisciplinary Journal of Applied Science, Taubaté, V. 12, N.2, p. 168-350 Mar./Apr. 2017. (doi:10.4136/ambi-agua.v12.n2) 


\section{TABLE OF CONTENTS}

\section{COVER:}

\section{Influence of relief on $\mathrm{H}_{2} \mathrm{~S}$ dispersion.}

Source: AUGUSTO, M. R.et al. Simulação numérica da dispersão do sulfeto de hidrogênio emitido por um reator UASB para tratamento de esgoto doméstico. Rev. Ambient. Água, Taubaté, vol. 12 n. 2, p. 215-225, 2017. doi:10.4136/ambi-agua.1841

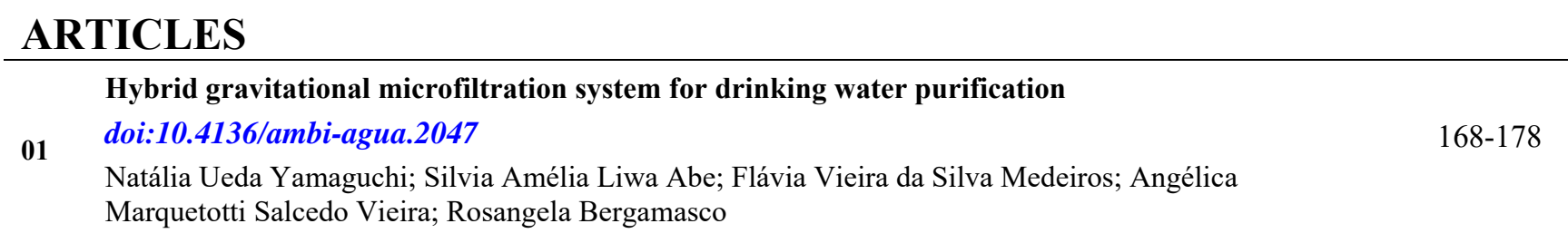

doi:10.4136/ambi-agua.1832

Narciso Paulino Junior; Rita de Cássia Silva von Randow; Celso von Randow

The use of caffeine as a chemical marker of domestic wastewater contamination in surface waters: seasonal and spatial variations in Teresópolis, Brazil

03

doi:10.4136/ambi-agua.1974

Eline Simões Gonçalves; Silvana Vianna Rodrigues; Emmanoel Vieira da Silva-Filho

\section{Emprego dos métodos expeditos de Chow Gumbel e Bell para formulação de equações de chuvas}

04 intensas - uma avaliação de desempenho

doi:10.4136/ambi-agua.2001

Karinnie Nascimento de Almeida; José Antonio Tosta dos Reis; Antônio Sergio Ferreira Mendonça

Simulação numérica da dispersão do sulfeto de hidrogênio emitido por um reator UASB para tratamento de esgoto doméstico

doi:10.4136/ambi-agua.1841

Matheus Ribeiro Augusto; Herlane Costa Calheiros;Vanessa Silveira Barreto Carvalho

Calibração e validação do modelo SWAT para simulação hidrológica em uma bacia hidrográfica do litoral norte catarinense

Éverton Blainski; Eileen Acosta; Patrícia Carvalho do Prado Nogueira

Caracterização estacional das variáveis físicas, químicas, biológicas e ecotoxicológicas em um trecho do Rio Paraíba do Sul, SP, Brasil

doi:10.4136/ambi-agua.1949

Lucas Gonçalves Queiroz; Flávio Teixeira da Silva, Teresa Cristina Brazil de Paiva

Ocorrência e remoção de estrogênios por processos de tratamento biológico de esgotos

08 doi:10.4136/ambi-agua.1992

Danieli Lima da Cunha; Lícia Murito de Paula; Samuel Muylaert Camargo da Silva; Daniele Maia Bila; Estefan Monteiro da Fonseca; Jaime Lopes da Mota Oliveira

Atributos físicos e estoque de carbono do solo em áreas de Terra Preta Arqueológica da Amazônia doi:10.4136/ambi-agua.1890

09 José Maurício da Cunha; Denilton Carlos Gaio; Milton César Costa Campos; Marcelo Dayron Rodrigues 
Diagnóstico espacial e temporal de condições físico-químicas e microbiológicas do Córrego do Tanquinho, Ribeirão Preto, SP, Brasil

10 doi:10.4136/ambi-agua.1837

Andre Abreu Grieco; Brisa Maria Fregonesi; Karina Aparecida de Abreu Tonani; Thaís Vilela Silva; Beatriz Smidt Celere; Tânia Maria Beltramini Trevilato; Susana Inés Segura-Muñoz; Renato Igor da Silva Alves

Gerenciamento de áreas contaminadas por postos de combustíveis em Cuiabá, Mato Grosso, Brasil

11 doi:10.4136/ambi-agua.1872

Suzy Darley de Lima; Andrea Ferreira de Oliveira; Rossean Golin; Danila Soares Caixeta; Zoraidy Marques de Lima; Eduardo Beraldo de Morais

Secas e seus impactos no município de Boqueirão, PB, Brasil

12 doi:10.4136/ambi-agua.2004

André Aires de Farias; Francisco de Assis Salviano de Sousa;João Miguel Moraes Neto; Anailson de

Sousa Alves

Avaliação do uso de extratos vegetais para controle da hemoncose em ovinos naturalmente infectados

13 doi:10.4136/ambi-agua.2020

Matheus Diniz Gonçalves Coêlho; Thalyta Baldim Xavier; Jaqueline Fabiana da Costa; Lucas Tobias Rodrigues Maciel;Lilian Saito Ormachea Bozo; Francine Alves da Silva Coêlho; Gokithi Akisue

Alexandre André Feil; Vírgilio José Strasburg; Fernando Rosado Spilki 


\section{EDITORIAL BOARD}

\section{Editor}

Getulio Teixeira Batista

Instituto de Pesquisas Ambientais em Bacias Hidrográficas (IPABHi), SP, BR

Amaury Paulo de Souza

Ana Aparecida da Silva Almeida

Andrea Giuseppe Capodaglio

Antonio Evaldo Klar

Antonio Teixeira de Matos

Apostol Tiberiu

Carlos Eduardo de M. Bicudo

Cláudia S. de C. M. dos S. Cordovil

Dar Roberts

Delly Oliveira Filho

Gabriel Constantino Blain

Giordano Urbini

Gustaf Olsson

Hélio Nobile Diniz

Ignacio Morell Evangelista,

János Fehér

João Vianei Soares

José Carlos Mierzwa

Julio Cesar Pascale Palhares

Luis Antonio Merino

Marcelo dos Santos Targa

Maria Cristina Collivignarelli,

Massimo Raboni

Nelson Wellausen Dias

Petr Hlavínek

Richarde Marques da Silva

Silvio Jorge Coelho Simões

Stefan Stanko

Teresa Maria Reyna

Yosio Edemir Shimabukuro

Zhongliang Liu Beijing

Editorial Section

Text Editors

Reference Editor

Layout Editors

Technical Support

Secretaria e Comunicação

\section{Associate Editors}

Universidade Federal de Viçosa (UFV), BR

Universidade de Taubaté (UNITAU), BR

University of Pavia, ITALY

Universidade Est. Paulista Júlio de Mesquita Filho (UNESP), BR

Universidade Federal de Viçosa (UFV), BR

University Politechnica of Bucharest, Romênia

Instituto de Botânica, IBT, BR

Centro de estudos de Engenharia Rural (CEER), Lisboa, Portugal

University of California, Santa Barbara, United States

Universidade Federal de Viçosa (UFV), BR

Instituto Agronômico de Campinas, IAC, Brasil.

University of Insubria, Varese, Italy

Lund University, Lund, Sweden

Inst. Geológico, Sec. do Meio Amb. do Est. de SP (IG/SMA),BR

University Jaume I- Pesticides and Water Research Institute, Spain

Debrecen University, Hungary

Instituto Nacional de Pesquisas Espaciais (INPE), BR

Universidade de São Paulo, USP, BR

Embrapa Pecuária Sudeste, CPPSE, São Carlos, SP, BR

Institute of Regional Medicine, National University of the Northeast, Corrientes, Argentina

Universidade de Taubaté, (UNITAU), BR

University of Pavia, Depart. of Civil Engineering and Architecture, Italy

LIUC - University "Cattaneo", School of Industrial Engineering, Italy

Inst. Bras. de Geogr. e Estatística (IBGE). Aracaju, SE, BR

Brno University of Technology República Tcheca

Universidade Federal da Paraíba, UFPB, BR

Univ. Est. Paulista Júlio de Mesquita Filho, UNESP, BR

Slovak Technical University in Bratislava Slovak, Eslováquia

Universidad Nacional de Córdoba, Argentina

Instituto Nacional de Pesquisas Espaciais (INPE), BR

University of Technology, China

Nelson Wellausen Dias, PPGCA, UNITAU, IBGE, BR

Theodore D`Alessio, FL, USA, Maria Cristina Bean, FL, USA

Getulio T. Batista, UNITAU, BR

Luciana Pedrosa da Fonseca Batista, - New Jersey, USA

Liliane Castro, Bibliotecária - CRB/8-6748, Taubaté, BR

Vera L. F. Batista, IPABHi \& Getulio T. Batista, UNITAU, BR

Tiago dos Santos Agostinho, LAGEO, UNITAU, BR

Luciana Gomes de Oliveira, UNITAU, BR

Library catalog entry by Liliane Castro CRB/8-6748

Revista Ambiente \& Água - An Interdisciplinary Journal of Applied Science / Instituto de Pesquisas

Ambientais em Bacias Hidrográficas. Taubaté. v. 12, n. 2 (2006) - Taubaté: IPABHi, 2017.

Quadrimestral (2006 - 2013), Trimestral (2014 - 2016), Bimestral a partir de Janeiro de 2017.

Resumo em português e inglês.

ISSN 1980-993X

1. Ciências ambientais. 2. Recursos hídricos. I. Instituto de Pesquisas Ambientais em Bacias Hidrográficas. 


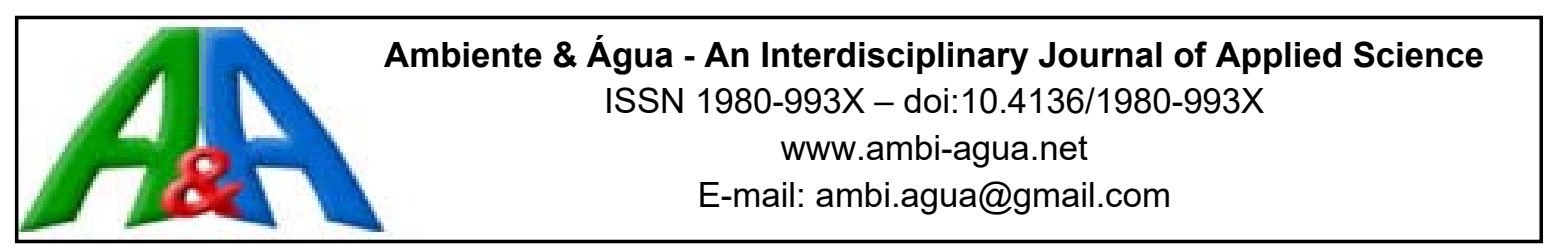

\title{
Hybrid gravitational microfiltration system for drinking water purification
}

\author{
doi:10.4136/ambi-agua.2047
}

Received: 12 Apr. 2016; Accepted: 19 Jan. 2017

\author{
Natália Ueda Yamaguchi ${ }^{1 *}$; Silvia Amélia Liwa $\mathrm{Abe}^{2}$; \\ Flávia Vieira da Silva Medeiros ${ }^{3}$; Angélica Marquetotti Salcedo Vieira ${ }^{2}$; \\ Rosangela Bergamasco ${ }^{2}$ \\ ${ }^{1}$ Centro Universitário de Maringá (Unicesumar), Maringá, PR, Brasil \\ Programa de Pós-Graduação em Tecnologias Limpas \\ ${ }^{2}$ Universidade Estadual de Maringá (UEM), Maringá, PR, Brasil \\ Departamento de Engenharia Química \\ ${ }^{3}$ Universidade Tecnológica Federal do Paraná (UTFPR), Campo Mourão, PR, Brasil \\ *Corresponding author: e-mail: nataliaueda@hotmail.com, \\ silvia_abe@hotmail.com, flaviavs@gmail.com, amsvieira@uem.br, \\ ro.bergamasco@hotmail.com
}

\begin{abstract}
This study evaluates the application of a polymeric microfiltration membrane in a gravitational filtration module and its combination with granular activated carbon (GAC) impregnated with copper, resulting in a hybrid process. The proposed system would be used to improve the quality of water for human consumption in developing countries. Permeate flux, $\mathrm{pH}$, Escherichia coli removal, color, turbidity and free chlorine removals were evaluated in the applied process. Instrumental techniques, such as $\mathrm{N}_{2}$ adsorption at $77 \mathrm{~K}$, scanning electron microscopy (SEM), transmission electron microscopy (TEM) and energy dispersive X-ray spectroscopy (EDX) analyses were used to characterize the proposed membrane and adsorbent. The GAC ensured higher chlorine removals, as well as higher permeate flux. Furthermore, the GAC impregnated with copper oxide nanoparticles exhibited higher Escherichia coli removal. Therefore, the hybrid gravitational membrane system applying GAC impregnated with copper oxide could be considered as a potential alternative point-of-use treatment to improve the quality of water for human consumption.
\end{abstract}

Keywords: activated carbon; copper; Escherichia coli; membrane.

\section{Sistema de microfiltração gravitacional híbrido para purificação de água}

\section{RESUMO}

Neste estudo, uma membrana polimérica combinada com carvão ativado granular (CAG) impregnado com nanopartículas de óxido de cobre foi avaliada em um processo híbrido com membrana. A avaliação do sistema gravitacional proposto visando a melhoria da qualidade da água destinada ao consumo humano em países em desenvolvimento foi realizada avaliando-se o fluxo permeado, pH, remoção de Escherichia coli, cor, turbidez e a remoção de cloro livre. Técnicas instrumentais tais como adsorção de $\mathrm{N}_{2}$ a $77 \mathrm{~K}$, microscopia eletrônica de varredura 
(MEV), microscopia eletrônica de transmissão (MET) e análises de espectroscopia de raios-X por dispersão em energia (EDX) foram utilizadas para caracterizar o sistema proposto de membrana e adsorvente. O CAG garantiu maiores remoções de cloro, e também maior fluxo permeado. Além disso, o CAG impregnado com nanopartículas de óxido de cobre exibiu maior remoção de E. coli. Portanto, o sistema de membrana gravitacional híbrido utilizando CAG impregnado com óxido de cobre pode ser considerado como um tratamento potencial alternativo pontual para melhorar a qualidade da água destinada ao consumo humano.

Palavras-chave: carvão ativado; cobre; Escherichia coli; membrana.

\section{INTRODUCTION}

Scientific advancements in water purification can aid the development of new technologies appropriate for different regions of the world. The sheer enormity of the problems that the world is facing due the lack of adequate clean water and sanitation means that much more work is needed to address the challenges faced by developing nations, which suffer a diversity of socioeconomical-political-traditional constraints, and require a broader approach incorporating sustainable energy sources and implementing educational and capacity building strategies. Consortiums of governments at all levels, businesses and industries, financial and health organizations, water and environment associations, and educational and research institutions need to focus increased attention on solving these problems. While better water resource management, improved efficiencies, and conservation are vital for moderating demand and improving availability, it is our belief that improving the science and technology of water purification can help to provide cost-effective and robust solutions (Shannon et al., 2008).

In developing countries, centralized drinking water treatment is sometimes deficient, leading to the frequent use of untreated natural water sources such as rivers, lakes, groundwater or rain. These sources are usually unprotected and may contain chemical or microbial pollutants, mainly originated from the absence of appropriate sanitation and thus contaminated by human and animal residues that are either active cases or carriers of disease. Sometimes, even when centralized treatment plants are available, contamination of piped drinking water may occur between the distribution system and the final consumption point (Peter-Varbanets et al., 2009). Also, in rural or informal urban or peri-urban areas there is no centralized water supply. Consequently, decentralized systems are often the only means of improving the quality of water obtained from contaminated sources (Peter-Varbanets et al., 2009).

Among these systems, membrane separation processes are suitable for water treatment since they can remove bacteria, viruses, turbidity and color (Bergamasco et al., 2011b). However, most membrane processes are pressure-driven and require pumps and high energy consumption. Because of the high cost of constructing large pressure vessels with the proper wetted surfaces for corrosion resistance, pressure filters are typically used for small- and medium-size capacity water reverse osmosis plants. Gravity filters are used for all sizes of water reverse osmosis in water treatment plants but have found wider applications for large and medium-size facilities (Voutchkov, 2010). Other researchers have reported that the disadvantages of membrane water treatment are fouling and plugging. Its occurrence leads to a decline in membrane permeability and reduced flux (Kim et al., 2009).

Integration of a pretreatment with a membrane filtration process has been employed to reduce membrane fouling and to improve the removal of certain contaminants. It is reported that the combination of activated carbon with membrane filtration increased the obtained volume of filtrated water and prolonged the continuous filtration time by mitigating membrane fouling (Ajmani et al., 2012; Kim et al., 2009; Silva et al., 2012).

Moreover, it is well known that activated carbon shows strong adsorbability of several kinds of organic pollutants, trace heavy metal ions and some nonmetal ions due to its 
microporous structure and immense specific surface. If copper can be loaded on the surface of activated carbon with high specific surface area, it may enlarge the reaction area between copper and ions/organic molecules and effectively improve the efficiency of the adsorbent (Deveci et al., 2006; Liu et al., 2011; Zhao et al., 2010). In addition, copper presents bactericidal properties, as reported in some studies (Castro et al., 2010; Moritz e Geszke-Moritz, 2013). They have reported $\mathrm{Cu}$-textiles and composite materials presenting antiviral, algicide and fungicide properties. Copper-impregnated materials have been reported to be effective against E. coli, Staphylococcus aureus and the fungi Candida albicans and were also effective against other infections, besides being safe to humans (as demonstrated by the widespread and prolonged use by women of copper intrauterine devices) and not causing skin irritation when externally applied (Borkow e Gabbay, 2004; Castro et al., 2010).

The main objective of the study was to propose a simple module that ensures water quality for human consumption in a simple and inexpensive household filter destined for developing countries.

\section{MATERIALS AND METHODS}

\subsection{Activated carbon modification}

This study used commercial GAC 16 x 52 mesh obtained from oil palm shell supplied by BAHIACARBON (Brazil). Copper oxide nanoparticles were impregnated on the porous GAC using a wet technique. $150 \mathrm{~g}$ of GAC were added to $150 \mathrm{ml}$ of an aqueous solution of copper sulfate in order to obtain 0.5 or $1.0 \mathrm{wt} . \%$ of $\mathrm{Cu}$ loading. The solution was stirred in a rotary evaporator system for $24 \mathrm{~h}$ at $60^{\circ} \mathrm{C}$ and $20 \mathrm{rpm}$. Afterwards, it was dried at $80^{\circ} \mathrm{C}$ in a vacuum system in the same equipment. The samples were successively calcined at $350^{\circ} \mathrm{C}$ for $5 \mathrm{~h}$ (Bergamasco et al., 2010). Samples with 0.5 and 1.0 wt.\% of $\mathrm{Cu}$ loading were named $\mathrm{GAC} / \mathrm{Cu} 05$ and $\mathrm{GAC} / \mathrm{Cu} 10$, respectively.

\subsection{Characterization}

GAC pore characteristics (surface area, pore volume and average pore size) were measured in a Quantachrome Autosorb Automated Gas Sorption System (Florida, USA). BET specific surface areas were determined by $\mathrm{N}_{2}$ adsorption-desorption isotherms at $77 \mathrm{~K}$. Micropore area was determined by means of the t-method of Halsey and mesopore area, volume, average pore diameter using Barrett-Joyner-Halenda method (BJH). Volume and average pore diameter of micropores were calculated using Horvath-Kawazoe method (HK).

The surface morphology and composition of new membrane and GAC before use were studied using a scanning electron microscope (SEM) FEI (Model Nova 200 NanoSEM), energy dispersive X-ray spectroscopy (EDX). The morphology of copper oxide nanoparticles was also analyzed by transmission electron microscopy (TEM).

\subsection{Hybrid membrane gravitational system}

Figure 1 shows the gravitational filtration module used in this study, which was proposed in a previous study (Silva et al., 2012).

The cellulose acetate microfiltration membrane (pore diameter $=3,0 \mu \mathrm{m}$ ) was purchased from ADVANTEC (Japan). GAC impregnated with copper oxide nanoparticles was placed in the filtration module, before the flat membrane, as a pre-treatment in a packed-bed filter resulting in a hybrid gravitational system as shown in Figure 1A. This filter was obtained by packing $150 \mathrm{~g}$ of GAC in a $20-\mathrm{cm}$ high acrylic cartridge. Additional tests were carried out to compare the effects of GAC pretreatment with the unitary process, which was the process using membrane alone, illustrated in Figure 1B. All process conditions evaluated in the gravitational system are given in Table 1. 


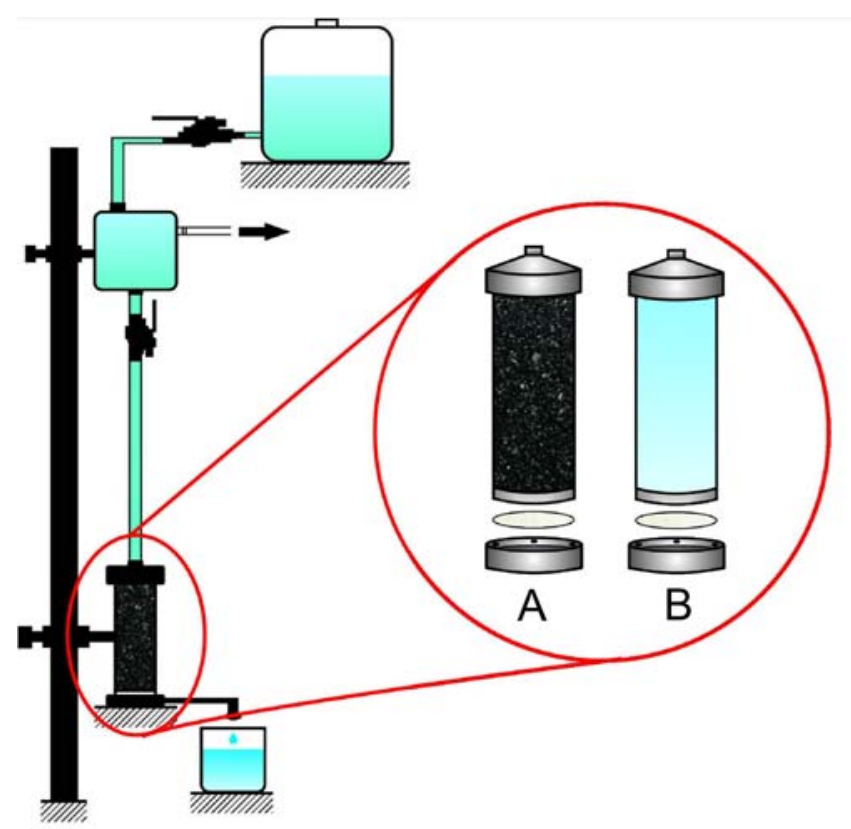

Figure 1. Scheme of hybrid gravitational system (A) and unitary process (B).

Table 1. Process conditions evaluated in this study.

\begin{tabular}{ll}
\hline Abbreviation & \multicolumn{1}{c}{ Evaluated process } \\
\hline $\mathrm{M}$ & Unitary membrane process \\
$\mathrm{M}+\mathrm{GAC}$ & Hybrid process (membrane and plain GAC) \\
$\mathrm{M}+\mathrm{GAC} / \mathrm{Cu} 05$ & Hybrid process (membrane and GAC impregnated with $0.5 \%$ wt. $\mathrm{Cu}$ ) \\
$\mathrm{M}+\mathrm{GAC} / \mathrm{Cu} 10$ & Hybrid process (membrane and GAC impregnated with $1.0 \%$ wt. $\mathrm{Cu}$ ) \\
\hline
\end{tabular}

Each process system was evaluated performing 5 steps according to previous studies (Bergamasco et al., 2011a; Silva et al., 2012): (1) determination of the initial permeate flux with deionized water with a clean membrane, (2) filtration of deionized water contaminated with E. coli with an approximate concentration of $1 \times 10^{5} \mathrm{CFU} \mathrm{mL}^{-1}$ (3) four subsequent filtrations of tap water during $120 \mathrm{~min}$ totalizing $480 \mathrm{~min}$ of filtration, (3) second filtration of water artificially contaminated with E. coli, as described in step 2 . This test was carried out to evaluate the final E. coli removal, after tap water filtration (5) determination of final permeate flux of deionized water with dirty membrane.

\subsection{Water samples and analysis}

Tests of Escherichia coli and chlorine removal, color, turbidity, $\mathrm{pH}$ variation, flux and total volume of filtrated tap water were performed to characterize the filtration system efficiency. All procedures were carried out according to Standard Methods for the examination of water and wastewater (APHA et al., 2012). Two types of water were used, deionized water artificially contaminated with E. coli and tap water. The characteristics of tap water used in this study are presented in Table 2 . 
Table 2. Characteristics of tap water.

\begin{tabular}{lc}
\hline \multicolumn{1}{c}{ Parameter } & Value \\
\hline Color (HU) & 0 \\
Turbidity (NTU) & $0.09-1.21$ \\
Free Chlorine $\left(\mathrm{mg} \mathrm{L}^{-1}\right)$ & $0.02-0.63$ \\
$\mathrm{pH}$ & $6.86-7.51$ \\
\hline
\end{tabular}

Removal of color, turbidity, free chlorine and $\mathrm{pH}$ variation were also measured during filtration. Color and free chlorine were measured with a HACH DR/4000 spectrophotometer and turbidity was measured using a $\mathrm{HACH} 2100 \mathrm{~N}$.

After the filtration of contaminated water, E. coli removal was evaluated using the membrane filter technique for members of the coliform group, as described in the Standard methods for the examination of water and wastewater (APHA et al., 2012).

\section{RESULTS AND DISCUSSION}

\subsection{Materials characterization}

SEM images of a new membrane and GAC before use are shown in Figure 2.

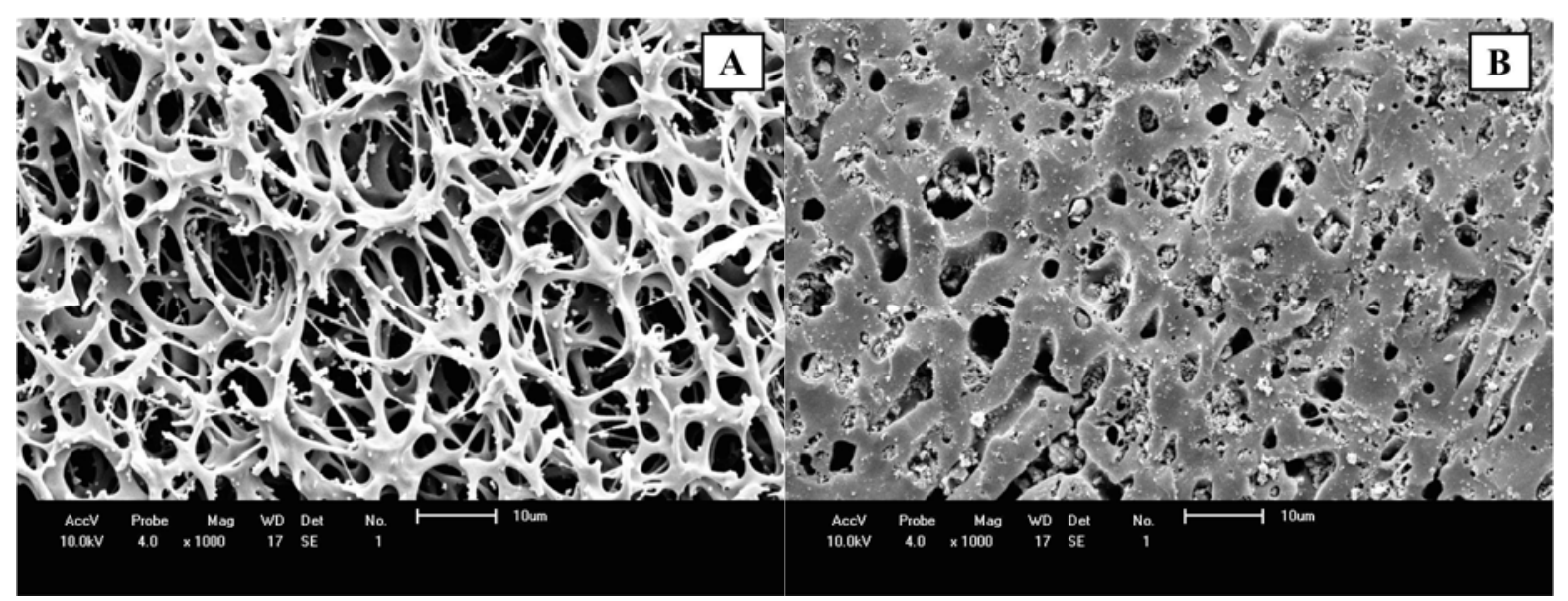

Figure 2. SEM images of evaluated membrane (A) and GAC (B).

Wide $(2-10 \mu \mathrm{m})$ and irregular pores are observed in Figure 2A from SEM membrane micrographs. GAC micrographs also showed wide macropores with a flat morphology surface in Figure 2B. Similar micrographs were reported by other authors (Jia and Lua, 2008; Ngarmkam et al., 2011).

From the EDX results for GAC and GAC impregnated with copper oxide, it was determined that the GAC composition is carbon and silica. The presence of silica in activated carbon was also reported by others authors (Giri et al., 2012; Haro et al., 2012). Regarding impregnated samples, EDX analysis confirms the presence of copper. Also, the EDX spectrum of GAC did not show the presence of oxygen, although the presence of oxygen is noticeable in impregnated samples. These results indicate the presence of copper oxide nanoparticles in activated carbon (Ruparelia et al., 2008).

Figure 3 presents the result of TEM analysis of GAC impregnated with copper oxide nanoparticles. 


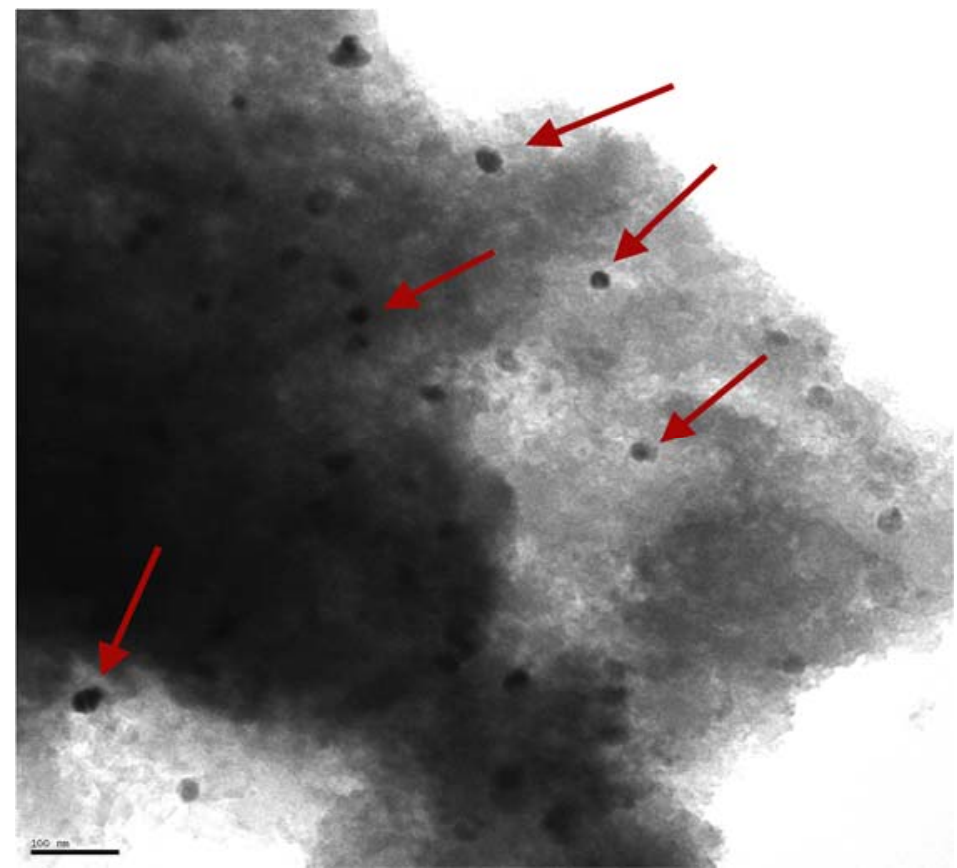

Figure 3. TEM micrograph analysis of GAC impregnated with copper nanoparticles.

The presence of copper was confirmed again, with arrows indicating copper oxide nanoparticles in GAC matrix of regular spherical sizes of approximately $20 \mathrm{~nm}$. Similar results were obtained by other authors using carbon matrix and copper (Khare et al., 2014; Singh et al., 2013).

The results of textural GAC characterization are presented in Table 3.

Table 3. Textural characteristics of the activated carbon used in this study.

\begin{tabular}{lccccccc}
\hline & \multicolumn{3}{c}{ Area $\left(\mathrm{m}^{2} \mathrm{~g}^{-1}\right)$} & \multicolumn{2}{c}{ Volume $\left(\mathrm{cm}^{3} \mathrm{~g}^{-1}\right)$} & \multicolumn{2}{c}{ Diameter $(\AA)$} \\
\cline { 2 - 7 } & BET & Micro & Meso & Micro & Meso & Micro & Meso \\
\hline GAC & 824 & 745 & 28 & 0.41 & 0.04 & 14 & 35 \\
GAG/Cu05 & 792 & 727 & 16 & 0.40 & 0.02 & 14 & 35 \\
GAC/Cu10 & 758 & 696 & 20 & 0.38 & 0.03 & 15 & 35 \\
\hline
\end{tabular}

GAC used in this study showed a predominantly microporous characteristic by $\mathrm{N}_{2}$ adsorption-desorption analysis. Comparing BET area from GAC and GAC/Cu samples, a decrease in this parameter was observed in impregnated samples as well as in the volume of micropores and mesopores. These results indicate that the impregnation of copper oxide lead to the lowering of their surface pores and volume. These phenomena are caused because pore blocking is a result of copper and carbon matrix interaction (Goscianska et al., 2012).

Both the volumes of micropores and mesopores were reduced after modifications, suggesting that copper oxide nanoparticles are located in both mesopores and micropores (Bandosz e Petit, 2009).

\subsection{Gravitational System Evaluation}

Figure 4 presents of all the evaluated parameters after the filtration of tap water through the filtration system and the initial and final flux for all the processes studied. 

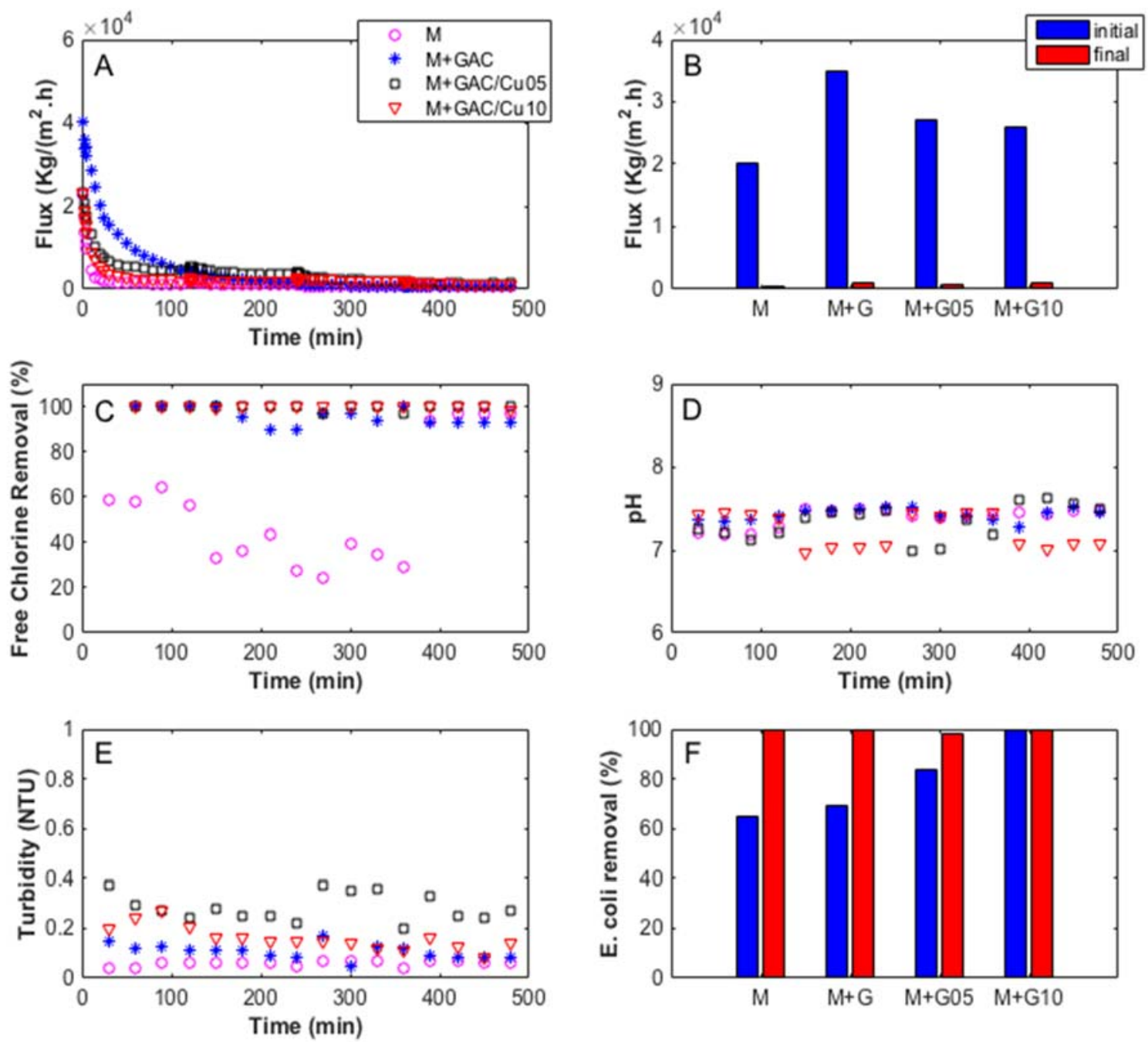

Figure 4. Permeate flux of tap water in filtration system (A), initial and final flux (B), and evaluated parameters of free chlorine removal (C), $\mathrm{pH}(\mathrm{D})$, turbidity (E), and E. coli removal (F) for all filtration systems studied.

Discontinuities were observed, since the filtration was carried out in four sequential steps of $120 \mathrm{~min}$ each, comprising $480 \mathrm{~min}$. Moreover, Figure 4B revealed a higher flux in hybrid system with GAC not impregnated with copper nanoparticles. These results showed that the flux obtained in the system filtration with GAC is greater than the one in system filtration without GAC, and that the flux decay is faster in the process without GAC. Thus, GAC ensured a higher flux and lower membrane fouling. This is possibly the result of the reduction of contaminants loaded onto the membrane due the adsorption of contaminants on the GAC (Kim et al., 2009).

Textural analysis showed that GAC impregnated with copper nanoparticles has smaller specific area due to pore blocking, thus presenting decreased adsorbent rate when compared with GAC without copper impregnation. Consequently, GAC without copper impregnation seems to decrease membrane fouling more efficiently and increases water flux. To confirm that theory, more specific adsorption studies with the GAC and GAC impregnated with copper nanoparticles must be done.

All samples showed the same color $0 \mathrm{HU}$. As this parameter seemed unvarying, the conclusion that can be drawn with this result is that the hybrid gravitational filtration system did not add color to the filtered water in any of the studied systems. According to Figure 4D, 
the filtered water presented a $\mathrm{pH}$ close to neutrality, as well as tap water. Therefore, the filtration system did not change the $\mathrm{pH}$, even though it is known that activated carbon causes $\mathrm{pH}$ raise during water treatment (Farmer et al., 1996). This result can be explained by the rapid filtration system with low hydraulic retention time, which does not allow high contact time.

The percentage of removal of turbidity showed an extensive range, from 25 to $82 \%$. However, the turbidity of tap water was not lowered by the filtration system; it is notable that turbidity values are higher when hybrid systems are used. This may be due to the fact that fine particles of GAG are solubilized. The hybrid filtration system with activated carbon significantly increased chlorine removal, higher than $90 \%$ in all collected samples, while the filtration system for the unitary process without GAC showed low chlorine removals, confirming that microfiltration itself does not remove some water contaminants and that activated carbon can be used as a combined technology to improve water quality (Kim et al., 2009).

Regarding the final E. coli removal presented in Figure 4F, all filtration systems obtained a $99.99 \%$ of final E. coli removal, perhaps due the membrane fouling after tap water filtration. Initial E. coli removal, however, was noticeably increased in hybrid filtration systems according to the increase in copper concentration. Copper with $1.0 \mathrm{wt} . \%$ concentration was the only filtration system that exhibited bactericidal efficiency of $99.99 \%$, as observed in the initial assay. The bactericidal effect was expected owing to the presence of copper nanoparticles, confirmed by TEM analysis (Figure 3), and according to the experimental results obtained by many authors in studies of the antibacterial effect of copper nanoparticles (Ruparelia et al., 2008).

\section{CONCLUSIONS}

The results of this study showed that is possible to enhance the quality of tap water in a hybrid gravitational membrane system, driven without requiring an external energy supply, such as pumps. GAC ensured higher chlorine removals, as well as higher initial permeate fluxes. GAC impregnated with copper also increased bactericidal efficiency. Therefore, the hybrid gravitational membrane system, using GAC impregnated with $1 \mathrm{wt} . \%$ of copper, could be considered as an alternative and inexpensive point-of-use treatment of water destined for human consumption in emerging countries.

\section{ACKNOWLEDGMENTS}

The authors would like to thank the Conselho Nacional de Desenvolvimento Científico e Tecnológico (CNPq) for the Master's scholarship granted and the Instituto Cesumar de Ciência, Tecnologia e Inovação (ICETI) for supporting this project.

\section{REFERENCES}

AJMANI, G. S.; GOODWIN, D.; MARSH, K.; FAIRBROTHER, D. H.; SCHWAB, K. J.; JACANGELO, J. G.; HUANG, H. Modification of low pressure membranes with carbon nanotube layers for fouling control. Water Research, v. 46, n. 17, p. 5645-5654, 2012. http://dx.doi.org/10.1016/j.watres.2012.07.059

APHA; AWWA; WEF. Standard Methods for the Examination of Water \& Wastewater. 22 ed. Washington, DC, , 2012. 1496 p. 
BANDOSZ, T. J.; PETIT, C. On the reactive adsorption of ammonia on activated carbons modified by impregnation with inorganic compounds. Journal of Colloid and Interface Science, v. 338, n. 2, p. 329-345, 2009. http://dx.doi.org/10.1016/j.jcis.2009.06.039

BERGAMASCO, R. et al. Carvão ativado impregnado com prata e cobre para eliminação de microrganismos da água. PI 1000304-5. 28 jan. 2010.

BERGAMASCO, R.; DA SILVA, F. V.; ARAKAWA, F. S.; YAMAGUCHI, N. U.; REIS, M. H. M.; TAVARES, C. J. et al. Drinking water treatment in a gravimetric flow system with $\mathrm{TiO} 2$ coated membranes. Chemical Engineering Journal, v. 174, n. 1, p. 102-109, 2011a. http://dx.doi.org/10.1016/j.cej.2011.08.056

BERGAMASCO, R.; KONRADT-MORAES, L. C.; VIEIRA, M. F.; FAGUNDES-KLEN, M. R.; VIEIRA, A. M. S. Performance of a coagulation-ultrafiltration hybrid process for water supply treatment. Chemical Engineering Journal, v. 166, n. 2, p. 483-489, 2011 b. http://dx.doi.org/10.1016/j.cej.2010.10.076

BORKOW, G.; GABBAY, J. Putting copper into action: copper-impregnated products with potent biocidal activities. The FASEB Journal, v. 18, n. 14, p. 1728-1730, 2004. http://dx.doi.org/10.1096/fj.04-2029fje

CASTRO, C.; SANJINES, R.; PULGARIN, C.; OSORIO, P.; GIRALDO, S. A.; KIWI, J. Structure-reactivity relations for DC-magnetron sputtered $\mathrm{Cu}$-layers during E. coli inactivation in the dark and under light. Journal of Photochemistry and Photobiology A: Chemistry, v. 216, n. 2-3, p. 295-302, 2010.

http://dx.doi.org/10.1016/j.jphotochem.2010.06.030

DEVECI, H.; YAZICI, E. Y.; ALP, I.; USLU, T. Removal of cyanide from aqueous solutions by plain and metal-impregnated granular activated carbons. International Journal of Mineral Processing, v. 79, n. 3, p. 198-208, 2006.

http://dx.doi.org/10.1016/j.minpro.2006.03.002

FARMER, R. W.; DUSSERT, B. W.; KOVACIC, S. L. Improved granular activated carbon for the stabilization of wastewater $\mathrm{pH}$. American Chemical Society, Division of Fuel Chemistry, v. 41, n. 1, p. 456-460, 1996.

GIRI, A. K.; PATEL, R.; MANDAL, S. Removal of Cr (VI) from aqueous solution by Eichhornia crassipes root biomass-derived activated carbon. Chemical Engineering Journal, v. 185-186, n. 0, p. 71-81, 2012. http://dx.doi.org/10.1016/j.cej.2012.01.025

GOSCIANSKA, J.; NOWAK, I.; NOWICKI, P.; PIETRZAK, R. The influence of silver on the physicochemical and catalytic properties of activated carbons. Chemical Engineering Journal, v. 189-190, n. 0, p. 422-430, 2012. http://dx.doi.org/10.1016/j.cej.2012.02.069

HARO, M.; RUIZ, B.; ANDRADE, M.; MESTRE, A. S.; PARRA, J. B.; CARVALHO, A. P. et al. Dual role of copper on the reactivity of activated carbons from coal and lignocellulosic precursors. Microporous and Mesoporous Materials, v. 154, n. 0, p. 6873, 2012. http://dx.doi.org/10.1016/j.micromeso.2011.07.005 
JIA, Q.; LUA, A. C. Effects of pyrolysis conditions on the physical characteristics of oil-palmshell activated carbons used in aqueous phase phenol adsorption. Journal of Analytical $\begin{array}{lllllll}\text { and Applied Pyrolysis, v. 83, n. 2, p. 175-179, } 2008 . & \text {. }\end{array}$ http://dx.doi.org/10.1016/j.jaap.2008.08.001

KHARE, P.; SHARMA, A.; VERMA, N. Synthesis of phenolic precursor-based porous carbon beads in situ dispersed with copper-silver bimetal nanoparticles for antibacterial applications. Journal of Colloid and Interface Science, v. 418, n. 0, p. 216-224, 2014. http://dx.doi.org/10.1016/j.jcis.2013.12.026

KIM, K.-Y.; KIM, H.-S.; KIM, J.; NAM, J.-W.; KIM, J.-M.; SON, S. A hybrid microfiltrationgranular activated carbon system for water purification and wastewater reclamation/reuse. Desalination, v. 243, n. 1-3, p. 132-144, 2009. http://dx.doi.org/10.1016/j.desal.2008.04.020

LIU, H.; LIU, W.; ZHANG, J.; ZHANG, C.; REN, L.; LI, Y. Removal of cephalexin from aqueous solutions by original and $\mathrm{Cu}(\mathrm{II}) / \mathrm{Fe}(\mathrm{III})$ impregnated activated carbons developed from lotus stalks Kinetics and equilibrium studies. Journal of Hazardous Materials, v. 185, n. 2-3, p. 1528-1535, 2011. http://dx.doi.org/10.1016/j.jhazmat.2010.10.081

MORITZ, M.; GESZKE-MORITZ, M. The newest achievements in synthesis, immobilization and practical applications of antibacterial nanoparticles. Chemical Engineering Journal, v. 228, n. 0, p. 596-613, 2013. http://dx.doi.org/10.1016/j.cej.2013.05.046

NGARMKAM, W.; SIRISATHITKUL, C.; PHALAKORNKULE, C. Magnetic composite prepared from palm shell-based carbon and application for recovery of residual oil from POME. Journal of Environmental Management, v. 92, n. 3, p. 472-9, 2011. http://dx.doi.org/10.1016/j.jenvman.2010.08.031

PETER-VARBANETS, M.; ZURBRÜGG, C.; SWARTZ, C.; PRONK, W. Decentralized systems for potable water and the potential of membrane technology. Water Research, v. 43, n. 2, p. 245-265, 2009. http://dx.doi.org/10.1016/j.watres.2008.10.030

RUPARELIA, J. P.; CHATTERJEE, A. K.; DUTTAGUPTA, S. P.; MUKHERJI, S. Strain specificity in antimicrobial activity of silver and copper nanoparticles. Acta Biomater, v. 4, n. 3, p. 707-16, 2008. http://dx.doi.org/10.1016/j.actbio.2007.11.006

SHANNON, M. A.; BOHN, P. W.; ELIMELECH, M.; GEORGIADIS, J. G.; MARINAS, B. J.; MAYES, A. M. Science and technology for water purification in the coming decades. Nature, v. 452, n. 7185, p. 301-310, 2008.

SILVA, F. V.; YAMAGUCHI, N. U.; LOVATO, G. A.; SILVA, F. A.; REIS, M. H.; AMORIM, M. T. et al. Effects of coconut granular activated carbon pretreatment on membrane filtration in a gravitational driven process to improve drinking water quality. Environmental Technology, v. 33, n. 4-6, p. 711-6, 2012. http://dx.doi.org/10.1080/09593330.2011.589133

SINGH, S.; ASHFAQ, M.; SINGH, R. K.; JOSHI, H. C.; SRIVASTAVA, A.; SHARMA, A. et al. Preparation of surfactant-mediated silver and copper nanoparticles dispersed in hierarchical carbon micro-nanofibers for antibacterial applications. N Biotechnol, v. 30, n. 6, p. 656-65, 2013. http://dx.doi.org/10.1016/j.nbt.2013.05.002 
VOUTCHKOV, N. Considerations for selection of seawater filtration pretreatment system. Desalination, v. 261, n. 3, p. 354-364, 2010.

http://dx.doi.org/10.1016/j.desal.2010.07.002

ZHAO, Z.; XIAO, L.; SUN, F.; HUO, G.; LI, H. Study on removing mo from tungstate solution by activated carbon loaded with copper. International Journal of Refractory Metals and Hard Materials, v. 28, n. 4, p. 503-507, 2010.

http://dx.doi.org/10.1016/j.ijrmhm.2010.02.008 


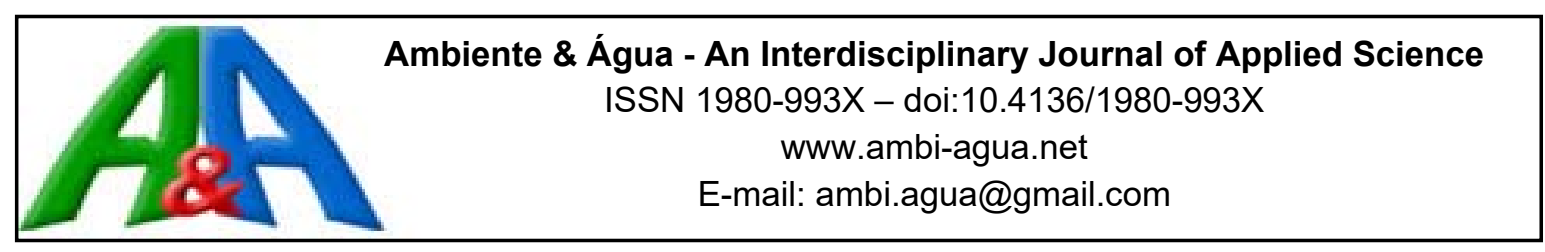

\title{
Analysis of biological and meteorological controls of evapotranspiration in pristine forests and a pasture site in Amazonia
}

\author{
doi:10.4136/ambi-agua.1832
}

Received: 11 Jan. 2016; Accepted: 17 Jan. 2017

\author{
Narciso Paulino Junior ${ }^{*}$; Rita de Cássia Silva von Randow ${ }^{2}$; Celso von Randow ${ }^{3}$ \\ ${ }^{1}$ Instituto Nacional de Pesquisas Espaciais (INPE), Cachoeira Paulista, SP, Brasil \\ Centro de Ciência do Sistema Terrestre (CCST) \\ ${ }^{2}$ Wageningen University and Research Centre (WUR), Wageningen, the Netherlands \\ Earth System Science and Climate Change (ESS-CC) \\ ${ }^{3}$ Instituto Nacional de Pesquisas Espaciais (INPE), São José dos Campos, SP, Brasil \\ Centro de Ciência do Sistema Terrestre (CCST) \\ "Corresponding author: e-mail: narciso.paulino@inpe.br, \\ rita.vonrandow@gmail.com, celso.vonrandow@inpe.br
}

\begin{abstract}
This work studied the behavior and seasonality of evapotranspiration influenced by biotic and abiotic factors through analysis of diurnal variation of aerodynamic resistance $\left(\mathrm{r}_{\mathrm{a}}\right)$, stomatal resistance $\left(\mathrm{r}_{\mathrm{s}}\right)$ and decoupling factor $(\Omega)$. This index was proposed by Jarvis and McNaughton (1986) as an indicative of the control of these resistances on the evapotranspiration of vegetation. Selection of representative data from wet and dry seasons from a primary forest in Central Amazonia and a primary forest and a pasture sites in Southwestern Amazonia had shown that: (i) $r_{a}$ is about $20 \mathrm{s.m}^{-1}$ in both forests in both seasons, and ranges from 70 to $100 \mathrm{~s}^{-1} \mathrm{~m}^{-1}$ in the pasture site; (ii) $\mathrm{r}_{\mathrm{s}}$ varies both throughout the day and seasonally, with medians increasing from 40 in the morning, to $150 \mathrm{s.m}^{-1}$ in late afternoon, in the wet season in the forests and from 50 to $160 \mathrm{s.m}^{-1}$ in the pasture. These values increase in the dry season, with the forests $\mathrm{r}_{\mathrm{s}}$ ranging from 50 up to $500 \mathrm{~s}^{-1} \mathrm{~m}^{-1}$ and pasture $\mathrm{r}_{\mathrm{s}}$ starting from $140 \mathrm{~s} . \mathrm{m}^{-1}$ and reaching up to more than $1800 \mathrm{s.m} \mathrm{m}^{-1}$ in the dry afternoons; (iii) $\Omega$ ranges from 0.5 to 0.8 during the wet season, and reduces to values below 0.5 in the afternoons during the dry season, indicating that, although a strong influence of net radiation in the evaporative loss is present, to a large extent the evapotranspiration fluxes are coupled to the biotic control of stomatal closure in the vegetation, especially in the pasture and during dry periods.
\end{abstract}

Keywords: aerodynamic resistance, decoupling factor, stomatal resistance.

\section{Análise de controles biológicos e meteorológicos da evapotranspiração em áreas de florestas primárias e pastagem na Amazônia}

\section{RESUMO}

O comportamento e a sazonalidade da evapotranspiração influenciada por fatores bióticos e abióticos foram estudados por meio da análise de variações diárias da resistência aerodinâmica $\left(\mathrm{r}_{\mathrm{a}}\right)$, da resistência estomática $\left(\mathrm{r}_{\mathrm{s}}\right)$ e do fator de desacoplamento $(\Omega)$, um índice proposto por Jarvis e McNaughton (1986) como um indicativo do controle das resistências na evapotranspiração da vegetação. A seleção de dados representativos de estações secas e úmidas de uma floresta primária na Amazônia Central, uma floresta primária e uma área de pastagem 
no sudoeste da Amazônia, demonstrou que: (i) ra é cerca de $20 \mathrm{~s} . \mathrm{m}^{-1}$ nas florestas em ambas as estações, e varia de 70 a $100 \mathrm{~s} . \mathrm{m}^{-1}$ na área de pastagem; (ii) $\mathrm{r}_{\mathrm{s}}$ varia ao longo do dia e sazonalmente, com medianas passando de 40 no período da manhã, para $150 \mathrm{~s} . \mathrm{m}^{-1}$ no final da tarde, na estação chuvosa na floresta e 50 a160 s.m $\mathrm{m}^{-1}$ no pasto. Estas medianas aumentam na estação seca, com as $r_{s}$ florestais que variam de 50 a $500 \mathrm{s.m} \mathrm{m}^{-1}$ e $\mathrm{r}_{\mathrm{s}}$ na pastagem a partir de $140 \mathrm{~s} . \mathrm{m}^{-1}$ chegando a mais de $1800 \mathrm{~s} . \mathrm{m}^{-1}$ nas tardes secas; (iii) $\Omega$ varia de 0,5 a 0,8 durante a estação chuvosa, e cai para valores inferiores a 0,5 nas tardes durante a estação seca, o que indica que, apesar de uma forte influência do saldo de radiação na perda evaporativa, em grande parte os fluxos da evapotranspiração são acoplados ao controle biótico pelo fechamento dos estômatos na vegetação, especialmente no pasto e durante períodos de seca.

Palavras-chave: fator de desacoplamento, resistência aerodinâmica, resistência estomática.

\section{INTRODUCTION}

The Amazonian rainforest is one of the most important biomes of the planet. It is of great relevance to the global climate and biodiversity, and it is considered the largest tropical rain forest of the world and a major contributor to surface evapotranspiration (Choudhury et al., 1998), influencing the global hydrological cycle and affecting atmospheric global circulation associated with tropical convection.

Besides being important for maintaining the regional climate and ecosystem, the Amazon forest also has an important role in the different regional climates of Brazil. The Amazonian ecosystem can be considered a source of water for other regions, as a significant amount of water vapor that evapotranspirates in Amazonia is transported to Center-west, Southeast and Southern regions of Brazil by low-level jets (Zemp, 2014; Arraut et al., 2012).

Although the Amazon forest is recognized as an important component of the regional and global climate system, the spatial and temporal variability of its hydrological functions is not completely understood; therefore, evaluating the seasonal and spatial variations of the water fluxes in the tropics is important (Werth and Avissar, 2004). These variations mainly depend on the characteristics of the vegetation, on energy processes and on water availability (Hasler and Avissar, 2007). Understanding of vegetation and climate interaction is commonly achieved by field measurements and by weather- and climate models simulations. These models use variables such as wind, air and soil temperatures, air humidity and soil moisture to estimate evapotranspiration through aerodynamic conductance and stomatal conductance. Werth and Avissar (2004) presented a review of four approaches to estimate evapotranspiration in Amazonia: the first is the relationship with radiation, the second is water balance, the third is global climate and the last is data assimilation. They observed the seasonality of ET in two groups: one that follows the seasonal cycle of net radiation, and another that follows the seasonal cycle of precipitation. This division motivates the following research questions: what controls the seasonal variation of ET in Amazonia? Is it mainly related to the available radiative energy or does the vegetation significantly control its variability, through its surface conductance that is susceptible to the seasonality of soil moisture?

Souza Filho et al. (2005) presented in their study evidence that supported the idea that the radiation and maybe other atmospheric variables are the main mechanism of control of the seasonal variation of ET in the Amazonia, while vegetation control plays a secondary role in ET. They used meteorological data from Caxiuanã, in Northeast Amazonia, to calculate the aerodynamic and surface conductance and the decoupling factor proposed by Jarvis and McNaughton (1986) during dry and wet periods. During the wet period surface conductance is higher than during the dry period (Souza Filho et al., 2005). On the other hand,

Rev. Ambient. Água vol. 12 n. 2 Taubaté - Mar. / Apr. 2017 
VPD, aerodynamic conductance, and solar radiation are higher during the dry period. But, even while presenting a higher surface conductance during the wet season, the vegetation does not prevent the ET from being higher during the dry season. From this analysis, they concluded that the control of atmospheric conditions over ET is dominant, with a decoupling factor close to 1 in the wet and dry seasons.

Da Rocha et al. (2009) investigated the seasonal behavior of water vapor flux and sensible heat flux in 7 flux towers in Brazil. These tower sites included tropical humid and semideciduous forest, transitional forest, floodplain (with physiognomies of Cerrado), and Cerrado sensu stricto. The authors observed that the control of the seasonality of ET was different in each biome, where evaporative demand (mainly the net radiation) plays the most important role in the tropical humid forests and soil moisture variation plays the most important role in the Cerrado.

In a more complete evaluation of differences in control of evapotranspiration in wet forest and seasonally dry forests, Costa et al. (2010) found that the wet equatorial sites are mainly driven by environmental factors, while in seasonally dry forests, ET is also controlled by biotic factors. This poses the question of whether the wet equatorial sites will remain largely resilient to dry season water stress or may begin to present signs of larger biotic control of water loss by the vegetation in case of increasing frequency of extreme dry seasons as predicted by some climate models. Moreover, a large fraction of Amazonia is also under pressure of deforestation for pasture or agriculture, and it is interesting to investigate how a vegetation type like pasture grass compares to the pristine rainforest in terms of biotic control of evapotranspiration and experiences dry season water shortages.

In this context, the goal of this study was to analyze the diurnal and seasonal variations of the aerodynamic resistance, stomatal resistance and the decoupling factor of Jarvis and McNaughton (1986) for three sites in Amazonia: a primary forest in Central Amazonia and a primary forest and a pasture in Southwestern Amazonia. The data used in the present work are measurements of evapotranspiration and meteorological variables. In Central Amazonia, the data were collected in the Cuieiras Biological Reserve near Manaus, AM, while in Southwestern Amazonia, the data were collected in the Jaru Biological Reserve and at the cattle ranch, Fazenda Nossa Senhora (FNS), close to Ji-Paraná, RO.

\section{METHODS AND SITE DESCRIPTION}

\subsection{Site description and instruments}

This study is based on the data of three sites in Amazonia. The first is located at the Cuieiras Forest Reserve, about $50 \mathrm{~km}$ north of Manaus, Amazonas state, Brazil. The site is known as $\mathrm{K} 34\left(2^{\circ} 36^{\prime} 32^{\prime \prime} \mathrm{S}, 60^{\circ} 12^{\prime} 33^{\prime \prime} \mathrm{W}\right)$ and it is part of the LBA project, which is responsible for the maintenance and administration of the site. The second site is located at Jaru Biological Reserve (Rebio-Jaru), about 100 km north Ji-Parana, Rondônia state, Brazil (10 4' S; 61 $\left.{ }^{\circ} 56^{\prime} \mathrm{W}\right)$. The third site is a pasture located about $50 \mathrm{~km}$ northwest of Ji-Paraná, Rondônia state, Brazil. The site is a cattle ranch known as Fazenda Nossa Senhora Aparecida (FNS) (10 45' S, 62 $22^{\prime} \mathrm{W}$ ), deforested in the late 1970s. The second and third sites are also part of LBA project.

The K34 site is covered by primary forest, with diverse vegetation and a vast number of species. The leaf area index (LAI) of the area is about 5-6. The index is in accordance with that estimated by Hasler and Avissar (2007). The climate is classified as Af according to the Köpen classification (tropical rainforest climate), with temperatures varying from 19 to $39^{\circ} \mathrm{C}$. The rainfall regime is divided into two seasons: rainy (November to May) and dry (August to October). The annual average precipitation varies from 1800 to $2800 \mathrm{~mm}$ year ${ }^{-1}$. More information about the climate of the region is found in Araújo et al. (2002). 
Besides conventional meteorological and short and long wave radiation sensors, an eddy covariance system is installed at a 53-m high tower measuring the energy, water and carbon fluxes of the K34 site. The system is composed of a tridimensional sonic anemometer (Solent 1012R2, Gill Instruments, UK) and a fast-response infrared gas analyzer ("Infra-Red Gas Analyzer", IRGA) (LI-6262, Li-Cor, EUA). The data was recorded in 10.4 Hz sampling (Araujo et al., 2002).

The Rebio Jaru is a "terra firme" forest, with relatively stronger rainfall variability than $\mathrm{K} 34$, which varies annually from 1250 to $2500 \mathrm{~mm}$. The forest has a mean height of about $35 \mathrm{~m}$, but some of the higher trees reach up to $45 \mathrm{~m}$. The air temperature shows no clear seasonal pattern, ranging on average between 22 and $27^{\circ} \mathrm{C}$; however, a significant drop in humidity and a reduction in rainfall is observed during the dry seasons. The same measurements as in K34 were collected, but at the height of $62.7 \mathrm{~m}$ (Von Randow et al., 2004).

The FNS is covered by Brachiaria brizantha (A. Rich.) Stapf. grasses, with an average LAI of 2.0 (Zanchi et al., 2009). The climate of the region is equatorial, warm and moist, with temperature varying from 17 to $32^{\circ} \mathrm{C}$. The rainfall regime follows the same patterns as in Rebio Jaru. The measurements from FNS analyzed in this work are from an automatic weather station (AWS) installed in a $5.5 \mathrm{~m}$ tower, providing measurements of the most common meteorological variables. The tower counts with an eddy correlation system similar to the one installed at K34. Other details of the microclimatology of the region can be found in the work of Von Randow et al. (2004).

In Rondônia, the topography is generally flat and the soil is classified as medium-textured red-yellow podzol (Podzólico vermelho amarelo A moderado textura media-Brazilian Soil Taxonomy, typic paleudult—or orthic acrisol—FAO).

\subsection{Evapotranspiration}

In the evaporation process of a vegetated surface two resistances can be considered as controlling factors: the stomatal resistance $\left(r_{s}\right)$ and the aerodynamical resistance $\left(r_{a}\right)$. In a vegetated surface, the path of turbulent diffusion from the surface to the atmospheric boundary layer is frequently analyzed through these two resistances. The stomatal resistance is related to the physiology of the plant. It is the mechanism that better represents the control of the evapotranspiration in the plant. The transpiration in the leaves accounts for approximately $90 \%$ of the water loss of the plant (Lambers et al., 2008), which reflects the importance of assessing this resistance.

The stomatal resistance was calculated by inverting the Penman Monteith (Equation 1).

$$
r_{s}=\left[\frac{\left(\rho_{a} C_{p} V P D\right)}{\gamma L E}-r_{a}\left(1-\frac{\Delta H}{\gamma L E}\right)\right]
$$

where :

$\rho_{a}$ is the air density $\left(\mathrm{kg} \mathrm{m}^{-3}\right)$,

$C_{p}$ is the specific heat of air at constant pressure $\left(\mathrm{Jkg}^{-1}{ }^{\circ} \mathrm{C}^{-1}\right)$;

VPD is given in $\mathrm{hPa}$;

$\gamma$ is the psychometric constant $\left(\mathrm{hPa}^{\circ} \mathrm{C}^{-1}\right)$;

$\mathrm{LE}$ is the latent heat flux $\left(\mathrm{Wm}^{-2}\right)$;

$\Delta$ is the slope of the saturation vapor curve $\left(\mathrm{hPa}^{\circ} \mathrm{C}^{-1}\right)$; and

$\mathrm{H}$ is the sensible heat flux $\left(\mathrm{Wm}^{-2}\right)$. 
On the other hand, the aerodynamic resistance is closely related to the planetary boundary layer and to the turbulent atmospheric processes. The aerodynamic resistance can be defined as the parameter that represents the resistance to the turbulent activity in the layer of air close to the surface. This turbulent activity is responsible for the transport of sensible and latent heat and water fluxes.

The aerodynamic resistance was calculated by Equation 2, following Costa et al. (2010).

$$
r_{a}=\frac{\bar{u}}{u_{*}^{2}}
$$

where:

$\bar{u}$ is the above canopy mean horizontal wind speed and

$u_{*}^{2}$ is the friction velocity.

This formulation is simpler than an explicit account of stability effects on the turbulence aerodynamics, but we prefer to use it to avoid large possible errors introduced in the calculation of stability functions in Amazonia and to make it more comparable to previous works of Hasler and Avissar (2007) and Costa et al. (2010). The unity that commonly represents the aerodynamic and surface resistances is $\mathrm{s} \mathrm{m}^{-1}$.

A useful tool to characterize the processes of exchange between the canopy and atmosphere and the control of these processes considering their seasonality is the decoupling factor $(\Omega)$ proposed by Jarvis and McNaughton (1986) as in Equation 3.

$$
\Omega=\frac{1}{1+[\gamma /(\delta+\gamma)]\left(\mathrm{r}_{\mathrm{s}} / \mathrm{r}_{\mathrm{a}}\right)}
$$

The decoupling factor is an index that allows evaluating whether the evapotranspiration process is mainly controlled by the vegetation or is decoupled from the surface and mainly limited by the energy available: the closer to 1 the greater the control of the atmospheric conditions (mainly available energy), while a value close to 0 indicates a strong influence of vegetation (strong coupling between the evapotranspiration and biological activity of stomata opening or closing).

The analysis in this work includes the wet and dry periods of 2000. In the three sites, the period analyzed was 90 days, from January to March (wet season) and also 90 days from August to October (dry season). These periods were chosen to represent the seasonality of the sites; therefore, we chose the months that the vegetation is most likely subject to contrasting wet/dry stress conditions (middle of wet season versus end of dry season).

All data were recorded in half-hour intervals and the calculations of the eddy fluxes were performed using an in-house developed software written in FORTRAN, which can be adapted to a number of different hardware configurations and program options. The program was configured to apply two-axis rotations to align the coordinate frame with the mean streamlines and force the mean vertical component $(\mathrm{w})$ to zero and to perform standard frequency-response corrections. No detrending method was applied to the signals. Generally, for these sites and instrumental setup, apart from the coordinate rotations, the corrections are relatively small and do not represent large uncertainty factors in the final values (Kruijt et al., 2004).

Based on this information, the aerodynamical and stomatal resistances and the decoupling factors were calculated and only daytime periods (from 8 am to $6 \mathrm{pm}$ ) are presented, to focus the study on the analysis of time periods where the vegetation control of evapotranspiration would be greatest. 


\section{RESULTS AND DISCUSSION}

In Central Amazonia, the maximum monthly precipitation registered during the year 2000 was $458 \mathrm{~mm}$ in April and the minimum was $89 \mathrm{~mm}$ in August. The total precipitation of the year was about $2620 \mathrm{~mm}$. In Southwestern Amazonia, the total precipitation for the year was $1760 \mathrm{~mm}$, while the highest monthly precipitation was $274 \mathrm{~mm}$ in October (which already indicates the beginning of the following wet season), followed by $264 \mathrm{~mm}$ in January. The driest month was August, reaching $30 \mathrm{~mm}$.

Figure 1 presents the monthly values of precipitation minus reference evapotranspiration (P-ETo), as calculated using FAO standard recommendations (Allen et al., 1998) for the three sites. We observe that the sites in Southwestern Amazonia (Jaru and FNS) are subject to drier conditions during June through August, with significantly negative values of P-ETo.

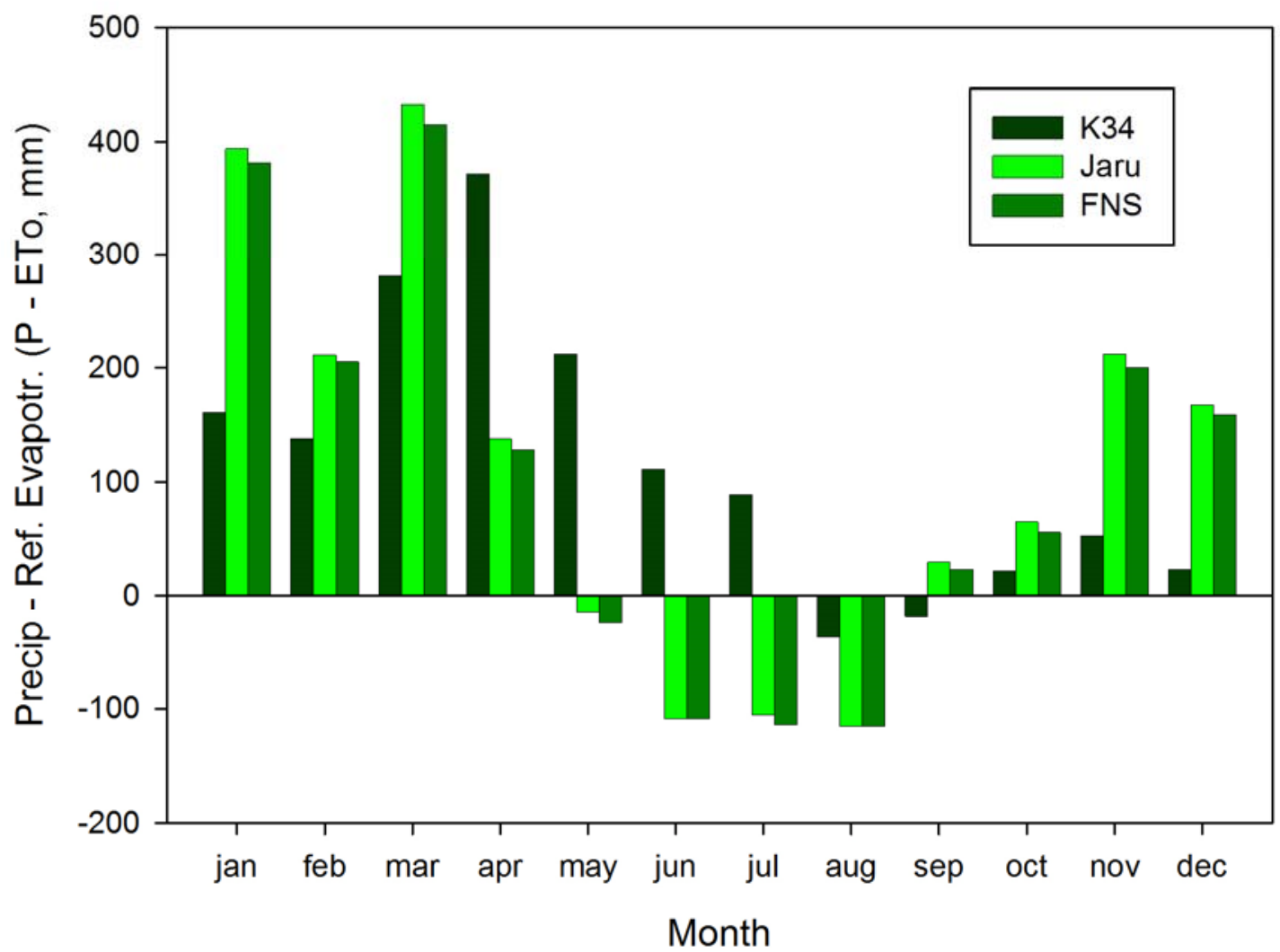

Figure 1. Monthly totals of the difference between precipitation and reference evapotranspiration (P - ETo), computed using FAO standard method.

Daily averages of the net radiation in K34 from January to March vary between 24 and $223 \mathrm{Wm}^{-2}$ (not shown). This variation is slightly lower in the period from August to October, when the net radiation varied from 59 to $207 \mathrm{Wm}^{-2}$. Corresponding values in Rebio Jaru vary from 30 to $243 \mathrm{Wm}^{-2}$ (wet period) and from 40 to $205 \mathrm{Wm}^{-2}$ (dry period). In the pasture site, the net radiation variation is lower when compared to the forests. The highest variation in daily average net radiation in the pasture also happened from January to March, but in a lower range: from 42 to $202 \mathrm{Wm}^{-2}$, and during the dry months (June to August), varied from 37 to $149 \mathrm{Wm}^{-2}$. 
Results of the measurements of vapor pressure deficit (VPD), aerodynamic resistance $\left(r_{a}\right)$, stomatal resistance $\left(r_{s}\right)$ and decoupling factor $(\Omega)$ during wet and dry season periods are presented in Figures 2 to 4 . Each point in the line graphs is the median of observed values at each half hour, and the bars present the interval between the first and third quartiles. In this way, the values presented within the bars include 50 percent of the observations at each time.

Figure 2 shows the diurnal median values for the K34 forest site, which is located in Central Amazonia (Amazonas state). Figures 3 and 4 show the same variables, but measured at the Jaru forest site and FNS pasture site, which are located in Southwest Amazonia (Rondônia state). In general, results from the two forest sites are similar, but some changes in the values are noted in the pasture vegetation.

First observing the measurements at the forest sites (Figures 2 and 3), clear effects of the dry season are noted in the measurements of VPD (Figures $2 \mathrm{a}$ and $3 \mathrm{a}$ ), where the maximum diurnal values reach $\sim 20 \mathrm{hPa}$ in both forests, compared to less than $10 \mathrm{hPa}$ during the wet season. The $r_{a}$, on the other hand, almost does not change from the wet to the dry season (Figures $2 \mathrm{~b}$ and $3 \mathrm{~b}$ ), remaining below $30 \mathrm{~s} / \mathrm{m}$ during daytime hours. This means that the turbulent activity and roughness characteristics change little from one season to the other at those forests.

The forest stomatal resistances also vary significantly between seasons (Figure $2 \mathrm{c}$ and $3 \mathrm{c}$ ). During the wet season, the daily variation is lower than during the dry season, ranging from around 30 to $150 \mathrm{~s} / \mathrm{m}$ in the wet season and from 50 to $500 \mathrm{~s} / \mathrm{m}$ in the dry season (with higher dispersion of values at Jaru, Figure 3c).

Figures $2 \mathrm{~d}$ and $3 \mathrm{~d}$ present the diurnal variation of $\Omega$ for both forests. During the wet season, $\Omega$ shows a decreasing trend throughout the day, ranging from 0.8 to 0.5 . During the dry season, $\Omega$ remains around 0.6 in the morning at K34 (Figure 2d), then drops to 0.4 in the afternoon. However, it drops significantly lower at Jaru throughout the day (Figure 3d). According to Jarvis and McNaughton (1986), this means that for both forests the evapotranspiration in the wet season is mainly controlled by the available energy, especially during morning hours, but trending to some influence of stomatal resistances during the day. During the dry season, the stomata play a stronger role in controlling forest transpiration, especially in the afternoon hours. Understanding mechanisms of how evapotranspiration is controlled in vegetated surfaces is of crucial importance, since the evapotranspiration processes connect the vegetation function and regional climate via their role in the partitioning of radiative energy into heat and moisture supply from the surface to the atmosphere.

The measurements at the pasture site are even more sensitive to the seasonal variations (Figure 4). Median VPD reaches $23 \mathrm{hPa}$ in the dry season (Figure 4a), while remaining below $11 \mathrm{hPa}$ in the wet season. Variations in $r_{a}$ are also more pronounced than in the forests (Figure $4 \mathrm{~b}$ ), but these seasonal changes are more complex to interpret, because they are largely sensitive to grazing by cattle, which alters the roughness of the grass vegetation not necessarily consistent with our selection of wet and dry periods. Still, it can be observed that in the pasture $r a$ is higher than in the forests in both seasons, as a result of higher roughness of the forest vegetation.

The seasonal variations in $r_{\mathrm{s}}$ at FNS are similar to those in the forest sites in the wet season, varying from 50 to $150 \mathrm{~s} / \mathrm{m}$, while in the dry season the variation is much higher, from 100 to $1000 \mathrm{~s} / \mathrm{m}(\sim 2000$ at $6 \mathrm{pm}$, Figure $4 \mathrm{c})$, pointing to more intense water stress during the dry season in the pasture vegetation.

The behavior of $\Omega$ at the pasture is also different than in the forests, although also qualitatively indicating the higher control of transpiration by stomatal resistance during the dry periods (Figure $4 \mathrm{~d}) . \Omega$ remains high $(\sim 0.8)$ during the whole day in the wet season and drops significantly (from 0.8 to 0.3 ) during the day in the dry season. 

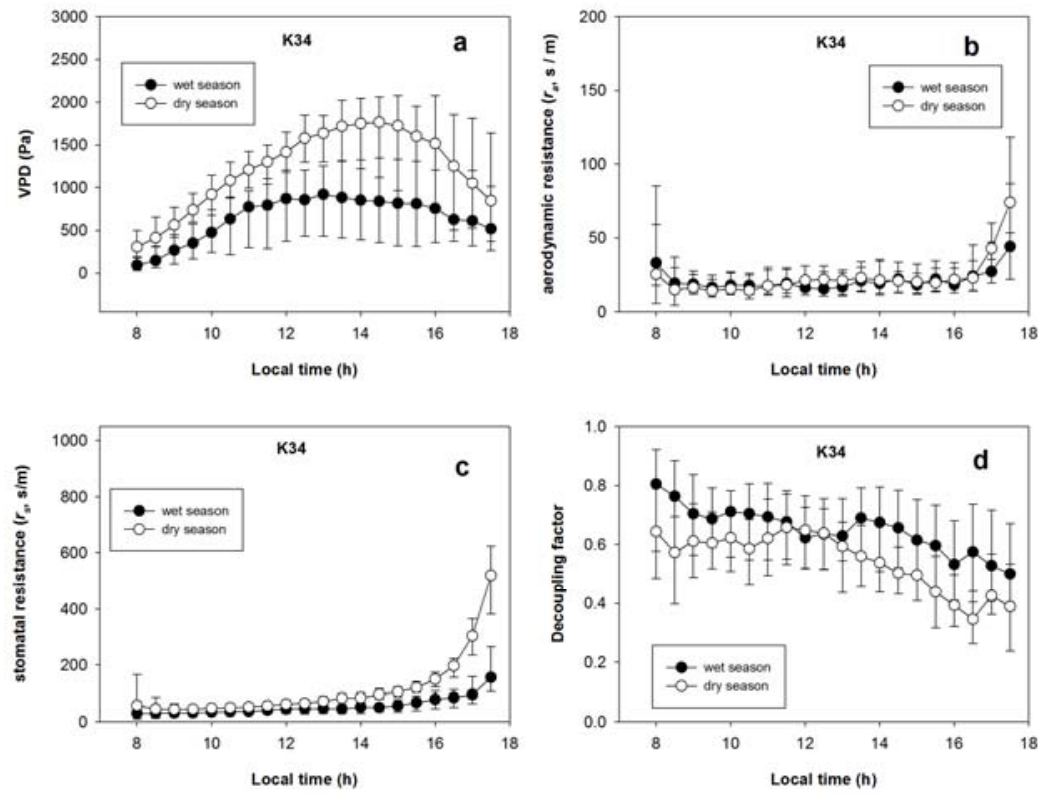

Figure 2. Distribution of hourly measurements of (a) vapor pressure deficit (VPD), (b) aerodynamic resistance, (c) stomatal resistance and (d) the decoupling factor, $\Omega$, during the daytime, observed in wet and dry periods of 2000 at K34 forest site. Symbols represent the median of observations in each hour and the vertical bars represent the interval between $1^{\text {st }}$ and $3^{\text {rd }}$ quartiles.
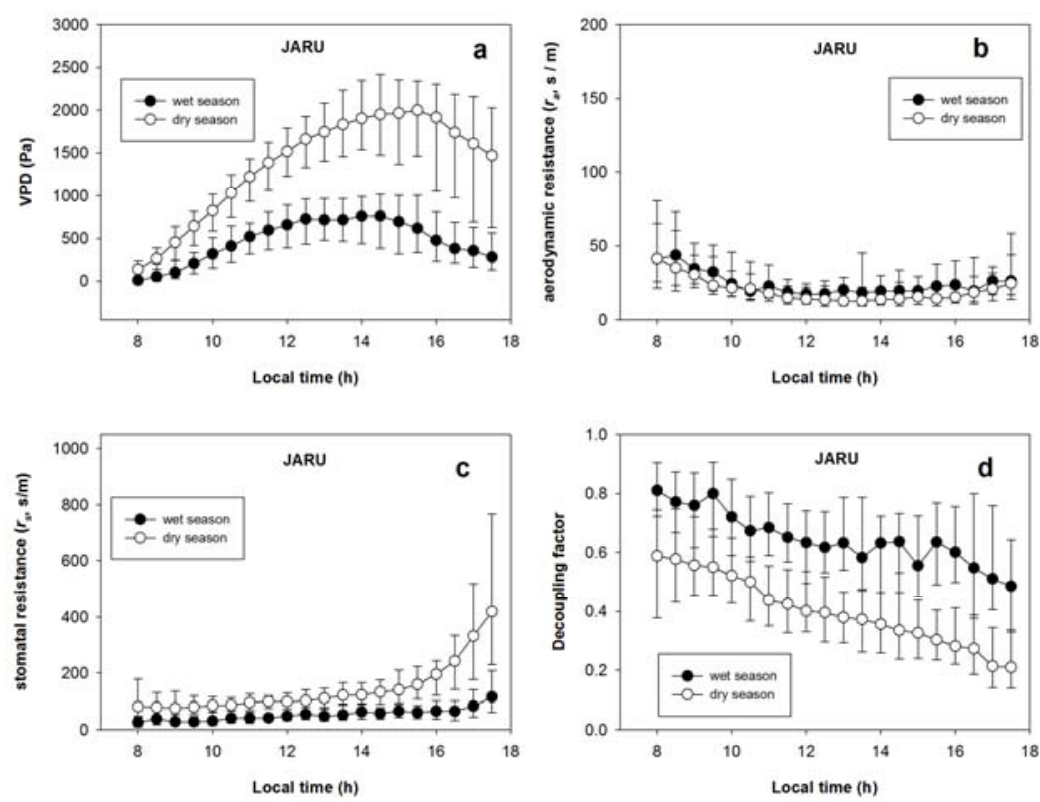

Figure 3. Distribution of hourly measurements of (a) vapor pressure deficit (VPD), (b) aerodynamic resistance, (c) stomatal resistance and (d) the decoupling factor, $\Omega$, during the daytime, observed in wet and dry periods of 2000 at Jaru forest site. Symbols represent the median of observations in each hour and the vertical bars represent the interval between 1 st and 3rd quartiles. 

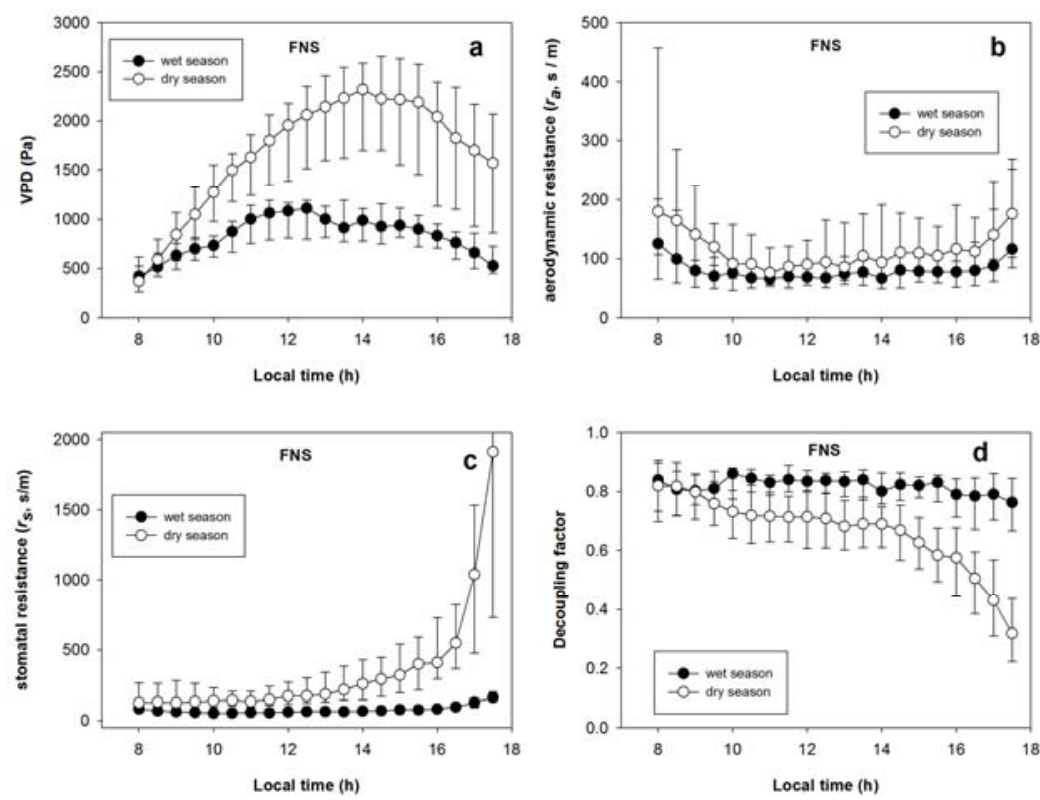

Figure 4. Distribution of hourly measurements of (a) vapor pressure deficit (VPD), (b) aerodynamic resistance, (c) stomatal resistance and (d) the decoupling factor, $\Omega$, during the daytime, observed in wet and dry periods of 2000 at the FNS pasture site. Symbols represent the median of observations in each hour and the vertical bars represent the interval between 1 st and 3rd quartiles.

For reference use in the climate models, in Table 1 we synthesize recommended values of the aerodynamic and stomatal resistances in the three sites. The $r_{a}$ values were estimated from an approximate average of the medians from 9:00 to 16:00 h, to avoid the hours of transition to nighttime. The $r_{s}$ reference values are presented as a range from lower resistances recommended for use in the morning time to higher resistances to represent stronger stomatal control during the afternoon.

Table 1. Reference values for aerodynamic and stomatal resistances, estimated in this study.

\begin{tabular}{lcc}
\hline & $r_{a}(\mathrm{~s} / \mathrm{m})$ & $r_{s}(\mathrm{~s} / \mathrm{m})$ \\
\hline Forest K34, wet season & 20 & $40-150$ \\
Forest K34, dry season & 20 & $50-500$ \\
Forest Jaru, wet season & 20 & $40-100$ \\
Forest Jaru, dry season & 20 & $90-400$ \\
Pasture (FNS), wet season & 70 & $50-160$ \\
Pasture (FNS), dry season & 100 & $140-1800$ \\
\hline
\end{tabular}

Previous studies in the Amazon region have identified that evapotranspiration is directly connected to available energy, but also to aerodynamic transport mechanisms and biological control of transpiration, and that these controls vary according to the vegetation type or depending on periods of seasonal water deficits (e.g. Souza Filho et al., 2005, Hasler and Avissar, 2007, Costa et al., 2010, Von Randow et al., 2012, Christoffersen et al., 2014). Hasler 
and Avissar (2007) analyzed the seasonality of evapotranspiration in 8 sites in Amazonia; three of them are the K34, Rebio Jaru and FNS sites. However, the authors used a preliminary version of the dataset, which was possibly subject to large uncertainties due to problems in data collection and incomplete quality assessment. We now update and extend the analyses to address in more detail how these mechanisms vary throughout the daytime and seasonally in the two contrasting vegetation types of rainforest and pasture in the same region, and in a rainforest in a wetter climate.

Results obtained in this study show that the biological control of transpiration (represented by the stomatal resistance) presents both diurnal and seasonal variability. Daily, a low resistance marks it in the morning, increasing throughout the day to reach high values in the evening. Since the stomatal resistance is related to the opening or closure of stomata, these results indicate that there is restriction of transpiration related to water deficit in the plants.

Analyzing the aerodynamic resistances, we also found that turbulence caused by the roughness of the surface can be an important secondary factor and almost invariable from the wet to dry seasons in the production of evapotranspiration in the sites, especially in the forest. This is indicated by the low aerodynamic resistance observed, or, in its turn, high aerodynamic conductance related to high roughness of the forest surface.

The results obtained from the analysis of the Jarvis and McNaughton (1986) decoupling factor $(\Omega)$ may bring further insight into our discussion. The parameter varies between 0 and 1 , with values close to 1 indicating that the conditions of evapotranspiration are decoupled from surface resistances (that is, evapotranspiration is mainly related to the amount of available energy), and values close to 0 indicating a strong coupling between evapotranspiration and the atmospheric turbulent conditions and biological activity (that is, evapotranspiration is controlled not only by energy, but also by vegetation and other atmospheric conditions). Our results show that $\Omega$ presents not only a diurnal variation, but also a seasonal variation for both forests. During the wet season, evapotranspiration is controlled by atmospheric conditions, especially during the morning, dropping to around 0.5 at the end of the afternoon in both forest sites. On the other hand, in the dry season, stomata is responsible for controlling the transpiration in both forests, mainly at the end of the afternoon, with $\Omega$ dropping from around 0.6 to less than 0.4. A strong drop occurs in Rebio Jaru, which is lower than 0.5 already at the end of the morning. This indicates that, although energy limitation is a strong influence, there is a significant contribution of the biotic factors to the variability of evapotranspiration. FNS, in turn, shows that its evapotranspiration is controlled by the atmospheric conditions in the wet season, as well as in the dry season. However, during the dry season, $\Omega$ presents a diurnal variation, dropping to lower than 0.5 at the end of the afternoon, showing once more the restriction of transpiration related to water deficit in the plants during this period.

The radiative energy provided by solar radiation is the main forcing of the biophysical processes of interaction between the biosphere and the atmosphere, acting as the primary driver of evapotranspiration in Amazonia. This has been observed before, especially in the more 'equatorial' sites of Amazonia, such as the K34 (e.g. Da Rocha et al., 2009, Costa et al., 2010). However, there is also indication that some species in equatorial sites are sensitive to water stress if the dry season is prolonged or in artificial rainfall exclusion experiments (Meir et al., 2009). Our results further highlight the strong sensitivity that a land use change to pasture vegetation would cause: the pasture site clearly shows a large increase in stomatal resistance and transpiration control by the vegetation during the dry season.

Da Rocha et al. (2009) and Costa et al. (2010) discussed in their results a general seasonal behavior in evapotranspiration in different sites in Amazonia, corroborating the main control of evapotranspiration by abiotic / environmental conditions of available energy. The climate conditions that produce the tropical forests are generally characterized by frequent and 
abundant rainfall, which is certainly the case of forest in Central Amazonia near Manaus, and this makes less likely the occurrence of severe water stress. Due to this and also due to the large soil depth that tree roots may reach in the region, it is expected that these forests will remain resilient to annual dry seasons in the near future. However, if the occurrence of anomalous dry years increases in frequency due to climate change or a combination of climate change and increasing pressures of land use change substantially affect the ecosystem, it is realistic to expect a significant degradation of the forest into a less resilient forest type.

\section{CONCLUSIONS}

Analyzing measurements of vapor pressure deficit, aerodynamic resistance, stomatal resistance and of the decoupling factor, we studied the diurnal and seasonal variability of control of evapotranspiration in contrasting sites in Amazonia: a pristine rain forest in Central Amazonia (K34 site), a pristine forest (Jaru) and a pasture (FNS) in Southwestern Amazonia. Seasonal variations in precipitation minus reference evapotranspiration totals are similar conditions in the sites, however the pasture presents slightly higher vapor pressure deficit (drier conditions) in the dry season, and, especially because of the shallower roots in the pasture vegetation, we observe that these conditions reflect a different seasonal behavior of the evapotranspiration in the pasture compared to the forest sites.

Aerodynamic resistance does not change significantly from wet to dry season in either forest site, but it is higher in the pasture compared to the forests due to its lower roughness, and it is sensitive to cattle grazing. Stomatal resistance, on the other hand, exhibits large changes both throughout the day and from the wet to the dry seasons at the three sites. Daily, stomatal resistance shows low values in the morning, increasing to reach its maximum in the afternoon and early evening, which highlights the effect of increasing stomatal control of evapotranspiration as the day progresses. This effect is even more pronounced in the dry season, which evidences the occurrence of water stress in the pasture vegetation. During the wet season, soil water conditions are likely much more favorable, and the stomatal resistance is similar in the two vegetation types.

Results obtained for the decoupling factor also corroborate conclusions from previous studies that evapotranspiration is primarily driven by the conditions of available energy in Amazonia (decoupled from surface conditions) in the wet season, but, still, a significant biological control of transpiration by plants is observed in the dry season, especially in the pasture cover and in the afternoon.

\section{REFERENCES}

ALLEN, R. G.; PEREIRA, L. S.; RAES, D.; SMITH, M. Crop evapotranspirationGuidelines for computing crop water requirements. Rome: FAO, 1998. (FAO Irrigation and drainage paper, 56).

ARAÚJO, A. C.; NOBRE, A. D.; KRUIJT B.; ELBERS, J. A.; DALLAROSA, R.; STEFANI $P$. et al. Comparative measurements of carbon dioxide fluxes from two nearby towers in a central Amazonian rainforest: The Manaus LBA site. Journal Geophysical Research: Atmospheres, n. 107, p. 58-1-58-20, 2002. http://dx.doi.org/10.1029/2001JD000676

ARRAUT, J. M.; NOBRE, C.; BARBOSA, H. M.; OBREGON, G.; MARENGO, J. Aerial rivers and lakes: looking at large-scale moisture transport and its relation to Amazonia and to subtropical rainfall in South America. Journal of Climate, v. 25, p. 543-556, 2012. http://dx.doi.org/10.1175/2011JCLI4189.1 
CHOUDHURY, B. J.; DIGIROLAMO,N. E.; SUSSKIND,J.; DARNELL,W. L.; GUPTA, S. K.; ASRAR, G. A biophysical process-based estimate of global land surface evaporation using satellite and ancillary data II. Regional and global patterns of seasonal and annual variations. Journal Hydrology, n. 205, p. 186-204, 1998.

http://dx.doi.org/10.1016/S0022-1694(97)00149-2

CHRISTOFFERSEN, B. O.; RESTREPO-COUPE, N.; ARAIN, M. A.; BAKER, I. T.; CESTARO, B. P.; CIAIS, P. et al. Mechanisms of water supply and vegetation demand govern the seasonality and magnitude of evapotranspiration in Amazonia and Cerrado. Agricultural and Forest Meteorology, n. 191, p. 33-50, 2014. http://dx.doi.org/10.1016/j.agrformet.2014.02.008

COSTA, M. H.; BIAJOLI, M. C.; SANCHES, L.; MALHADO, A. C. M.; HUTYRA, L. R.; DA ROCHA, H. R.; AGUIAR, R. G.; DE ARAÚJO, A. C. Atmospheric versus vegetation controls of Amazonian tropical rain forest evapotranspiration: Are the wet and seasonally dry rain forests any different? Journal Geophysical Research: Biogeosciences, n. 115, p. 1-9, 2010. http://dx.doi.org/10.1029/2009JG001179

DA ROCHA, H. R.; MANZI, A. O.; CABRAL, O. M.; MILLER, S. D.; GOULDEN, M. L.; SALESKA, S. R. et al. Patterns of water and heat flux across a biome gradient from tropical forest to savanna in Brazil. Journal of Geophysical Research: Biogeosciences, n. 114, p. 1-8, 2009. http://dx.doi.org/10.1029/2007JG000640

HASLER, N.; AVISSAR, R. What Controls Evapotranspiration in the Amazon Basin? Journal of Hydrometeorology, n. 8, p. 380-395, 2007. http://dx.doi.org/10.1175/JHM587.1

JARVIS, P. G.; MCNAUGHTON, K. G. Stomatal control of transpiration: Scaling Up from Leaf to Region. Advances in Ecological Research, n. 15, p. 1-49, 1986. http://dx.doi.org/10.1016/S0065-2504(08)60119-1

KRUIJT, B.; ELBERS, J. A.; VON RANDOW, C.; ARAUJO, A. C.; OLIVEIRA, P. J.; CULF, A. et al. The robustness of eddy correlation fluxes for Amazon rain forest conditions. Ecological Applications, n. 14, suppl. 4, p. S101-S113, 2004.

http://dx.doi.org/10.1890/02-6004

LAMBERS, H.; CHAPIN, F. S.; PONS, T. L. Plant physiological ecology. New York: Springer, 2008. $605 \mathrm{p}$.

MEIR, P.; BRANDO, P. M.; NEPSTAD, D.; VASCONCELOS, S.; COSTA, A. C. L.; DAVIDSON, E. et al. The effects of drought on Amazonian rain forests. In: KELLER, M.; BUSTAMANTE, M.; GASH, J.; SILVA DIAS, P. (Eds.). Amazonia and Global Change. Washington D.C: American Geophysical Union, 2009. p. 429-449. (Geophysical Monograph Series, v. 186).

SOUZA FILHO, J. D. C.; RIBEIRO, A.; COSTA, M. H.; COHEN, J. C. P. Mecanismos de controle da variação sazonal da transpiração de uma floresta tropical no nordeste da amazônia. Acta Amazônica, n. 35, p. 223-229, 2005. http://dx.doi.org/10.1590/S004459672005000200012

VON RANDOW, C.; MANZI A. O.; KRUIJT, B.; OLIVEIRA, P. J.; ZANCHI, F. B.; SILVA, L. et al. Comparative measurements and seasonal variations in energy and carbon exchange over forest and pasture in South West Amazonia. Theoretic al and Applied Climatology, n. 78, p. 5-26, 2004. http://dx.doi.org/10.1007/s00704-004-0041-z 
VON RANDOW, R. C. S.; VON RANDOW, C.; HUTJES, R. W. A.; TOMASELLA, J.; KRUIJT, B. Evapotranspiration of deforested areas in central and southwestern Amazonia. Theoretical and Applied Climatology, n. 109, p. 205-220, 2012. http://dx.doi.org/10.1007/s00704-011-0570-1

WERTH, D.; AVISSAR, R. The Regional Evapotranspiration of the Amazon. Journal of Hydrometeorology, n. 5, p. 100-109, 2004. http://dx.doi.org/10.1175/15257541(2004)005\%3C0100:TREOTA\%3E2.0.CO;2

ZANCHI, F. B.; WATERLOO, M. J.; AGUIAR, L. J. G.; VON RANDOW, C.; KRUIJT, B.; CARDOSO, F. L. et al. Estimativa do Índice de Área Foliar (IAF) e Biomassa em pastagem no estado de Rondônia, Brasil. Acta Amazônica, v. 39, n. 2, p. 335-348, 2009. http://dx.doi.org/10.1590/S0044-59672009000200012

ZEMP, D. C.; SCHLEUSSNER, C.-F.; BARBOSA, H. M. J.; VAN DER ENT, R. J.; DONGES, J. F.; HEINKEL, J. et al. On the importance of cascading moisture recycling in South America. Atmospheric Chemistry Physics, v. 14, p. 13337-13359, 2014. http://dx.doi.org/10.5194/acp-14-13337-2014 


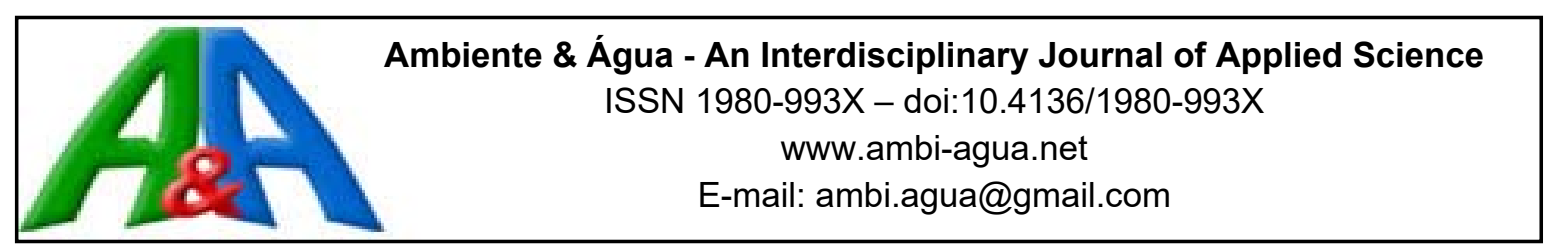

\title{
The use of caffeine as a chemical marker of domestic wastewater contamination in surface waters: seasonal and spatial variations in Teresópolis, Brazil
}

\author{
doi:10.4136/ambi-agua.1974
}

Received: 21 Jul. 2016; Accepted: 20 Dec. 2016

\author{
Eline Simões Gonçalves ${ }^{1 *}$; Silvana Vianna Rodrigues²; \\ Emmanoel Vieira da Silva-Filho ${ }^{3}$ \\ ${ }^{1}$ Fundação Oswaldo Cruz (FIOCRUZ), Rio de Janeiro, RJ, Brasil \\ Escola Nacional de Saúde Pública (ENSP) \\ ${ }^{2}$ Universidade Federal Fluminense (UFF), Niterói, RJ, Brasil \\ Departamento de Química Analítica \\ ${ }^{3}$ Universidade Federal Fluminense (UFF), Niterói, RJ, Brasil \\ Programa de Pós-Graduação em Geociências / Geoquímica Ambiental \\ *Autor correspondente: e-mail: elinesg@gmail.com, \\ silvana@vm.uff.br, emmanoelvieirasilvafilho@id.uff.br
}

\begin{abstract}
This study assessed the suitability of caffeine as an indicator for surface water pollution by domestic wastewaters. Caffeine concentrations determined in samples collected in the Paquequer River, located at the city of Teresópolis, a medium-sized city in the State of Rio de Janeiro, Brazil, were compared to other conventional water quality parameters such as phosphate, nitrate and ammonium concentrations to determine whether it as an efficient indicator of anthropic pollution of an urban aquatic environment. The Paquequer River originates in a pristine area called Parque Nacional da Serra dos Órgãos (PARNASO) and it crosses the urban area of the city of Teresópolis. Water samples were collected at seven points along the river, considering the periods of rain (summer) and drought (winter) and the different uses of land. Caffeine was measured using Solid Phase Extraction (SPE) and High Performance Liquid Chromatography (HPLC). Caffeine concentrations were found in a range from 0.16 to $47.5 \mu \mathrm{g} \mathrm{L}^{-1}$. The results show a positive relation between caffeine, phosphate, nitrate and ammonium concentrations and the increase of wastewater load, suggesting that caffeine can be used as an indicator of surface water pollution by domestic wastewaters.
\end{abstract}

Keywords: Anthropic contamination, caffeine, surface waters.

\section{O uso da cafeína como marcador químico de contaminação por esgoto doméstico em águas superficiais: variações sazonais e espaciais em Teresópolis, Brasil}

\section{RESUMO}

O uso da cafeína como um indicador para a poluição das águas superficiais por esgoto doméstico foi avaliado neste estudo. Concentrações de cafeína foram determinadas em amostras coletadas no rio Paquequer, localizado em Teresópolis, uma cidade de médio porte do Estado do Rio de Janeiro, Brasil, e foram comparadas com outros parâmetros convencionais de 
qualidade da água (fosfato, nitrato e amônio), de modo a caracterizá-la como um indicador eficaz da poluição antrópica do ambiente aquático urbano. O rio Paquequer nasce em uma área de preservação ambiental, o Parque Nacional da Serra dos Órgãos (PARNASO) e atravessa a área urbana da cidade de Teresópolis. Amostras foram coletadas em sete pontos ao longo do rio, considerando-se os períodos de chuva (verão) e seca (inverno) e os diferentes usos da terra. A cafeína foi determinada utilizando as técnicas de extração por fase sólida (SPE) e cromatografia líquida de alta eficiência (HPLC). As concentrações de cafeína foram encontradas numa faixa de $0,16-47,5 \mathrm{mg} \mathrm{L}^{-1}$. Os resultados mostram uma relação positiva entre as concentrações de cafeína, fosfato, nitrato e amônia, e o aumento da carga de esgoto, o que sugere que a cafeína pode ser utilizada como um possível indicador de contaminação por esgoto doméstico em águas superficiais.

Palavras-chave: Águas superficiais, cafeína, contaminação antrópica.

\section{INTRODUCTION}

From rivers to the coastal zone, continental aquatic systems are resources progressively used and transformed by humans via social and economic activity as a result of population growth, education, urbanization, industrialization, water engineering, and national and international environmental regulations (Meybeck, 2003). All of these factors can contribute to water pollution, particularly those related to population growth and inadequate disposal of sewage in water systems.

During the last decades, an increasing number of organic compounds have been detected in surface waters, generating great concern about pollution of this essential natural resource. Contaminated aquatic ecosystems can be a serious threat to human health and also to the environment. Some examples of water contamination on environmental health are pathogenic outbreaks such as diarrhea, the collapse of fisheries, decreased biodiversity, etc. (Myers et al., 2013). Consequently, protection of aquatic ecosystems is a major priority for humanity.

Domestic, industrial and agricultural sewage disposal into water streams are important causes of water pollution. In this context, the identification of the origin of pollution will play a key role in determining appropriate remedial measures. Such identification is accomplished through the use of a feasible marker. The ideal marker is one that is easily evaluated and that allows the unambiguous determination of the source and magnitude of contamination.

According to Bradley et al. (2007), an indicator applied to evaluate environmental contamination caused by humans ideally should be widely used, behave in the environment similarly to other major contaminants derived from domestic effluents, be present in relevant concentrations and detectable using available analytical methods and have an incomplete removal at wastewater treatment plants.

Traditionally, monitoring and tracking infiltrations of domestic wastewater in treated waters could be done by microbiological or chemical analyses. Microbiological analysis is conducted to investigate the presence of bacterial markers (like Escherichia coli), but there are disadvantages such as time-consuming analyses, limited source specificity (from animal, human or natural occurrences) and relatively short survival times in natural waters (Burge et al., 2003; Glassmeyer et al., 2005). Chemical determination is usually focused on nitrogen compounds such as ammonium, nitrate and nitrite. However, such analyses do not allow the identification of the origin of the pollution source (Sankararamakrishnan and Guo, 2005).

Owing to the recent development of analytical methods, several substances have been proposed as indicators of human contamination in urban water systems, such as caffeine, carbamazepine and sucralose. Caffeine is usually found in relatively high concentrations in 
waters streams and is easily measured. It has therefore been proposed as a potential wastewater tracer (Daneshvar et al., 2012; Spence, 2015).

The use of caffeine as indicator of sewage contamination is a relatively new analytical tool, especially when other indicators cannot reflect the source and magnitude of the observed contamination. Caffeine has a half-life of 30 days in natural environments, allowing its measurement in environmental samples even some time after initial contamination. Its behavior is more stable and conservative than that presented by others indicators, such as nitrogen, favoring the use of caffeine as a contamination indicator for domestic wastewater into surface waters.

Caffeine, (1,3,7-trimethylxanthine) is an alkaloid that occurs in more than 60 plant species and is ranked the number one drug worldwide, usually employed as a stimulant commonly found in coffee, tea, cacao, soft drinks and chocolate. It is also a component in hundreds of prescription and over-the-counter drugs, ranging from analgesics, to cold medicines, as a diuretic and as cardiac, cerebral and respiratory stimulant, and in "energy" dietary supplements (Burge et al., 2003; Gardinali and Zhao, 2002). Caffeine sources have claimed to improve endurance when included in athlete's diet and to increase the basal metabolic rate and caffeine is therefore classified by the International Olympic Committee (IOC) as a drug of abuse when it is present in human urine with concentrations higher than $12 \mu \mathrm{g} \mathrm{mL}^{-1}$ (Pesta et al., 2013).

The global average consumption of caffeine is about $70 \mathrm{mg}$ person $^{-1}$ day $^{-1}$ but varies in different countries (Chen et al., 2002). In Brazil, the average consumption is $171 \mathrm{mg} \mathrm{person}^{-1}$ day $^{-1}$, being coffee the main source of caffeine in the Brazilian diet (Camargo et al., 1999). Despite the fact that caffeine is extensively metabolized by humans with only approximately 3\% excreted unchanged in the urine (Tang-Liu et al., 1983), it is broadly found in the environment due to its continual introduction into the sewage system by the disposal of unconsumed coffee, tea or soft drinks down drains, and the rinsing of coffee pots and cups.

Caffeine is most likely to persist in the water column largely because of its high solubility $\left(13.5 \mathrm{~g} \mathrm{~L}^{-1}\right)$, low octanol-water partition coefficient $(\log \mathrm{Kow}=0.01)$ and negligible volatility. Additionally, caffeine is regularly and constantly consumed and discharged in sufficient quantities in wastewaters which allows easy analytical quantification. Its unequivocal anthropogenic origin with no potential biogenic sources make this substance a marker of choice since it complies with all prerequisites for a good tracer or anthropogenic marker of domestic contamination in natural waters (Daneshvar et al., 2012).

The environmental concentrations of caffeine range from "traces" to relatively high concentrations in influent wastewaters [up to $192 \mu \mathrm{g} \mathrm{L}-1$ (Gomez et al., 2007)]. In fact, several studies have reported the presence of caffeine in surface and ground waters across the world. It is common to find caffeine in effluent wastewater [0.07-126 $\mu \mathrm{g} \mathrm{L}^{-1}$ (Weigel et al., 2004)], in well water [1.7 $\mu \mathrm{g} \mathrm{L}^{-1}$ (Swartz et al., 2006)], in groundwater [0.23 $\mu \mathrm{g} \mathrm{L}^{-1}$ (Seiler et al., 1999)], in coastal waters [15-185 ng L-1 (Cantwell et al., 2016)] and surface water (112-781 ng L-1 [Daneshvar et al., 2012]). These studies show that caffeine can be easily found in aquatic environments.

In general, the determination of caffeine in environmental samples is taken concurrently with other target compounds by chromatographic multi-residue methods. In the majority of cases a solid-phase extraction step is applied in conjunction with High Pressure Liquid Chromatography (HPLC) and Gas Chromatography (GC) in order to allow detection of trace levels of the analytes in the samples. Chromatographic techniques (liquid or gas chromatography) using UV-Vis detector (as diode array detector - DAD) or mass spectrometry detector (MS) are the most used strategies (Cahill et al., 2004; Gardinali and Zhao, 2002; Ferreira, 2005; Verenitch et al., 2006). 
Teresópolis (Rio de Janeiro) was chosen to evaluate the applicability of caffeine as marker of water contamination caused by human activities, through the comparison between caffeine and other usual parameters of wastewater along the Paquequer River.

\section{MATERIALS AND METHODS}

\subsection{Study area and sampling sites}

The studied area was the Paquequer River located in the city of Teresópolis, a mountainous region of the State of Rio de Janeiro ( $870 \mathrm{~m}$ above the sea level), situated in the southeast region of Brazil. It has a drainage catchment of $269 \mathrm{~km}^{2}$ and its headwater is located in the Serra dos Órgãos National Park - PARNASO (22 $\left.26^{\prime} 58^{\prime \prime} \mathrm{S}, 42^{\circ} 59^{\prime} 08^{\prime \prime} \mathrm{W}\right)$ in a well-preserved Atlantic rain forest. The climate is super-humid tropical, with an annual average temperature of $18{ }^{\circ} \mathrm{C}$ and an annual precipitation between 1500 and $2500 \mathrm{~mm}$.

In its course, the Paquequer River crosses the urban area of the city of Teresópolis, with a population of approximately 125,000 inhabitants. The city of Teresópolis has no wastewater treatment plant, and so there are two ways to dispose of sewage: $35 \%$ of households use septic tanks or drains while in other households sewage is directly discharged into rivers through the rainwater drainage system $(\mathrm{CMN}, 2000)$. The Paquequer is the main water body receiving the majority of the urban runoff and sanitary effluents drained without any treatment from Teresópolis. Those characteristics make the basin of the Paquequer River an interesting area to evaluate the effectiveness of caffeine as an indicator of contamination by wastewater.

To conduct the study, seven sampling sites were chosen in the Paquequer River Basin, three located inside of a conservation area (one of them at the Beija-Flor Creek, a tributary of Paquequer River) and four other sites along the urban area of Teresópolis City (Figure 1). Water samples were collected on February $6^{\text {th }}$ (summer), March $27^{\text {th }}$ (beginning of spring) and October $4^{\text {th }}$ (end of winter) of 2007.

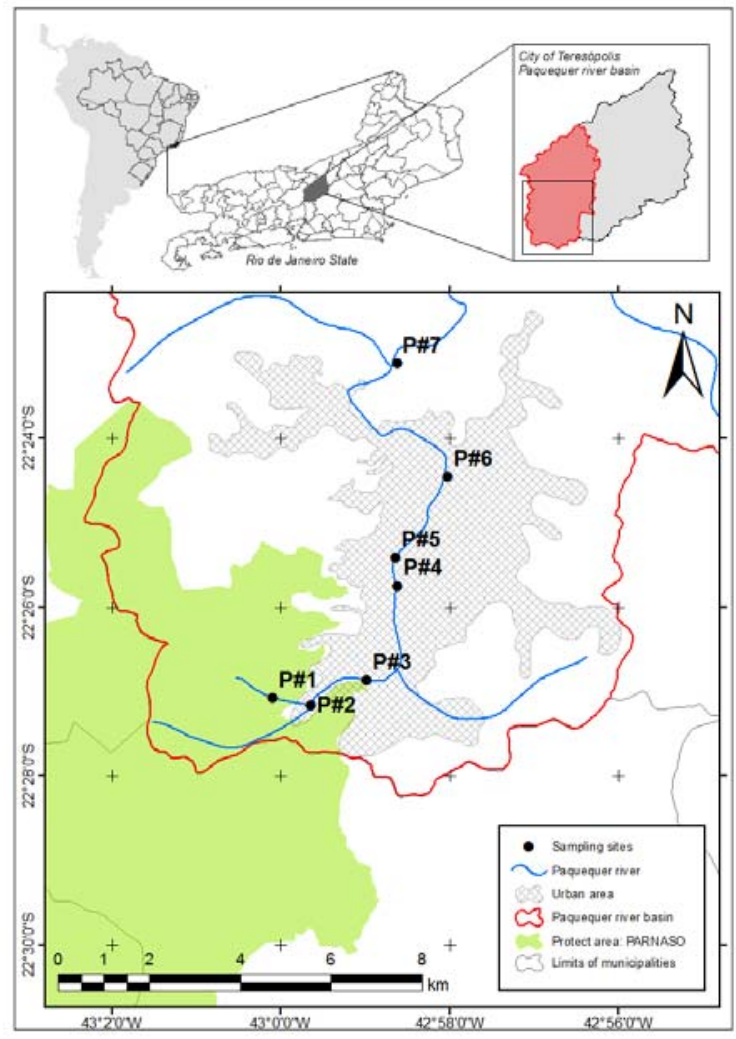

Figure 1. Location of the city of Teresópolis and the Paquequer River Basin. 


\subsection{Methodology}

\subsubsection{Sampling}

All samples for caffeine analysis were collected in duplicate in $1 \mathrm{~L}$ pre-cleaned amber glass bottles by submerging the sampling flasks approximately 10 centimetres below surface in the upstream direction. The samples were placed on ice, returned to the laboratory and stored in the dark at $<4^{\circ} \mathrm{C}$ until analysis, when they were not immediately filtered and extracted after arriving at the laboratory. Aliquots of filtered water samples were frozen for further analysis of nutrients.

The $\mathrm{pH}$ and conductivity were measured in situ using a calibrated portable conductivity meter (WTW) and a pH meter (Alfa Tecnoquímica), respectively.

\subsubsection{Nutrients analysis}

Nitrite, nitrate, ammonium, and phosphate were measured according to the Standard Methods (APHA et al., 2013). Ammonium was analysed by the indophenol's blue method. Nitrate was first reduced in a copper cadmium column and then analysed as nitrite. Nitrite was quantified by a reaction with sulfanilamide and $\mathrm{N}$-naphthyl ethylenediamine in an acid medium to form a diazo dye. Phosphate was measured by forming a complex with molybdate in an acid medium, reducing it with ascorbic acid. The absorbance of all samples was measured in a spectrophotometer at proper wavelength.

\subsubsection{Caffeine analysis}

The samples were filtered through a glass fiber filter with $0.7 \mu \mathrm{m}$ of porosity $(\mathrm{GF} / \mathrm{F}$, Whatman) to remove suspended matter and passed through a solid-phase extraction cartridge (SPE), using commercial Strata- $X^{\circledR}(500 \mathrm{mg}, 6 \mathrm{~mL}$ ) from Phenomenex (Torrence, CA), with the aid of a vacuum system at a flow rate of $3 \mathrm{~mL} \mathrm{~min}^{-1}$ (adapted from Burge et al., 2003).

Briefly, the cartridges were preconditioned with $6 \mathrm{~mL}$ of methanol and $6 \mathrm{~mL}$ of ultra-pure water (Millipore system). After the loading of samples, cartridges were dried under nitrogen for 1 hour and caffeine was recovered with $8 \mathrm{~mL}$ of methanol. The extracts were evaporated to dryness using a gentle stream of nitrogen. After reconstituting the samples to $500 \mu \mathrm{L}$ methanol:water $(30: 70, \mathrm{v} / \mathrm{v})$, analysis was performed. Procedural blanks were run periodically to check for caffeine contamination during extraction.

The determination of caffeine in the final extracts was carried out by High Pressure Liquid Chromatography (Bischoff Analysentechnik, Model 220) with detection by ultra-violet using a diode array detector (Shimadzu, model SPE-M104 VP) performed at $270 \mathrm{~nm}$. Chromatographic separation was accomplished using a Zorbax Eclipse ${ }^{\circledR}$ (Agilent, Model XDB C8, 4.6 x $150 \mathrm{~mm}$ I.D., $5 \mu \mathrm{m}$ ) column. The mobile phase was programmed to run isocratically with methanol: water $(30: 70, \mathrm{v} / \mathrm{v})$ at a flow-rate of $1 \mathrm{~mL} \mathrm{~min}^{-1}$.

Quantitative analyses were performed using external standards and the calibration graph was prepared for caffeine concentrations of $1.0 ; 2.5 ; 5,10.0 ; 20.0$ and $50.0 \mathrm{mg} \mathrm{L}^{-1}$. All standard solutions were prepared in methanol: water $(30: 70, \mathrm{v} / \mathrm{v})$.

The method detection limit (MDL) and the method quantification limit (MQL) for caffeine were determined by USEPA method EPA-821-R-03-005. Seven replicates of 1 L solution spiked with $0.15 \mu \mathrm{g} \mathrm{\textrm {L } ^ { - 1 }}$ caffeine were prepared and processed according to the same methodology applied to the real samples. The MDL was calculated by multiplying the standard deviation of the replicate analyses by the Student's $T$-value for 6 degrees of freedom at the $99 \%$ confidence level. The MQL was calculated by multiplying the standard deviation obtained in MDL calculations by 9 .

To evaluate the method recovery, simulated samples were prepared with ultrapure water and fortified with different concentrations of caffeine to final concentrations of $1,2.5,5,10,20$ and $50 \mu \mathrm{g} \mathrm{L}^{-1}$ (in duplicate to each concentration). These samples were processed according to

Rev. Ambient. Água vol. 12 n. 2 Taubaté - Mar. / Apr. 2017 
the same methodology applied to the real samples. The recoveries were calculated using the percentage formula: $(\% \mathrm{Rec})=[$ (observed concentration - blank concentration $) /$ (theoretical concentration)] $\mathrm{x} 100$.

\section{RESULTS AND DISCUSSION}

\subsection{Caffeine method performance}

Calibration curves were linear in the range of 0.1 to $50.0 \mathrm{mg} \mathrm{L}^{-1}$, with determination coefficients $\left(\mathrm{R}^{2}\right)$ higher than 0.999 . Recoveries ranged from $74.3 \%$ to $95.3 \%$, with a mean value of $85.7 \%( \pm 7.7)$. MDL and MQL, calculated at $0.15 \mu \mathrm{g} \mathrm{L}^{-1}$ standard solution was 0.05 and $0.15 \mu \mathrm{g} \mathrm{L}^{-1}$, respectively.

\subsection{Results overview}

The lowest caffeine concentrations were found in the samples collected inside the National Park (PARNASO) and the highest concentration was found along the urban area. Identical behavior was observed in the other parameters chosen to evaluate the water quality along the Paquequer River $\left(\mathrm{NO}_{3}{ }^{-}, \mathrm{NH}_{4}{ }^{+}, \mathrm{PO}_{4}{ }^{-3}\right.$ concentrations, $\mathrm{pH}$ and conductivity). In all samples, nitrite concentration was insignificant when compared to nitrate concentration. The results are presented in Table 1.

According to these results, caffeine concentrations in the basin of the Paquequer River ranged from $<0.15$ to $47.5 \mu \mathrm{g} \mathrm{L}^{-1}$ and during the studied period was (significantly) higher in Site \#6, the most anthropogenically impacted area among the seven sampling locations studied. This value is in agreement with other studies in some Brazilian rivers. Ferreira (2005), studying rivers of the drainage basin of Leopoldina (RJ), reported values from 60 to $357 \mu \mathrm{g} \mathrm{L}^{-1}$. Montagner and Jardim (2011) found caffeine concentrations from 0.2 to $73.9 \mu \mathrm{g} \mathrm{L}^{-1}$ in the Atibaia River, located in the region of Campinas (SP).

Table 1. Results found for water quality parameters in Paquequer River (Teresópolis/Brazil).

\begin{tabular}{|c|c|c|c|c|c|c|c|c|}
\hline Sites & & Period & $\begin{array}{c}\text { Caffeine } \\
\left(\mu \mathrm{g} \mathrm{L}^{-1}\right)\end{array}$ & $\begin{array}{c}\mathrm{NH}_{4}^{+} \\
\left(\mu \mathrm{mol} \mathrm{L}^{-1}\right)\end{array}$ & $\begin{array}{c}\mathrm{PO}_{4}^{-3} \\
\left(\mu \mathrm{mol} \mathrm{L}^{-1}\right)\end{array}$ & $\begin{array}{c}\mathrm{NO}_{3}^{-} \\
\left.(\mu \mathrm{mol} \mathrm{L})^{-1}\right)\end{array}$ & $\begin{array}{c}\text { Cond } \\
\left(\mu \mathrm{S} \mathrm{cm}^{-1}\right)\end{array}$ & $\mathrm{pH}$ \\
\hline \multirow{8}{*}{ 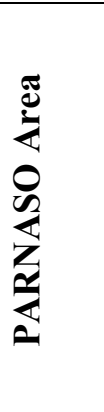 } & \multirow[t]{2}{*}{$\# 1$} & March $27^{\text {th }}$ & $<0.15$ & 8.52 & 0.06 & 0.62 & 9 & 6.62 \\
\hline & & Oct. $4^{\text {th }}$ & $<0.05$ & 4.80 & 0.26 & 0.91 & 11 & 7.35 \\
\hline & \multirow{3}{*}{$\# 2$} & Feb. $6^{\text {th }}$ & 0.21 & 12.08 & 0.15 & 12.20 & 5 & 6.65 \\
\hline & & $\operatorname{March} 27^{\text {th }}$ & 0.16 & 4.23 & 0.26 & 0.55 & 6 & 6.21 \\
\hline & & Oct. $4^{\text {th }}$ & $<0.15$ & 8.52 & 0.06 & 0.62 & 7 & 7.24 \\
\hline & \multirow{3}{*}{$\# \mathbf{3}$} & Feb. $6^{\text {th }}$ & 0.25 & 12.17 & 0.12 & 11.30 & 21 & 8.70 \\
\hline & & $\operatorname{March} 27^{\text {th }}$ & 0.17 & 7.86 & 0.10 & 8.60 & 17 & 7.86 \\
\hline & & Oct. $4^{\text {th }}$ & 0.17 & 4.80 & 0.31 & 8.10 & 18 & 7.70 \\
\hline \multirow{10}{*}{ 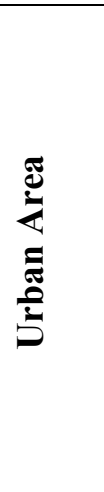 } & \multirow{2}{*}{$\# 4$} & March $27^{\text {th }}$ & 3.90 & 62.74 & 1.48 & 34.10 & 49 & 6.58 \\
\hline & & Oct. $4^{\text {th }}$ & 10.80 & 212.15 & 5.24 & 738.80 & 51 & 7.10 \\
\hline & \multirow{3}{*}{$\# 5$} & Feb. $6^{\text {th }}$ & 5.30 & 45.50 & 1.39 & 52.10 & 60 & 8.45 \\
\hline & & $\operatorname{March} 27^{\text {th }}$ & 5.50 & 8.62 & 3.26 & 44.00 & 83 & 7.10 \\
\hline & & Oct. $4^{\text {th }}$ & 7.97 & 260.63 & 6.99 & 610.60 & 60 & 6.86 \\
\hline & \multirow{3}{*}{$\# 6$} & Feb. $6^{\text {th }}$ & 12.50 & 97.41 & 2.97 & 187.50 & 76 & 7.57 \\
\hline & & March $27^{\text {th }}$ & 22.50 & 277.56 & 9.65 & 31.60 & 114 & 6.92 \\
\hline & & Oct. $4^{\text {th }}$ & 47.50 & 567.70 & 17.46 & 2.77 & 105 & 6.74 \\
\hline & \multirow{2}{*}{$\# 7$} & $\operatorname{March} 27^{\text {th }}$ & 6.20 & 172.98 & 3.76 & 28.50 & 85 & 6.91 \\
\hline & & Oct. $4^{\text {th }}$ & 15.40 & 359.40 & 12.22 & 4.26 & 85 & 6.40 \\
\hline
\end{tabular}




\subsubsection{Protected Area: PARNASO Area}

At sample points $\mathrm{P} \# 1, \mathrm{P} \# 2, \mathrm{P} \# 3$, located inside the PARNASO, the concentration of caffeine was expected to be virtually zero or very close to the limit of quantification. In fact, this was observed during the dry season (winter) but not during the rainy season (summer) when higher concentrations were found. However, comparing the caffeine concentration found at PARNASO with the concentration of ammonia and phosphate measured at the same points, no significant correlation at $95 \%$ confidence (Pearson coefficient 0.78 and -0.63 for ammonia and phosphate, respectively for $\mathrm{n}=6$ ) was found, suggesting the existence of other caffeine sources different than the domestic waste at this region. The presence of caffeine in the local vegetation could be one reason for these results. In this case, during the rainy season caffeine could be transported from plants and contaminated soils to the streams. Similarly, Peeler et al. (2006) found caffeine at concentration of $14 \mathrm{ng} \mathrm{L}^{-1}$ in the samples of its background area and explained this result as originating from plants containing this alkaloid. Another possibility is air circulation. As Teresópolis is located close to the coast but behind high mountains where PARNASO is situated, atmospheric circulation could bring to PARNASO caffeinecontaminated air originating from roasting factories located in the city, contaminating the environment and the clouds and consequently the rain.

\subsubsection{Urban area}

Along the urban area of Teresópolis, the water quality of the Paquequer River suffers intense degradation, due mainly to the inadequate discharge of wastewater. It was therefore expected that caffeine, ammonia, phosphate and nitrate concentrations would increase along the river. This was observed for caffeine concentrations, ammonia and phosphate (points $\mathrm{P} \# 4$, $\mathrm{P} \# 5$, $\mathrm{P} \# 6$ ). However, at $\mathrm{P} \# 7$ these concentrations were lower than those found at $\mathrm{P} \# 6$. The reason for this concentration decrease is dilution, since between points $\mathrm{P} \# 6$ and $\mathrm{P} \# 7$ the river crosses a region with lower urban density and is joined by some smaller water bodies.

At the points $\mathrm{P} \# 4, \mathrm{P} \# 5, \mathrm{P} \# 6$ and $\mathrm{P} \# 7$, significant correlations (Pearson, $\mathrm{n}=10,95 \%$ confidence) between caffeine and both ammonia and phosphate ( $r=0.87$ and 0.89 , respectively) were observed. The strong correlations found between these parameters support the hypothesis that they originated from sewage discharge. No correlation between caffeine and nitrate was found in the samples collected at the urban area $(r=-0.22)$. Also, the correlations between nitrate concentration and all other parameters were not significant.

The nitrate concentrations do not show a continuous increase in the samples along the urban area. Higher concentrations at points $\mathrm{P} \# 6$ and P\#7 were expected, but they showed a significant decrease compared with points $\mathrm{P} \# 4$ and $\mathrm{P} \#$ 5. In campaign 3 , this reduction was even more pronounced than in campaign 2, where the values found in $\mathrm{P} \# 6$ and $\mathrm{P} \# 7$ are around 200 times smaller than in $\mathrm{P} \# 4$ and $\mathrm{P} \# 5$. These results demonstrate that the nitrate concentrations in the river Paquequer varied independently of the increase of sewage loading. This nutrient may have originated from the oxidation of other nitrogen compounds and may be rapidly incorporated by microorganisms along the river (McClain et al., 1998). In addition, there are various sources of nitrate, such as the application of fertilizers, which makes it even more limited as a pollution indicator for sewage contamination (Seiler et al., 1999).

An increment of two times the concentrations of caffeine, nitrate, ammonia and phosphate was observed when comparing the concentrations measured in the dry season with those of the rainy season. This was expected since the water volume of Paquequer River is strongly reduced during the dry season but the discharge of wastewater remains constant. Similar results were found in the study by Viviano et al. (2014).

In Brazil, water pollution observed in urban areas involves mainly the inappropriate discharge of domestic wastewater and waste disposal and demographic growth. It is very common to observe the dumping of wastewater effluents direct into the rivers, lakes and seas. 
As a result, in many regions of Brazil, wastewater is considered to be the major source of water pollution. Furthermore, water resources are vulnerable to contamination by local land uses and especially by waste disposal. In addition to fast population growth, the reliance on septic systems and cesspools for domestic wastewater disposal as well as the practice of discharging treated wastewater to surface water provide significant contamination.

\subsubsection{Comparison between found and expected caffeine concentration in the Paquequer River}

The Paquequer River acts as a surface water collection system for inputs of wastewater without treatment along the city traverse. In order to evaluate the relationship between caffeine consumption by the population and the values found in the Paquequer River, estimates were calculated based on the following considerations: $i$ - in 2007, the population of Teresópolis was of 150.268 inhabitants and $83 \%$ of this population (124,722 inhabitants) lives in the urban area (IBGE, 2007); ii- the wastewater produced by $23.28 \%$ of this population is directly into the Paquequer River (CNM, 2000); iii- the consumption of caffeine per person per day is $171 \mathrm{mg}$ day $^{-1}$ (Camargo et al., 1999); iv- the waste produced by each person is $0.16 \mathrm{~m}^{3}$ day $^{-1}$; and $v$ - the amount of caffeine excreted by the human body non-metabolized is of 1 to $6 \%$ of the total ingested (Chen et al., 2002). The results are shown in Table 2.

Table 2. Comparison between found and expected caffeine concentrations in the Paquequer River based on the population variables.

\begin{tabular}{|c|c|}
\hline Population Variables & Information \\
\hline Estimated urban population ( $83 \%$ of the total) & 124,722 habitants \\
\hline $\begin{array}{l}\text { Population whose wastewater is discharged directly } \\
\text { into the water body or other sewer }\end{array}$ & $23.28 \%$ \\
\hline Daily caffeine consumption per person & $171 \mathrm{mg} \mathrm{day}^{-1}$ \\
\hline $\begin{array}{l}\text { Percentage of caffeine unmetabolized and excreted per } \\
\text { person }\end{array}$ & $1-6 \%$ \\
\hline $\begin{array}{l}\text { Total excreted caffeine (unmetabolized) daily per } \\
\text { person }\end{array}$ & $1.71-10.26 \mathrm{mg} \mathrm{day}^{-1}$ \\
\hline Sewage volume produced daily per person & $0.16 \mathrm{~m}^{3}$ day $^{-1}$ \\
\hline $\begin{array}{l}\text { Total caffeine dumped through the sewage daily, } \\
\text { considering the percentage of the population of } 23.8 \%\end{array}$ & 49,650 a $297,899 \mathrm{mg}$ \\
\hline $\begin{array}{l}\text { Estimated daily sewage volume entering the } \\
\text { Paquequer River }\end{array}$ & $19,956 \mathrm{~m}^{3} \mathrm{day}^{-1}$ \\
\hline $\begin{array}{l}\text { Estimated caffeine concentration into the Paquequer } \\
\text { River }\end{array}$ & $10.69-64.12 \mu \mathrm{g} \mathrm{L}^{-1}$ \\
\hline $\begin{array}{l}\text { Caffeine concentration measured in the Paquequer } \\
\text { River in the urban área }\end{array}$ & $3.85-47.45 \mu \mathrm{g} \mathrm{L}^{-1}$ \\
\hline
\end{tabular}

Taking these conditions into consideration, we can calculate: $i$ - the amount of non-metabolized caffeine excreted by the human organism ranges from 1.71 to 
$10.6 \mathrm{mg} \mathrm{person}^{-1}$ day $^{-1}$; ii- a total of 49,650 to $297,899 \mathrm{mg}$ of caffeine is introduced into the Paquequer River every day in Teresópolis; $i i i-$ the volume of untreated waste produced daily is $19,956 \mathrm{~m}^{3}$ day $^{-1}$; and $v i$ - the concentration in the waters should range from 10.69 to $64.12 \mu \mathrm{g} \mathrm{L}^{-1}$. The calculated value is very close to that found in this work, which was from 3.85 a $47.45 \mu \mathrm{g} \mathrm{L}^{-1}$.

\section{CONCLUSIONS}

The results found in this work support the use of caffeine as marker of wastewater contamination of surface waters independent of weather conditions. Increasing of caffeine concentrations in the Paquequer River was observed in both the dry and rainy seasons. Additionally, the results are in concordance with those theoretically calculated for Teresópolis. This means the analytical methodology used in the study produced creditable results.

The concentrations of caffeine measured in the Paquequer waters correlated with those of ammonia and phosphate, but not with those of nitrate, indicated its superiority as indicator of water contamination due to domestic wastes other than nitrate.

The presence of caffeine in water samples collected inside the National Park, a pristine environment, and the absence of any correlation with caffeine concentrations and those of ammonia and /or phosphate, indicates the need for additional work to identify the origin of such contamination. Also, the sources of caffeine, its stability, chemical behavior and availability in tropical environments are matter of concern.

\section{ACKNOWLEDGMENTS}

The authors would like to thank the Brazilian National Research Council for financial support and Dr. Josino Costa Moreira for his suggestions.

\section{REFERENCES}

AMERICAN PUBLIC HEALTH ASSOCIATION - APHA; AMERICAN WATER WORKS ASSOCIATION - AWWA; WATER ENVIRONMENT FEDERATION - WEF. Standard methods for the examination of water and wastewater. $22^{\text {nd }}$ ed. Washington, 2013.

BURGE, I. J.; POIGER, T.; MULlER, M. D.; BUSER, H.-R. Caffeine, an anthropogenic marker for wastewater contamination of surface waters. Environmental Science \& Technology, v. 37, n. 10, p. 691-700, 2003.

BRADLEY, P. M.; BARBER, L. B.; KOLPIN, D. W.; MCMAHON, P. B.; CHAPELLE, F. H. Biotransformation of caffeine, cotinine, and nicotine in stream sediments: Implications for use as wastewater indicators. Environmental Toxicology and Chemistry, v. 26, n. 6, p. 1116-1121, 2007.

CAHILL, J. D.; FURLONG, E. T.; BURKEHARDT, M. R.; KOLPIN, D.; ANDERSON. L. G. Determination of pharmaceutical compounds in surface- and ground-water samples by solid phase extraction and high-performance liquid chromatography - electrospray ionization mass spectrometry. Journal of Chromatography A, v. 1041, p. 171-180, 2004.

CAMARGO, M. C. R.; TOLEDO, M. C. F.; FARAH, H. G. Caffeine daily intake from dietary sources in Brazil. Food Additives and Contaminants, v. 16, n. 2, p. 19-87, 1999. 
CANTWELL, M. G.; KATZ, D. R.; SULLIVAN, J. C.; BORCI, T.; CHEN, R. F. Caffeine in Boston Harbor past and present, assessing its utility as a tracer of wastewatercontamination in an urban estuary. Marine Pollution Bulletin, v. 108, n. 1-2, p. 321-324, 2016.

CHEN, Z.; PAVELIC, P.; DILLON, P.; NAIDU, R. Determination of caffeine as a tracer of sewage effluent in natural waters by on-line solid-phase extraction and liquid chromatography with diode-array detection. Water Research, v. 36, n. 19, p. 4830-4838, 2002.

CONFEDERAÇÃO NACIONAL DE MUNICÍPIOS - CNM. Infra-estrutura: esgotamento sanitário de Teresópolis/RJ. 2000. Available in:

http://portal.cnm.org.br/v4/v11/infra/mu_infra_esgotamento.asp?iIdMun=100133086. Access in: 27 jan. 2017.

DANESHVAR, A.; ABOULFADL, K.; VIGLINO, L; BROSÉUS, R.; SAUVÉ, S.; MADOUX-HUMERY, A.-S. et al.Evaluating pharmaceuticals and caffeine as indicator of fecal contamination in drinking water sources of the Greater Montreal region. Chemosphere, v. 88, n. 1, p. 131-139, 2012.

FERREIRA, A. P. Caffeine as an environmental indicator for assessing urban aquatic ecosystems, Cadernos Saúde Pública, v. 21, n. 6, p. 1884-92, 2005.

GARDINALI, P. R.; ZHAO, X. Trace determination of caffeine in surface water samples by liquid chromatography-atmospheric pressure chemical ionization-mass spectrometry (LC-APCI-MS). Environment International, v. 28, n. 6, p. 521-528, 2002.

GLASSMEYER, S. T.; FURLONG, E. T.; KOLPIN, D.W.; CAHILL, J. D.; ZAUGG, S. D.; WERNER, S. L. et al. Transport of chemical and microbial compounds from known wastewater discharges: Potential for use as indicators of human fecal contamination. Environmental Science \& Technology, v. 39, n. 13, p. 5157-5169, 2005.

GOMEZ, M. J.; MARTINEZ BUENO, M. J.; LACORTE, S.; FERNANDEZ-ALBA, A. R.; AGUERA, A. Pilot survey monitoring pharmaceuticals and related compounds in a sewage treatment plant located on the Mediterranean coast. Chemosphere, v. 66, n. 6, 993-1002, 2007.

INSTITUTO BRASILEIRO DE GEOGRAFIA E ESTATÍSTICA - IBGE. Censo Demográfico. 2007. Available in: http://www.ibge.gov.br. Access in: 10 jun. 2016.

MACCLAIN, M. E.; BILBY, R. E.; FRANK, J. T. Nutrient cycles and responses to disturbance. In: NAIMAN, R. J.; BILBY, R. E. (Ed.). River Ecology and Management. New York: Springer, 1998. 705p.

MEYBECK, M. Global analysis of river systems: from Earth system controls to Anthropocene syndromes. Philosophical Transactions of the Royal Society of London B, v. 358, n. 21, p. 1935-1955, 2003.

MYERS, S. S.; GAFFIKIN, L.; GOLDEN, C. D.; OSTFELD, R. S.; REDFORD, K. H.; RICKETTS, T. H. et al. Human health impacts of ecosystem alteration. Proceedings of the National Academy of Sciences of the United States of America, v. 110, n. 47, p. 18753-18760, 2013. 
MONTAGNER, C. C.; JARDIM, W. F. Spatial and seasonal variations of pharmaceuticals and endocrine disruptors in the Atibaia River, São Paulo State (Brazil). Journal of the Brazilian Chemical Society, v. 22, n. 8, 2011.

PEELER, K.; OPSAHL, S.; CHANTON, J. Tracking anthropogenic inputs using caffeine, indicator bacteria, and nutrients in rural freshwater and urban marine systems. Environmental Science \& Technology, v. 40, n. 24, p. 7616-7622, 2006.

PESTA, D. H.; ANGADI, S. S.; BURTSCHER, M.; ROBERTS, C. K. The effects of caffeine, nicotine, ethanol, and tetrahydrocannabinol on exercise performance. Nutrition \& Metabolism, v. 10, n. 71, p. 15, 2013.

SANKARARAMAKRISHNAN, N.; GUO, Q. Chemical tracers as indicator of human fecal coliforms at storm water outfalls. Environmental International, v. 31, n. 8, p. 1133$1140,2005$.

SEILER, R. L.; ZAUGG, S. D.; THOMAS, J. M.; HOWCROFT, D. L. Caffeine and pharmaceuticals as indicators of waste water contamination in wells. Groundwater, v. 37, n. 3, 405-410, 1999.

SPENCE, P. L. Using caffeine as a water quality indicator in the ambient monitoring program for Third Fork Creek Watershed, Durham, North Carolina. Environmental Health Insights, v. 9, suppl. 2, p. 29-34, 2015.

SWARTZ, C. H.; REDDY, S.; BENOTTI, M. J.; YIN, H. F.; BARBER, L. B.; BROWNAWELL, B. J. et al. Steroid estrogens, nonylphenol ethoxylate metabolites, and other wastewater contaminants in groundwater affected by a residential septic system on Cape Cod, MA. Environmental Science \& Technology, v. 40, n. 16, p. 4894-4902, 2006.

TANG-LIU, D.; WILLIAMS, R.; RIEGELMAN, S. Disposition of caffeine and its metabolites in man. Journal of Pharmacology and Experimental Therapeutics, v. 224, n. 1, 180$185,1983$.

VERENITCH S. S.; LOWE, C. J.; MAZUMDER, A. Determination of acidic drugs and caffeine in municipal wastewaters and receiving waters by gas chromatography - ion trap tandem mass spectrometry. Journal of Chromatography A, v. 1116, n. 1-2, p. 193-203, 2006.

VIVIANO, G.; SALERNO, F.; MANFREDI, E. C.; POLESELlO, S.; VALSECCHI, S.; TARTARI, G. Surrogate measures for providing high frequency estimates of total phosphorus concentrations in urban watersheds. Water Research, v. 64, p. 265-277, 2014.

WEIGEL, S.; BERGER, U.; JENSEN, E.; KALLENBORN, R.; THORESEN, H.; HÜHNERFUSS, H. Determination of selected pharmaceuticals and caffeine in sewage and seawater from Tromsø/Norway with emphasis on ibuprofen and its metabolites. Chemosphere, v. 56, n. 6, p. 583-592, 2004. http://dx.doi.org/10.1016/j.chemosphere.2004.04.015 


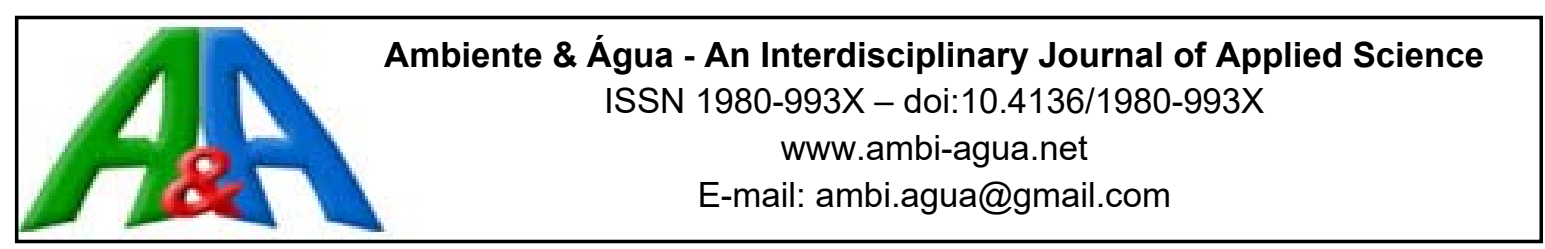

\title{
Emprego dos métodos expeditos de Chow Gumbel e Bell para formulação de equações de chuvas intensas - uma avaliação de desempenho
}

\author{
doi:10.4136/ambi-agua.2001 \\ Received: 09 Sep. 2016; Accepted: 20 Dec. 2016

\begin{abstract}
Karinnie Nascimento de Almeida; José Antonio Tosta dos Reis*; Antônio Sergio Ferreira Mendonça
\end{abstract} \\ Universidade Federal do Espírito Santo (UFES), Vitória, ES, Brasil \\ Departamento de Engenharia Ambiental \\ *Autor correspondente: e-mail: jatreis@gmail.com, \\ kaka.nascimento05@gmail.com, anserfm@terra.com.br
}

\section{RESUMO}

$\mathrm{O}$ adequado dimensionamento de estruturas de micro e macrodrenagem usualmente depende de vazões estimadas com auxílio de equações de chuvas intensas. Séries históricas de precipitações longas e representativas, obtidas através de pluviógrafos, permitem a conformação de equações que relacionem intensidade, duração e frequência de precipitações. Entretanto, em muitas regiões brasileiras não existem pluviógrafos em operação, ou as séries históricas de registros pluviográficos disponíveis são substancialmente mais curtas e menos confiáveis do que aquelas estabelecidas por pluviômetros. Neste contexto, recorrentemente são empregados métodos expeditos para obtenção de equações de chuvas intensas a partir de registros pluviométricos. O presente trabalho tem como objetivo avaliar o desempenho dos métodos expeditos de Chow-Gumbel e de Bell, quando da conformação de equações de chuvas intensas a partir de coeficientes de desagregação disponíveis na literatura técnica corrente. $\mathrm{O}$ teste F de variâncias, assumindo-se nível de significância de 95\%, foi empregado para comparar as respostas das equações de chuvas intensas produzidas pelos métodos expeditos com as respostas de equações de chuvas intensas estabelecidas a partir de registros pluviográficos. Como referência para a análise, foram consideradas equações de chuvas intensas disponíveis para o estado do Paraná. Os resultados indicaram que, para períodos de retorno superiores a 10 anos e durações superiores a 30 minutos, as equações de chuvas intensas determinadas pelos métodos expeditos produzem intensidades equivalentes àquelas determinadas por registros pluviográficos.

Palavras-chave: chuvas intensas, drenagem, equações intensidade-duração-frequência.

\section{Use of the expedited methods of Chow-Gumbel and Bell for formulation of intense rain equations - a performance evaluation}

\begin{abstract}
Adequate design of stormwater structures usually depends on peak runoff rates estimated with the aid of rainfall intensity-duration-frequency (i.d.f.) equations. Long and representative
\end{abstract}


historical precipitation series, obtained by pluviographs, are needed for the estimation of i.d.f. equation parameters. However, in many regions there are no pluviographs in operation or continuous rain gauge records obtained by using this kind of equipment are much shorter and less reliable than those obtained by using manual rain gauge equipment. In this context, expeditious methods are needed for obtaining intensity-duration-frequency equations from daily precipitation records. This study evaluates the performance of the Chow-Gumbel and Bell methods for the establishment of i.d.f. rainfall equations with usual conversion coefficients. The $\mathrm{F}$ test of variance, assuming a 95\% significance level, was used to compare the empirical methods' results with those obtained from pluviographic records. Available pluviographic and regular pluviometers data for stations located in Paraná, state in Brazil were considered. The results are equivalent for return periods greater than 10 years and for durations greater than 30 minutes.

Keywords: extreme rainfall, intensity-duration-frequency equations, urban drainage.

\section{INTRODUÇÃO}

O dimensionamento das diferentes estruturas que conformam os sistemas de micro e macrodrenagem (galerias de águas pluviais, bueiros, sarjetas e reservatórios de detenção em áreas urbanas, por exemplo) é usualmente conduzido a partir de vazões de projeto estimadas por meio de modelos chuva-vazão. O emprego de modelos chuva-vazão, por sua vez, depende de uma equação que relacione intensidade, duração e frequência de precipitações. Neste contexto, a determinação das equações de chuvas intensas constitui, portanto, aspecto central para os projetos de sistemas urbanos de drenagem pluvial (Fendrich, 1999).

Existindo uma rede confiável de pluviógrafos, operada durante período de tempo suficientemente longo e representativo, é possível a definição de relações entre intensidades, durações e frequências de precipitações. Constituem exemplos de equações de chuvas intensas desenvolvidas a partir de registros pluviográficos aquelas apresentadas por Reich (1963), Chen (1983), Kothyari e Garde (1992), Alila (2000), Silva et al. (2000), Falaguasta e Genovez (2003), Oliveira et al. (2005), Hadadin (2005), Back (2006), Singh e Zhang (2007), Garcia et al. (2011) e Fendrich (2011).

Para as situações nas quais não se dispõe de registros pluviográficos, a literatura técnica corrente apresenta diferentes métodos expeditos para a determinação de equações de chuvas intensas, a partir de séries históricas obtidas da operação de pluviômetros. Dentre os métodos expeditos, os métodos de Chow-Gumbel e Bell constituem alternativas que permitem a apropriação de chuvas de diferentes durações e frequências a partir das chuvas máximas anuais de um dia de duração.

No Brasil, em função da limitada disponibilidade de registros pluviográficos, os métodos de Chow Gumbel e Bell são recorrentemente empregados, como nos trabalhos de Almeida et al. (2015), Oliveira et al. (2000), Nali et al. (2007), Rodrigues et al. (2008), Oliveira et al. (2008), Coutinho et al. (2010), Back (2012) e Campos et al. (2014). No entanto, as equações estabelecidas a partir dos referidos métodos usualmente utilizam coeficientes de desagregação de chuvas que não foram localmente determinados. Ainda que diferentes autores (Reich, 1963; Bell, 1969; Genovez e Zuffo, 2000) sugiram que os coeficientes de desagregação se apresentem similares em diferentes partes de mundo, o emprego de coeficientes disponíveis na literatura técnica corrente conduz a intensidades pluviométricas estimadas a partir de equações produzidas sem adequadas calibração e validação.

Neste contexto, o presente trabalho teve como objetivo avaliar o desempenho de equações de chuvas intensas originalmente definidas por Almeida (2015) a partir dos métodos de 
Chow-Gumbel e de Bell, considerados coeficientes de desagregação usualmente empregados no Brasil. Para aplicação do método de Chow-Gumbel foram empregados os coeficientes de desagregação estabelecidos por Pfafstetter (1957) e Occhipinti e Santos (1966). O método de Bell, por sua vez, demandou coeficiente de desagregação sugerido por Righetto (1998). Para avaliação do desempenho das equações conformadas com auxílio dos referidos métodos expeditos foram consideradas, como referência, equações de chuvas intensas desenvolvidas a partir de registros de estações pluviográficas localizados no estado do Paraná.

\section{2. ÁREA DE ESTUDO}

O estado do Paraná está localizado na região sul do Brasil, entre $22^{\circ} 29^{\prime}$ e $26^{\circ} 43^{\prime}$ de latitudes sul e $48^{\circ} 05^{\prime}$ e $54^{\circ} 37^{\prime}$ de longitudes oeste. Segundo a classificação de Köppen, o clima do Paraná é dividido em Af (tropical) sempre úmido, sem estação seca e isento de geadas, na planície litorânea; $\mathrm{Cfa}$ (subtropical) com verões quentes, geadas pouco frequentes e chuvas no verão nas regiões norte, oeste e parte do sudoeste; $\mathrm{Cfb}$ (temperado) com verões frescos, sem estação seca definida, entre o primeiro, segundo e parte do terceiro planaltos (ITCF, 1987).

\section{MATERIAIS E MÉTODOS}

\subsection{Equações de chuvas intensas estabelecidas com auxílio de pluviógrafos.}

As equações de chuvas intensas estabelecidas a partir de registros pluviográficos e disponíveis para o estado que compõe a área de estudo foram estabelecidas por Fendrich (2011). As referidas equações, que constituíram as referências para a análise das respostas oferecidas pelas equações de chuvas intensas conformadas a partir dos métodos expeditos, juntamente com nome e localização das estações pluviográficas e períodos de monitoramento, estão reunidas no Quadro 1. A distribuição espacial das estações pluviográficas no estado do Paraná é apresentada por meio da Figura 1.

Quadro 1. Estações pluviográficas e equações de chuvas intensas disponíveis para o estado do Paraná.

\begin{tabular}{|c|c|c|c|c|c|c|}
\hline Estações & Município & $\begin{array}{l}\text { Altitude } \\
(\mathrm{m})\end{array}$ & $\begin{array}{l}\text { Latitude S } \\
\text { (Grau Minuto) }\end{array}$ & $\begin{array}{l}\text { Longitude W } \\
\text { (Grau Minuto) }\end{array}$ & Período & Equação \\
\hline Araucária & Araucária & 903 & $25^{\circ} 32^{\prime}$ & $49^{\circ} 23^{\prime}$ & 1989-1999 & $\mathrm{i}=\frac{2505,53 \cdot \mathrm{T}^{0,117}}{(\mathrm{t}+13)^{0,988}}$ \\
\hline Curitiba & Curitiba & 929 & $25^{\circ} 26^{\prime}$ & $49^{\circ} 58^{\prime}$ & $1921-1951$ & $\mathrm{i}=\frac{5950 \cdot \mathrm{T}^{0,217}}{(\mathrm{t}+26)^{1,15}}$ \\
\hline Ivaiporã & Ivaiporã & 650 & $24^{\circ} 15^{\prime}$ & $51^{\circ} 39^{\prime}$ & 1979-1994 & $i=\frac{676,71 \cdot T^{0,158}}{(t+1)^{0,726}}$ \\
\hline Londrina & Londrina & 585 & $23^{\circ} 18^{\prime}$ & $51^{\circ} 09^{\prime}$ & 1975-1985 & $i=\frac{3132,56 \cdot T^{0,093}}{(t+30)^{0,939}}$ \\
\hline Palmital & $\begin{array}{l}\text { Águas } \\
\text { Paraná }\end{array}$ & 840 & $24^{\circ} 53^{\prime}$ & $52^{\circ} 13^{\prime}$ & 1979-1990 & $i=\frac{1548,46 \cdot T^{0,130}}{(t+16)^{0,834}}$ \\
\hline $\begin{array}{c}\text { Porto } \\
\text { Amazonas }\end{array}$ & $\begin{array}{c}\text { Porto } \\
\text { Amazonas }\end{array}$ & 973 & $25^{\circ} 33^{\prime}$ & $49^{\circ} 53^{\prime}$ & 1978-1992 & $\mathrm{i}=\frac{2543,31 \cdot \mathrm{T}^{0,196}}{(\mathrm{t}+27)^{0,952}}$ \\
\hline $\begin{array}{l}\text { Prado } \\
\text { Velho }\end{array}$ & Curitiba & 884 & $25^{\circ} 27^{\prime}$ & $49^{\circ} 15^{\prime}$ & 1981-1999 & $\mathrm{i}=\frac{3221,07 \cdot \mathrm{T}^{0,258}}{(\mathrm{t}+26)^{1,010}}$ \\
\hline Tibagi & Tibagi & 720 & $24^{\circ} 30^{\prime}$ & $50^{\circ} 24^{\prime}$ & 1984-1993 & $\mathrm{i}=\frac{1592,58 \cdot \mathrm{T}^{0,136}}{(\mathrm{t}+11)^{0,882}}$ \\
\hline Tomazina & Tomazina & 483 & $23^{\circ} 46^{\prime}$ & $49^{\circ} 57^{\prime}$ & 1979-1990 & $\mathrm{i}=\frac{2676,7 \cdot \mathrm{T}^{0,149}}{(\mathrm{t}+29)^{0,931}}$ \\
\hline
\end{tabular}

Fonte: Fendrich. (2011). 


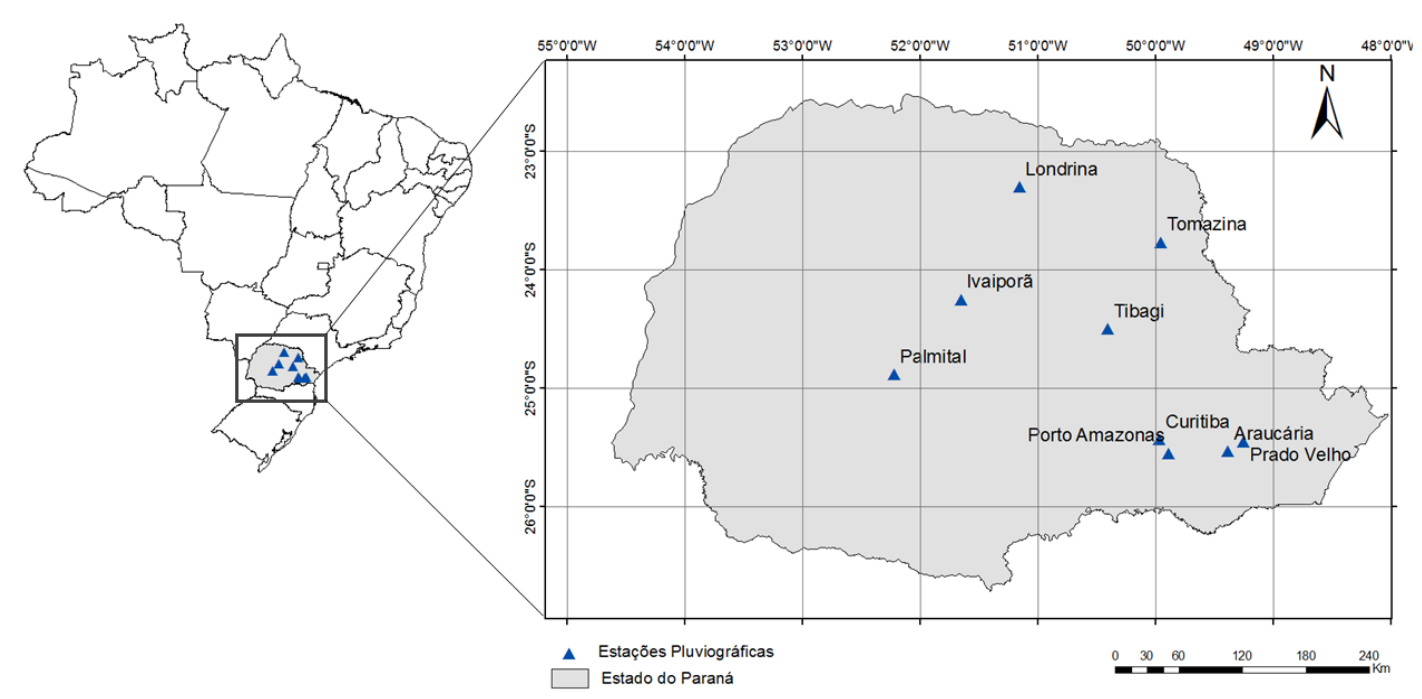

Figura 1. Localização das estações pluviográficas no estado do Paraná.

\subsection{Registros pluviométricos}

Para o estabelecimento de equações de chuvas intensas desenvolvidas a partir dos métodos expeditos de Chow-Gumbel e de Bell foram utilizados registros pluviométricos disponíveis na base de dados gerenciada pela Agência Nacional das Águas (ANA). Foram manipuladas séries históricas consistidas de totais diários de precipitação, registrados em estações pluviométricas instaladas nas mesmas coordenadas geográficas das estações pluviográficas e empregados por Fendrich (2011).

Para a manipulação das séries históricas selecionadas correspondentes aos mesmos períodos analisados no Quadro 1, foi empregado o programa computacional HIDRO, programa de domínio público produzido e disponibilizado pela ANA.

\subsection{Método expedito de Chow-Gumbel}

A aplicação do Método de Chow-Gumbel, apresentado e discutido por CETESB (1986), envolveu as seguintes etapas:

- Seleção das máximas precipitações anuais de 1 dia duração;

- Análise de frequências dos totais precipitados com ajuste da distribuição probabilística de Gumbel à série de máximas precipitações anuais de 1 dia. Dessa forma, foram estimadas as precipitações máximas anuais de 1 dia associadas aos períodos de retorno de 2, 10, 20 e 50 anos;

- Obtenção, a partir dos totais precipitados de 1 dia, das precipitações para durações de 24 horas. Conforme sugerem Occhipinti e Santos (1966), a relação entre as chuvas máximas de 24 horas e 1 dia oscila em torno do valor de 1,14, fator de conversão utilizado no presente trabalho;

É relevante observar que o fator de conversão proposto por Occhipinti e Santos (1966) foi estabelecido a partir da avaliação de precipitações registradas na cidade de São Paulo, não apresentando, portanto, qualquer relação com os registros das estações pluviográficas analisadas por Fendrich (2011).

- Determinação, a partir da avaliação da chuva com duração de 24 horas, das chuvas com mesma frequência de ocorrência, mas com menor duração. Foram empregados, nesta etapa do trabalho, os fatores de conversão apresentados na Tabela 1;

Os fatores de conversão reunidos na Tabela 1 foram estabelecidos por Pfafstetter (1957) a partir da análise de 98 estações pluviográficas instaladas no território brasileiro, incluindo-se 
neste conjunto quatro estações localizadas no estado do Paraná (estações de Curitiba, Jacarezinho, Paranaguá e Ponta Grossa). É relevante observar que apenas uma estação (Curitiba), dentre aquelas analisadas por Pfafstetter (1957), foi também considerada no estudo conduzido por Fendrich (2011). Desta forma, em função dos diferentes conjuntos de estações pluviográficas analisadas, entende-se que os coeficientes propostos por Pfafstetter (1957) não podem ser considerados particulares para o estado do Paraná.

- Estabelecidas as alturas pluviométricas associadas a diferentes períodos de retorno e durações, as intensidades foram produzidas como simples relações entre alturas e durações;

- Definição de equações de chuvas intensas no formato estabelecido pela Equação (1).

$$
\mathrm{i}=\frac{\left(\mathrm{K} \cdot \mathrm{T}^{\mathrm{a}}\right)}{(\mathrm{t}+\mathrm{b})^{\mathrm{c}}}
$$

em que:

i, representa a intensidade máxima ( $\mathrm{mm} /$ minuto),

$\mathrm{T}$, o período de retorno (anos),

$\mathrm{T}$, a duração (minutos) e

$\mathrm{K}$, a, b e c são os parâmetros que localmente se deseja determinar.

O ajuste do modelo estabelecido por meio da Equação 1 foi realizado com auxílio da Programação Não Linear (PNL), aplicada com auxílio do programa Solver, disponível na Planilha Microsoft Excel. Para aplicação da PNL estabeleceu-se uma função objetivo (Equação 2).

$$
\min f_{o}=\sum_{\mathrm{T}} \sum_{\mathrm{t}}\left(\mathrm{i}_{\text {Método }}-\mathrm{i}_{\text {Equação }}\right)
$$

Buscou-se minimizar o erro total entre os valores de intensidade pluviométrica estimados a partir das etapas de 1 a 5 (imétodo) e as intensidades estimadas com auxílio da Equação 1 (iEquação). Os parâmetros $\mathrm{k}, \mathrm{a}, \mathrm{b}$ e c constituíram as variáveis de decisão do problema de otimização, com valores pertencentes ao conjunto $\mathcal{R}+$.

Para a garantia de determinação do ótimo global associado à expressão (02) foram testados diferentes valores iniciais para os parâmetros a, b, c e k. O processo de otimização conduzido com auxílio da PNL foi repetido, no mínimo, 10 (dez) vezes.

Tabela 1. Relação entre as alturas pluviométricas para precipitações de mesma frequência e diferentes durações.

\begin{tabular}{cc}
\hline $\begin{array}{c}\text { Relação entre as alturas } \\
\text { pluviométricas }\end{array}$ & $\begin{array}{c}\text { Coeficientes de } \\
\text { conversão }\end{array}$ \\
\hline $5 \mathrm{~min} / 30 \mathrm{~min}$ & 0,34 \\
$10 \mathrm{~min} / 30 \mathrm{~min}$ & 0,54 \\
$15 \mathrm{~min} / 30 \mathrm{~min}$ & 0,70 \\
$20 \mathrm{~min} / 30 \mathrm{~min}$ & 0,81 \\
$25 \mathrm{~min} / 30 \mathrm{~min}$ & 0,91 \\
$30 \mathrm{~min} / 1 \mathrm{~h}$ & 0,74 \\
$1 \mathrm{~h} / 24 \mathrm{~h}$ & 0,42 \\
$6 \mathrm{~h} / 24 \mathrm{~h}$ & 0,72 \\
\hline
\end{tabular}

Fonte: Pfafstetter (1967). 


\subsection{Método expedito de Bell}

O Método de Bell estima a altura pluviométrica produzida por uma precipitação com duração t e período de retorno $\mathrm{T}$, a partir de uma chuva intensa padrão de 60 minutos de duração e 2 anos de período de retorno (h60,2), conforme Equação 3.

$$
\mathrm{h}_{\mathrm{t}, \mathrm{T}}=\left(a_{0} \cdot \ln \mathrm{T}+a_{1}\right) \cdot\left(a_{2} \cdot \mathrm{t}^{\mathrm{b}}-a_{3}\right) \cdot \mathrm{h}_{60,2}
$$

em que:

a0, $a_{1}, a_{2}, a_{3}$ e b constituem parâmetros regionais.

Conforme Righetto (1998), os referidos parâmetros foram estimados a partir da manipulação de postos pluviográficos instalados em diferentes regiões do País, permitindo a conformação da Equação 4.

$$
h_{t, T}=(0,31 \cdot \ln T+0,70) \cdot\left(0,38 \cdot t^{0,31}-0,39\right) \cdot h_{60,2}
$$

Righetto (1998) sugere, adicionalmente, que o valor de $\mathrm{h}_{60,2}$ pode ser obtido a partir da precipitação máxima diária associada ao período de retorno de 2 anos $\left(\mathrm{h}_{\mathrm{dia}, 2}\right)$, conforme Equação 5.

$$
\mathrm{h}_{60,2} \cong 0,51 . \mathrm{h}_{\mathrm{dia}, 2}
$$

É relevante observar que o fator de conversão $(0,51)$ que conforma a última Equação 5 foi estabelecido a partir da avaliação de precipitações registradas no estado de São Paulo, não apresentando, portanto, qualquer relação com os registros das estações pluviográficas analisadas por Fendrich (2011).

No presente trabalho, a precipitação máxima diária associada ao período de retorno de 2 anos foi apropriada a partir do emprego da distribuição probabilística de Gumbel.

\subsection{Análise estatística}

Para a comparação das respostas oferecidas pelas equações de chuvas intensas determinadas pelos métodos de Chow-Gumbel e de Bell com as equações de chuvas intensas determinadas por Fendrich (2011) foi empregado o teste $\mathrm{F}$ de variância, detalhadamente discutido por Levine et al. (2005). Este procedimento reproduz a abordagem empregada por Almeida et al. (2015), quando da avaliação de respostas de equações de chuvas intensas estabelecidas pelos métodos de Chow-Gumbel e de Bell para o estado da Bahia. A Equação 6 define a estatística do teste $\mathrm{F}$ para a equivalência entre duas variâncias $\left(\mathrm{F}_{\text {est }}\right)$, assumindo-se a independência entre as séries de dados a partir das quais as variâncias foram apropriadas.

$$
\mathrm{F}_{\mathrm{est}}=\frac{\mathrm{S}_{1}^{2}}{\mathrm{~S}_{2}^{2}}
$$

em que:

$S_{1}^{2}$ e $S_{2}^{2}$ representam as variâncias da primeira e segunda amostras, respectivamente.

A primeira amostra é definida como a que possui a maior variância (Levine et al., 2005). Neste estudo, as amostras foram formadas por intensidades de precipitações estimadas pelas diferentes equações de chuvas intensas, para um mesmo valor de duração (t) e período de retorno $(\mathrm{T})$.

Para um determinado nível de significância, a hipótese nula de igualdade entre variâncias de duas amostras é conformada pela Equação 7. A hipótese alternativa de que as variâncias para 
as duas populações não são iguais, por sua vez, é definida pela Equação 8. A hipótese nula será rejeitada se a estatística do teste $\left(\mathrm{F}_{\text {est }}\right)$ for maior do que o valor crítico da distribuição $\mathrm{F}\left(\mathrm{F}_{\text {crítico }}\right)$. Portanto, a regra de decisão é estabelecida pela Equação 9.

$$
\begin{aligned}
& \mathrm{H}_{0}: \mathrm{S}_{1}^{2}=\mathrm{S}_{2}^{2} \\
& \mathrm{H}_{1}: \mathrm{S}_{1}^{2} \neq \mathrm{S}_{2}^{2}
\end{aligned}
$$

$$
\text { Rejeitar } \mathrm{H}_{0} \text { se } \mathrm{F}_{\text {est }}>\mathrm{F}_{\text {crítico }}
$$

A aplicação do teste $\mathrm{F}$ exigiu, adicionalmente, que a probabilidade de $\mathrm{Fest}$ ser menor que o

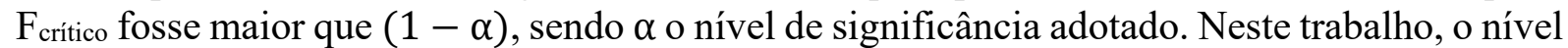
de significância foi de $95 \%$.

A comparação das respostas das equações de chuvas intensas foi realizada para grupos de intensidades pluviométricas associadas aos períodos de retorno de 2, 10, 20 e 50 anos e durações de 5, 30, 60, 240, 720 e 1440 minutos.

\section{RESULTADOS E DISCUSSÃO}

O Quadro 2 reúne as equações de chuvas intensas estabelecidas para o estado do Paraná a partir dos métodos expeditos de Chow-Gumbel e de Bell.

Quadro 2. Equações de chuvas intensas estabelecidas para o estado do Paraná pelos métodos expeditos de Chow-Gumbel e de Bell.

\begin{tabular}{|lll|}
\hline \multirow{2}{*}{ Estação } & \multicolumn{1}{c|}{ Método expedito de determinação das equações IDF } \\
\cline { 2 - 3 } Chow-Gumbel & \multicolumn{1}{c|}{ Bell } \\
\hline Curitiba & $\mathrm{i}=\frac{622,829 \cdot \mathrm{T}^{0,168}}{(\mathrm{t}+2,461)^{1,704}}$ & $\mathrm{~h}_{\mathrm{t}, \mathrm{T}}=(0,31 \cdot \ln \mathrm{T}+0,7) \cdot\left(0,38 \cdot \mathrm{t}^{0,31}-0,39\right) \cdot 37,46$ \\
Ivaiporã & $\mathrm{i}=\frac{569,265 \cdot \mathrm{T}^{0,179}}{(\mathrm{t}+2,444)^{1,704}}$ & $\mathrm{~h}_{\mathrm{t}, \mathrm{T}}=(0,31 \cdot \ln \mathrm{T}+0,7) \cdot\left(0,38 \cdot \mathrm{t}^{0,31}-0,39\right) \cdot 33,88$ \\
Londrina & $\mathrm{i}=\frac{731,645 \cdot \mathrm{T}^{0,188}}{(\mathrm{t}+2,464)^{1,705}}$ & $\mathrm{~h}_{\mathrm{t}, \mathrm{T}}=(0,31 \cdot \ln \mathrm{T}+0,7) \cdot\left(0,38 \cdot \mathrm{t}^{0,31}-0,39\right) \cdot 42,10$ \\
Palmital & $\mathrm{i}=\frac{760,765 \cdot \mathrm{T}^{0,185}}{(\mathrm{t}+2,485)^{1,706}}$ & $\mathrm{~h}_{\mathrm{t}, \mathrm{T}}=(0,31 \cdot \ln \mathrm{T}+0,7) \cdot\left(0,38 \cdot \mathrm{t}^{0,31}-0,39\right) \cdot 43,88$ \\
Porto Amazonas & $\mathrm{i}=\frac{887,594 \cdot \mathrm{T}^{0,135}}{(\mathrm{t}+2,418)^{1,704}}$ & $\mathrm{~h}_{\mathrm{t}, \mathrm{T}}=(0,31 \cdot \ln \mathrm{T}+0,7) \cdot\left(0,38 \cdot \mathrm{t}^{0,31}-0,39\right) \cdot 54,20$ \\
Prado Velho & $\mathrm{i}=\frac{816,563 \cdot \mathrm{T}^{0,182}}{(\mathrm{t}+2,468)^{1,705}}$ & $\mathrm{~h}_{\mathrm{t}, \mathrm{T}}=(0,31 \cdot \ln \mathrm{T}+0,7) \cdot\left(0,38 \cdot \mathrm{t}^{0,31}-0,39\right) \cdot 47,66$ \\
Tibagi & $\mathrm{i}=\frac{657,257 \cdot \mathrm{T}^{0,175}}{(\mathrm{t}+2,481)^{1,705}}$ & $\mathrm{~h}_{\mathrm{t}, \mathrm{T}}=(0,31 \cdot \ln \mathrm{T}+0,7) \cdot\left(0,38 \cdot \mathrm{t}^{0,31}-0,39\right) \cdot 39,00$ \\
Tomazina & $\mathrm{i}=\frac{639,072 \cdot \mathrm{T}^{0,135}}{(\mathrm{t}+2,446)^{1,704}}$ & $\mathrm{~h}_{\mathrm{t}, \mathrm{T}}=(0,31 \cdot \ln \mathrm{T}+0,7) \cdot\left(0,38 \cdot \mathrm{t}^{0,31}-0,39\right) \cdot 38,93$ \\
& $\mathrm{i}=\frac{667,466 \cdot \mathrm{T}^{0,131}}{(\mathrm{t}+2,456)^{1,708}}$ & $\mathrm{~h}_{\mathrm{t}, \mathrm{T}}=(0,31 \cdot \ln \mathrm{T}+0,7) \cdot\left(0,38 \cdot \mathrm{t}^{0,31}-0,39\right) \cdot 40,07$ \\
\hline
\end{tabular}

Para a apropriação das intensidades pluviométricas a partir das equações estabelecidas pelo método de Bell, os valores de altura pluviométrica $(\mathrm{h})$ foram divididos pelos valores de duração (t). Nas equações reunidas no Quadro 2, as intensidades pluviométricas (i) foram obtidas em 
mm.minuto ${ }^{-1}$ a partir de períodos de retorno $(\mathrm{T})$ indicados em anos, alturas pluviométricas em mm e durações em minutos.

Nas Tabelas 2 e 3 estão sumarizados os resultados da aplicação do Teste F de variância para comparação das respostas produzidas pelas equações de Fendrich (2011) e aquelas estabelecidas pelos métodos de Chow-Gumbel e Bell para o estado do Paraná. Nas referidas tabelas, $\mathrm{F}_{\text {est }}$ representa o parâmetro apropriado quando do emprego do teste $\mathrm{F}$ de variância, cujo

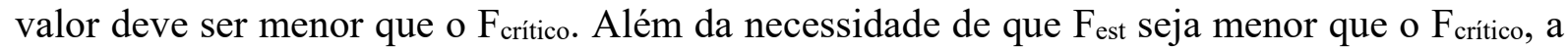
probabilidade de $F_{\text {est }}$ ser menor que o $F_{\text {crítico }}\left(P\left(F_{\text {est }} \leq F_{\text {crítico }}\right)\right)$ deve ser maior que $(1-\alpha)$. Nas tabelas, as células em destaque indicam as combinações de chuvas intensas que se apresentaram equivalentes.

Tabela 2. Resultado da aplicação do teste $\mathrm{F}$ de variância para intensidades pluviométricas associadas aos períodos de retorno de 2 anos e 10 anos.

\begin{tabular}{|c|c|c|c|c|c|c|c|}
\hline \multirow{3}{*}{ Duração (min) } & \multirow{3}{*}{ Métodos pareados } & \multicolumn{6}{|c|}{ Período de Retorno } \\
\hline & & \multicolumn{3}{|c|}{2 anos } & \multicolumn{3}{|c|}{10 anos } \\
\hline & & $\mathrm{F}_{\text {est }}$ & $\mathrm{F}_{\text {crítico }}$ & $\mathrm{P}\left(\mathrm{F}_{\text {est }} \leq \mathrm{F}_{\text {crítico }}\right)$ & $\mathrm{F}_{\text {est }}$ & $\mathrm{F}_{\text {crítico }}$ & $\mathrm{P}\left(\mathrm{F}_{\text {est }} \leq \mathrm{F}_{\text {critico }}\right)$ \\
\hline \multirow{3}{*}{5} & Gumbel - Bell & 1,19 & 3,44 & 0,41 & 1,18 & 3,44 & 0,41 \\
\hline & Bell - Fendrich (2011) & 3,80 & 3,44 & 0,04 & 2,67 & 3,44 & 0,09 \\
\hline & Gumbel - Fendrich (2011) & 3,19 & 3,44 & 0,06 & 2,26 & 3,44 & 1,14 \\
\hline \multirow{3}{*}{30} & Gumbel - Bell & 1,17 & 3,44 & 0,41 & 1,16 & 3,44 & 0,42 \\
\hline & Bell - Fendrich (2011) & 3,38 & 3,44 & 0,05 & 3,69 & 3,44 & 0,04 \\
\hline & Gumbel - Fendrich (2011) & 3,96 & 3,44 & 0,03 & 4,28 & 3,44 & 0,03 \\
\hline \multirow{3}{*}{60} & Gumbel - Bell & 1,14 & 3,44 & 0,43 & 1,12 & 3,44 & 0,44 \\
\hline & Bell - Fendrich (2011) & 1,26 & 3,44 & 0,37 & 1,84 & 3,44 & 0,20 \\
\hline & Gumbel - Fendrich (2011) & 1,44 & 3,44 & 0,31 & 2,06 & 3,44 & 0,16 \\
\hline \multirow{3}{*}{240} & Gumbel - Bell & 1,22 & 3,44 & 0,39 & 1,22 & 3,44 & 0,39 \\
\hline & Bell - Fendrich (2011) & 1,13 & 3,44 & 0,43 & 1,55 & 3,44 & 0,27 \\
\hline & Gumbel - Fendrich (2011) & 1,38 & 3,44 & 0,33 & 1,28 & 3,44 & 0,37 \\
\hline \multirow{3}{*}{720} & Gumbel - Bell & 1,18 & 3,44 & 0,41 & 1,51 & 3,44 & 0,29 \\
\hline & Bell - Fendrich (2011) & 1,27 & 3,44 & 0,37 & 1,64 & 3,44 & 0,25 \\
\hline & Gumbel - Fendrich (2011) & 1,50 & 3,44 & 0,29 & 1,09 & 3,44 & 0,45 \\
\hline \multirow{3}{*}{1440} & Gumbel - Bell & 2,38 & 3,44 & 0,12 & 1,83 & 3,44 & 0,21 \\
\hline & Bell - Fendrich (2011) & 1,45 & 3,44 & 0,31 & 1,37 & 3,44 & 0,33 \\
\hline & Gumbel - Fendrich (2011) & 3,46 & 3,44 & 0,05 & 1,33 & 3,44 & 0,35 \\
\hline
\end{tabular}

A simples inspeção das Tabelas 2 e 3 permite observar que a aplicação do teste $\mathrm{F}$ de variância indicou, para a área de estudo, a) equivalência entre as intensidades pluviométricas estimadas pelos métodos de Chow-Gumbel e Bell, para quaisquer durações ou períodos de retorno considerados e b) equivalência entre intensidades estimadas pelo método de ChowGumbel e aquelas apropriadas com auxílio das equações estabelecidas por Fendrich (2011) para durações superiores a 30 minutos, independentemente do períodos de retorno avaliado. 
Tabela 3. Resultado da aplicação do teste $\mathrm{F}$ de variância para intensidades pluviométricas associadas aos períodos de retorno de 20 anos e 50 anos.

\begin{tabular}{|c|c|c|c|c|c|c|c|}
\hline \multirow{3}{*}{ Duração (min) } & \multirow{3}{*}{ Métodos pareados } & \multicolumn{6}{|c|}{ Período de Retorno } \\
\hline & & \multicolumn{3}{|c|}{20 anos } & \multicolumn{3}{|c|}{50 anos } \\
\hline & & $\mathrm{F}_{\text {est }}$ & $\mathrm{F}_{\text {crítico }}$ & $\mathrm{P}\left(\mathrm{F}_{\text {est }} \leq \mathrm{F}_{\text {crítico }}\right)$ & $\mathrm{F}_{\text {est }}$ & $\mathrm{F}_{\text {crítico }}$ & $\mathrm{P}\left(\mathrm{F}_{\text {est }} \leq \mathrm{F}_{\text {crítico }}\right)$ \\
\hline \multirow{3}{*}{5} & Gumbel - Bell & 1,23 & 3,44 & 0,39 & 1,30 & 3,44 & 0,36 \\
\hline & Bell - Fendrich (2011) & 2,61 & 3,44 & 0,10 & 2,75 & 3,44 & 0,09 \\
\hline & Gumbel - Fendrich (2011) & 2,12 & 3,44 & 0,15 & 2,11 & 3,44 & 0,15 \\
\hline \multirow{3}{*}{30} & Gumbel - Bell & 1,21 & 3,44 & 0,40 & 1,28 & 3,44 & 0,37 \\
\hline & Bell - Fendrich (2011) & 2,40 & 3,44 & 0,12 & 1,44 & 3,44 & 0,31 \\
\hline & Gumbel - Fendrich (2011) & 2,90 & 3,44 & 0,08 & 1,84 & 3,44 & 0,20 \\
\hline \multirow{3}{*}{60} & Gumbel - Bell & 1,17 & 3,44 & 0,41 & 1,24 & 3,44 & 0,39 \\
\hline & Bell - Fendrich (2011) & 1,64 & 3,44 & 0,25 & 1,23 & 3,44 & 0,39 \\
\hline & Gumbel - Fendrich (2011) & 1,92 & 3,44 & 0,19 & 1,52 & 3,44 & 0,28 \\
\hline \multirow{3}{*}{240} & Gumbel - Bell & 1,17 & 3,44 & 0,42 & 1,11 & 3,44 & 0,45 \\
\hline & Bell - Fendrich (2011) & 1,67 & 3,44 & 0,24 & 1,63 & 3,44 & 0,25 \\
\hline & Gumbel - Fendrich (2011) & 1,44 & 3,44 & 0,31 & 1,48 & 3,44 & 0,30 \\
\hline \multirow{3}{*}{720} & Gumbel - Bell & 1,44 & 3,44 & 0,31 & 1,37 & 3,44 & 0,33 \\
\hline & Bell - Fendrich (2011) & 2,04 & 3,44 & 0,17 & 2,00 & 3,44 & 0,17 \\
\hline & Gumbel - Fendrich (2011) & 1,41 & 3,44 & 0,32 & 1,46 & 3,44 & 0,30 \\
\hline \multirow{3}{*}{1440} & Gumbel - Bell & 1,70 & 3,44 & 0,23 & 1,61 & 3,44 & 0,26 \\
\hline & Bell - Fendrich (2011) & 1,65 & 3,44 & 0,25 & 1,94 & 3,44 & 0,18 \\
\hline & Gumbel - Fendrich (2011) & 1,03 & 3,44 & 0,48 & 1,20 & 3,44 & 0,40 \\
\hline
\end{tabular}

Já a equivalência entre as intensidades pluviométricas estimadas a partir das equações definidas pelos métodos de Bell e por Fendrich (2011) foi observada para todas as durações, considerados os períodos de retorno de 20 e 50 anos. Para o período de retorno de 2 anos, não foi verificada equivalência para a duração de 5 minutos. Para o período de retorno de 10 anos, a equivalência entre intensidades não foi verificada para duração de 30 minutos. É relevante observar, no entanto, que para estes períodos de retorno ( 2 e 10 anos), o valor de $\mathrm{P}\left(\mathrm{F}_{\text {est }} \leq \mathrm{F}_{\text {crítico }}\right)$ assumiu valor substancialmente próximo $\left(\mathrm{P}\left(\mathrm{F}_{\text {est }} \leq \mathrm{F}_{\text {crítico }}\right)=0,04\right)$ do limite estabelecido para garantia de equivalência $(1-\alpha=0,05)$. Neste contexto, eventual flexibilização no nível de significância conduziria à equivalência entre as referidas intensidades.

Assim como observado por Almeida, Reis e Mendonça (2015), quando do estabelecimento de equações de chuvas intensas para o estado da Bahia, as intensidades pluviométricas estimadas pelos métodos de Chow-Gumbel e Bell apresentaram-se equivalentes, ao nível de significância de $95 \%$, para quaisquer durações ou períodos de retorno avaliados. Os referidos autores indicaram, no entanto, que a equivalência entre as equações conformadas com métodos de Chou-Gumbel e de Bell e as equações estabelecidas a partir de registros pluviográfícos foi verificada apenas para períodos de retorno superiores a 10 anos e durações maiores que 30 minutos. 
É relevante observar que, em projetos de drenagem pluvial, a duração da chuva é, usualmente, o tempo de concentração da bacia objeto da atuação do sistema de drenagem. Desta forma, excluindo-se bacias de pequenas dimensões (para as quais os tempos de concentração seriam inferiores a 30 minutos), os métodos expeditos podem constituir alternativa útil para apropriação das chuvas de projeto. Também constitui prática dos projetos de drenagem a adoção de intensidades pluviométricas associadas a períodos de retorno de 20 anos ou mais, frequência de ocorrência de precipitações para a qual os métodos expeditos também se apresentaram como alternativa consistente.

\section{CONCLUSÕES}

O presente estudo teve como principal objetivo avaliar o desempenho de equações de chuvas intensas definidas a partir dos métodos de Chow-Gumbel e Bell, métodos expeditos que envolvem o emprego de fatores de conversão entre chuvas de diferentes durações e que são recorrentemente empregados em avaliações de chuvas intensas em todo o território brasileiro. Como referência para avaliação do desempenho das referidas equações e, portanto, da consistência dos fatores de conversão que envolvem, foram consideradas equações de chuvas intensas desenvolvidas para o estado do Paraná a partir da análise de registros pluviográficos.

As principais conclusões do presente estudo podem ser assim sumarizadas:

- As intensidades pluviométricas estimadas para o estado do Paraná por equações desenvolvidas com o emprego dos métodos de Chow-Gumbel e de Bell apresentaram-se equivalentes ao nível de significância de $95 \%$, independentemente da duração ou período de retorno avaliados;

- As intensidades estimadas por equações definidas com o auxílio dos métodos de ChowGumbel e Bell apresentaram-se equivalentes àquelas estimadas a partir de equações conformadas com registros pluviográficos de chuvas com durações superiores a 30 minutos e períodos de retorno compreendidos entre 2 e 50 anos.

\section{REFERÊNCIAS}

ALILA, Y. Regional rainfall depth duration frequency equations for Canada. Water Resources Research, v. 36, n. 7, p. 1767-1778, 2000. 10.1029/2000WR900046

ALMEIDA, K. N. Avaliação do desempenho dos métodos expeditos de determinação de equações de intensidade-duração-frequência. 2015. 75f. Monografia (Graduação em Engenharia Ambiental) - Departamento de Engenharia Ambiental, Universidade Federal do Espírito Santo, Vitória, 2015.

ALMEIDA, K. N.; REIS, J. A. T.; MENDONÇA, A. S. F. Avaliação do desempenho dos métodos expeditos de determinação de equações de chuvas intensas. Revista Brasileira de Ciências Ambientais, v. 35, p. 63-77, 2015.

BACK, A. J. Relações de intensidade-duração-frequência de chuvas intensas de Chapecó, Estado de Santa Catarina. Revista Acta Scientiarum, v. 28, n. 4, p. 575-581, 2006.

BACK, A. J.; OLIVEIRA, J. L. R.; HENN, A. Relações entre precipitações intensas de diferentes durações para desagregação da chuva diária em Santa Catarina. Revista Brasileira de Engenharia Agrícola e Ambiental, v. 16, n. 4, p. 391-398, 2012. 
BELL, F. C. Generalized rainfall-duration-frequency relationships. Journal of the Hydraulics Division, v. 95, n. HY1, p. 311-327, 1969.

CAMPOS, A.R.; SANTOS, G. G.; SILVA, S.B.C.; FILHO, J. I.; LOURA. D. S. Equações de intensidade-duração-frequência de chuva para o estado do Piauí. Revista Ciência e Agronomia, v. 45, n. 3, p. 488-498, 2014.

CETESB - COMPANHIA DE TECNOLOGIA DE SANEAMENTO AMBIENTAL. Drenagem Urbana - Manual de Projeto. São Paulo: CETESB/ASCETESB, 1986.

CHEN, C. Rainfall intensity-duration-frequency formulas. Journal of Hydraulic Engineering, v. 109, n. 12, p. 1603-1621, 1983. http://dx.doi.org/10.1061/(ASCE)07339429(1983)109:12(1603)

COUTINHO, A. P.; SILVA, F. B.; SILVA, R. O.; ANTONINO, A. C. D.; MONTENEGRO, S. M. G. L. Determinação de equações de chuvas intensas para municípios das mesorregiões do estado de Pernambuco com dados pluviométricos In: SIMPÓSIO DE RECURSOS HÍDRICOS DO NORDESTE, 10., 2010, Fortaleza. Anais... Fortaleza: ABRH, 2010. 1 CD-ROM.

FALAGUASTA, L. N.; GENOVEZ, A. M. Equações de chuvas intensas para os estados de São Paulo e Paraná. Revista Brasileira de Recursos Hídricos, v. 8, n. 3, p. 169-176, 2003. http://unicamp.sibi.usp.br/handle/SBURI/13414

FENDRICH, R. Chuvas intensas para obras de drenagem no estado do Paraná. Curitiba: Gráfica Vicentina Editora, 2011.

FENDRICH, R. Importância das Equações de Chuvas Intensas na Drenagem Urbana. In: SIMPÓSIO BRASILEIRO DE RECURSOS HÍDRICOS, 13., 1999, Belo Horizonte. Anais... Belo Horizonte: ABRH, 1999. 1 CD-ROM.

GARCIA, S. S.; AMORIM, R. S. S.; COUTO, E. G.; STOPA, W. H. Determinação de equações intensidade-duração-frequência para três estações meteorológicas do estado do Mato Grosso. Revista Brasileira de Engenharia Agrícola e Ambiental, v. 15, n. 6, p. 575$581,2011$.

GENOVEZ, A. M.; ZUFFO, A. C. Chuvas intensas no estado de São Paulo. Revista Brasileira de Recursos Hídricos. v .5, n. 3, p. 45-58, 2000.

HADADIN, N. A. Rainfall Intensity-Duration-Frequency Relationship in the Mujib Basin in Jordan. Journal of Applied Sciences, v. 5, p. 1777-1784, 2005. http://dx.doi.org/10.3923/jas.2005.1777.1784

ITCF - INSTITUTO DE TERRAS, CARTOGRAFIA E FLORESTAS. Atlas do Estado do Paraná. Curitiba, 1987.

KOTHYARI, U. C.; GARDE, R. J. Rainfall intensity-duration-frequency formula for India. Journal of Hydraulic Engineering, v. 118, n. 2, p. 323-336, 1992. http://dx.doi.org/10.1061/(ASCE)0733-9429(1992)118:2(323)

LEVINE, D. M.; STEPHAN, D.; KREHBIEL, T. C.; BERENSON, M. L. Estatística - Teoria e Aplicações. Rio de Janeiro: Livros Técnicos e Científicos, 2005. 
NALI, J. O.; REIS, J. A. T.; REIS, A. O. P.; SILVA, F. G. B. Avaliação da resposta de diferentes de equações de chuvas intensas obtidas para as bacias hidrográficas dos rios Jucu e Santa Maria da Vitória, ES. In: SIMPÓSIO BRASILEIRO DE RECURSOS HÍDRICOS, 12. 2007. Anais... São Paulo: ABRH, 2007. 1 CD-ROM.

OCCHIPINTI, A. G.; SANTOS, P. M. Relação entre as precipitações de "Um Dia" e "24 Horas" na cidade de São Paulo. São Paulo: Instituto Astronômico e Geofísico - USP, 1966.

OLIVEIRA, L. F. C.; ANTONINI, J. C. A., GRIEBELER, N. P. Estimativas de chuvas intensas para o estado de Goiás. Revista de Engenharia Agrícola, v. 28, n. 1, p. 22-33, 2008.

OLIVEIRA, L. F. C.; CORTÊS, F. C.; BARBOSA, F. O. A.; ROMÃO, P. A.; CARVALHO D. F. Estimativa das equações de chuvas intensas para algumas localidades no Estado de Goiás pelo método da desagregação de chuvas. Pesquisa Agropecuária Tropical, v. 30, n. 1, p. 23-27, 2000.

OLIVEIRA, L. F. C.; CORTÊS, F. C., WEHR, T. R. Intensidade-Duração-Frequência de chuvas intensas para localidades no estado de Goiás e Distrito Federal. Pesquisa Agropecuária Tropical, v. 35, n. 1, p. 13-18, 2005.

PFAFSTETTER. O. Chuvas Intensas no Brasil. Rio de Janeiro: DNOS, 1957.

REICH, B. M. Short-duration rainfall-intensity estimates and other design aids for regions of sparse data. Journal of Hydrology, v. 1, n. 1, p. 3-28, 1963. http://dx.doi.org/10.1016/0022-1694(63)90029-5

RIGHETTO, A. M. Hidrologia e Recursos Hídricos. São Calos: EESC/USP, 1998.

RODRIGUES, J. O.; ANDRADE, E. M.; OLIVEIRA, T. S.; LOBATO, F. A. O. Equações de intensidade-duração-frequência de chuvas para localidades de fortaleza e pentecoste. Scientia Agraria, v. 9, n. 4, p. 511-519, 2008.

SILVA, D. D.; FILHO, R. R. G.; PRUSKI, F. F.; PEREIRA, S. B.; NOVAES, L. F. Chuvas intensas no estado da Bahia. Revista Brasileira de Engenharia Agrícola e Ambiental, v. 6, n. 2, p. 362-367, 2000.

SINGH, V. P.; ZHANG, L. IDF curves using the Frank Archimedean copula. Journal of Hydrologic Engineering, v. 12, n. 6, p. 651-662, 2007. http://dx.doi.org/10.1061/(ASCE)1084-0699(2007)12:6(651) 


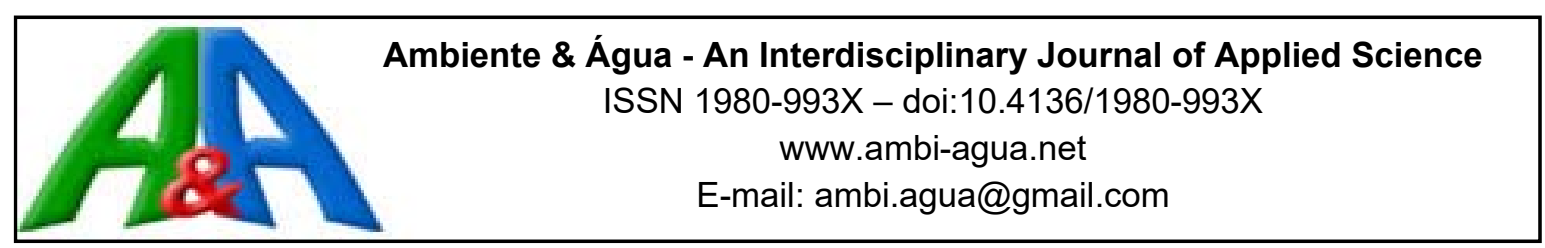

\title{
Simulação numérica da dispersão do sulfeto de hidrogênio emitido por um reator UASB para tratamento de esgoto doméstico
}

\author{
doi:10.4136/ambi-agua.1841 \\ Received: 15 Jan. 2016; Accepted: 16 Jan. 2017 \\ Matheus Ribeiro Augusto ${ }^{1 *}$; Herlane Costa Calheiros²; \\ Vanessa Silveira Barreto Carvalho ${ }^{2}$ \\ ${ }^{1}$ Universidade de São Paulo, Escola Politécnica, (USP), São Paulo, SP, Brasil \\ Departamento de Engenharia Hidráulica e Ambiental \\ ${ }^{2}$ Universidade Federal de Itajubá (UNIFEI), Itajubá, MG, Brasil \\ Instituto de Recursos Naturais (IRN) \\ *Autor correspondente: e-mail: matheus.augusto047@gmail.com, \\ h2c@unifei.edu.br, vanessa.silveira@gmail.com
}

\section{RESUMO}

O tratamento anaeróbio de águas residuárias pode produzir elevadas concentrações de sulfeto de hidrogênio $\left(\mathrm{H}_{2} \mathrm{~S}\right)$, substância que apresenta forte odor, além de ser corrosivo e tóxico. Alguns dos métodos existentes para o controle do $\mathrm{H}_{2} \mathrm{~S}$ demandam elevado custo de implantação e operação e, portanto, não são adotados. Dessa forma, o planejamento adequado da instalação de uma estação de tratamento de esgoto (ETE) pode auxiliar na prevenção do impacto e de possíveis problemas com a população. Modelos matemáticos de dispersão atmosférica podem ser aplicados previamente para verificar o alcance do poluente e, portanto, subsidiar a definição do local mais adequado para instalação de uma ETE. Dessa forma, o objetivo deste trabalho foi analisar a dispersão atmosférica do $\mathrm{H}_{2} \mathrm{~S}$ proveniente de uma unidade anaeróbia de tratamento de águas residuárias localizada em um bairro rural do município de Itajubá, MG, Brasil. Para isso, foi aplicado o modelo gaussiano AERMOD utilizando-se dados reais da taxa de emissão do poluente no reator anaeróbio, além de informações meteorológicas e geofísicas (relevo e uso do solo). Como resultado das simulações verificou-se que a pluma de odor foi orientada pelo relevo e se concentrou nas proximidades da unidade de tratamento, limitando o impacto ao bairro rural. $\mathrm{O}$ vento também exerceu influência direta na orientação do $\mathrm{H}_{2} \mathrm{~S}$ para o período 1 . No entanto, para o período 2, não se verificou a mesma tendência. Dessa maneira, constatou-se que a análise de características meteorológicas (principalmente a direção predominante dos ventos) e geofísicas da região e a aplicação de modelos matemáticos podem auxiliar na escolha do local mais adequado para a instalação de uma ETE.

Palavras-chave: AERMOD, dispersão atmosférica, $\mathrm{H}_{2} \mathrm{~S}$, odor.

\section{Numerical simulation of the dispersion of hydrogen sulphide emitted by a UASB reactor for domestic sewage treatment}

\section{ABSTRACT}

The anaerobic treatment of wastewaters can produce a large concentration of hydrogen sulfide $\left(\mathrm{H}_{2} \mathrm{~S}\right)$, an odorant, toxic and corrosive substance. Control methods for eliminating $\mathrm{H}_{2} \mathrm{~S}$ often require high implementation and operation costs and therefore are not used. Thus, suitable 
planning of wastewater treatment plant (WWTP) installation can help to prevent adverse impacts and potential problems for nearby communities. Mathematical models of atmospheric dispersion can be applied to verify the pollutant range and support the choice of the most appropriate place for installing a WWTP. Thus, the aim of this work was to analyze the dispersion of $\mathrm{H}_{2} \mathrm{~S}$ from an anaerobic unit of wastewater treatment located in a rural district of the city of Itajubá (MG), Brasil. For this, a Gaussian model AERMOD was applied using actual pollutant emission rate data in the UASB reactor, as well as meteorological and geophysical information (topography and land use). Using simulations, it was found that the odor plume was oriented by relief and concentrated near the treatment unit, limiting the impact to the rural district. The wind had also direct influence on the direction of $\mathrm{H}_{2} \mathrm{~S}$ for the first period. However, the same trend was not found in the second study period. Thus, it was found that the analysis of meteorological characteristics (especially the predominant wind direction) and geophysics in the region and the application of mathematical models can assist in choosing the most suitable location for the installation of a WWTP.

Keywords: AERMOD, atmospheric dispersion, $\mathrm{H}_{2} \mathrm{~S}$, odor.

\section{INTRODUÇÃO}

O tratamento anaeróbio de águas residuárias utilizando-se reatores UASB (Upflow Anaerobic Sludge Blanket) é amplamente aplicado no Brasil e no mundo, principalmente devido às vantagens econômicas em relação aos sistemas aeróbios. No entanto, a degradação anaeróbia da matéria orgânica pode seguir uma rota metabólica sulfetogênica, na qual o sulfato $\left(\mathrm{SO}_{4}{ }^{2-}\right)$ é reduzido, pela ação das bactérias redutoras de sulfato (BRS), a sulfeto de hidrogênio $\left(\mathrm{H}_{2} \mathrm{~S}\right)$, gás que pode se desprender da fase líquida para a atmosfera (Chernicharo, 2007).

$\mathrm{O} \mathrm{H}_{2} \mathrm{~S}$ é tóxico e apresenta odor agressivo, podendo impactar negativamente as pessoas que vivem nos arredores das estações de tratamento de esgoto (ETEs) (Chernicharo, 2007; Santos et al., 2012).

Segundo a Organização Mundial de Saúde (World Health Organization), a presença do $\mathrm{H}_{2} \mathrm{~S}$ na atmosfera em concentrações superiores a $15 \mathrm{mg} \mathrm{m}^{-3}$ pode causar efeitos adversos à saúde humana, desde dores de cabeça, náusea e simples problemas respiratórios até a morte, para valores superiores a $1400 \mathrm{mg} \mathrm{m}^{-3}$ (WHO, 2000). Em relação ao odor, o $\mathrm{H}_{2} \mathrm{~S}$ pode ser percebido e até mesmo causar incômodo em baixas concentrações, da ordem de $0,655 \mu \mathrm{g} \mathrm{m}^{-3}$ (equivalente a $0,00047 \mathrm{ppm}$, a $25^{\circ} \mathrm{C}$ e 1 atm de pressão) (Tchobanoglous et al., 2003; Santos et al., 2012). Dessa forma, é fundamental realizar o monitoramento e o controle adequado do contaminante para minimizar o impacto à vizinhança das ETEs.

Modelos de dispersão atmosférica podem auxiliar e orientar o controle/monitoramento do $\mathrm{H}_{2} \mathrm{~S}$, uma vez que é possível verificar o comportamento do poluente na atmosfera e, portanto, a abrangência do impacto ambiental, a partir de cenários pré-estabelecidos. É possível ainda analisar a influência da estabilidade atmosférica e outras condições meteorológicas, além das características geofísicas (relevo e uso do solo) na dispersão do poluente (Sironi et al., 2010; Latos et al., 2011; O’Shaughnessy e Altmaier, 2011; Melo et al., 2012; Olafsdottir et al., 2014; Abdul-Wahab et al., 2014).

Latos et al. (2011) e Abdul-Wahab et al. (2014) verificaram, em modelos de dispersão AERMOD e CALPUFF, respectivamente, que quanto maior a estabilidade atmosférica, maiores são as concentrações de $\mathrm{H}_{2} \mathrm{~S}$ nas proximidades da fonte, visto que a turbulência (mecânica e convectiva) e, consequentemente, o transporte de poluentes são dificultados nessa situação. Abdul-Wahab et al. (2014) ainda verificaram que locais com características climáticas e geofísicas distintas apresentaram diferentes tendências de dispersão. 
Olafsdottir et al. (2014), utilizando o modelo AERMOD, observaram a influência significativa do relevo na dispersão do poluente, visto que cadeias montanhosas tenderam a reter e orientar a pluma de dispersão, principalmente durante condições de grande estabilidade atmosférica.

Dessa maneira, a caracterização do local do empreendimento quanto à topografia, uso do solo e clima, pode auxiliar no gerenciamento do poluente desde a etapa de concepção até a operação da unidade de tratamento de águas residuárias.

Nesse contexto, o objetivo deste trabalho é analisar a dispersão atmosférica do $\mathrm{H}_{2} \mathrm{~S}$ emitido de um reator UASB localizado em um bairro rural do município de Itajubá, MG, Brasil.

\section{MATERIAL E MÉTODOS}

\subsection{Local de estudo}

O estudo foi realizado para uma ETE localizada em um bairro rural do município de Itajubá, MG, Brasil. A ETE é constituída por uma unidade de gradeamento, um reator UASB fechado de seção circular com uma única saída de biogás para a atmosfera e um filtro biológico anaeróbio totalmente fechado. O sistema foi projetado para tratar apenas o esgoto doméstico do bairro, cujo volume médio diário não ultrapassa $24 \mathrm{~m}^{3}$ (vazão de aproximadamente $0,28 \mathrm{~L} \mathrm{~s}^{-1}$ ).

A simulação de dispersão atmosférica foi realizada considerando o reator UASB como fonte principal de emissão do $\mathrm{H}_{2} \mathrm{~S}$ (na saída do biogás para a atmosfera), como verificado em estudos preliminares realizados por Meira (2014) e Presotto (2014). A unidade apresenta as seguintes dimensões: 2,10 metros de diâmetro interno; 2,80 metros de diâmetro externo e 5,80 metros de profundidade. $\mathrm{O}$ filtro anaeróbio também exibe grande potencial de emissão de $\mathrm{H}_{2} \mathrm{~S}$, no entanto, a unidade encontra-se totalmente fechada e apresenta a saída do efluente tratado "afogada" (submersa no corpo receptor).

\subsection{Caracterização do uso do solo}

Foi realizada a classificação do uso do solo do local de estudo, visto que as características de superfície (Albedo, Rugosidade e Razão de Bowen), importantes variáveis do processo de dispersão atmosférica, variam conforme a cobertura do terreno.

Foi analisado um raio de $4 \mathrm{~km}$ ao redor da fonte (ETE). Realizou-se uma classificação supervisionada, através do Sistema de Informação Geográfica (SIG) SPRING, versão 5.2.7, desenvolvido pelo Instituto Nacional de Pesquisas Espaciais (INPE). Consideraram-se as seguintes classes de uso do solo: floresta, pastagem e urbano.

\subsection{Simulação da dispersão atmosférica}

A simulação da dispersão atmosférica do poluente foi realizada através do modelo gaussiano AERMOD, proposto pela USEPA (2004) e desenvolvido pela AERMIC (AMS/EPA Regulatory Model Improvement Committee). O modelo regulatório é divido em um módulo principal e dois pré-processadores: AERMET e AERMAP. O AERMET processa as informações meteorológicas e o AERMAP os dados topográficos. O módulo principal AERMOD realiza os cálculos da dispersão atmosférica, utilizando o produto dos dois pré-processadores. O modelo é disponibilizado na página da U.S. Environmental Protection Agency - USEPA (http://www3.epa.gov/ttn/scram/dispersion_prefrec.htm\#aermod).

\subsubsection{Dados meteorológicos: AERMET}

Os dados meteorológicos, horários de temperatura, velocidade e direção do vento, essenciais ao pré-processador AERMET, foram obtidos através da base de dados do Centro de Estudos e Previsão de Tempo e Clima de Minas Gerais (CEPreMG) da Universidade Federal de Itajubá (UNIFEI), localizada a aproximadamente $7 \mathrm{~km}$ da ETE. 
Os demais dados meteorológicos de superfície - nebulosidade (cobertura de nuvens) e altura de base das nuvens - são referentes ao Aeroporto de São José dos Campos. Esses dados estão disponibilizados na base de dados da Rede de Meteorologia da Aeronáutica - REDEMET (www.redemet.aer.mil.br).

As características de superfície, também dados essenciais a essa etapa, foram estimados em função do uso do solo no local, a partir dos valores propostos por USEPA (2013).

\subsubsection{Dados do terreno: AERMAP}

Nessa etapa é definido o domínio da modelagem através de uma grade de receptores. Foi definida uma grade cartesiana $(81$ x 81) com resolução espacial de 100 metros, totalizando 6561 receptores e cobrindo um raio de aproximadamente $4 \mathrm{~km}$ ao redor da fonte.

O modelo digital de elevação (MDE), dado essencial para extração da topografia, foi obtido da "United States Geological Survey" (USGS) (https://earthexplorer.usgs.gov/). Optou-se pelo modelo ASTGTM GDEM devido a sua elevada resolução espacial (30 metros).

\subsubsection{Módulo principal: AERMOD}

No módulo principal é realizada a simulação da dispersão atmosférica do poluente. Além dos produtos dos pré-processadores AERMET e AERMAP, a taxa de emissão do composto em $\mathrm{g} \mathrm{s}^{-1}$ é uma informação essencial para o módulo AERMOD.

A taxa de emissão do $\mathrm{H}_{2} \mathrm{~S}$ foi obtida a partir de medições em campo realizadas por Presotto (2014) durante os meses de novembro e dezembro de 2013 (período 1), e Meira (2014) durante o mês de janeiro de 2014 (período 2). Meira (2014) e Presotto (2014) instalaram um compressor aspirador à vácuo no duto de saída do gás do reator UASB e estabeleceram uma vazão média de sucção de aproximadamente $15 \mathrm{~L} \mathrm{~min}^{-1}$. O biogás coletado era borbulhado em solução de zinco alcalina para extração do sulfeto de hidrogênio. O composto era analisado posteriormente por iodometria. As taxas de emissão foram obtidas através do produto entre a vazão de sucção e as concentrações de saída do $\mathrm{H}_{2} \mathrm{~S}$.

No período 1, as taxas médias diárias de emissão de $\mathrm{H}_{2} \mathrm{~S}$ variaram entre 4,2 e 32,8 $\mu \mathrm{g} \mathrm{s}^{-1}$, com um valor médio de $16,4 \mu \mathrm{g} \mathrm{s}^{-1}$ para o período. No período 2 , os valores variaram entre 2,6 e $59,9 \mu \mathrm{g} \mathrm{s}^{-1}$, com um valor médio de $22,5 \mu \mathrm{g} \mathrm{s}^{-1}$.

As simulações de dispersão atmosférica do $\mathrm{H}_{2} \mathrm{~S}$ foram realizadas para os mesmos períodos das medições de campo de Presotto (2014) e Meira (2014).

Foram geradas figuras, para cada um dos períodos, do pior cenário (maior concentração média horária do poluente) e do cenário médio (concentração média do poluente durante o período). Os resultados das simulações foram trabalhados no SIG QGIS, versão 2.12, para criação das figuras de distribuição espacial do $\mathrm{H}_{2} \mathrm{~S}$.

\subsubsection{Influência de variáveis meteorológicas e geofísicas}

Foi verificada a influência da direção predominante dos ventos na orientação da pluma de $\mathrm{H}_{2} \mathrm{~S}$. Para isso, foram criadas rosas dos ventos para os dois períodos estudados, através dos dados horário de velocidade e direção do vento, utilizando-se o software WRPLOT "view", versão 7.0.0, desenvolvido pela "Lakes Environmental”.

Para verificar a possível influência do relevo na dispersão do poluente, foi criado um mapa hipsométrico, a partir do mesmo MDE utilizado no pré-processador AERMAP.

\section{RESULTADOS E DISCUSSÃO}

\subsection{Uso do solo e características de superfície}

A caracterização do local de estudo quanto ao uso do solo está apresentada na Figura 1. 


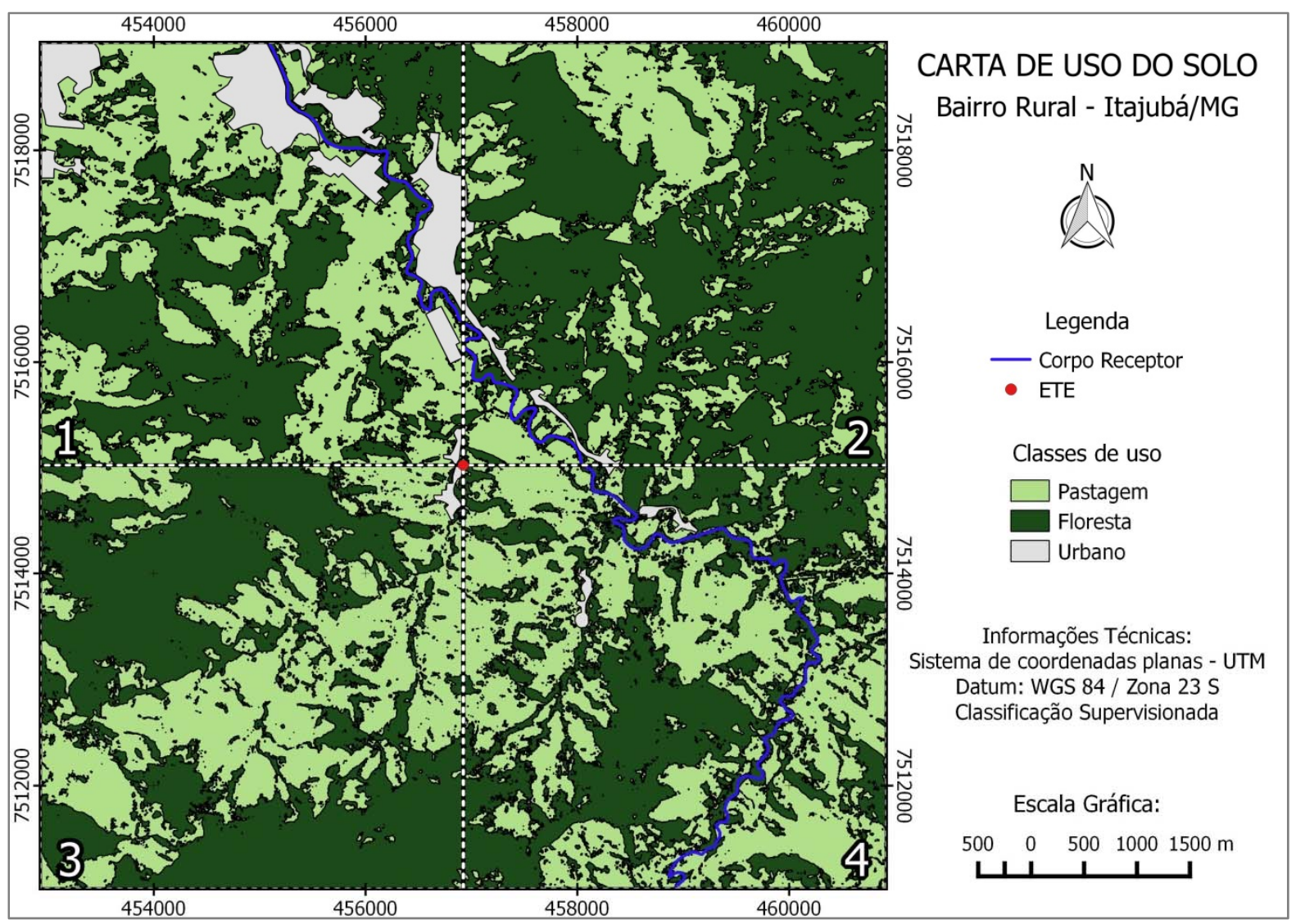

Figura 1. Carta do uso do solo da área de estudo.

O local de estudo foi dividido em quatro quadrantes, uma vez que a distribuição das diferentes classes de uso do solo (floresta, pastagem e urbano) não é homogênea no domínio da modelagem. Evidencia-se a predominância de pastagem/solo exposto e floresta (mata) considerando todo o domínio da modelagem. Somente no quadrante 1, à noroeste da ETE, existe uma mancha urbana significativa, correspondente a $11,7 \%$ do total do quadrante.

Nos quadrantes 2 e 3 ocorre a predominância de floresta (66,4 e 55,2 \%, respectivamente), enquanto nos quadrantes 1 e 4 a pastagem apresenta maior área $(47,6$ e 56,0 \%, respectivamente). No entanto, existe um relativo equilíbrio entre essas duas classes de uso do solo.

A partir da classificação quanto ao uso do solo, determinaram-se as características de superfície resultantes para cada quadrante, considerando-se os valores propostos pela USEPA (2013) para cada classe de uso e as estações meteorológicas primavera (referente ao mês de novembro de 2013) e verão (referente aos meses dezembro de 2013 e janeiro de 2014). Essas informações foram utilizadas como entrada do pré-processador meteorológico AERMET, para simulação de dispersão atmosférica.

\subsection{Distribuição espacial do $\mathrm{H}_{2} \mathrm{~S}$}

\subsubsection{Maior concentração média horária (pior cenário)}

As simulações de dispersão do $\mathrm{H}_{2} \mathrm{~S}$ foram realizadas através do modelo AERMOD, para os meses de novembro e dezembro de 2013 (período 1) e janeiro de 2014 (período 2), a partir de dados de emissão do poluente medidos em campo. A Figura 2 apresenta a distribuição espacial das concentrações de $\mathrm{H}_{2} \mathrm{~S}$ na atmosfera, considerando-se a maior concentração média horária para os dois períodos analisados. 


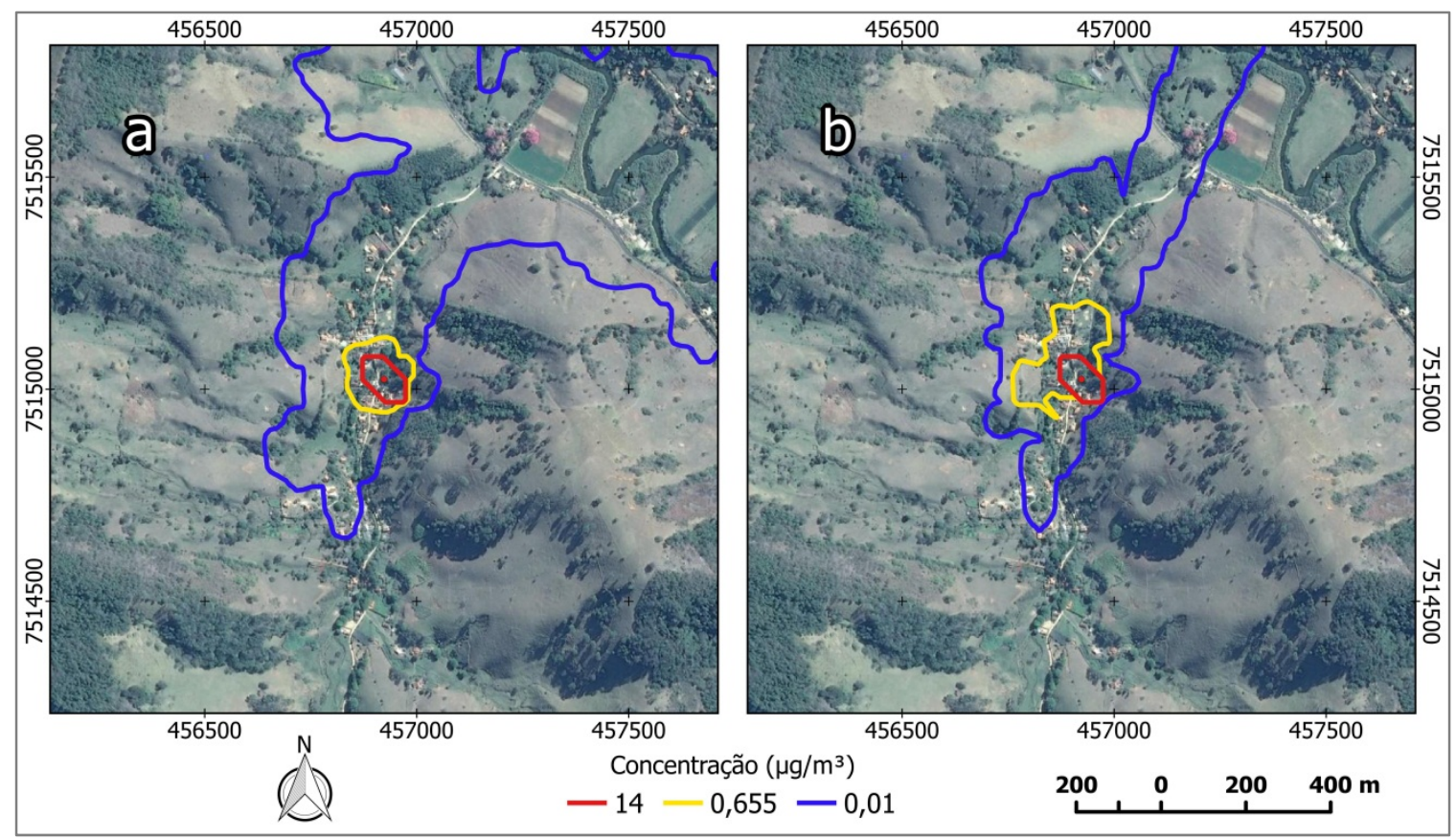

Figura 2. Distribuição espacial das maiores concentrações horárias de $\mathrm{H}_{2} \mathrm{~S}$ na atmosfera para novembro e dezembro de 2013 (período 1) (a) e janeiro de 2014 (período 2) (b).

As isolinhas de concentração estão em $\mu \mathrm{g} \mathrm{m}^{-3}\left(1,0 \mu \mathrm{g} \mathrm{m}^{-3}\right.$ é equivalente a aproximadamente $0,72 \mathrm{ppb}$ ). A isolinha de $0,655 \mu \mathrm{g} \mathrm{m}^{-3}$ (pluma de odor) representa o limite olfativo $(0,47 \mathrm{ppb})$, enquanto a isolinha de $14 \mu \mathrm{g} \mathrm{m}^{-3}$ representa a região de maior potencial de incômodo em relação ao odor (Tchobanoglous et al., 2003). As demais isolinhas, com concentrações inferiores, foram plotadas apenas para indicar a direção resultante de deslocamento da pluma.

Para o pior cenário referente ao período 1 (Figura 2(a)), o raio médio da isolinha de concentração na faixa do limite olfativo $\left(0,655 \mu \mathrm{g} \mathrm{m}^{-3}\right)$ foi de aproximadamente $100 \mathrm{~m}$. Já para o período 2 (Figura 2b), a pluma de odor apresentou maior abrangência, alcançando aproximadamente $200 \mathrm{~m}$ na direção norte $\left(0^{\circ}\right)$. Ainda assim, o impacto relacionado à emissão do gás odorante para os períodos analisados foi bastante restrito às proximidades da ETE.

A isolinha de concentração de $14 \mu \mathrm{g} \mathrm{m}^{-3}$ apresentou um alcance médio de $36 \mathrm{~m}$ ao redor da fonte para ambos os períodos.

Observa-se ainda uma grande tendência de dispersão do poluente para região localizada a norte-nordeste $\left(22,5^{\circ}\right)$ da fonte.

Através das simulações de dispersão, não foram verificadas, ao redor da fonte, concentrações superiores ao valor de referência para exposição aguda $\left(15.10^{3} \mu \mathrm{g} \mathrm{m}^{-3}\right)$ proposto por WHO (2000), acima do qual os riscos à saúde humana aumentam. No entanto, segundo um levantamento realizado por Presotto (2014) no bairro onde está localizada a ETE, grande parte dos moradores alega sentir dores de cabeça em alguns momentos do dia, decorrentes da exposição ao $\mathrm{H}_{2} \mathrm{~S}$. Outros sintomas relacionados à exposição ao poluente, tais como tontura, estresse e náusea também foram notados entre os entrevistados. Dessa maneira, os resultados das simulações não eliminam a necessidade de realizar o monitoramento do $\mathrm{H}_{2} \mathrm{~S}$ no local. As taxas de emissão do poluente podem ser maiores do que os valores obtidos experimentalmente e o verdadeiro impacto aos moradores pode estar sendo subestimado.

Presotto (2014) ainda verificou a opinião dos moradores quanto à intensidade do odor emitido pela ETE. Os resultados da pesquisa indicaram que o odor é mais intenso na região central do bairro, mais próxima à ETE, seguida pela região ao norte da unidade. A região sul do bairro apresentou menor porcentagem de reclamações quanto ao odor emitido no tratamento. 
Essas tendências coincidiram com os resultados das simulações de dispersão do sulfeto de hidrogênio realizadas para os períodos 1 e 2 (Figura 2).

\subsubsection{Concentração média (cenário médio)}

A Figura 3 apresenta a distribuição espacial das concentrações de $\mathrm{H}_{2} \mathrm{~S}$ na atmosfera próxima a ETE e corresponde à média do período (cenário médio).

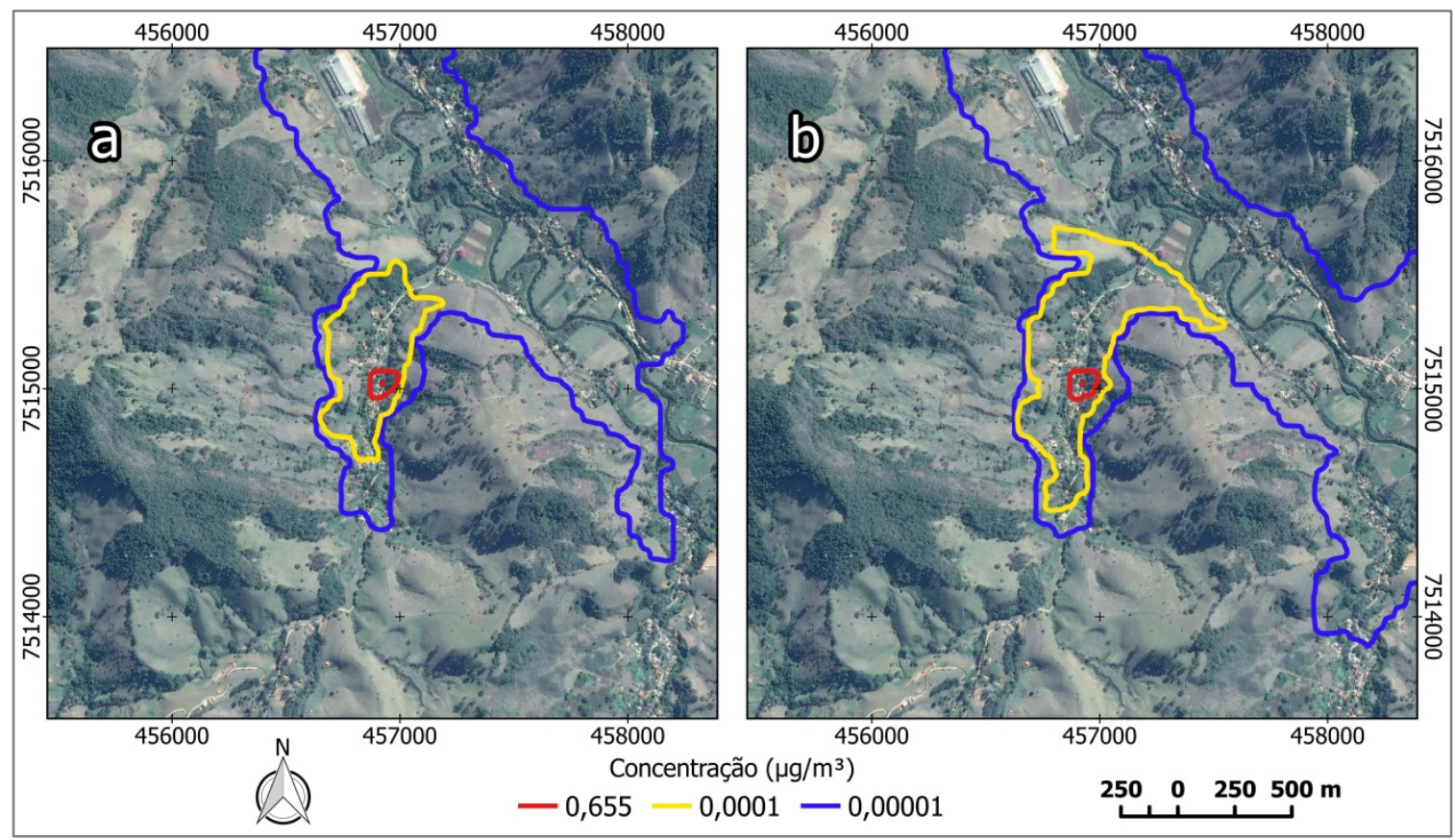

Figura 3. Distribuição espacial das concentrações médias de $\mathrm{H}_{2} \mathrm{~S}$ na atmosfera para novembro e dezembro de 2013 (período 1) (a) e janeiro de 2014 (período 2) (b).

A isolinha de concentração de $0,655 \mu \mathrm{g} \mathrm{m}^{-3}$, considerando-se o cenário médio de dispersão para o período 1 (Figura 3a), apresentou um alcance médio de $60 \mathrm{~m}$ ao redor da fonte. Já para o período 2 (Figura $3 \mathrm{~b}$ ), o alcance médio desta foi ligeiramente superior: aproximadamente $65 \mathrm{~m}$. Dessa forma, somente as residências vizinhas à fonte seriam possivelmente impactadas na maior parte do tempo pelo odor do $\mathrm{H}_{2} \mathrm{~S}$, considerando-se ambos os períodos.

Não foram determinados, para o cenário médio de ambos os períodos, locais fora dos limites da ETE que apresentaram concentrações superiores aos valores de referência para exposição crônica $\left(150 \mu \mathrm{g} \mathrm{m}^{-3}\right)$ e aguda $\left(14.10^{3} \mu \mathrm{g} \mathrm{m}^{-3}\right)$, propostos por WHO (2000). No entanto, esses resultados são preditos e, portanto, não eliminam a necessidade de monitoramento do poluente no local de estudo para verificar os reais riscos toxicológicos.

Verifica-se, para ambos os períodos, uma tendência de dispersão do poluente muito semelhante, orientada para direção norte-nordeste $\left(22,5^{\circ}\right)$ até a margem do manancial principal, a partir da qual a pluma assume novas direções de deslocamento: noroeste $\left(315^{\circ}\right)$ e sudeste $\left(135^{\circ}\right)$.

\subsubsection{Distribuição dos ventos}

As rosas dos ventos para Itajubá, MG, Brasil, referentes aos períodos 1 e 2 (Figura 4) foram plotadas com intuito de se verificar a influência da direção predominante dos ventos na orientação da pluma de sulfeto de hidrogênio. 


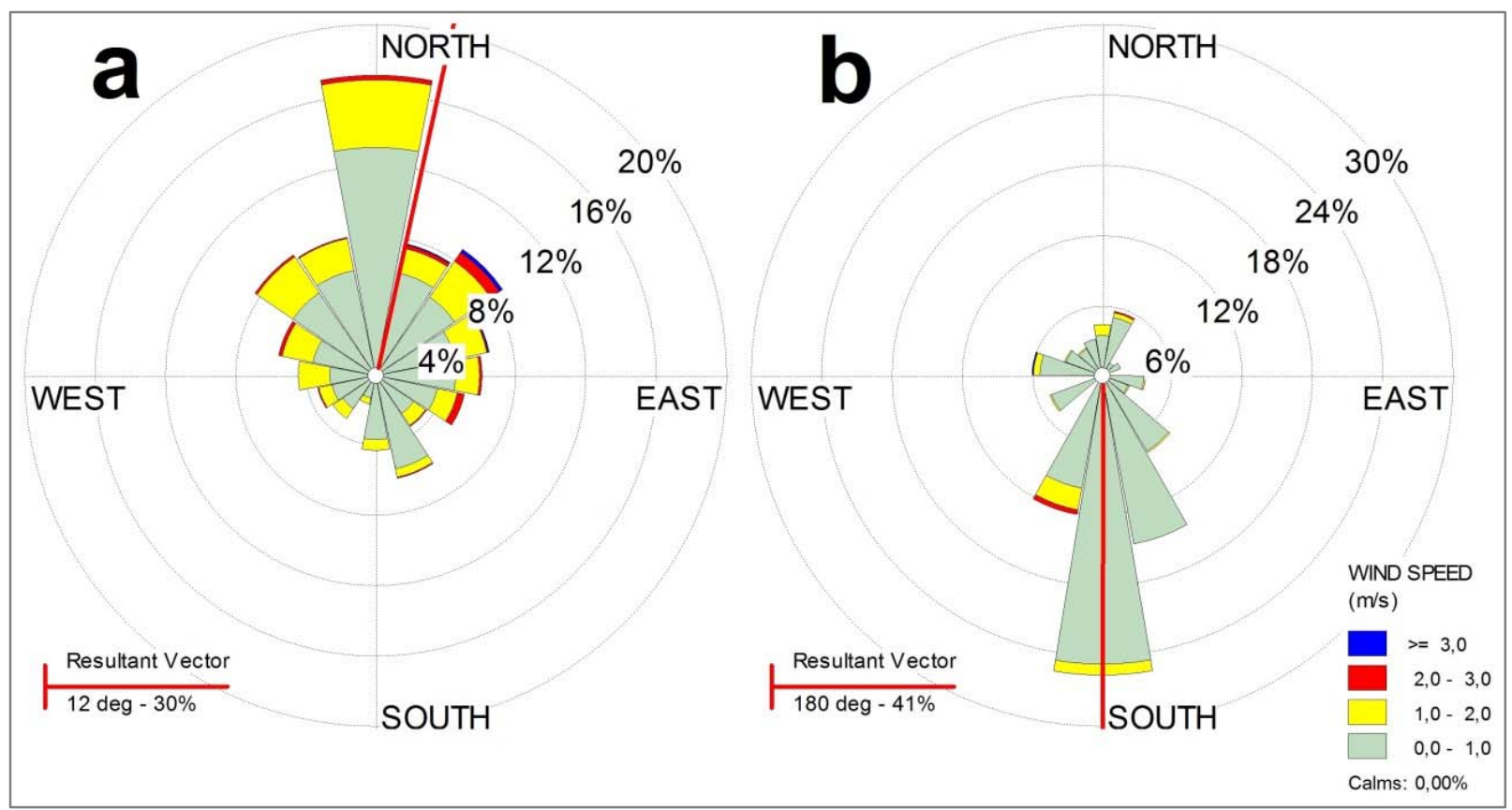

Figura 4. Rosa dos ventos (sentido do fluxo) para o município de Itajubá, MG, Brasil para novembro e dezembro de 2013 (período 1) (a) e janeiro de 2014 (período 2) (b).

No período 1 (Figura 4a), os ventos de sul-sudoeste, direcionados para norte-nordeste (vetor resultante: aproximadamente $12^{\circ}$ ), ocorreram com maior frequência. A dispersão do poluente para o cenário médio (Figura 3a) apresentou a mesma direção resultante de deslocamento, ou seja, possivelmente a forçante vento apresentou influência direta na orientação da pluma de odor. Outros trabalhos que analisaram a dispersão do sulfeto de hidrogênio também constataram a influência direta do vento na orientação e deslocamento do poluente (O'Shaughnessy e Altmaier, 2011; Olafsdottir e Gardarsson, 2013; Abdul-Wahab et al., 2014).

No período 2 (Figura $4 \mathrm{~b}$ ), os ventos de norte direcionados para o sul $\left(180^{\circ}\right)$ ocorreram com maior frequência. No entanto, analisando-se o deslocamento da pluma de $\mathrm{H}_{2} \mathrm{~S}$ para o cenário médio do mesmo período (Figura $4 \mathrm{~b}$ ), observa-se uma tendência de dispersão para região localizada a norte-nordeste $\left(22,5^{\circ}\right)$ da fonte. Dessa maneira, a dispersão do poluente, determinada através do modelo AERMOD, está seguindo direção oposta ao vetor resultante do vento (aproximadamente $180^{\circ}$ ). Sendo assim, possivelmente existem outras variáveis que apresentam peso mais significativo na orientação da pluma para esse caso específico, como o relevo.

\subsubsection{Composição do relevo}

Segundo Olafsdottir et al. (2014) e Abdul-Wahab et al. (2014), o relevo pode influenciar significativamente na orientação e deslocamento da pluma. Dessa forma, realizou-se a sobreposição da pluma de concentração de $\mathrm{H}_{2} \mathrm{~S}$ (isolinha de $0,00001 \mu \mathrm{g} \mathrm{m}^{-3}$, conforme Figura 3b) sobre a carta hipsométrica do local de estudo (Figura 5), considerando o cenário médio do período 2 (janeiro de 2014), no qual o alcance horizontal do poluente foi mais significativo.

Observa-se que a ETE, fonte de emissão do $\mathrm{H}_{2} \mathrm{~S}$, está localizada em uma região de fundo de vale, ou seja, o local de estudo apresenta relevo irregular que pode influenciar significativamente na orientação do poluente. 


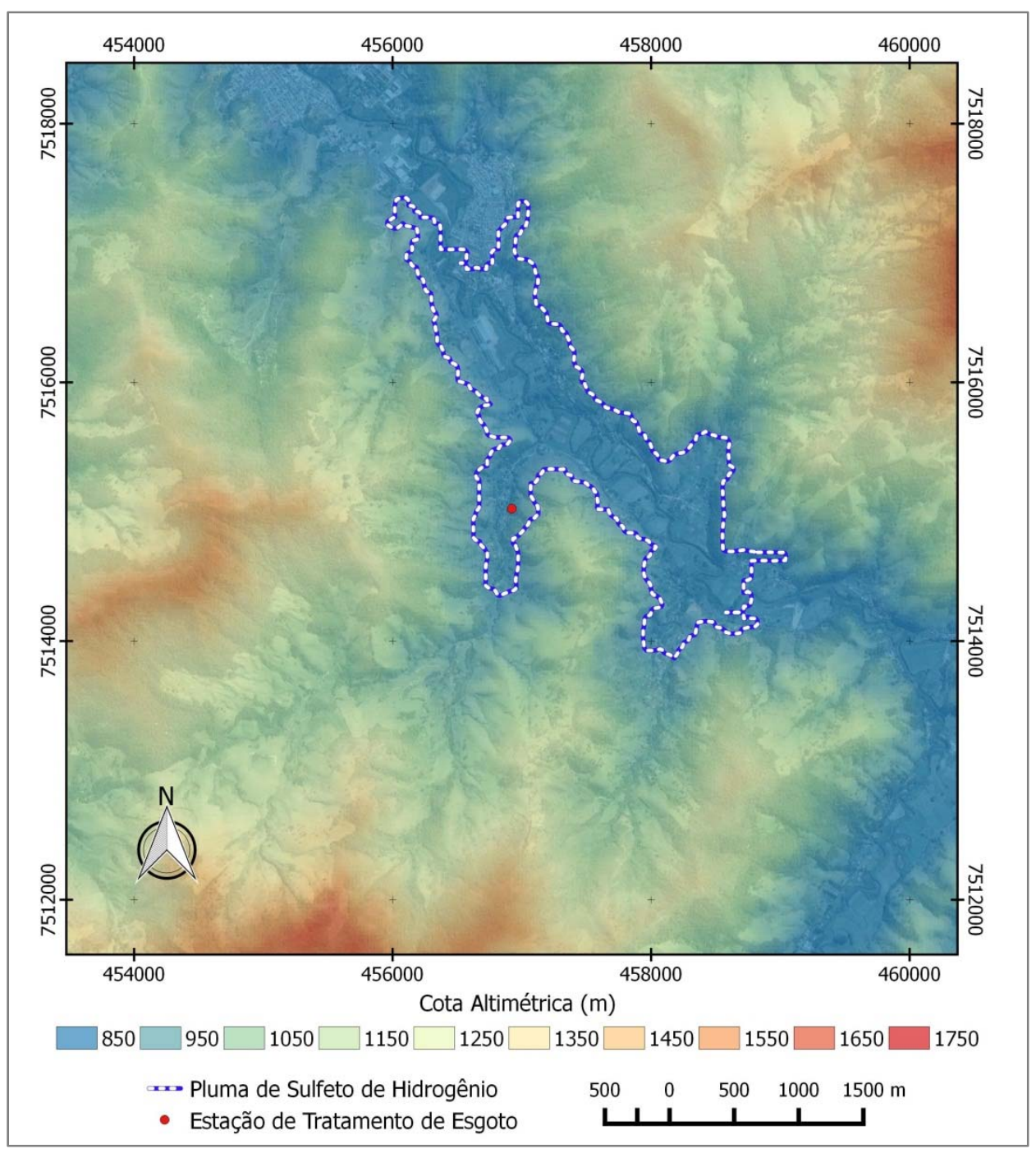

Figura 5. Influência do relevo na dispersão de $\mathrm{H}_{2} \mathrm{~S}$ em janeiro de 2014 (período 2).

Verifica-se uma relação muito forte entre o deslocamento da pluma de $\mathrm{H}_{2} \mathrm{~S}$ e o relevo do local de estudo. O poluente tende a se concentrar nas regiões mais baixas, com altitude aproximada de 850 metros. Dessa forma, os altos topográficos (divisor de águas) que delimitam a bacia hidrográfica onde se localiza a ETE atuam como barreiras que diminuem a efetividade da ação do vento e, portanto, dificultam a dispersão horizontal do poluente.

\section{CONCLUSÃO}

A partir dos resultados das simulações de dispersão do $\mathrm{H}_{2} \mathrm{~S}$ para ambos os períodos, verificou-se que a pluma de odor tende a se concentrar nas proximidades da ETE, impactando principalmente a população do bairro rural. Concentrações acima dos valores de referência para exposição crônica e aguda ao poluente não foram verificadas, considerando todo domínio da modelagem. No entanto, esses resultados são preditos e, portanto, não eliminam a necessidade de monitoramento do local de estudo e o controle adequado do contaminante. Portanto, recomenda-se que sejam realizadas mais medições da taxa de emissão de gás sulfídrico utilizando métodos mais precisos analiticamente. 
Verificou-se, para o cenário médio do período 1, que a pluma de odor seguiu a mesma direção do vetor resultante do vento. No entanto, para o período 2, não se observou essa relação. Em contrapartida, o relevo parece influenciar significativamente na dispersão do poluente, orientando a pluma de odor pelo fundo do vale, região entre cadeias montanhosas. Dessa maneira, os resultados desse trabalho permitem a visualização do problema e podem subsidiar e orientar a mitigação do impacto.

\section{AGRADECIMENTOS}

Agradecemos a CAPES pelo apoio financeiro à pesquisa e bolsa de mestrado, e ao GES - Grupo de Estudos em Saneamento da UNIFEI.

\section{REFERÊNCIAS}

ABDUL-WAHAB, S. A.; CHAN, K.; ELKAMEL, A.; AHMADI, L. Effects of meteorological conditions on the concentration and dispersion of an accidental release of $\mathrm{H}_{2} \mathrm{~S}$ in Canada. Atmospheric Environment, v. 82, p. 316-326, 2014. http://dx.doi.org/10.1016/j.atmosenv.2013.10.033

CHERnicharo, C. A. L. Princípio do tratamento biológico de águas residuárias: Reatores anaeróbios. 2. ed. Belo Horizonte: DESA/UFMG, 2007. 380 p.

LATOS, M.; KARAGEORGOS, P.; KALOGERAKIS, N.; LAZARIDIS, M. Dispersion of Odorous Gaseous Compounds Emitted from Wastewater Treatment Plants. Water, Air, \& Soil Pollution, v. 215, n. 1, p. 667-677, 2011. http://dx.doi.org/10.1007/s11270-0100508-8

MEIRA, L. H. R. Estudo da remoção de sulfeto de hidrogênio e amônia utilizando resíduos alcalinos da construção civil. 2014. Dissertação (Mestrado) - Universidade Federal de Itajubá, Itajubá, 2014.

MELO, A. M. V.; SANTOS, J. M.; MAVROIDIS, I.; REIS JR, N. C. Modelling of odour dispersion around a pig farm building complex using AERMOD and CALPUFF. Comparison with wind tunnel results. Building and Environment, v. 56, p. 8-20, 2012. http://dx.doi.org/10.1016/j.buildenv.2012.02.017

OLAFSDOTTIR, S.; GARDARSSON, S. M. Impacts of meteorological factors on hydrogen sulfide concentration downwind of geothermal power plants. Atmospheric Environment, v. 77, p. 185-192, 2013. http://dx.doi.org/10.1016/j.atmosenv. 2013.04.077

OLAFSDOTTIR, S.; GARDARSSON, S. M.; ANDRADOTTIR, H. O. Spatial distribution of hydrogen sulfide from two geothermal power plants in complex terrain. Atmospheric Environment, v. 82, p. 60-70, 2014. http://dx.doi.org/10.1016/j.atmosenv.2013.10.013

O'SHAUGHNESSY, P. T.; ALTMAIER, R. Use of AERMOD to determine a hydrogen sulfide emission factor for swine operations by inverse modeling. Atmospheric Environment, v. 45, n. 27, p. $4617-4625,2011$.

http://dx.doi.org/10.1016/j.atmosenv.2011.05.061

PRESOTTO, A. B. T. Estudo do controle de emissões de sulfeto de hidrogênio em sistema de tratamento anaeróbio de esgoto doméstico através de adsorção em carvão. 2014. Dissertação (Mestrado) - Universidade Federal de Itajubá, Itajubá, 2014. 
SANTOS, J. M.; KREIM, V.; JEAN-MICHEL, G.; REIS JR., N. C.; SÁ, L. M.; HORAN, N. J. An experimental determination of the $\mathrm{H}_{2} \mathrm{~S}$ overall mass transfer coeficient from quiescent surfaces at wastewater treatment plants. Atmospheric Environment, v. 60, p. 18-24, 2012. http://dx.doi.org/10.1016/j.atmosenv.2012.06.014

SIRONI, S.; CAPELLI, L.; CÉNTOLA, P.; DEL ROSSO, R.; PIERUCCI, S. Odour impact assessment by means of dynamic olfactometry, dispersion modeling and social participation. Atmospheric Environment, v. 44, n. 3, p. 354-360, 2010. http://dx.doi.org/10.1016/j.atmosenv.2009.10.029

TCHOBANOGLOUS, G.; BURTON, F. L.; STENSEL, H. D. Wastewater Engineering: treatment and reuse. 4. ed. New York: McGraw-Hill, 2003.

UNITED STATES. Environmental Protection Agency - USEPA. AERMOD: Description of Model Formulation. North Carolina: Research Triangle Park, 2004. 91 p.

UNITED STATES. Environmental Protection Agency - USEPA. AERSURFACE User's Guide. North Carolina: Research Triangle Park, 2013. 34 p.

WORLD HEALTH ORGANIZATION - WHO. Air Quality Guidelines for Europe. 2. ed. Copenhagen, 2000. 273 p. 


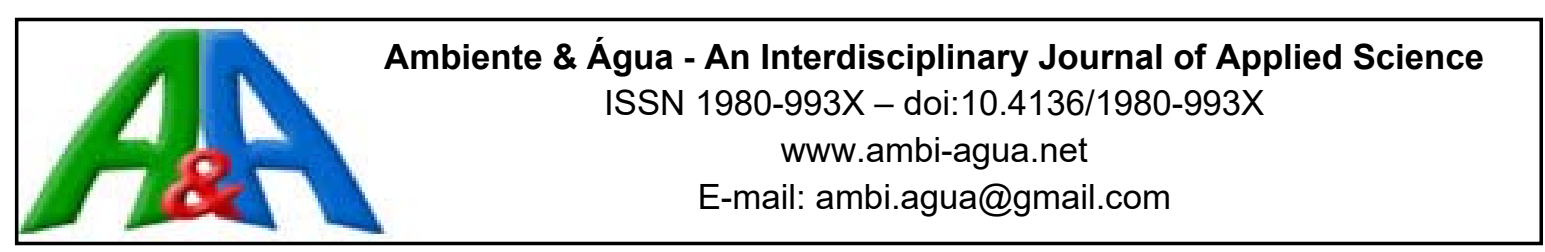

\title{
Calibração e validação do modelo SWAT para simulação hidrológica em uma bacia hidrográfica do litoral norte catarinense
}

\author{
doi:10.4136/ambi-agua.1951
}

Received: 1 Jun. 2016; Accepted: 18 Jan. 2017

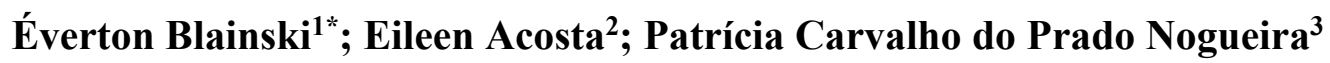 \\ ${ }^{1}$ Empresa de Pesquisa Agropecuária e Extensão Rural de SC (Epagri), Florianópolis, SC, Brasil \\ Centro de Informações de Recursos Ambientais e de Hidrometeorologia de SC (CIRAM) \\ ${ }^{2}$ The Nature Conservancy (TNC), Curitiba, PR, Brasil \\ Departamento de Ciências \\ ${ }^{3}$ Universidade Federal de Santa Catarina (UFSC), Florianópolis, SC, Brasil \\ Departamento de Engenharia Sanitária e Ambiental \\ *Autor correspondente: e-mail: evertonblainski@epagri.sc.gov.br, \\ eacosta@tnc.org, pati.cpn@hotmail.com
}

\section{RESUMO}

Os estudos hidrológicos em bacias experimentais têm sido realizados devido à necessidade de compreensão dos processos que controlam o movimento da água e sedimentos e seus impactos na disponibilidade e qualidade das águas. Os modelos hidrológicos são ferramentas com alto potencial para a simulação de cenários hipotéticos em diferentes escalas. Assim, o objetivo desse trabalho foi avaliar a eficácia do modelo SWAT para representação do fluxo hidrossedimentológico em uma bacia hidrográfica catarinense a partir dos procedimentos usuais de calibração e validação. O estudo foi realizado na bacia do rio Camboriú com uma área de $195 \mathrm{~km}^{2}$. Na análise do desempenho do modelo comparou-se os dados de vazão e sólidos transportados medidos e simulados na seção de controle. Os resultados obtidos atestaram a capacidade do SWAT para as simulações hidrossedimentológicas nessa bacia. Entretanto, foi preciso realizar a sua calibração para que as simulações atendessem os critérios de qualidade estabelecidos. Após a calibração, observou-se uma tendência de subestimativa das variáveis simuladas, porém, dentro de limites aceitáveis. Além disso, foi possível comprovar a eficiência do modelo para simulação das curvas de permanência das variáveis estudadas, caracterizando o SWAT como uma ferramenta importante na gestão de bacias hidrográficas como a do rio Camboriú.

Palavras-chave: gestão, transporte de sedimentos, vazão.

\section{Calibration and validation of the SWAT model for hydrologic simulation in a river basin located in the north coast of Santa Catarina}

\section{ABSTRACT}

Hydrological studies in experimental basins have been widely carried out in order to understand the processes that control the movement of water and sediment and their impact on the availability and quality of water. For this purpose, the hydrological models are used to simulate hypothetical scenarios at different spatial and temporal scales. The goal of this study was to evaluate the efficiency of the SWAT model to represent the hydrosedimentological flow 
in a Santa Catarina river basin by applying the usual procedures for calibration and validation. The study was conducted in the basin of the Camboriú River (BHRC), which has an area of 195 $\mathrm{km}^{2}$. For model performance analysis purposes, the flow and solids transported to the control section studied and the simulated data were compared. The results obtained have proven the efficiency of SWAT hydrosedimentological simulations in the BHRC. However, it was necessary to calibrate the model so that the simulations would comply with the established quality criteria. Though it occurred within acceptable limits, there was a tendency of underestimation of the simulated variables after calibration. Additionally, it was possible to verify the efficiency of the model for simulating the retention curves of the analyzed variables, thus emphasizing the SWAT as an important tool for river basin management such as the basin of Camboriú River.

Keywords: flow, management, sediment transport.

\section{INTRODUÇÃO}

Os estudos hidrológicos em bacias hidrográficas têm sido amplamente realizados a partir da necessidade de compreensão dos processos que controlam o movimento da água e sedimentos, e seus impactos sobre a disponibilidade e qualidade das águas. Nesse sentido, os modelos hidrológicos se consolidaram como ferramentas de representação desses processos com alto potencial para a simulação de cenários de mudanças climáticas, de alterações do uso do solo e de intervenções antrópicas em diferentes escalas espaciais e temporais.

A integração de modelos hidrológicos aos sistemas de informações geográficas proporcionou o desenvolvimento de ferramentas com maior embasamento físico, principalmente por possibilitar a representação dos parâmetros dos modelos de forma distribuída (Veith et al., 2010). Dessa integração, surgiu a possibilidade de ampliar o número de variáveis utilizadas para representar os fenômenos naturais dentro das bacias hidrográficas.

Apesar do grande número de variáveis incorporadas, os modelos hidrológicos são considerados representações simplificadas dos fenômenos naturais, e por mais sofisticados que sejam, ainda apresentam uma limitação relacionada à concepção e ao empirismo. De acordo com Muleta e Nicklow (2005), a qualidade dos resultados modelados depende da estrutura do modelo e dos algoritmos utilizados nas rotinas de cálculos, sendo que a qualidade dos dados de entrada para estimativa dos parâmetros representativos é de extrema importância.

Dentre as inúmeras opções de modelos hidrológicos disponíveis atualmente, o Soil and Water Assessment Tool (SWAT) tem sido bastante utilizado para simulações de vazões e sedimentos. O SWAT é um modelo semi-distribuído e contínuo no tempo, desenvolvido para simular o impacto da mudança do uso do solo em bacias hidrográficas de diferentes escalas (Arnold et al., 2012). Uma das facilidades para sua utilização é a interface acoplável aos principais softwares de geoprocessamento, o que possibilita a integração de bancos de dados, a elaboração e edição de cenários climatológicos e de uso das terras, e a análise e representação espacial das variáveis simuladas. No entanto, vale ressaltar que o SWAT é composto por um conjunto de equações que representam de forma simplificada o movimento da água, sedimentos e nutrientes em um sistema natural, razão pela qual deve ser calibrado e validado a partir de dados observados.

De acordo com Brighenti et al. (2016), é possível encontrar na literatura mais de 650 artigos publicados utilizando o SWAT como ferramenta de simulação. No Brasil, entre 1999 e 2013 foram publicados 102 trabalhos que relatam a utilização do SWAT para simulação do fluxo hidrossedimentológico. No entanto, um grande número dos trabalhos não descreve os processos de calibração e, principalmente, validação do modelo (Bressiani et al.,2015). Segundo os autores, a principal dificuldade para realizar a calibração e a validação de modelos é a ausência de séries de dados medidos nas bacias modeladas. 
A calibração do SWAT tem por objetivo principal minimizar os desvios entre as variáveis simuladas e medidas. Esse processo demanda amplo conhecimento em virtude do grande número de parâmetros não mensuráveis que precisam ser estimados (Veith et al., 2010). O processo de calibração automática facilita essa tarefa através da utilização de técnicas computacionais avançadas que possibilitam a alteração de parâmetros previamente selecionados dentro de uma faixa de valores estabelecida. A calibração pode ainda ser facilitada a partir da determinação do grau de influência dos parâmetros do modelo sobre a variável simulada. Para isso, a análise de sensibilidade possibilita identificar os parâmetros que mais influenciam no modelo e sua importância nos resultados gerados.

Por fim, a última etapa de verificação de um modelo hidrológico é a validação dos ajustes realizados nos parâmetros. A validação se resume em executar o modelo com os valores dos parâmetros determinados na calibração para um período de dados diferente ao utilizado na calibração (Arnold et al., 2012). Uma vez atendidos os critérios mínimos de qualidade, o modelo pode ser considerado adequado para a simulação do fluxo hidrossedimentológico em bacias experimentais.

Diante do potencial do SWAT como ferramenta de simulação e da carência de estudos aprofundados dessa ferramenta aplicada à pequenas bacias agrícolas no Brasil, buscou-se, com esse trabalho, aplicar a metodologia padrão de análise de sensibilidade, calibração e validação do SWAT visando avaliar sua eficácia para representação do fluxo hidrossedimentológico na bacia hidrográfica do rio Camboriú, Santa Catarina.

\section{MATERIAL E MÉTODOS}

\section{1. Área de estudo}

A bacia hidrográfica do rio Camboriú (BHRC) possui uma área de $195 \mathrm{~km}^{2}$ e abrange os municípios de Camboriú e Balneário Camboriú no estado de Santa Catarina (Figura 1). De acordo com a classificação de Köeppen, o clima dessa região é classificado como mesotérmico úmido subtropical, com temperatura média no mês mais frio inferior a $18^{\circ} \mathrm{C}$ e temperatura média no mês mais quente acima de $22^{\circ} \mathrm{C}$, com verões quentes e geadas pouco frequentes. A precipitação pluviométrica pode variar de $1.430 \mathrm{~mm}$ a $1.908 \mathrm{~mm}$ e existe a tendência de concentração das chuvas nos meses de verão, contudo sem estação seca definida.

Os solos predominantes da BHRC foram os Cambissolos, os Argissolos e os Gleissolos, ocupando, respectivamente, $48,7 \% ; 16,5 \%$ e $12,7 \%$ da área total da bacia. O mapeamento desses solos foi realizado com base em amostras coletadas em duas toposequências delimitadas ao longo da bacia. A partir da descrição dos perfis e dos resultados das análises físico-químicas, determinou-se os polígonos referentes às classes de solos com base na parametrização descrita em Embrapa (2013).

Entre as classes de uso e ocupação das terras, as áreas florestadas ocuparam $62,8 \%$ da área total da bacia, seguida pelas áreas com pastagem $(17,0 \%)$, áreas urbanas $(8,2 \%)$, arroz irrigado $(5,7 \%)$, reflorestamento $(2,5 \%)$, estradas rurais $(1,7 \%)$, solo exposto $(1,2 \%)$, massas d'água $(0,5 \%)$ e culturas anuais $(0,4 \%)$. O mapa de uso e ocupação foi gerado a partir de ortofotos correspondentes ao ano de 2012. A interpretação das imagens foi realizada através de ferramentas de processamento digital associada à coleta de dados utilizando GPS.

O modelo digital do terreno (MDT) foi obtido a partir do levantamento aerofotogramétrico de Santa Catarina realizado pela Secretaria de Desenvolvimento Sustentável (SDS/SC) no ano de 2010, adaptado para uma resolução espacial de 3 metros. O relevo da bacia hidrográfica foi dividido em cinco classes de declividade delimitadas com base no MDT e adaptadas à rotina de cálculos do modelo conforme classificação a seguir: 0 a 6\%; 6 a 20\%; 20 a 35\%; 35 a 48 e $>48 \%$.

Rev. Ambient. Água vol. 12 n. 2 Taubaté - Mar. / Apr. 2017 


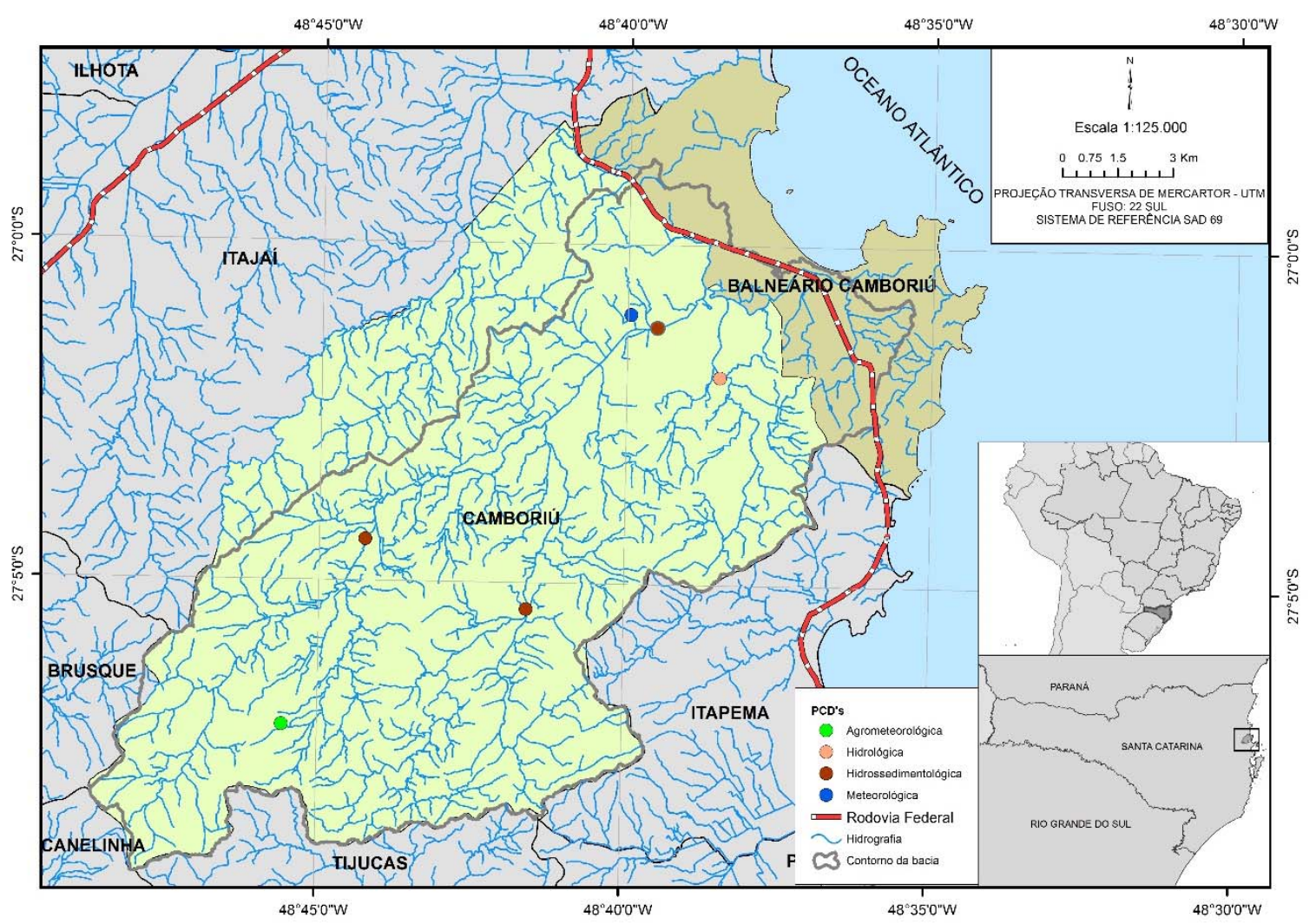

Figura 1. Mapa de localização da bacia hidrográfica do rio Camboriú.

\subsection{Modelo SWAT}

O SWAT é um modelo hidrológico fundamentado em características físicas de bacias hidrográficas, contínuo no tempo e com capacidade de simular longos períodos (Arnold et al., 2012). De acordo com os autores, o modelo foi desenvolvido com uma estrutura de comandos capaz de dividir a área em unidades homogêneas tendo como componentes aspectos climáticos, edáficos, topográficos, vegetativos e de manejo do solo, possibilitando a simulação de escoamento, sedimentos e nutrientes em bacias hidrográficas.

O componente hidrológico do modelo inclui sub-rotinas para determinação de escoamento superficial, percolação, fluxo lateral subsuperficial, fluxo de retorno do aquífero raso e evapotranspiração.

A perda de solo foi calculada a partir da Equação Universal de Perda de Solo Modificada - MUSLE (Williams, 1975). Essa metodologia utiliza a quantidade de escoamento para simular a erosão e a produção de sedimentos. A MUSLE é definida conforme Equação 1.

$$
\text { Sed }=11,8 \cdot\left(Q_{\text {surf }} \cdot q_{\text {peak }} \cdot \text { area }_{\text {hru }}\right)^{0,56} \cdot K_{U S L E} \cdot C_{U S L E} \cdot P_{U S L E} \cdot L S_{U S L E} \cdot C F R G
$$

em que:

Sed - perda do solo por unidade de área (ton);

Qsurf-é o volume de escoamento superficial $\left(\mathrm{mm} \mathrm{ha}^{-1}\right)$;

$q_{p e a k}$ - é o índice máximo de escoamento $\left(\mathrm{m}^{3} \mathrm{~s}^{-1}\right)$; area $a_{h r u}$-área de cada unidade de resposta hidrológica (ha);

$K_{U S L E}$ - fator de erodibilidade do solo;

CusLe - fator de uso e manejo;

PUSLE - fator práticas conservacionistas; 
$L S$ - fator topográfico; e

$C F R G$ - fator de fragmento bruto.

A vazão de pico foi calculada por uma modificação do Método Racional. O escoamento superficial foi obtido através do método Curva-Número do Soil Conservation Service (Neitsch et al., 2011).

O transporte de sedimentos através da rede de canais foi baseado nos processos de deposição e degradação. A deposição dentro do canal até o exutório da bacia foi determinada a partir da velocidade de deposição das partículas do sedimento (Melo Neto et al., 2014). A velocidade de sedimentação foi calculada a partir da lei de Stokes e a degradação do sedimento no canal foi fundamentada no conceito do poder de fluxo de Bagnold (1977).

A evapotranspiração potencial foi definida a partir do método de Penman-Monteith (Allen et al., 1989) e o ciclo hidrológico simulado pelo SWAT foi baseado na equação do balanço hídrico, conforme apresentado na Equação 2.

$$
S W_{t}=S W_{0}+\sum_{i=1}^{t}\left(R_{\text {day }}-Q_{\text {surf }}-E_{a}-W_{\text {seep }}-Q_{g w}\right)
$$

em que:

$$
\begin{aligned}
& S W_{t} \text { - conteúdo final de água no solo }(\mathrm{mm}) ; \\
& S W_{0} \text { - conteúdo de água no solo }(\mathrm{mm}) ; \\
& \mathrm{t} \text { - tempo (dias); } R_{\text {day }} \text { - precipitação }(\mathrm{mm}) ; \\
& Q_{\text {surf }} \text { - escoamento superficial }(\mathrm{mm}) ; \\
& E_{a} \text { - evapotranspiração }(\mathrm{mm}) ; \\
& W_{\text {seep }} \text { - percolação de água da camada simulada para a inferior }(\mathrm{mm}) ; \text { e } \\
& \text { Qgw }_{\mathrm{g}} \text { - escoamento lateral }(\mathrm{mm}) .
\end{aligned}
$$

\subsection{Dados hidrossedimentológicos}

Todos os dados hidrossedimentológicos foram coletados e transmitidos automaticamente a partir de plataformas de coleta de dados (PCD's) distribuídas ao longo da bacia hidrográfica (Figura 1).

No monitoramento meteorológico, a variável precipitação foi coletada nas seis PCD's (Figura 1). Já as variáveis temperatura e umidade relativa do ar foram registradas exclusivamente nas PCD's dos tipos meteorológica e agrometeorológica. Por fim, as demais variáveis (velocidade do vento e radiação solar) foram monitoradas somente na PCD meteorológica, conforme identificado na Figura 1. Todavia, a partir desses equipamentos registrou-se dados diários entre 2012 e 2015. A fim de ampliar a série de dados climatológicos, utilizou-se uma PCD instalada nas imediações da bacia hidrográfica. Assim, obteve-se um conjunto de dados compreendido entre os anos de 1981 e 2015.

$\mathrm{O}$ monitoramento hidrossedimentológico foi realizado a partir de sensores acoplados às PCD's do tipo hidrossedimentológicas (Figura 1). Nos três locais monitorados, determinou-se a vazão a partir do nível d'água associado às curvas-chave estabelecidas em campanhas de medição hidrológica. Ainda nesses pontos, foi determinado, indiretamente, o volume de sólidos transportados a partir do monitoramento contínuo de turbidez (NTU) e da correlação dessa variável com o conteúdo de sólidos suspensos total (SST) medido ao longo do período de 2012 a 2015. Por fim, a quantidade de sólidos transportados foi determinada pela multiplicação do conteúdo de SST pela vazão acumulada diariamente. 


\subsection{Calibração e validação do modelo}

A calibração do modelo SWAT foi realizada de maneira automatizada pelo software SWAT-CUP. Dentre as opções disponíveis no SWAT-CUP para os procedimentos de calibração e análise de incertezas, optou-se pelo método SUFI2 (Sequential Uncertainty Fitting). Nesse processo é utilizado o método de hipercubo latino para a definição dos parâmetros de análise, e o usuário define o intervalo para a calibração e o número de simulações a serem realizadas, a fim de definir o melhor ajuste (Abbaspour et al., 2007).

Para a etapa de calibração, foi selecionado o intervalo de dados diários de vazão e transporte de sedimentos compreendidos entre 01/01/2014 e 31/12/2014, enquanto que para a validação do ajuste do modelo foi selecionado o período de 01/01/2015 a 31/12/2015.

\subsection{Funções objetivo}

Para avaliar o desempenho do SWAT foram utilizadas as seguintes estatísticas de precisão: coeficiente de Nash-Sutcliffe (NSE), Equação 3, e o coeficiente de massa residual (CMR), descrito na Equação 4.

A correlação perfeita entre dados simulados e observados é expressa por um valor de $\mathrm{NSE}=1$ (Equação 3). Valores de NSE $<0$ demonstram que a utilização da média dos valores observados é melhor do que os resultados simulados (Brighenti et al., 2016).

$$
N S E=\left[\frac{\sum_{i=1}^{n}\left(Q_{i}^{o b s}-Q_{i}^{s i m}\right)^{2}}{\sum_{i=1}^{n}\left(Q_{i}^{\text {obs }}-Q^{\text {mean }}\right)^{2}}\right]
$$

em que:

NSE - coeficiente de Nash e Sutcliffe;

$n$ - número total de observações;

$Q_{i}{ }^{\text {obs }}$ - vazão observada;

$Q_{i}^{\text {sim }}$ - vazão simulada; e

$\mathrm{Q}^{\text {mean }}$ - média da vazão observada no período.

A tendência média entre valores simulados e medidos é expressa pelo CMR (Equação 4). $\mathrm{CRM}=0$ indica uma relação perfeita entre os dados, valores positivos indicam uma tendência de subestimativa do modelo, enquanto que valores negativos sinalizam para a superestimativa da variável simulada.

$$
C M R=\left[\frac{\sum_{i=1}^{n} Q_{i}^{s i m}-\sum_{i=1}^{n} Q_{i}^{o b s}}{\sum_{i=1}^{n} Q_{i}^{s i m}}\right]
$$

em que:

$C M R$ é o coeficiente de massa residual;

$Q_{i}{ }^{\text {sim }}$ é a vazão simulada pelo modelo; e

$Q_{i}{ }^{o b s}$ é a vazão medida.

Para classificação do desempenho do SWAT foi adotada a escala definida por Santhi et al. (2001): Muito bom: $N S E \geq 0,65$; Bom: 0,65 $>N S E \geq 0,54$; satisfatório: $0,54>N S E \geq 0,50$. Para o $C M R$ foi utilizada escala proposta por Liew et al. (2007): Muito bom: $|C M R| \leq 10 \%$; Bom: $10 \%<|C M R| \leq 15 \%$; Satisfatório: $15 \%<|C M R| \leq 25 \%$; Inadequado: $|C M R|>25 \%$. 


\section{RESULTADOS E DISCUSSÃO}

Na Tabela 1 estão listados os parâmetros classificados como mais sensíveis sobre as variáveis estudadas na escala temporal diária. Esses parâmetros foram identificados através da análise de sensibilidade realizada com o software SWAT-CUP. A lista completa dos parâmetros testados foi elaborada a partir de uma revisão bibliográfica em trabalhos que utilizaram os mesmos procedimentos.

Os parâmetros mostrados na Tabela 1 representam variáveis de difícil medição, ademais, existem poucas informações disponíveis acerca dessas variáveis para bacias hidrográficas brasileiras, principalmente para as de pequeno porte. Assim, o intervalo de valores adotado no processo de calibração foi definido com base em trabalhos dessa natureza (Santhi et al., 2001; Andrade et al., 2012; Arnold et al., 2012; Brighenti et al., 2016).

Tabela 1. Parâmetros utilizados na calibração do modelo SWAT com os valores ajustados e as respectivas faixas de variação.

\begin{tabular}{|c|c|c|c|c|}
\hline Ranking & Parâmetro & Descrição & $\begin{array}{l}\text { Valor } \\
\text { ajustado }\end{array}$ & $\begin{array}{l}\text { Faixa de } \\
\text { variação }\end{array}$ \\
\hline 1 & Lat_Time & Tempo necessário para o retorno do fluxo lateral (dias) & 42 & 0 a 180 \\
\hline 2 & Esco & $\begin{array}{l}\text { Fator de compensação da taxa de evaporação de água no } \\
\text { solo }\end{array}$ & 0,90 & 0 a 1 \\
\hline 3 & Gwqmin & $\begin{array}{l}\text { Nível limite da água no aquífero raso para a ocorrência } \\
\text { de fluxo de base }(\mathrm{mm})\end{array}$ & 1.000 & 0 a 5.000 \\
\hline 4 & Revapmn & $\begin{array}{l}\text { Profundidade limite de água no solo para a ocorrência } \\
\text { de ascensão da água à zona não saturada }(\mathrm{mm})\end{array}$ & 1.000 & 0 a 1.000 \\
\hline 5 & Alpha_BF & Constante de recessão do escoamento de base (dias) & 0,60 & 0 a 1 \\
\hline 6 & Gw_delay & $\begin{array}{l}\text { Intervalo de tempo necessário para a recarga do aquífero } \\
\text { (dias) }\end{array}$ & 0,65 & 0 a 500 \\
\hline
\end{tabular}

Uma etapa importante do processo de calibração e validação de um modelo hidrológico é a análise dos hidrogramas gerados e a comparação entre os resultados medidos e simulados. $\mathrm{Na}$ Figura 2, são apresentados os hidrogramas gerados nas fases de calibração e validação do SWAT. O período correspondente ao ano de 2014 foi selecionado para compor a etapa de calibração (Figura 2A), enquanto que o ano de 2015 foi utilizado para a validação do SWAT (Figura 2B). Em ambos os períodos, pode-se identificar uma aderência satisfatória entre os hidrogramas medidos e simulados.

As funções objetivo utilizadas para atestar a eficácia do SWAT corroboraram a sua eficiência para representar o fluxo hidrológico na BHRC. Na etapa de calibração, o NSE foi de 0,66 (Figura 2A). De acordo com a escala adotadas por Santhi et al. (2001) e com base nos valores de NSE, o modelo pode ser classificado como muito bom para a representação do fluxo de vazão na BHRC em escala de tempo diária. Na etapa de validação, o NSE foi de 0,89 (Figura 2B), ou seja, assim como na etapa de calibração, o modelo foi considerado muito bom para representação dessa variável (Santhi et al., 2001).

O CMR é a função objetivo que representa o percentual de viés entre dados simulados e medidos. Os resultados obtidos indicam um pequeno desvio em ambas as etapas. Na calibração, houve uma subestimativa de $3 \%$, e na fase de validação SWAT a subestimativa foi de $14 \%$ (Figura 2). De acordo com Liew et al. (2007) esses resultados indicam uma adequação muito boa do modelo SWAT para representação do fluxo hidrológico na primeira etapa e um desempenho bom do modelo na etapa de validação.

Com base nas funções objetivo utilizadas, pode-se concluir que o SWAT apresentou boa precisão nas estimativas de vazão representadas pelos valores de NSE. Além disso, foi possível constatar que as estimativas não apresentaram tendência significativa, dadas pelos baixos 
valores de CMR. Dessa forma, foi possível comprovar que os parâmetros foram devidamente ajustados à $\mathrm{BHRC}$ e não produziram estimativas com viés.

A quantidade de sólidos transportados diariamente ao longo da seção de controle é apresentada na Figura 3. Os sedimentogramas gerados na etapa de calibração (Figura 3A) e validação (Figura $3 \mathrm{~B}$ ) destacam o elevado grau de aderência entre as variáveis medidas e simuladas nos dois períodos. Na calibração, o NSE foi de 0,80 e o $C M R$ foi de 0,24 , ou seja, de acordo com Santhi et al. (2001), o SWAT foi classificado como muito bom para simulação da quantidade de sólidos transportada, entretanto, o CMR de 0,24 indica uma acentuada subestimativa da variável simulada. De acordo com a escala adotada por Liew et al. (2007), o modelo é classificado como satisfatório e com um viés de subestimativa da variável estudada, porém, dentro dos limites relatados na literatura. Vale destacar que devido a limitação de dados disponíveis de sólidos transportados, utilizou-se um período de nove meses na etapa de calibração. Além disso, observou-se que no período de calibração as vazões foram relativamente baixas, caracterizando um período de recessão, o que pode ter prejudicado a representação desta variável pelo SWAT.

Todavia, na etapa de validação, o desempenho do SWAT para simular a quantidade de sólidos transportada foi melhor do que na calibração, com NSE de 0,88 e CMR de 0,12. Tais resultados possibilitam classificar o SWAT como muito bom e com uma tendência de subestimativa de $12 \%$ da variável simulada, entretanto, com um viés não significativo.
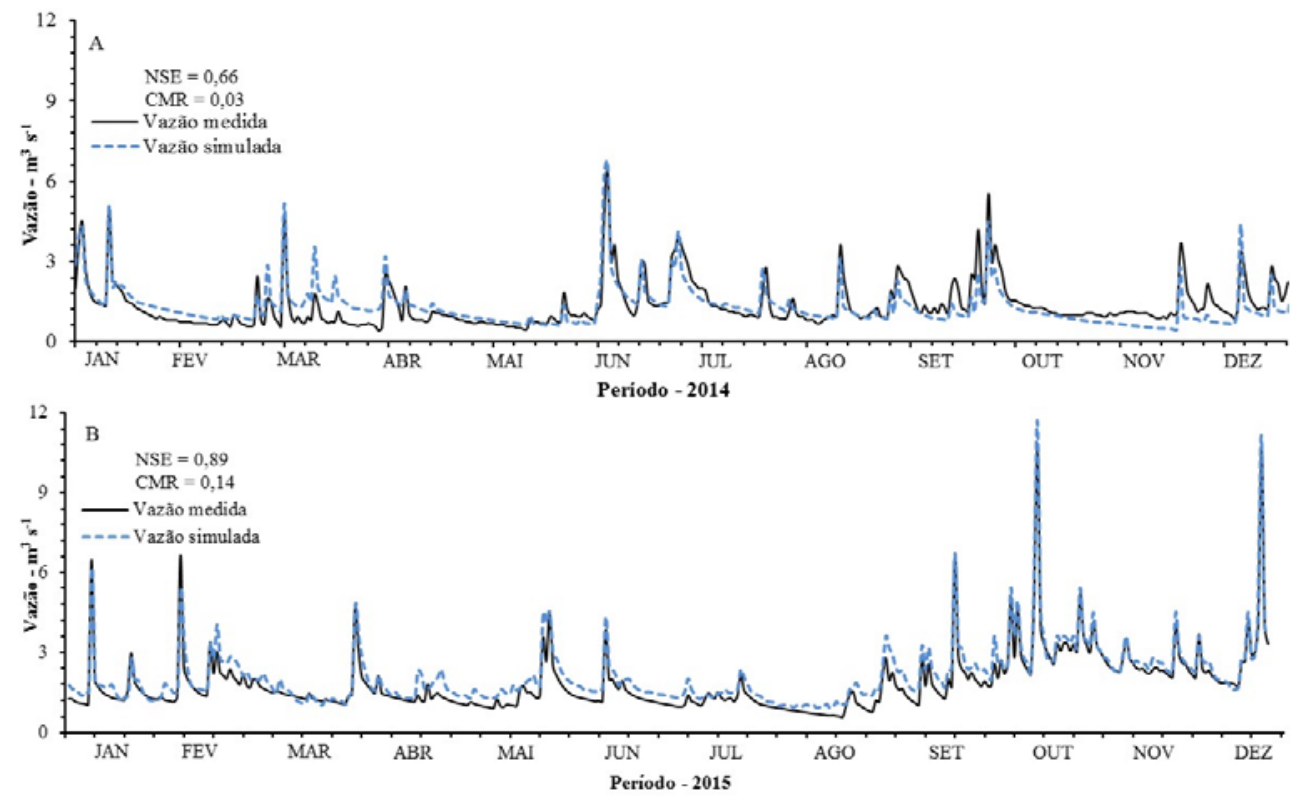

Figura 2. Hidrograma medido e simulado para a BHRC no período de calibração (A) e validação (B), na seção de controle.

Um aspecto importante associado à observação dos hidrogramas e sedimentogramas (Figuras 2 e 3) refere-se à dificuldade de simulação das vazões de cheia e, consequentemente, dos picos de sólidos transportados no período. Outros autores relataram as mesmas dificuldades e consideraram como aspectos limitantes a simplificação da distribuição das chuvas, o intervalo de simulação adotado na modelagem maior que o tempo de concentração das sub-bacias e o monitoramento hidrológico e climático com intervalo de coleta diário. Tais fatores, via de regra, limitam a resposta dos modelos às vazões máximas, dado ao reduzido tempo de concentração das sub-bacias (Bormann et al., 2007; Viola et al., 2009). 

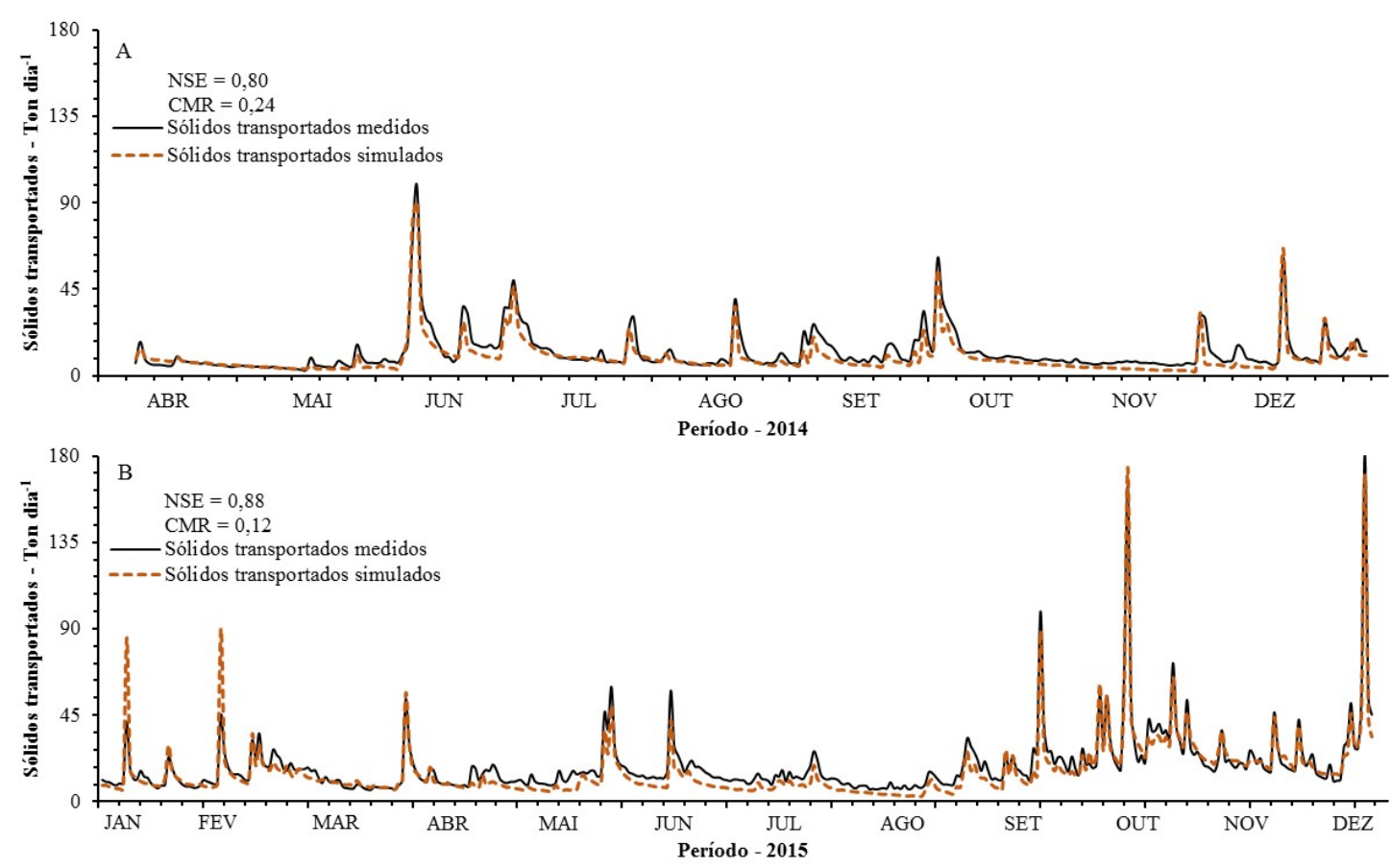

Figura 3. Sedimentograma medido e simulado para a BHRC no período de calibração (A) e validação (B) na seção de controle.

Entretanto, apesar da complexidade de simulações em bacia hidrográficas de pequeno porte e de algumas variações nas simulações dos picos de vazão e de sólidos transportados, verificou-se uma boa aderência dos hidrogramas e sedimentogramas simulados com relação aos medidos. Além disso, nos períodos de recessão, observou-se grande proximidade entre dados medidos e simulados, reforçando os resultados obtidos pelas funções objetivo.

Outra análise que pode ser feita com relação à calibração e validação de modelos hidrológicos diz respeito à capacidade de gerar séries de dados confiáveis para a elaboração das curvas de permanência. Essa funcionalidade dos modelos pode ser fundamental para a elaboração dos planos de bacias hidrográficas. Assim, os modelos hidrológicos podem ser úteis para uma gestão eficiente de recursos hídricos, principalmente em bacias hidrográficas de pequeno porte ou em regiões onde se tenham conflitos pelo uso da água.

De acordo com Viola et al. (2009), a partir de séries temporais de dados simulados pelos modelos hidrológicos, pode-se proceder a análise de vazões de referência como vazões de pico, de recessão e de referência para outorga d'água.

Para estimativa de vazões de referência para outorga há uma série de limitações para pequenas bacias, uma vez que é praticamente inexistente a disponibilidade de dados hidrológicos de vazão nesta escala no Brasil (Andrade et al., 2012). Nesses casos, as curvas de permanência geradas a partir de modelos hidrológicos constituem ferramentas importantes para a determinação da disponibilidade hídrica em bacias hidrográficas.

Na Figura 4 estão ilustradas as curvas de permanência observada e simulada pelo modelo para as variáveis vazão (Figura 4A) e sólidos transportados (Figura 4B). Cabe ressaltar que as curvas de permanência foram geradas com a série de dados utilizada na etapa de calibração e validação, ou seja, compreendem o período de 2014 a 2015.

A análise visual das curvas de permanência de vazão (Figura 4A) reforça o fato de ter ocorrido uma subestimativa de variável simulada. Todavia, o percentual de subestimativa esteve dentro de limites aceitáveis, principalmente para vazões de referência como a Q95\%, Q90\%, Q50\%, Q10\% e Q5\%, todas essas utilizadas como referência em projetos de hidráulica e em estudos relacionados aos recursos hídricos. Para as vazões de referência listadas 
anteriormente, os percentuais de subestimativa foram respectivamente de $16,9 \%, 14,8 \%$, $15,2 \%, 12,5 \%$ e $9,5 \%$.

Os resultados apresentaram o mesmo comportamento para a curva de permanência de sólidos transportados (Figura 4B), entretanto com magnitudes diferentes. Para valores com probabilidade de ocorrência de $95 \%$, o modelo subestimou a variável em $31,8 \%$, enquanto que para probabilidades de ocorrência menores como $10 \%$ ou $5 \%$, o modelo subestimou a variável simulada em $8,9 \%$ e $11,2 \%$ respectivamente, comparado ao valor medido.

De maneira geral, esses resultados indicaram que o SWAT apresentou uma tendência de subestimativa das variáveis simuladas, entretanto, dentro de limites aceitáveis dada a complexidade dos fenômenos envolvidos nos processos naturais que determinam o fluxo de água e sedimentos em bacias hidrográficas. Assim, ficou evidente o potencial do SWAT para simular de forma adequada as curvas de permanência na BHRC e demonstrando sua qualidade como ferramenta auxiliar na gestão dos recursos hídricos.

Com base em todas as análises e comparações realizadas entre os dados medidos e simulados pelo SWAT, foi possível constatar que o modelo apresentou alta precisão e adequação para simulação de vazões e de sólidos transportados em escala de tempo diária na BHRC. Ademais, foi possível verificar que os resultados dessa pesquisa estão de acordo com inúmeros trabalhos realizados no Brasil e no exterior, no tocante à utilização de modelos matemáticos para simulação do regime hidrossedimentológico em bacias hidrográficas experimentais.
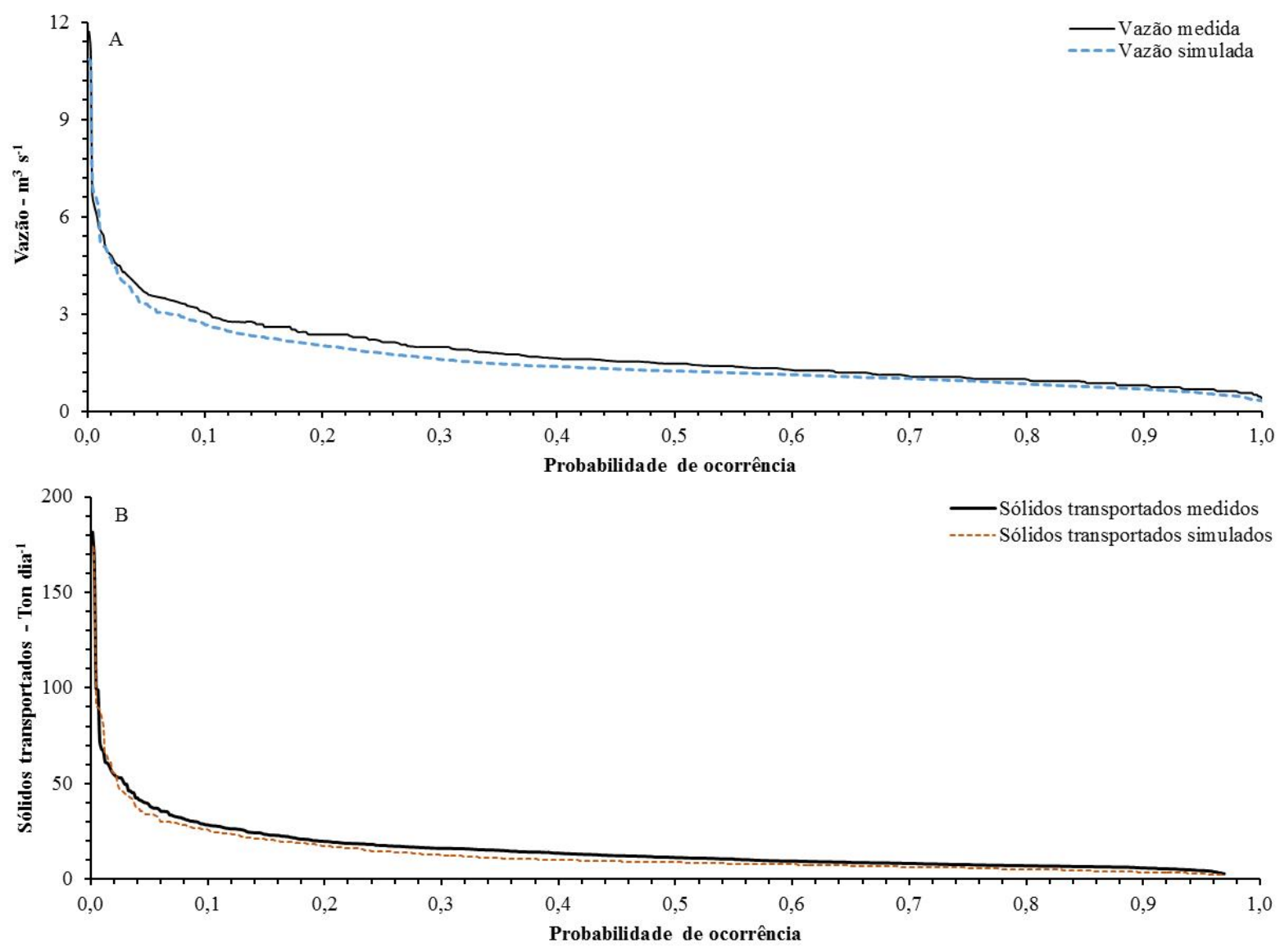

Figura 4. Curvas de permanência observadas e medidas para as variáveis vazão (A) e sólidos transportados (B) através seção controle instalada na BHRC. 


\section{CONCLUSÃO}

O modelo SWAT mostrou-se eficiente para as simulações hidrossedimentológicas na BHRC para a escala de tempo diária. Contudo, foi preciso realizar a sua calibração para que as simulações atendessem os critérios de qualidade estabelecidos.

Os resultados obtidos após a calibração do SWAT demonstraram uma tendência de subestimativa das variáveis simuladas, entretanto, dentro de limites aceitáveis, principalmente, devido à complexidade dos fenômenos envolvidos nos processos naturais que determinam o fluxo de água e sedimentos em bacias hidrográficas de pequeno porte.

Além dos resultados satisfatórios demonstrados pelas funções objetivo, foi possível atestar a eficiência do modelo para simulação das curvas de permanência das variáveis estudadas, caracterizando o SWAT como uma ferramenta importante para o processo de gestão de bacias hidrográficas como a do rio Camboriú.

\section{REFERÊNCIAS}

ABBASPOUR, K. C.; YANG, J.; MAXIMOV, I.; SIBER, R.; BOGNER, K.; MIELEITNER, J. et al. Modelling of hydrology and water quality in the pre-alpine/alpine Thur watershed using SWAT. Journal of Hydrology, v. 333, n. 2-4, p. 413-430, 2007. http://dx.doi.org/10.1016/j.jhydrol.2006.09.014

ALLEN, R. G.; JENSEN, J. L.; BURMAN, R. D. Operational Estimates of Reference Evapotranspiration. Agronomy Journal, v. 81, n. 4, p. 650-662, 1989. http://dx.doi.org/10.2134/agronj1989.00021962008100040019x

ANDRADE, M. A.; de MELO, C. R.; BESKOW, S. Simulação hidrológica em uma bacia hidrográfica representativa dos Latossolos na região Alto Rio Grande, MG. Revista Brasileira de Engenharia Agrícola e Ambiental, v. 17, n. 1, p. 69-76, 2012. http://dx.doi.org/10.1590/S1415-43662013000100010

ARNOLD, J. G.; MORIASI, D. N.; GASSMAN, P. W.; ABBASPOUR, K. C.; WHITE, M. J.; SRINIVASAN, R. et al. SWAT: Model use calibration and validation. Transactions of ASABE, v. 55, n. 4, p. 1494-1508, 2012.

BAGNOLD, R. A. Bed load transport by natural rivers. Water Resources Research, v. 13, n. 1, p. 303-312, 1977. http://dx.doi.org/10.1029/WR013i002p00303

BORMANN, H.; BREUER, L.; GRÄFF, T.; HUISMAN, J. A. Analyzing the effects of soil properties changes associated with land use changes on the simulated water balance: A comparison of three hydrological catchment models for scenario analysis. Ecological Modelling, v. 209, n. 1, p. 29-40, 2007.

http://dx.doi.org/10.1016/j.ecolmodel.2007.07.004

BRESSIANI, D. A.; GASSMAN, P. W.; FERNANDES, J. G.; GARBOSSA, L.; SRINIVASAN, R.; BONUMA, N. B. et al. A review of Soil and Water Assessment Tool (SWAT) applications in Brazil: challenges and prospects. International Journal of Agricultural and Biological Engineering, v. 8, n. 3, p. 1-27, 2015.

BRIGHENTI, T. M.; BONUMÁ, N. B.; CHAFFE, P. L. B. Calibração hierárquica do modelo Swat em uma bacia hidrográfica Catarinense. Revista Brasileira de Recursos Hídricos, v. 21, n. 1, p. 53-64, 2016. http://dx.doi.org/10.21168/rbrh.v21n1.p53-64 
EMPRESA BRASILEIRA DE PESQUISA AGROPECUÁRIA - EMBRAPA. Centro Nacional de Pesquisa de Solos. Sistema brasileiro de classificação de solos. 3 . ed. Brasília, 2013. 353 p.

LIEW, M. W.; VEITH, T. L.; BOSCH, D. D.; ARNOLD, J. G. Suitability of SWAT for the Conservation effects assessment project: A comparison on USDA-ARS watersheds. Journal of Hydrology Resources, v. 12, n. 2, p. 173-189, 2007. http://dx.doi.org/10.1061/(ASCE)1084-0699(2007)12:2(173)

MElO NETO, J. de O.; SILVA, A. M. da; MELlO, C. R. de; MELlO JÚNIOR, A. V. Simulação hidrológica escalar com o modelo SWAT. Revista Brasileira de Recursos Hídricos, v. 19, n. 1, p. 177-188, 2014. http://dx.doi.org/10.21168/rbrh.v19n1.p177-188

MULETA, M. K.; NICKLOW, J. W. Sensitivity and uncertainty analysis coupled with automatic calibration for a distributed watershed model. Journal of Hydrology, v. 306, p. 127-145, 2005. http://dx.doi.org/10.1016/j.jhydrol.2004.09.005

NEITSCH, S. L.; ARNOLD, J. G.; KINIRY, J. R.; WILLIAMS, J. R. Soil and Water Assessment Tool: Theoretical documentation - version 2009. Texas: Grassland, Soil and Water Research Laboratory - Agricultural Research Service; Blackland Research Center - Texas AgriLife Research, 2011.618 p.

SANTHI, C.; ARNOLD, J. G.; WILLIAMS, J. R.; DUGAS, W. A.; SRINIVASAN, R.; HAUCK, L. M. Validation of the SWAT model on a large river basin with point and nonpoint sources. Journal of American Water Resources Association, v. 37, n. 5, p. 1169-1188, 2001. http://dx.doi.org/10.1111/j.1752-1688.2001.tb03630.x

VEITH, T. L.; LIEW, M. W. VAN; BOSCH, D. D.; ARNOLD, J. G. Parameter sensitivity and uncertainty in SWAT: A comparison across five USDA-ARS watersheds. Transactions of ASABE, v. 53, p. 1477-1486, 2010.

VIOLA, M. R.; de MELlO, C. R.; ACERBI Jr., F. W.; DA SILVA, A. M. Modelagem hidrológica na bacia hidrográfica do Rio Aiuruoca, MG. Revista Brasileira de Engenharia Agrícola e Ambiental, v. 13, n. 5, p. 581-590, 2009. http://dx.doi.org/10.1590/S1415-43662009000500011

WILLIAMS, J. R. Sediment routing for agricultural watersheds. Water Resourses Bulletin, v. 11 n. 5, p. 965-974, 1975. http://dx.doi.org/10.1111/j.1752-1688.1975.tb01817.x 


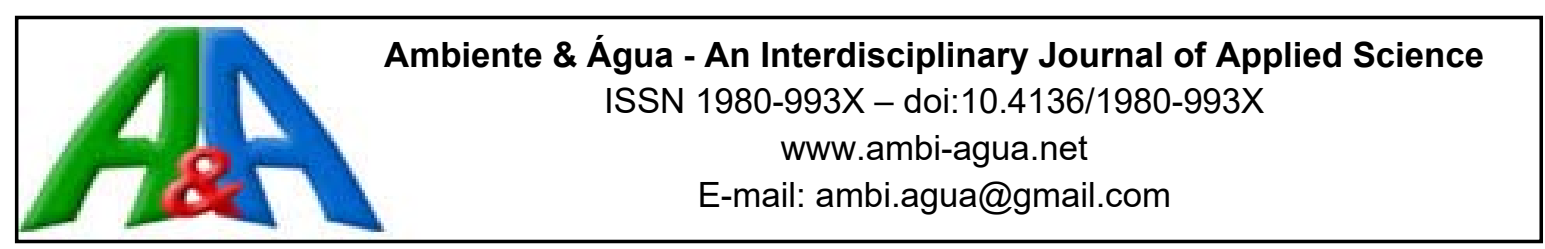

\title{
Caracterização estacional das variáveis físicas, químicas, biológicas e ecotoxicológicas em um trecho do Rio Paraíba do Sul, SP, Brasil
}

\author{
doi:10.4136/ambi-agua.1949 \\ Received: 30 May 2016; Accepted: 23 Jan. 2017 \\ Lucas Gonçalves Queiroz*; Flávio Teixeira da Silva; \\ Teresa Cristina Brazil de Paiva
Universidade de São Paulo (USP), Escola de Engenharia de Lorena (EEL), Lorena, SP, Brasil
Departamento de Bioquímica
*Autor correspondente: e-mail: lucasgoncalvesqueiroz@gmail.com, flavio@debiq.eel.usp.br, teresa@debiq.eel.usp.br

\section{RESUMO}

O Rio Paraíba do Sul está localizado em uma região brasileira com elevada densidade populacional e grande importância socioeconômica. Em seu trecho paulista, o rio encontra-se impactado e com a qualidade da água comprometida devido à retirada da vegetação ciliar, o aumento de áreas impermeáveis, a atividade industrial, a utilização de insumos agrícolas e, principalmente, pelo lançamento de esgotos sem tratamento adequado. O objetivo deste trabalho foi caracterizar, durante as estações seca e chuvosa, a qualidade da água do Rio Paraíba do Sul quanto as variáveis físicas, químicas, biológicas e ecotoxicológicas em seu trecho médio superior. As coletas das amostras foram realizadas trimestralmente entre os meses de agosto de 2013 e agosto de 2014. Foram determinadas variáveis: $\mathrm{pH}$, temperatura, condutividade, turbidez, oxigênio dissolvido (OD), demanda bioquímica de oxigênio (DBO), demanda química de oxigênio (DQO), fósforo total (PT), sólidos totais (ST), coliformes termotolerantes (CT), clorofila-a, efeito tóxico agudo (ETA) e crônico (ETC) e Índice de Estado Trófico (IET). Os resultados referentes à qualidade das águas mostraram que as variáveis $\mathrm{OD}, \mathrm{PT}$ e CT estavam em desacordo com o estabelecido para um rio classe 2, conforme a resolução CONAMA 357/2005 (2005). Algumas variáveis apresentaram uma redução ao longo do período avaliado, como DBO $(42,4 \%)$, ST $(31,1 \%)$ e DQO $(21,3 \%)$. No período chuvoso foram observados ETA e ETC. A análise estatística mostrou que a precipitação pluviométrica possui grande influência sobre OD, DBO, ST, clorofila- $a$, ETA e ETC, atuando como um possível agente intensificador de impactos sobre este manancial.

Palavras-chave: contaminação da água, ecotoxicologia aquática, organismos-teste, precipitação pluviométrica, uso do solo.

\section{Seasonal characterization of the physical, chemical, biological and ecotoxicological variables in a stretch of the Paraíba do Sul River, SP, Brazil}

\section{ABSTRACT}

The Paraíba do Sul River is located in a Brazilian region with high population density and great socio-economic importance. In the São Paulo stretch, the river is impacted and water 
quality is impaired due to the removal of riparian vegetation, increases in impermeable surfaces, industrial activity, the use of agricultural inputs, and the release of wastewater without proper treatment. This study characterized the physical, chemical, biological and ecotoxicological variables associated with the water quality of the Paraíba do Sul River in the upper-middle stretch during the dry and wet seasons. The sampling was carried out quarterly between August 2013 and August 2014. The variables evaluated were $\mathrm{pH}$, temperature, conductivity, turbidity, dissolved oxygen (OD), biochemical oxygen demand (DBO), chemical oxygen demand (DQO), total phosphorus (PT), total solids (ST), fecal coliforms (CT), chlorophyll-a, acute toxic effect (ETA) and chronic toxic effect (ETC) and Trophic State Index (IET). The results showed that the variables OD, PT and CT were not in accordance with those established for a Class 2 aquatic environment, according to the National Counsel for the Environment 357/2005 (Portuguese acronym "CONAMA"). Some variables showed a reduction during the evaluated period, such as DBO (42.4\%), ST (31.1\%) and DQO (21.3\%). Both ETA and ETC were observed in the rainy period. The statistical analysis showed that the rainfall has an influence on OD, DBO, ST, chlorophyll- $a$, ETA and ETC, acting as a possible intensifier agent of impacts on the river.

Keywords: aquatic ecotoxicology, land use, pluviometric precipitation, test organisms, water contamination.

\section{INTRODUÇÃO}

A Bacia do Rio Paraíba do Sul, localizada no sudeste brasileiro entre os estados de São Paulo, Minas Gerais e Rio de Janeiro, constitui um importante eixo de desenvolvimento socioeconômico no país, devido à elevada densidade populacional, grau de urbanização e industrialização (Demanboro, 2015).

O grande desenvolvimento da região, entretanto, é motivo de problemas em relação à disponibilidade de água. Apesar de apresentar uma das maiores demandas hídricas do país, a região possui uma das menores disponibilidades relativas (ANA, 2012).

Áreas urbanizadas e agrícolas possuem grande potencial de degradação de corpos hídricos, sobretudo quando há um baixo grau de saneamento e mitigação de agentes causadores de impacto ambiental. Atividades nestas áreas alteram a qualidade da água, pela adição de nutrientes, matéria orgânica e contaminantes (Carvalho et al., 2015, Passig et al., 2015).

Ao longo dos anos, o Rio Paraíba do Sul tem sido constantemente impactado devido ao intenso uso e ocupação do solo, resultando na retirada da vegetação e aumento de áreas impermeáveis, utilização de insumos agrícolas e, principalmente, pelo lançamento de efluentes domésticos e industriais sem tratamento adequado. Estes impactos promovem a redução da qualidade da água deste manancial afetando diretamente a disponibilidade hídrica, as comunidades aquáticas e o abastecimento público (Malafaia et al., 2012; CEIVAP, 2014).

O objetivo deste estudo foi avaliar os efeitos da precipitação e correlacioná-los às variáveis associadas à qualidade da água do Rio Paraíba do Sul, em seu trecho paulista, frente aos impactos causados pelas atividades humanas. Esta região possui grande importância socioeconômica devido à elevada densidade populacional, presença de indústrias e áreas destinadas à agricultura. Estas condições resultam na perda da qualidade da água, devido a elevada demanda hídrica, lançamento de poluentes e retirada da cobertura vegetal. Deste modo, um maior conhecimento acerca deste corpo hídrico pode auxiliar na promoção de formas de gestão para preservação e consequente melhoria da qualidade da água. 


\section{METODOLOGIA}

\section{1. Área de estudo}

O Rio Paraíba do Sul (RPS) nasce pela confluência dos rios Paraibuna e Paraitinga, no Estado de São Paulo, percorre cerca de $1.150 \mathrm{Km}$ e deságua no município de São João da Barra, no Estado do Rio de Janeiro (CEIVAP, 2014, Marengo e Alves, 2005). Esta região apresenta elevada densidade populacional, presença de indústrias e áreas cultiváveis.

O trecho avaliado está localizado entre os municípios de Aparecida e Cachoeira Paulista, onde suas águas são destinadas principalmente ao abastecimento, diluição de esgotos, irrigação e geração de energia, sendo classificado como Classe 2. A Figura 1 apresenta os diferentes usos do solo do trecho, os quais refletem diretamente na qualidade da água.

A partir das imagens de satélite Landsat TM-5 (Figura 1), foi possível calcular a frequência relativa das classes de cobertura do solo da região. Em seu trecho paulista, as áreas urbanizadas correspondem a 9,3\% da bacia e as áreas agrícolas e de pastagens correspondem juntas a 36,9\%. Estas atividades estão localizadas principalmente próximas às margens do RPS, causando impactos sobre o corpo hídrico e em suas áreas adjacentes.

\subsection{Coleta de amostras}

Foram realizadas cinco coletas de água em oito pontos próximos à montante $(\mathrm{M}) \mathrm{e}$ à jusante (J) do perímetro urbano de quatro municípios paulistas: Aparecida (APDM (-22.858095, 45.254591) e APDj (-22.842567, -45.234106)), Guaratinguetá (GUAm (-22.811946, 45.194412) e GUAJ (-22.790639, -45.178652)), Lorena (LORM (-22.742899, -45.144604) e LORJ (-22.698778, -45.115863)) e Cachoeira Paulista (CPTM (-22.634923, -44.991708) e CPTJ (-22.664032, -45.023458)). A localização dos pontos permite observar a contribuição de cada município avaliado sobre a poluição do RPS. As coletas foram realizadas trimestralmente entre os meses de agosto de 2013 e agosto de 2014 e preservadas conforme critérios determinados pela norma ABNT NBR 9.898/87 (ABNT, 1987). Os dados pluviométricos foram fornecidos pelo Departamento de Águas e Energia Elétrica (DAEE) dos municípios de Lorena e Guaratinguetá e utilizados para caracterizar os períodos de precipitação na região (Figura 2).

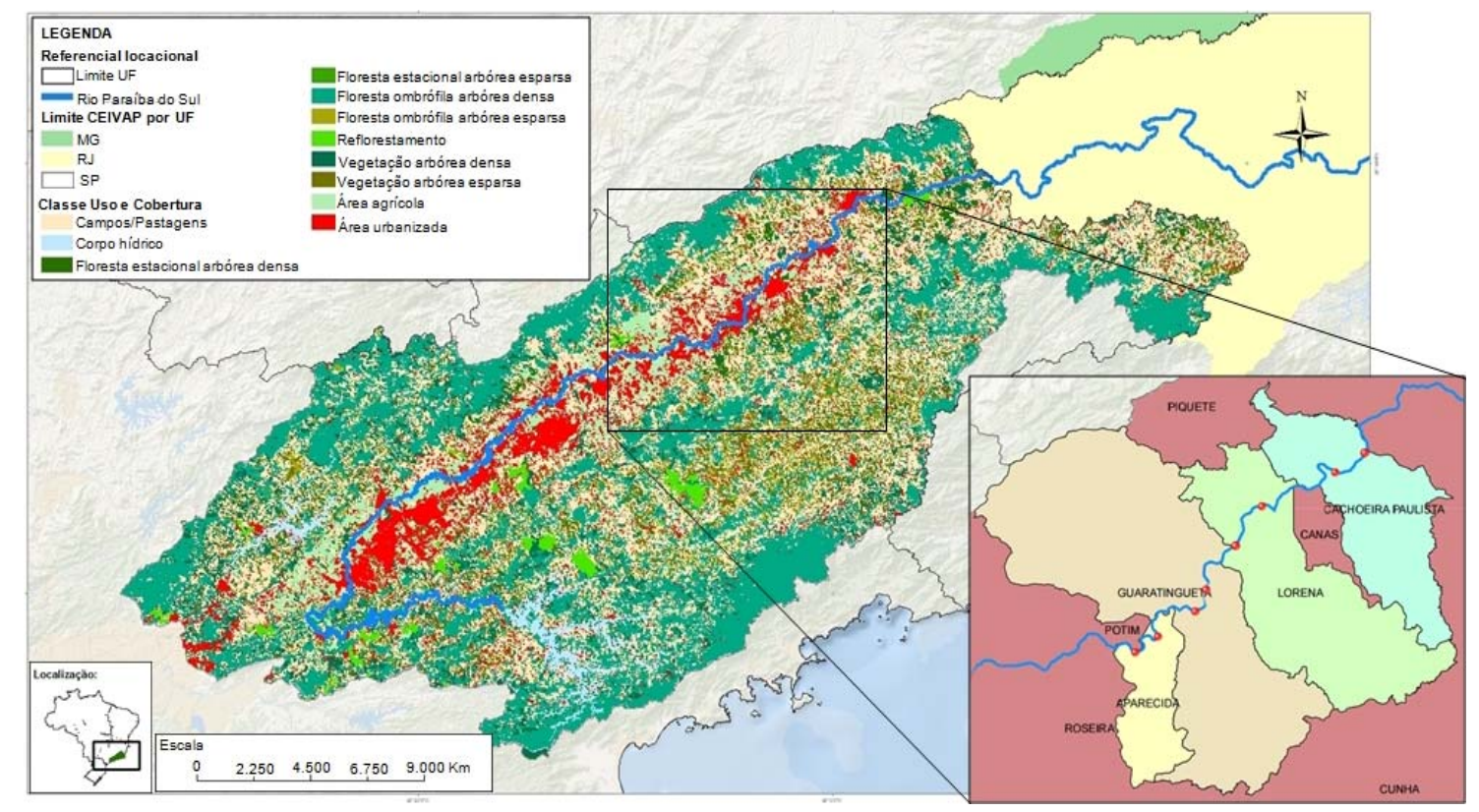

Figura 1. Uso e ocupação do solo no trecho paulista da Bacia do Rio Paraíba do Sul (com base na classificação de imagens Landsat TM 5). 


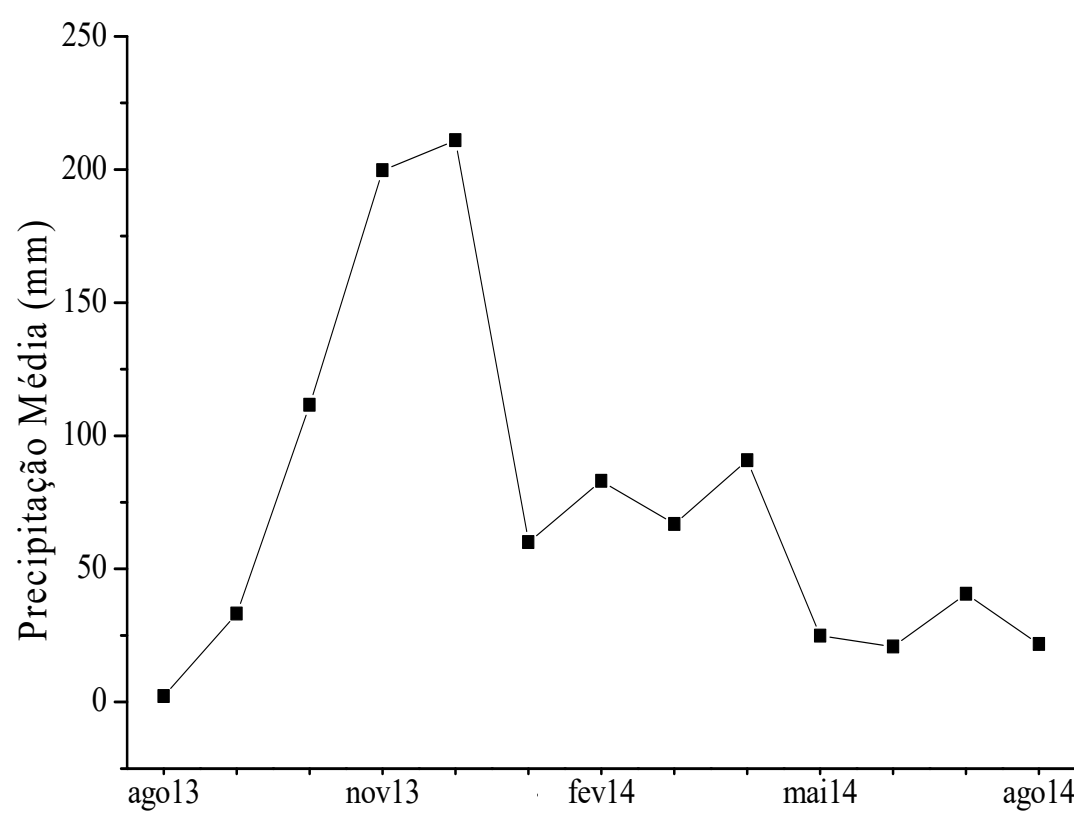

Figura 2. Média mensal dos valores de precipitação $(\mathrm{mm})$ observados entre agosto de 2013 e agosto de 2014.

Fonte: DAEE (2014)

\subsection{Análises físicas, químicas, biológicas e ecotoxicológicas}

No momento da coleta, foram determinadas as variáveis $\mathrm{pH}$, a condutividade e a temperatura com auxílio de uma sonda multiparamétrica (HANNA modelo HI9811-5). Os valores de oxigênio dissolvido (OD), demanda bioquímica de oxigênio (DBO), demanda química de oxigênio (DQO) e a concentração de fósforo total (PT) foram determinadas de acordo com APHA ET AL. (2015). A turbidez foi determinada a partir de turbidímetro (TB 1000 ), os sólidos totais (ST) pelo método gravimétrico e o nitrogênio total (NT) pelo método Kjeldahl adaptado (Galvani e Gaertner, 2006).

A quantificação de coliformes termotolerantes (CT) foi realizada segundo APHA ET AL. (2015), e para a concentração de clorofila- $a$ utilizou-se o método proposto por Lorezen (1967).

Bioensaios para determinação de efeito tóxico agudo (ETA) com Daphnia similis e efeito tóxico crônico (ETC) com Raphidocelis subcapitata foram realizados conforme as normas ABNT NBR 12.713/16 e NBR 12.648/11 (ABNT, 2011; 2016), respectivamente.

\subsection{Análises estatísticas}

Com o intuito de avaliar como as variáveis físicas, químicas, biológicas e ecotoxicológicas estão correlacionadas entre si, foi aplicada a Correlação de Spearman, utilizando o software Statistica versão 7.0 (2008), bem como a Análise dos Componentes Principais (ACP) com uso do software Past versão 2.16 (2001), a fim de identificar as variáveis mais relevantes em cada um dos períodos avaliados.

\section{RESULTADOS E DISCUSSÃO}

\subsection{Características físicas, químicas e biológicas}

A Tabela 1 apresenta os valores obtidos a partir da determinação das variáveis físicas, químicas e biológicas em amostras de água coletadas num trecho do RPS durante 1 ano (2013-2014). Os valores em destaque apresentam os resultados em desconformidade com a resolução CONAMA 357/05 para um corpo hídrico classe 2. 
A maioria das amostras de água (92,5\%) apresentou valores de $\mathrm{pH}$ dentro do estabelecido pelo CONAMA 357/05, porém observou-se que todos os valores de $\mathrm{pH}$ estiveram muito próximos de limite inferior. A condutividade, por sua vez, variou de 80 a $240 \mu \mathrm{S} \mathrm{cm}^{-1}$ em que a maioria dos valores obtidos foi igual ou inferior a $100 \mu \mathrm{S} \mathrm{cm}^{-1}$. De acordo com CETESB (2009), os valores acima de $100 \mu \mathrm{S} \mathrm{cm}^{-1}$ podem ser interpretados como decorrentes de concentrações elevadas de poluentes, visto que estes possuem muitos íons dissolvidos. A turbidez apresentou os maiores valores em fevereiro de 2014 com média de 4,73 UNT. Embora novembro tenha apresentado a maior precipitação, a média de turbidez foi de 0,37 UNT. Silva et al. (2008) também observaram valores de turbidez mais baixos no período chuvoso ao avaliarem a água do Rio Purus - AM. Os autores atribuíram os baixos valores de turbidez ao tamanho e natureza das partículas presentes na coluna d'água.

No presente estudo, as concentrações de OD variaram de 2,7 a 5,2 $\mathrm{mg} \mathrm{O}_{2} \mathrm{~L}^{-1}$, as quais, em sua maioria (95\%), estiveram abaixo do limite estabelecido pelo CONAMA 357/05 para um rio classe $2\left(>5,0 \mathrm{mg} \mathrm{O}_{2} \mathrm{~L}^{-1}\right.$ ). Diferentemente, os valores de DBO mantiveram-se dentro do limite $\left(<5,0 \mathrm{mg} \mathrm{O}_{2} \mathrm{~L}^{-1}\right)$, com valor máximo observado de 3,68 $\mathrm{mg} \mathrm{O}_{2} \mathrm{~L}^{-1}$.

Leandro et al. (2013), ao avaliar o mesmo trecho do RPS em agosto de 2012, encontrou valores de OD entre 5,0 e $6,4 \mathrm{mg} \mathrm{O}_{2} \mathrm{~L}^{-1}$ e obteve DBO média de $0,71 \mathrm{mg} \mathrm{O}_{2} \mathrm{~L}^{-1}$. Estes resultados evidenciam a piora da qualidade da água do rio ao longo dos anos. Os valores mais elevados de DBO observados no presente estudo podem ser atribuídos ao constante lançamento de efluentes e à redução da capacidade de diluição devido à baixa precipitação observada em agosto de 2013 e agosto de 2014, afetando diretamente a concentração de OD.

Dentre os períodos avaliados, o mês de novembro de 2013 apresentou maior DBO média $\left(2,75 \mathrm{mg} \mathrm{O}_{2} \mathrm{~L}^{-1}\right)$. Neste caso, além do lançamento de esgoto, a lixiviação de matéria orgânica causada pelas frequentes chuvas neste período pode ter contribuído para o aumento da DBO, considerando que a maioria dos pontos avaliados não possuía matas ciliares preservadas.

Os valores de DQO demonstram como os municípios envolvidos contribuem para poluição do RPS. Os menores valores de DQO foram encontrados à montante do trecho avaliado (APDM $\left.=6 \pm 3 \mathrm{mg} \mathrm{O}_{2} \mathrm{~L}^{-1}\right)$ e os maiores, à jusante $\left(\mathrm{CPTJ}_{\mathrm{J}}=12 \pm 4 \mathrm{mg} \mathrm{O}_{2} \mathrm{~L}^{-1}\right)$.

Ao longo do estudo, observou-se uma redução nos valores médios de DBO (42,4\%), ST $(31,1 \%)$ e DQO $(21,3 \%)$ quando comparados os meses de agosto de 2013 e agosto de 2014 . Esta redução pode estar associada à implantação de estações de tratamento de esgoto, no final do ano de 2013, nos municípios de Aparecida e Cachoeira Paulista, que até então não contavam com estes serviços. A implementação das ETE's pode ter contribuído para a redução destas variáveis, uma vez que efluentes, de modo geral, são particulados e ricos em matéria orgânica.

As concentrações de PT variaram de 19,4 a 40,5 $\mu \mathrm{g} \mathrm{L}^{-1}$, valores acima do limite máximo estabelecido pela Resolução CONAMA 357/05 $\left(<10 \mu \mathrm{g} \mathrm{L}^{-1}\right)$. As concentrações médias de NT mantiveram-se entre 18,1 e $27,6 \mathrm{mg} \mathrm{L}^{-1}$ em maio de 2014 e novembro de 2013, respectivamente. De acordo com Lima et al. (2016), os trechos do RPS com maior produtividade de nutrientes estão associados à maior proporção de áreas ocupadas pela agricultura e urbanização e à elevada densidade populacional. Diferentemente, a cobertura vegetal e baixa densidade demográfica resultam em trechos com baixo aporte de nutrientes. A partir dos valores anuais de PT e clorofila- $a$, calculou-se o grau de trofia do trecho avaliado, o qual foi classificado como mesotrófico, exceto pelo último ponto $\left(\mathrm{CPT}_{\mathrm{J}}\right)$, classificado como oligotrófico. Este ponto apresentava uma maior cobertura vegetal ciliar em relação aos demais pontos de coleta, o que pode ter influenciado no menor grau de trofia observado.

Como observado na Figura 1, o trecho avaliado apresenta, próximo às margens do rio, elevada taxa de urbanização e grande quantidade de áreas destinadas a lavouras e pastagens. Deste modo, o lançamento de efluentes domésticos e o arraste de nutrientes de áreas agrícolas contribuem significativamente para a produtividade de nutrientes do RPS. 
Tabela 1. Valores das variáveis físicas, químicas e biológicas de amostras de água do Rio Paraíba do Sul e limites estabelecidos pela Resolução CONAMA 357/05 para um corpo hídrico Classe 2.

\begin{tabular}{|c|c|c|c|c|c|c|c|c|c|c|c|c|c|}
\hline & Pontos & $\mathrm{pH}$ & $\begin{array}{c}\text { Temper. } \\
{ }^{\circ} \mathrm{C}\end{array}$ & $\begin{array}{c}\mathrm{CE} \\
\mu \mathrm{S} . \mathrm{cm}^{-1} \\
\end{array}$ & $\begin{array}{c}\text { Turbidez } \\
\text { UNT }\end{array}$ & $\begin{array}{c}\mathrm{OD} \\
\mathrm{mg} . \mathrm{L}^{-1}\end{array}$ & $\begin{array}{l}\text { DBO } \\
\text { mg.L } L^{-1}\end{array}$ & $\begin{array}{l}\text { DQO } \\
\text { mg.L-1 }\end{array}$ & $\begin{array}{c}\text { PT } \\
\mu \mathrm{g} . \mathrm{L}^{-1}\end{array}$ & $\begin{array}{c}\mathrm{NT} \\
\mathrm{mg} \cdot \mathrm{L}^{-1}\end{array}$ & $\begin{array}{l}\text { Clo- } a \\
\mu \mathrm{g} . \mathrm{L}^{-1} \\
\end{array}$ & $\begin{array}{c}\mathrm{ST} \\
\mathrm{mg} \cdot \mathrm{L}^{-1}\end{array}$ & $\begin{array}{c}\mathrm{CT} \\
\text { NMP. } 100 \mathrm{~mL}^{-1}\end{array}$ \\
\hline \multirow{8}{*}{ 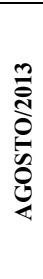 } & $\mathrm{APD}_{\mathrm{M}}$ & 6,2 & 21,6 & 90 & 0,53 & 4,63 & 1,88 & 2,81 & 27,1 & 22,5 & 1,58 & 104,0 & $2,810^{3}$ \\
\hline & $\mathrm{APD}_{\mathrm{J}}$ & 6,2 & 22,7 & 90 & 0,23 & 4,45 & 3,68 & 4,24 & 31,2 & 23,5 & 0,45 & 142,7 & $>1,610^{5}$ \\
\hline & GUAM $_{M}$ & 6,2 & 22,4 & 80 & 0,10 & 4,15 & 3,08 & 11,14 & 28,8 & 22,5 & 0,42 & 115,0 & $5,410^{4}$ \\
\hline & GUA $_{J}$ & 6,3 & 22,7 & 90 & 0,22 & 4,23 & 3,28 & 18,76 & 28,6 & 29,0 & 1,08 & 125,0 & $1,710^{4}$ \\
\hline & $\mathrm{LOR}_{\mathrm{M}}$ & 6,6 & 23,9 & 170 & 0,10 & 4,67 & 3,16 & 14,24 & 25,0 & 32,0 & 1,86 & 121,0 & $4,610^{3}$ \\
\hline & $\mathrm{LOR}_{\mathrm{J}}$ & 6,6 & 22,4 & 100 & 0,09 & 4,25 & 1,78 & 13,05 & 25,8 & 31,0 & 0,69 & 141,0 & $5,410^{4}$ \\
\hline & $\mathrm{CPT}_{\mathrm{M}}$ & 6,4 & 21,6 & 90 & 0,27 & 3,93 & 2,14 & 13,05 & 41,6 & 26,0 & 3,00 & 120,3 & $1,710^{3}$ \\
\hline & $\mathrm{CPT}_{\mathrm{J}}$ & 6,5 & 21,7 & 90 & 0,12 & 4,37 & 1,37 & 14,95 & 29,1 & 18,5 & 1,12 & 114,3 & $3,310^{3}$ \\
\hline \multirow{8}{*}{ 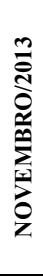 } & $\mathrm{APD}_{\mathrm{M}}$ & 6,6 & 24,0 & 90 & 0,11 & 4,20 & 2,25 & 2,65 & 29,0 & 23,0 & 5,29 & 221,0 & ND \\
\hline & $\mathrm{APD}_{\mathrm{J}}$ & 6,5 & 24,0 & 90 & 0,19 & 3,90 & 3,67 & 9,48 & 31,9 & 24,5 & 5,27 & 115,3 & $2,810^{3}$ \\
\hline & $\mathrm{GUA}_{\mathrm{M}}$ & 6,6 & 23,8 & 80 & 0,14 & 4,20 & 3,62 & 12,81 & 26,2 & 32,0 & 6,38 & 106,7 & ND \\
\hline & GUAJ & 6,4 & 23,9 & 80 & 0,54 & 4,23 & 2,89 & 4,24 & 26,4 & 38,5 & 7,57 & 208,7 & $4,910^{3}$ \\
\hline & $\mathrm{LOR}_{\mathrm{M}}$ & 6,7 & 24,3 & 90 & 0,21 & 4,23 & 3,67 & 15,03 & 28,4 & 41,5 & 7,76 & 128,7 & $3,510^{2}$ \\
\hline & $\mathrm{LOR}_{\mathrm{J}}$ & 6,2 & 23,7 & 70 & 0,23 & 4,20 & 1,85 & 13,44 & 29,1 & 26,0 & 6,76 & 185,3 & ND \\
\hline & $\mathrm{CPT}_{\mathrm{M}}$ & 6,8 & 24,1 & 90 & 0,58 & 3,67 & 2,13 & 21,62 & 32,4 & 18,5 & 8,25 & 143,3 & $2,810^{3}$ \\
\hline & $\mathrm{CPT}_{\mathrm{J}}$ & 6,6 & 23,9 & 240 & 0,95 & 4,35 & 1,93 & 16,46 & 30,4 & 17,0 & 5,80 & 116,0 & ND \\
\hline \multirow{8}{*}{ 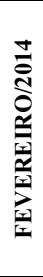 } & $\mathrm{APD}_{\mathrm{M}}$ & 6,5 & 28,4 & 100 & 3,97 & 3,87 & 1,28 & 5,83 & 25,9 & 17,0 & 1,72 & 106,7 & $7,910^{2}$ \\
\hline & $\mathrm{APD}_{\mathrm{J}}$ & 6,2 & 27,9 & 70 & 4,80 & 3,15 & 2,38 & 7,57 & 23,8 & 18,0 & 2,05 & 124,3 & $>1,610^{5}$ \\
\hline & $\mathrm{GUA}_{\mathrm{M}}$ & 6,1 & 27,9 & 70 & 7,30 & 2,67 & 2,08 & 8,68 & 24,1 & 25,0 & 8,32 & 118,0 & $2,810^{3}$ \\
\hline & GUA $_{J}$ & 6,6 & 28,9 & 80 & 4,70 & 2,87 & 1,22 & 7,25 & 24,8 & 25,5 & 1,92 & 144,3 & $1,310^{4}$ \\
\hline & $\mathrm{LOR}_{\mathrm{M}}$ & 6,4 & 27,7 & 80 & 3,90 & 3,67 & 2,78 & 6,14 & 22,6 & 35,5 & 2,27 & 123,3 & $4,910^{3}$ \\
\hline & $\mathrm{LOR}_{\mathrm{J}}$ & 6,3 & 27,8 & 80 & 2,50 & 3,77 & 2,47 & 3,92 & 22,8 & 22,5 & 2,61 & 145,7 & $2,310^{3}$ \\
\hline & $\mathrm{CPT}_{\mathrm{M}}$ & 6,7 & 27,5 & 150 & 5,77 & 3,95 & 2,35 & 7,89 & 30,3 & 21,0 & 22,03 & 134,7 & $2,810^{3}$ \\
\hline & $\mathrm{CPT}_{\mathrm{J}}$ & 6,5 & 27,4 & 90 & 4,90 & 3,50 & 2,98 & 5,67 & 25,7 & 19,5 & 3,11 & 123,3 & $1,710^{3}$ \\
\hline \multirow{8}{*}{ 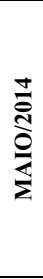 } & $\mathrm{APD}_{\mathrm{M}}$ & 6,6 & 20,9 & 70 & 2,10 & 5,20 & 0,93 & 11,62 & 20,0 & 16,0 & 0,34 & 72,0 & ND \\
\hline & $\mathrm{APD}_{\mathrm{J}}$ & 5,9 & 20,7 & 70 & 1,76 & 4,80 & 2,05 & 4,08 & 20,7 & 19,5 & 0,78 & 95,7 & $1,710^{2}$ \\
\hline & GUAM $_{M}$ & 6,2 & 21,0 & 70 & 1,92 & 4,40 & 1,57 & 10,27 & 20,4 & 26,5 & 0,58 & 74,3 & $9,210^{0}$ \\
\hline & GUA $_{J}$ & 6,2 & 20,8 & 70 & 2,50 & 4,70 & 1,75 & 12,49 & 24,1 & 28,5 & 1,37 & 100,0 & $4,610^{2}$ \\
\hline & $\mathrm{LOR}_{\mathrm{M}}$ & 6,8 & 21,1 & 80 & 2,40 & 4,87 & 3,27 & 19,79 & 23,4 & 32,0 & 0,34 & 99,7 & ND \\
\hline & $\mathrm{LOR}_{\mathrm{J}}$ & 5,9 & 20,8 & 70 & 1,67 & 4,75 & 0,88 & 13,29 & 23,6 & 11,0 & 0,23 & 87,7 & ND \\
\hline & $\mathrm{CPT}_{\mathrm{M}}$ & 6,1 & 21,5 & 80 & 2,30 & 4,45 & 1,34 & 12,02 & 23,0 & 7,0 & 0,18 & 87,7 & ND \\
\hline & $\mathrm{CPT}_{\mathrm{J}}$ & 6,0 & 21,1 & 80 & 1,59 & 5,00 & 2,20 & 13,76 & 24,5 & 4,5 & 0,28 & 114,7 & ND \\
\hline \multirow{8}{*}{ 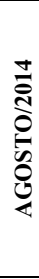 } & $\mathrm{APDM}_{\mathrm{M}}$ & 6,0 & 20,2 & 80 & 0,59 & 4,50 & 1,20 & 6,14 & 46,5 & 21,0 & 3,09 & 73,0 & $1,110^{4}$ \\
\hline & $\mathrm{APD}_{\mathrm{J}}$ & 6,5 & 20,4 & 110 & 0,62 & 4,37 & 2,30 & 7,81 & 50,0 & 27,5 & 3,10 & 88,7 & $1,610^{5}$ \\
\hline & GUA $_{M}$ & 6,7 & 20,2 & 190 & 0,46 & 3,90 & 1,80 & 11,38 & 47,3 & 21,5 & 3,33 & 82,0 & $5,410^{4}$ \\
\hline & GUAJ & 6,6 & 20,5 & 90 & 0,60 & 4,23 & 0,93 & 12,97 & 46,8 & 22,5 & 2,04 & 84,0 & $9,210^{4}$ \\
\hline & $\mathrm{LOR}_{\mathrm{M}}$ & 6,2 & 20,2 & 80 & 0,45 & 4,45 & 2,28 & 9,24 & 45,5 & 37,0 & 3,67 & 83,7 & $7,910^{3}$ \\
\hline & $\mathrm{LOR}_{\mathrm{J}}$ & 6,6 & 20,4 & 80 & 0,34 & 4,57 & 1,00 & 8,21 & 46,3 & 30,0 & 3,84 & 97,7 & $1,310^{4}$ \\
\hline & $\mathrm{CPT}_{\mathrm{M}}$ & 5,9 & 20,1 & 80 & 0,43 & 3,87 & 0,76 & 9,24 & 47,6 & 29,5 & 1,42 & 83,0 & $9,210^{4}$ \\
\hline & $\mathrm{CPT}_{\mathrm{J}}$ & 6,1 & 20,1 & 80 & 0,55 & 4,60 & 1,50 & 7,57 & 47,4 & 17,5 & 0,50 & 85,3 & $3,510^{4}$ \\
\hline \multicolumn{2}{|c|}{$\begin{array}{c}\text { CONAMA } \\
357 / 05\end{array}$} & $\begin{array}{c}6<\underset{9 H}{9}< \\
9\end{array}$ & - & - & $\begin{array}{l}<40 \\
\text { UNT }\end{array}$ & $\begin{array}{l}>5,00 \\
\operatorname{mg~L}^{-1}\end{array}$ & $\begin{array}{l}<5,00 \\
\operatorname{mg~L^{-1}}\end{array}$ & - & $\begin{array}{c}<10 \\
\mu \mathrm{g} \mathrm{L} L^{-1}\end{array}$ & - & $\begin{array}{c}<30 \\
\mu \mathrm{g} \mathrm{L^{-1 }}\end{array}$ & - & $\begin{array}{c}1000 \\
\text { NMP } \\
100 \mathrm{~mL}^{-1}\end{array}$ \\
\hline
\end{tabular}

O limite de CT estabelecido pela Resolução CONAMA 357/05 (1000 NMP.100 mL ${ }^{-1}$ ) foi excedido em $83 \%$ das amostras, o que demonstra forte lançamento de efluentes domésticos sem tratamento adequado. Vasconcellos et al. (2006), ao determinarem a presença de CT nas águas do Rio São Lourenço-RS, obtiveram $80 \%$ das amostras com valores acima do estabelecido, permitindo que os autores classificassem estas águas como impróprias para o consumo humano. Além disso, valores tão elevados podem tornar o tratamento desta água caro, devido a necessidade de etapas adicionais para total remoção destes micro-organismos. De acordo com ANA (2012), os municípios paulistas de Taubaté e Aparecida apresentam os maiores valores de CT do RPS. Valores elevados podem ser relacionados à poluição por efluentes domésticos sem tratamento adequado. Em alguns pontos do trecho avaliado foi observado este tipo de fonte poluidora de forma pontual. O ponto APDJ, localizado próximo a uma dessas fontes pontuais, mostrou-se positivo para a presença de CT durante todo o estudo, apresentando concentrações muito elevadas. Estes resultados reforçam a importância da implantação de ETE's no Rio Paraíba do Sul para garantia da qualidade da água para o abastecimento público. 


\subsection{Efeito tóxico agudo e crônico}

Do total de 40 amostras avaliadas neste estudo, 22,5\% apresentaram ETC utilizando a alga $R$. subcapitata e 5\% apresentaram ETA agudo para D. similis. O mês de novembro de 2013 apresentou ETA e ETC, diferente do mês de fevereiro de 2014, no qual foi observado apenas ETC (Tabela 2).

Tabela 2. Resultados dos bioensaios para determinação de efeito tóxico agudo (ETA) com $D$. similis e efeito tóxico crônico (ETC) com $R$. subcapitata a partir das amostras de água do Rio Paraíba do Sul, coletadas trimestralmente durante o período de agosto de 2013 e agosto de 2014.

\begin{tabular}{|c|c|c|c|c|c|c|c|c|c|c|}
\hline & \multicolumn{2}{|c|}{ Ago/13 } & \multicolumn{2}{|c|}{ Nov/13 } & \multicolumn{2}{|c|}{$\mathrm{Fev} / 14$} & \multicolumn{2}{|c|}{ Mai/14 } & \multicolumn{2}{|c|}{ Ago/14 } \\
\hline Pontos & ETA & ETC & ETA & ETC & ETA & ETC & ETA & ETC & ETA & ETC \\
\hline $\mathrm{APD}_{\mathrm{M}}$ & - & - & TOX & - & - & TOX & - & - & - & - \\
\hline $\mathrm{APD}_{\mathrm{J}}$ & - & - & - & TOX & - & - & - & - & - & - \\
\hline $\mathrm{GUA}_{\mathrm{M}}$ & - & - & TOX & TOX & - & - & - & - & - & - \\
\hline $\mathrm{GUA}_{J}$ & - & - & - & - & - & TOX & - & - & - & - \\
\hline $\mathrm{LOR}_{\mathrm{M}}$ & - & - & - & - & - & TOX & - & - & - & - \\
\hline $\mathrm{LOR}_{\mathrm{J}}$ & - & - & - & TOX & - & - & - & - & - & - \\
\hline $\mathrm{CPT}_{\mathrm{M}}$ & - & - & - & TOX & - & TOX & - & - & - & - \\
\hline $\mathrm{CPT}_{\mathrm{J}}$ & - & - & - & - & - & TOX & - & - & - & - \\
\hline
\end{tabular}

Nota: TOX: Efeito Tóxico Observado.

Bioensaios realizados a partir de amostras de água do RPS, utilizando Ceriodaphnia dubia, no ano de 2013, não apresentaram ETA. Porém, em 16\% das amostras foi detectado ETC (CETESB, 2009).

Em estudos de fitotoxicidade com Allium cepa realizados nos municípios de Tremembé e Aparecida nos anos de 2005 e 2006, as amostras de água do RPS promoveram o decréscimo no comprimento das raízes e a redução do índice mitótico (Barbério et al., 2009). Em testes de genotoxicidade utilizando $A$. серa para avaliar a água coletada no município de Tremembé em 2008, foram observadas anomalias cromossômicas nas células tratadas com a água coletada no período chuvoso (Oliveira et al., 2011).

No presente estudo, o efeito tóxico foi observado somente no período de maior precipitação. A maioria dos pontos analisados não possui matas ciliares às margens do RPS, e em alguns casos apresentam regiões impermeáveis. Solos sem cobertura vegetal estão sujeitos ao deslocamento de poluentes tóxicos para os rios por meio da lixiviação. Neste caso, a presença de vegetação próxima às margens do rio oferece proteção contra estes contaminantes (Coelho-Souza et al., 2007; Randhir et al., 2013). A degradação da vegetação promovida pela urbanização e atividades agrícolas pode ter favorecido o deslocamento de compostos tóxicos para o rio durante o período chuvoso, resultando no efeito tóxico observado.

\subsection{Análises estatísticas}

A partir da análise de Correlação de Spearman foi observada correlação negativa significativa $(p<0,05)$ entre a precipitação e o OD $(-0,402)$. Por outro lado, correlações positivas significativas $(\mathrm{p}<0,05)$ foram observadas entre a precipitação e temperatura $(0,570)$, ETC $(0,550)$, ETA $(0,324)$, ST $(0,394)$ e clorofila-a $(0,562)$. Os resultados apresentados reforçam a relação entre índice pluviométrico, erosão e consequente lixiviação de compostos tóxicos e matéria orgânica para o rio. Estes eventos podem ser observados a partir da redução 
de OD, pelo aumento de ST e pelo ETA e ETC observado nos períodos de maior precipitação.

Os períodos de coleta foram avaliados utilizando a ACP. A variância acumulada foi explicada em $66,53 \%$, sendo que o primeiro componente contribuiu com $28,9 \%$, o segundo com $15,9 \%$, o terceiro com $11,6 \%$ e o quarto com $10,1 \%$. O número de componentes foi determinado pela regra de Kaiser, na qual o último componente significativo dever ser aquele cujo autovalor é igual ou superior à média dos demais autovalores.

Os parâmetros temperatura $(0,873)$, precipitação $(0,746)$, ETC $(0,716)$, clorofila- $a(0,663)$ e ST $(0,661)$ apresentaram os maiores valores de correlação no componente 1 , que apresentou maior variância explicada $(28,99 \%)$. Em oposição, o menor valor corresponde ao OD $(-0,669)$. Neste caso, também é possível relacionar o período chuvoso (novembro de 2013 e fevereiro de 2014) ao arraste de compostos tóxicos e matéria orgânica para o corpo hídrico, resultando no efeito tóxico observado e na redução da solubilidade do OD.

Pela análise dos demais componentes selecionados, os parâmetros mais significativos foram DQO $(0,616)$, PT $(0,581)$, NT $(0,462), \mathrm{pH}(0,556)$ e condutividade $(0,509)$. Deste modo, é possível associar estas variáveis à poluição causada pelo lançamento de efluentes domésticos e industriais. Este tipo de impacto é muito comum sobre os rios da bacia do RPS, uma vez que a região é altamente industrializada e apresenta elevada densidade populacional.

Observou-se no gráfico da ACP um distanciamento entre os períodos com elevada precipitação (novembro de 2013 e fevereiro de 2014) e os demais períodos (Figura 3). Este distanciamento se deve às diferentes características conferidas ao rio pela ação das chuvas.

A distribuição espacial e temporal das chuvas é um fator que influencia fortemente os aspectos físicos, biológicos e socioeconômicos da região da bacia do RPS (Alvarenga et al., 2012). A chuva é um evento que altera as características de um corpo hídrico, bem como do ambiente ao seu redor. As variações pluviométricas ao longo do ano geram diferentes tipos de padrões sazonais que alteram a intensidade dos processos ambientais. Processos erosivos são desencadeados levando à lixiviação de matéria orgânica, nutrientes e poluentes. Além disso, as chuvas modificam o regime de vazão de corpos hídricos, aumentando a capacidade de diluição destes compostos (Santos et al., 2010; Geraldes e George, 2012). Neste contexto, observa-se que o período chuvoso surge como um agente intensificador dos impactos causados no RPS.

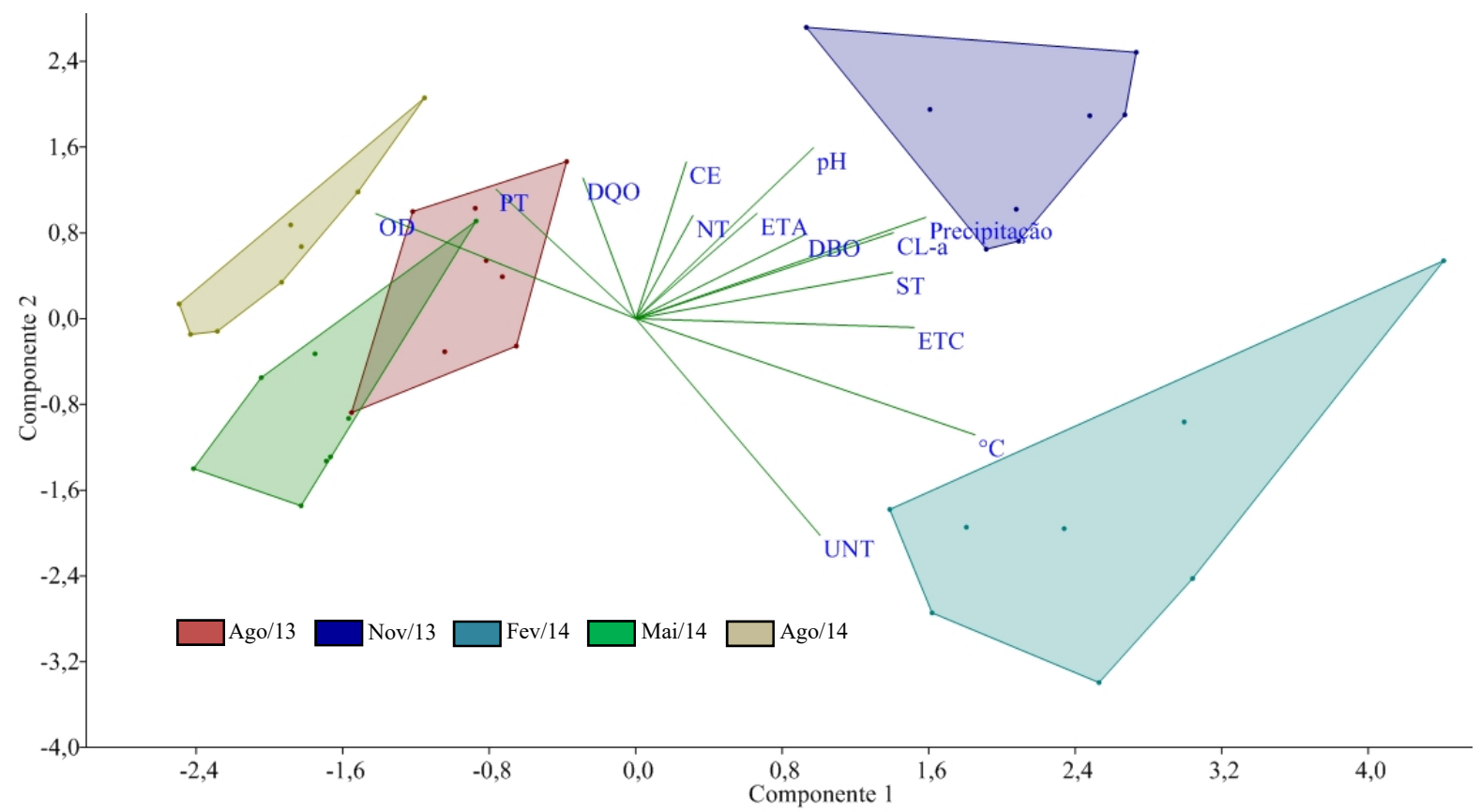

Figura 3. Análise dos Componentes Principais (ACP) das variáveis físicas, químicas e ecotoxicológicas do Rio Paraíba do Sul, em diferentes períodos do ano. 


\section{CONCLUSÕES}

Os baixos níveis de OD, as elevadas concentrações de PT e a presença de CT evidenciaram os impactos causados pelo lançamento de esgotos domésticos sobre o Rio Paraíba do Sul e demonstraram a necessidade de se investir em ações para adequar a qualidade da água deste manancial às exigências estabelecidas pela Resolução CONAMA 357/05. Dentre as ações, destacam-se a implantação de ETE's, a proteção da cobertura vegetal ciliar e o planejamento sobre a ocupação do solo na bacia.

A ocorrência de ETA e ETC somente no período chuvoso demonstrou que a chuva promoveu o arraste de compostos tóxicos para a coluna d'água, podendo causar prejuízos à biota aquática. Outras variáveis também se mostraram influenciadas pelas chuvas, como a DBO, clorofila- $a$ e os ST que apresentaram correlação positiva com o aumento da precipitação, e o OD que obteve correlação negativa. Deste modo, os resultados obtidos neste trabalho demonstraram que as chuvas, além de alterar as características da água do Rio Paraíba do Sul, atuam como um agente intensificador de impactos.

\section{AGRADECIMENTOS}

À CAPES e ao FEHIDRO pelo apoio e suporte a esta pesquisa.

\section{REFERÊNCIAS}

AlvarengA, L. A.; MARTINS, M. P. P.; CUARTAS, L. A.; PENTEAdO, V. A.; ANDRADE, A. Estudo da qualidade e quantidade da água em microbacia, afluente do Rio Paraíba do Sul - São Paulo, após ações de preservação ambiental. Revista Ambiente \& Água, v. 7, n. 3, p. 228-240, 2012. http:/dx.doi.org/10.4136/ambi-agua.987

AGÊNCIA NACIONAL DE ÁGUAS - ANA (Brasil). Panorama da qualidade das águas superficiais do Brasil. Brasília, 2012.

AMERICAN PUBLIC HEALTH ASSOCIATION - APHA; AMERICAN WATER WORKS ASSOCIATION - AWWA; WATER POLLUTION CONTROL FEDERATION - WEF. Standard methods for the examination of water and wastewater analysis. $21 \mathrm{st}$ ed. Washington, 2005.

ASSOCIAÇÃO BRASILEIRAS DE NORMAS TÉCNICAS - ABNT. NBR 9898. Preservação e técnicas de amostragem de efluentes líquidos e corpos receptores. Rio de Janeiro, 1987.

ASSOCIAÇÃO BRASILEIRAS DE NORMAS TÉCNICAS - ABNT. NBR 12648. Ecotoxicologia aquática - Toxicidade crônica - Método com algas (Chlorophyceae). Rio de Janeiro, 2011.

ASSOCIAÇÃO BRASILEIRAS DE NORMAS TÉCNICAS - ABNT. NBR 12713. Ecotoxicologia aquática - Toxicidade aguda - Método de ensaio com Daphnia spp (Crustacea, Cladocera). Rio de Janeiro, 2016.

BARBÉRIO, A.; BARROS, L.; VOLTOLINI, J. C.; MELLO, M. L. S. Evaluation of the cytotoxic and genotoxic potencial of water from the River Paraíba do Sul, in Brazil, with the Allium cepa L. test. Brazilian Journal of Biology, v. 69, n. 3, p. 837-842, 2009. http://dx.doi.org/10.1590/S1519-69842009000400010 
CARVALHO, K. Q.; LIMA, S. B.; PASSIG, F. H.; GUSMÃO, L. K. SOUZA, D. C.; KREUTZ, C.; BELINI, A. D.; ARANTES, E. J. Influence of urban área on the water quality of the Campo River basin, Paraná State, Brazil. Brazilian Journal of Biology, v. 75, n. 4, p. 96-106, 2015. http://dx.doi.org/10.1590/1519-6984.00413suppl

COMITÊ DE INTEGRAÇÃO DA BACIA HIDROGRÁFICA DO RIO PARAÍBA DO SUL CEIVAP. Dados Geoambientais. Disponível em:

http://www.ceivap.org.br/geoambientais.php.Acessoem:20março2014

COELHO-SOUZA, S. A.; MIRANDA, M. R.; GUIMARÃES, J. R. D. The importance of aquatic macrophytes in mercury cycle in Tapajós river (PA). Oecologia Australis, v. 11, n. $2,2007$.

COMPANHIA DE TECNOLOGIA E SANEAMENTO AMBIENTAL - CETESB. Significado ambiental e sanitário das variáveis de qualidade das águas e dos sedimentos e metodologias analíticas e de amostragem. São Paulo, 2009.

CONSELHO NACIONAL DO MEIO AMBIENTE - CONAMA (Brasil). Resolução n³57, de 17 de março de 2005. Diário Oficial [da] União, Brasília, 2005.

DEMANBORO, A. C. Gestão ambiental e sustentabilidade na macrometrópole paulista - Bacia do Rio Paraíba do Sul. Sociedade e Natureza, v. 27, n. 3, p. 515-530, 2015.

GALVANI, F.; GAERTNER, E. Adequação da metodologia Kjeldahl para determinação de nitrogênio total e proteína bruta. Brasília: EMBRAPA, 2006.

GERALDES, A. M.; GEORGE, C. Limnological variations of a deep reservoir in periods with distinct rainfall patterns. Acta Limnologica Brasiliensis, v. 24, n. 4, p. 417-426, 2012.

LORENZEN, C. J. Determination of chlorophyll and pheopigments: spectrophotometric equations. Limnology and Oceonography, v. 12, p. 343-346, 1967. http://dx.doi.org/10.4319/lo.1967.12.2.0343

MARENGO, J. A.; ALVES, L. M. Tendências hidrológicas da Bacia do Rio Paraíba do Sul. Revista Brasileira de Meteorologia, v. 20, n. 2, p. 215-226, 2005.

MALAFAIA, J. P.; MIRANDA, A. C.; GOMES, H. P. A Bacia do Rio Paraíba do Sul: cenário de uma atividade de EA a partir de problemas ambientais. ANAP Brasil, v. 5, n. 5, p. 112, 2012. http://dx.doi.org/10.17271/19843240552012394

OLIVEIRA, L. M.; VOLTOLINI, J. C.; BARBÉRIO, A. Potencial mutagênico dos poluentes na água do Rio Paraíba do Sul em Tremembé, SP, Brasil, utilizando o teste Allium cepa. Revista Ambiente \& Água, v. 6, n. 1, 2011. http://dx.doi.org/10.4136/ambi-agua.176

PASSIG, F. H.; LIMA, S. B.; CARVALHO, K. Q.; HALMEMAN, M. C. R.; SOUZA, P. C.; GUSMÃO, L. K. Monitoring of urban and rural basins: water quality of Mourão basin. Brazilian Journal of Biology, v. 75, n. 4, p. 158-164, 2015. http://dx.doi.org/10.1590/1519-6984.01213suppl

RANDHIR, T. O.; EKNESS, P. Water quality change and habitat potential in riparian ecosystems. Ecohydrology \& Hidrobiology, v. 13, p. 192-200, 2013. http://dx.doi.org/10.1016/j.ecohyd.2013.09.001

SANTOS, G. G.; GRIEBELER, N. P.; OLIVEIRA, L. F. C. Chuvas intensas relacionadas à erosão hídrica. Revista Brasileira de Engenharia Agrícola e Ambiental, v. 14, n. 2, p. 115-123, 2010. http://dx.doi.org/10.1590/S1415-43662010000200001 
SILVA, A. E. P.; ANGELIS, C. F.; MACHADO, L. A. T.; WAICHAMAN, A. V. Influência da precipitação na qualidade da água do Rio Purus. Acta Amazonica, v. 38, n. 4, p. 733 742, 2008. http://dx.doi.org/10.1590/S0044-59672008000400017 


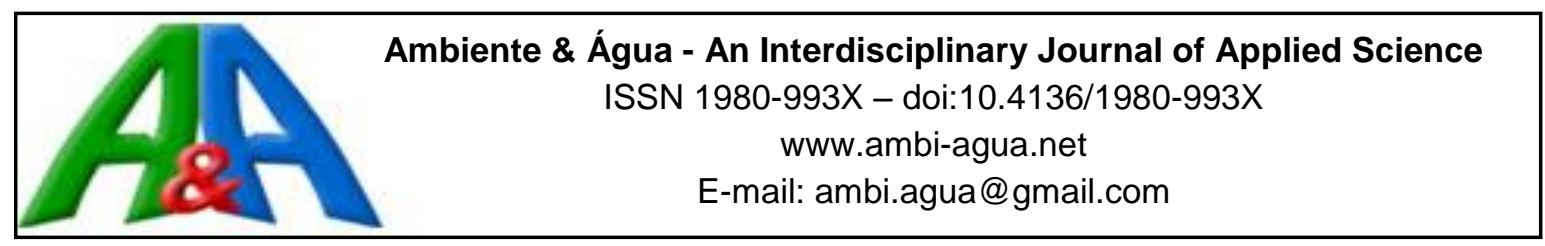

\title{
Ocorrência e remoção de estrogênios por processos de tratamento biológico de esgotos
}

\author{
doi:10.4136/ambi-agua.1992
}

Received: 24 Aug. 2016; Accepted: 20 Dec. 2016

\author{
Danieli Lima da Cunha ${ }^{1,2 *}$; Lícia Murito de Paula ${ }^{1}$; \\ Samuel Muylaert Camargo da Silva ${ }^{3}$; Daniele Maia Bila ${ }^{4}$; \\ Estefan Monteiro da Fonseca ${ }^{2}$; Jaime Lopes da Mota Oliveira ${ }^{1}$ \\ ${ }^{1}$ Fundação Oswaldo Cruz (FIOCRUZ), Rio de Janeiro, RJ, Brasil \\ Departamento de Saneamento e Saúde Ambiental \\ ${ }^{2}$ Universidade Federal Fluminense (UFF), Niterói, RJ, Brasil \\ Departamento de Geologia \\ ${ }^{3}$ Universidade do Estado do Rio de Janeiro (UERJ), Rio de Janeiro, RJ, Brasil \\ Programa de Pós-Graduação em Gestão e Regulação de Recursos Hídricos \\ ${ }^{4}$ Universidade do Estado do Rio de Janeiro (UERJ), Rio de Janeiro, RJ, Brasil \\ Departamento de Engenharia Sanitária e do Meio Ambiente \\ *Autor correspondente: e-mail: danielicunha@ hotmail.com, \\ liciamurito@gmail.com,samu_muylaert@hotmail.com, \\ danielebilauerj@gmail.com, oceano25@hotmail.com, \\ jaimelmoliveira@gmail.com
}

\section{RESUMO}

Os estrogênios são micropoluentes capazes de causar alterações no sistema endócrino de organismos aquáticos. Uma das principais fontes da sua introdução no meio hídrico é através do lançamento de esgotos domésticos. Este artigo apresenta uma revisão sobre a ocorrência dos estrogênios estrona, estradiol, estriol e etinilestradiol em esgotos brutos e tratados e em águas superficiais, bem como sobre sua remoção por diferentes processos de tratamento biológico de esgoto. Os poucos trabalhos realizados no Brasil mostram que a concentração desses compostos nestas matrizes é expressiva. Além disso, foi observado um decaimento nas concentrações desses estrogênios do esgoto bruto ao tratado. Os sistemas aeróbios, como lodos ativados, mostraram melhor desempenho na remoção de estrogênios, sobretudo por meio da sorção ao lodo. No entanto, pouco se sabe sobre os mecanismos de degradação de tais compostos nestes processos e sobre o comportamento de seus conjugados. Portanto, os processos de tratamento biológico de esgoto representam uma barreira à introdução de estrogênios nos corpos hídricos.

Palavras-chave: estrogênios em esgoto, remoção, tratamento biológico.

\section{Occurrence of estrogens and their removal by biological processes of sewage treatment}

\begin{abstract}
Estrogens are micropollutants that may harm aquatic organisms endocrine system. They enter water bodies mainly through domestic sewage. This paper aims to review the estrone, estradiol, estriol and ethinyl estradiol occurrence in untreated and treated sewage and surface water, as well as their removal efficiency through employment of different sewage biological
\end{abstract}


treatment methods. The few studies carried out in Brazil show a significant concentration of these compounds in the reviewed sources, with a downward trend from raw to treated sewage. Aerobic systems, such as activated sludge, showed better results at removing the estrogens, mainly through adsorption by sludge. However, little is known about the degradation mechanisms of such compounds and the behavior of their conjugates. Therefore, biological processes of sewage treatment represent a barrier to the introduction of estrogens to the water bodies.

Keywords: biological treatment, estrogens in the sewage, removal.

\section{INTRODUÇÃO}

Com o avanço da ciência e do conhecimento sobre os efeitos decorrentes da poluição dos corpos hídricos, alguns países da União Europeia e os Estados Unidos vêm buscando ampliar a regulamentação e o monitoramento de novos compostos, como os desreguladores endócrinos, que antes não eram objeto de atenção por parte dos dispositivos legais (Cunha et al., 2016).

Estudos mostram que essas substâncias, mesmo em concentrações extremamente baixas (ng $\mathrm{L}^{-1}$ ), podem promover alterações no sistema endócrino dos organismos aquáticos. Esses compostos são capazes de desencadear efeitos adversos como interferir no crescimento, desenvolvimento e/ou reprodução desses organismos (Barceló e Petrovic, 2008; Bila e Dezotti, 2007). Em face disto, a comunidade científica busca compreender melhor as vias de introdução e dispersão desses compostos no meio ambiente e os possíveis mecanismos de sua remoção. $\mathrm{O}$ maior entendimento da dinâmica destes compostos no meio ambiente permitirá o aperfeiçoamento das tecnologias de remoção através dos processos de tratamento de efluentes.

Dentre este grupo de substâncias, os estrogênios merecem especial atenção, uma vez que a sua presença em efluentes urbanos tem sido diretamente correlacionada a impactos à biota aquática. Estudos ecotoxicológicos apontam que a exposição contínua aos estrogênios está associada a alterações bioquímicas e histopatológicas, redução significativa na taxa de fertilização, diminuição na eclosão de ovos e modificações comportamentais no acasalamento em espécies de peixes, anfíbios, crustáceos e gastrópodes (Silva et al., 2012; Bergman et al., 2012; Giusti et al., 2014; Luna et al., 2015; Garmshausen et al., 2015). O efeito mais alarmante é o processo de feminização (desenvolvimento de características sexuais femininas em machos, incluindo anatomia reprodutiva feminina), uma vez que compromete o ciclo reprodutivo de toda uma população, podendo assim desencadear um desequilíbrio ecossistêmico (Gilbert, 2012).

Neste contexto de risco à biota aquática, o lançamento de esgoto "in natura" ou tratado tem sido indicado como principal contribuinte para a introdução de estrogênios no meio hídrico. Dessa forma, os processos de tratamento podem representar uma barreira para seu lançamento nos corpos hídricos. Porém, para que a depuração seja efetiva, é necessário implementar sistemas que possam realmente remover e/ou degradar tais compostos. No entanto, observa-se que os processos convencionais de tratamento de esgotos podem não remover integralmente esses compostos e, desta forma, conduzir a sua introdução contínua no meio hídrico (Brandt et al., 2013; Aquino et al., 2013).

Diante da crescente preocupação com a qualidade da água e a fim de minimizar os riscos causados pela poluição hídrica, países têm investido em estudos para o aprimoramento das tecnologias de tratamento de águas residuais. Portanto, este trabalho tem como objetivo apresentar uma revisão sobre a ocorrência dos estrogênios estrona, estriol, estradiol e etinilestradiol nas águas residuais e superficiais. Além disso, este artigo mostra a eficiência encontrada por alguns autores em relação à remoção desses compostos por processos biológicos para o tratamento de esgoto.

Rev. Ambient. Água vol. 12 n. 2 Taubaté - Mar. / Apr. 2017 


\section{CARACTERÍSTICAS FÍSICO-QUÍMICAS E METABÓLICAS}

Os estrogênios, bem como todos os hormônios esteroidais, possuem uma mesma estrutura química básica de 17 átomos de carbono dispostos em quatro anéis (A, B, C e D) ligados entre si. Configurações estruturais no anel $\mathrm{D}$ dão origem a diferentes estrogênios (Tabela 1). Na posição do carbono 17 (C17) a estrona possui uma carbonila enquanto o estradiol possui uma hidroxila, já o estriol possui duas hidroxilas, uma no C16 e a outra no C17.

O etinilestradiol difere-se do estradiol através de um único radical, o etinil, também no carbono 17. Esta diferença faz com que o etinilestradiol possua maior potencial estrogênico e também se torne mais resistente à degradação com relação ao estradiol (Combalbert e Hernandez-Raquet, 2010; WHO e IARC, 2007).

Tabela 1. Propriedades físico-químicas de alguns estrogênios (Hamid e Eskicioglu 2012).

Estrogênio

a CAS: Chemical Abstract Service. Número que corresponde à identificação química da substância.

b K ow: Coeficiente de partição octanol/água.

c $\mathrm{pK}_{\mathrm{a}}$ : Constante de dissociação ácida.

$\mathrm{d} \mathrm{K}_{\mathrm{d}}$ : Coeficiente de distribuição.

Com relação as propriedades físico-químicas, um parâmetro importante e frequentemente usado na descrição do comportamento de um contaminante no ambiente é o coeficiente de distribuição $\left(\mathrm{K}_{\mathrm{d}}\right)$. Este por sua vez está diretamente associado ao coeficiente de partição octanol/água $\left(\mathrm{K}_{\mathrm{ow}}\right)$.

$\mathrm{O} \mathrm{K}_{\mathrm{d}}$ é determinado pela razão entre a massa do composto na fase sólida e na fase líquida. Este parâmetro é muito útil para estimar o potencial de sorção do contaminante dissolvido em contato com o solo. Quanto maior o $\mathrm{K}_{\mathrm{d}}$, maior a tendência do contaminante ficar adsorvido ao solo ou sedimento (CETESB, 2001). 
Ainda com relação à mobilidade dos estrogênios, o $\mathrm{K}_{\mathrm{ow}}$, definido pela a relação entre concentração do contaminante orgânico na fase octanol e a concentração deste na fase aquosa, se apresenta como importante coeficiente para compreender a hidroafinidade destes compostos. Valores de $\log \mathrm{K}_{\text {ow }}$ para os estrogênios variam entre 2,45 e 4. Este resultado mostra que os mesmos são moderadamente hidrofóbicos, tendo a tendência de se associarem à fase sólida.

No que diz respeito às características metabólicas, a maioria dos estrogênios produzidos pelo corpo humano, ou mesmo aqueles que são ingeridos através de anticoncepcionais e/ou de medicamentos utilizados na terapia de reposição hormonal, são excretados pelas fezes e urina em sua forma conjugada (sulfato e/ou glicuronídeos) (Cano et al., 1997). Tais conjugados polares são biologicamente menos ativos e mais solúveis em água quando comparados aos estrogênios livres, não conjugados (D'ascenzo et al., 2003).

Vale observar que, mesmo com estes mecanismos de degradação, estudos constataram a ocorrência de estrogênios em sua forma livre em diferentes compartimentos ambientais, principalmente em afluentes e efluentes de ETE. Isso ocorre devido ao um processo natural, no qual as bactérias presentes no meio, principalmente a Escherichia coli, são capazes de desconjugar os estrogênios, tornando-os novamente a sua forma livre (Fent et al., 2006; D'ascenzo et al., 2003).

Este processo ocorre, pois, a $E$. coli é capaz de sintetizar uma grande quantidade da enzima $\beta$-glucuronisidase, responsável por essa transformação. A natureza recalcitrante dos conjugados sulfatos ao longo das ETEs pode ser explicada por uma atividade mais fraca da arilsulfatase da E. coli comparada com a $\beta$-galogsidade (Hamid e Eskicioglu 2012).

\section{OCORRÊNCIA DE ESTROGÊNIOS NO ESGOTO BRUTO, TRATADO E NA ÁGUA SUPERFICIAL}

Uma vez constatada a introdução contínua de estrogênios no meio hídrico e seus efeitos negativos à qualidade das águas e, consequentemente, à saúde dos ecossistemas, o monitoramento destas substâncias vem ganhando especial atenção. Assim, métodos têm sido desenvolvidos visando a determinação desses compostos nas mais variadas matrizes aquáticas.

A Figura 1 resume as concentrações de estrogênios relatadas por diversos estudos, que analisaram esgoto bruto, esgoto tratado e águas superficiais em diversos países, com exceção do Brasil.

Para construção do gráfico apresentado na Figura 1 foram consultados os dados obtidos de cerca de 15 artigos, conduzidos em diferentes países. Estes foram então introduzidos e processados no programa estatístico SPSS versão 22.0, optando-se por gerar um gráfico em formato boxplot. Na Figura 1, são apresentadas as barras principais, limitadas pelo primeiro e terceiro quartil; as medianas; e as hastes dos limites inferior e superior. Nesta representação, foram excluídos os pontos discrepantes (outliers).

No geral, as concentrações dos estrogênios no esgoto tratado foram menores do que as do esgoto bruto. Padrão este que pode ser visto mais claramente em relação aos estrogênios naturais (estrona, estradiol e estriol). Já com relação ao estrogênio sintético (etinilestradiol), este decaimento é menos evidente. Tal resultado reflete a ação de processos de tratamento de esgotos na remoção destes compostos.

Observa-se ainda que a matriz esgoto tratado foi a que se mostrou com maior variabilidade para os diferentes estrogênios estudados. Tal constatação pode ser explicada pela adoção de diferentes tecnologias de tratamento de efluentes, bem como pela vazão de entrada e carga orgânica aplicada, o que, por consequência, acarreta em uma heterogeneidade das eficiências de degradação/remoção de estrogênios. 


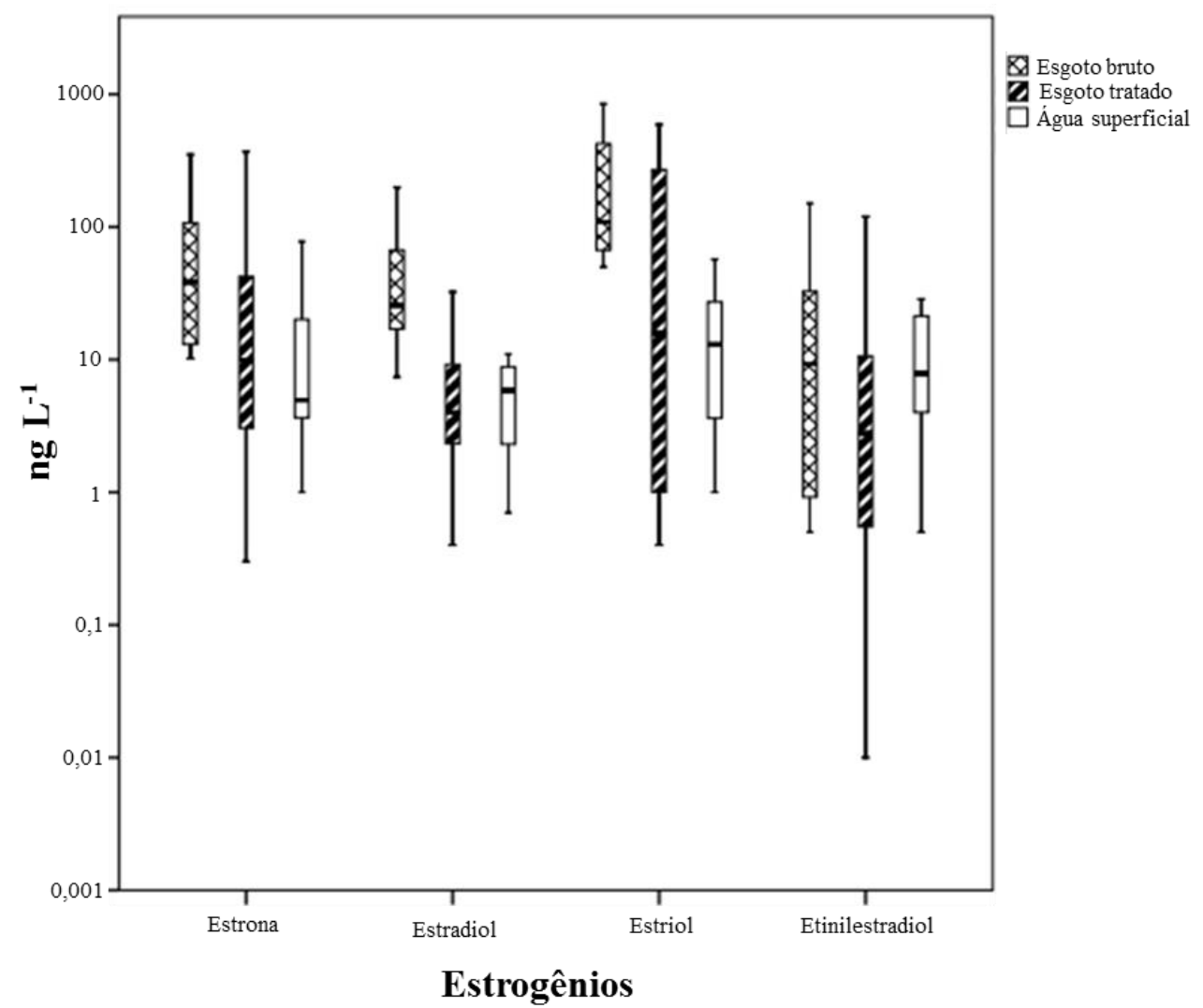

Figura 1. Variação das concentrações dos estrogênios estrona, estradiol, estriol e etinilestradiol no esgoto bruto, esgoto tratado e águas superficiais em diversos países (Drewes et al., 2005; Morteani et al., 2006; Chimchirian et al., 2007; Zorita et al., 2009; Duong et al., 2010; Zhang et al., 2011; Atkinson et al., 2012; Martin et al., 2012; Wang et al., 2012; Williams et al., 2012; Ye et al., 2012; Jin et al., 2013; Manickum e John, 2014; e Liu et al., 2015).

São ainda apresentados na sequência (Tabela 2) dados nacionais de concentrações de estrogênios encontrados nestas mesmas matrizes.

Embora tenham sido encontrados poucos artigos brasileiros sobre a ocorrência de estrogênios nas matrizes aquáticas, eles mostram que as concentrações destes compostos, com exceção do estriol (sobre o qual não foram encontrados estudos), foram bem superiores quando comparados aos dados relatados pelos estudos internacionais. Enquanto as concentrações destes estrogênios nos estudos internacionais não ultrapassaram a ordem de $1.000 \mathrm{ng} \mathrm{L}^{-1}$, no Brasil chegaram a $5.000 \mathrm{ng} \mathrm{L}^{-1} \mathrm{em}$ esgoto bruto, e até $4.000 \mathrm{ng} \mathrm{L}^{-1} \mathrm{em}$ águas superficiais (Tabela 2).

Outro ponto que merece atenção dentre os estudos de âmbito nacional, é ampla dispersão das concentrações, nas três matrizes estudadas. Os diferentes autores detectaram concentrações em ordens de grandeza bem distintas entre si, o que dificulta o estabelecimento de concentrações médias desses compostos para cada uma das matrizes. Tais variações podem ser explicadas por diversos fatores, como: condições locais, diferenças sociais e de saúde pública, e, ainda, diferenças entre as metodologias aplicadas para a condução de cada um dos estudos (Xu et al., 2012).

Mesmo com tal variabilidade entre os dados, é possível observar que ao analisar as concentrações obtidas por um mesmo autor, pode-se notar que há um significativo decaimento das concentrações desses estrogênios do esgoto bruto ao tratado (Pessoa et al., 2014; Queiroz et al., 2012; Froehner et al., 2011; Ternes et al., 1999), padrão este que confere com aquele 
observado nos estudos internacionais (Atkinson et al., 2012; Chimchirian et al., 2007; Duong et al., 2010; Manickum e John, 2014; Martin et al., 2012; Zhang et al., 2011; Zorita et al., 2009).

Tabela 2. Concentrações de estrogênios encontrados em esgoto bruto, esgoto tratado e águas superficiais do Brasil.

\begin{tabular}{|c|c|c|c|c|}
\hline Estrogênio & Matriz & Local & oncentração ng L ${ }^{-1}$ & Referência \\
\hline \multirow{7}{*}{ 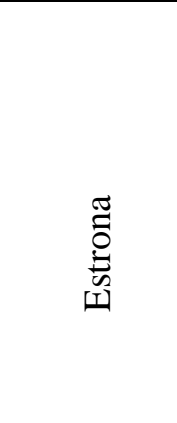 } & \multirow{3}{*}{$\begin{array}{l}\text { Esgoto } \\
\text { bruto }\end{array}$} & Curitiba & $870-1.380$ & Froehner et al. (2011) \\
\hline & & Fortaleza & 3.050 & Pessoa et al. (2014) \\
\hline & & Rio de Janeiro & 40 & Ternes et al. (1999) \\
\hline & \multirow{3}{*}{$\begin{array}{l}\text { Esgoto } \\
\text { tratado }\end{array}$} & Curitiba & $<\mathrm{LD}$ & Froehner et al. (2011) \\
\hline & & Fortaleza & 2.080 & Pessoa et al. (2014) \\
\hline & & Rio de Janeiro & 7 & Ternes et al. (1999) \\
\hline & $\begin{array}{l}\text { Água } \\
\text { superficial }\end{array}$ & $\begin{array}{l}\text { Atibaia, Campinas, } \\
\text { Paulínia e Americana }\end{array}$ & $<16$ & Montagner e Jardim (2011) \\
\hline \multirow{11}{*}{ 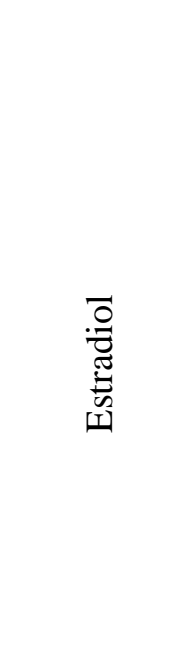 } & \multirow{4}{*}{$\begin{array}{l}\text { Esgoto } \\
\text { bruto }\end{array}$} & Belo Horizonte & $<9,3-31$ & Queiroz et al. (2012) \\
\hline & & Curitiba & $1.330-2.270$ & Froehner et al. (2011) \\
\hline & & Fortaleza & 776 & Pessoa et al. (2014) \\
\hline & & Rio de Janeiro & 21 & Ternes et al. (1999) \\
\hline & \multirow{4}{*}{$\begin{array}{l}\text { Esgoto } \\
\text { tratado }\end{array}$} & Belo Horizonte & $<9,3$ & Queiroz et al. (2012) \\
\hline & & Curitiba & $490-760$ & Froehner et al. (2011) \\
\hline & & Fortaleza & 397 & Pessoa et al. (2014) \\
\hline & & Rio de Janeiro & 1 & Ternes et al. (1999) \\
\hline & \multirow{3}{*}{$\begin{array}{l}\text { Água } \\
\text { superficial }\end{array}$} & $\begin{array}{l}\text { Atibaia, Campinas, } \\
\text { Paulínia e Americana }\end{array}$ & $<45-6.806$ & Montagner e Jardim (2011) \\
\hline & & Belo Horizonte & $<1-37$ & Moreira et al. (2009) \\
\hline & & $\begin{array}{l}\text { Ouro Preto, Itabirito } \\
\text { Rio Acima, Nova Lima }\end{array}$ & $<4-63$ & Moreira et al. (2011) \\
\hline \multirow{9}{*}{ 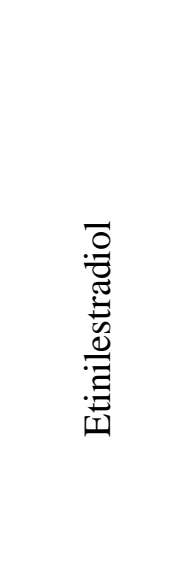 } & \multirow{3}{*}{$\begin{array}{l}\text { Esgoto } \\
\text { bruto }\end{array}$} & Belo Horizonte & $<12,4-41,3$ & Queiroz et al. (2012) \\
\hline & & Curitiba & $600-1.260$ & Froehner et al. (2011) \\
\hline & & Fortaleza & 3.180 & Pessoa et al. (2014) \\
\hline & \multirow{3}{*}{$\begin{array}{l}\text { Esgoto } \\
\text { tratado }\end{array}$} & Belo Horizonte & $<12,4$ & Queiroz et al. (2012) \\
\hline & & Curitiba & $<$ LD. -470 & Froehner et al. (2011) \\
\hline & & Fortaleza & 176 & Pessoa et al. (2014) \\
\hline & \multirow{3}{*}{$\begin{array}{l}\text { Água } \\
\text { superficial }\end{array}$} & $\begin{array}{l}\text { Atibaia, Campinas, } \\
\text { Paulínia e Americana }\end{array}$ & $<17-4.390$ & Montagner e Jardim (2011) \\
\hline & & Belo Horizonte & $<1-54$ & Moreira et al. (2009) \\
\hline & & $\begin{array}{l}\text { Ouro Preto, Itabirito, } \\
\text { Rio Acima, Nova Lima }\end{array}$ & $<5-64$ & Moreira et al. (2011) \\
\hline
\end{tabular}

Nota: LD - Limite de detecção.

Após o processo de tratamento de esgoto e seu lançamento nos corpos hídricos, os estrogênios são ainda submetidos a diferentes graus de atenuação natural que podem minimizar o seu impacto ambiental, como por exemplo, a diluição, a eventual adsorção em sólidos em suspensão e em sedimentos, a fotólise e a biodegradação aeróbia (Gómez et al., 2012; Pal et al., 2010). Mas há ainda que se considerar a possibilidade de ressuspensão destes compostos para coluna d'água, o que pode torná-los novamente mais biodisponíveis. 
Com relação às águas superficiais, no geral, a estrona é o estrogênio mais abundante e frequentemente detectado (Manickum e John, 2014; Jin et al., 2013; Wang et al., 2012; Duong et al., 2010; Kim et al., 2007). A maior ocorrência desse estrogênio nos corpos hídricos devese principalmente ao fato da estrona ser o principal produto da biodegradação do estradiol (Figura 2), durante os processos de tratamento de esgoto (Zorita et al., 2009). Segundo Sodré et al. (2007), dentre os estrogênios naturais, o estradiol é o mais potente, sendo estrogenicamente doze vezes mais ativo que a estrona. No que diz respeito ao etinilestradiol, por se tratar de um estrogênio sintético, sua ocorrência no meio hídrico pode ser diretamente relacionada ao consumo do mesmo, através de contraceptivos orais.

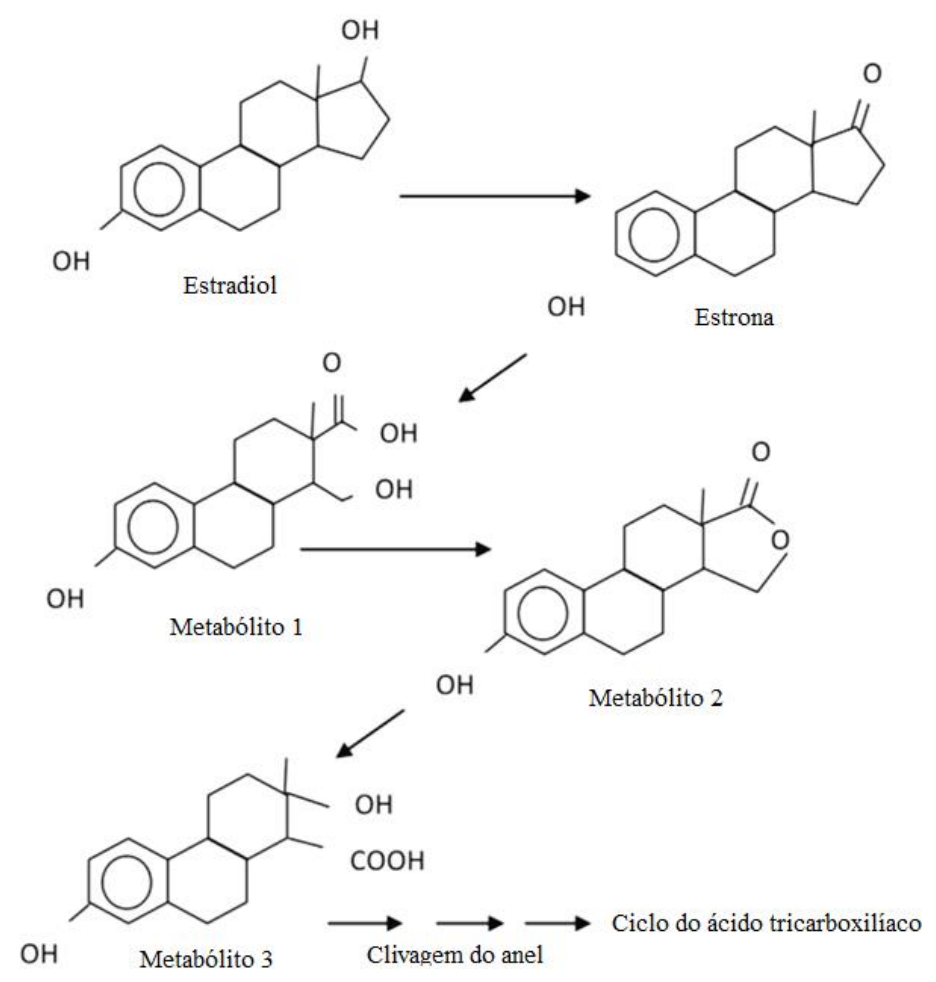

Figura 2. Via metabólica de degradação do estradiol por bactérias presentes no esgoto.

Fonte: Adaptado de Hamid e Eskicioglu (2012).

Caracterizar o comportamento dos estrogênios nas diferentes matrizes aquáticas apresenta-se como uma atividade complexa devido suas características físico-químicas, das propriedades do meio receptor e das inúmeras variáveis que atuam em conjunto no ambiente, como temperatura, turbidez, $\mathrm{pH}$, alcalinidade, oxigênio dissolvido, radiação, relações de sinergismo, atividade microbiana e concentração de outras substâncias. No entanto, o conhecimento desses compostos, seus níveis de ocorrência em matrizes ambientais, bem como a avaliação dos seus efeitos, configuram-se como atividades extremamente importantes (Bila e Dezotti, 2007).

\section{REMOÇÃO DE ESTROGÊNIOS POR PROCESSOS BIOLÓGICOS DE TRATAMENTO DE ESGOTO}

Os processos biológicos de tratamento são amplamente empregados no Brasil e no mundo. Dentre os processos de tratamento classificados como biológicos, o processo aeróbio por lodos ativados é o mais utilizado, seguido pelos sistemas anaeróbios, como o RAFA (Reator Anaeróbio de Fluxo Ascendente) - também conhecido como UASB "Upflow Anaerobic Sludge Blanket" - e as lagoas de estabilização. 
Atualmente, variantes destes diferentes processos foram e estão sendo desenvolvidos para melhorar a remoção de matéria orgânica e nutrientes (nitrogênio e fósforo). Por outro lado, os estudos envolvendo a remoção dos micropoluentes emergentes, como os estrogênios, partem da análise do potencial de aplicação destas variantes com tal finalidade.

Para comparar a eficiência da remoção de estrogênios, além das variações dos parâmetros de controle de processo de lodo ativado, como: tempo de retenção dos sólidos (TRS), tempo de detenção hidráulica (TDH), índice volumétrico do lodo, taxa de aeração, remoção de matéria orgânica (DBO/DQO), remoção de nitrogênio e fósforo; também são simuladas variações nos tipos de processos por lodos ativados, tais como: valos de oxidação, reator em batelada sequencial, processo anóxico/aeróbio, lodo ativado utilizando clarificador no final do processo, lodos ativados com posterior tratamento terciário e bactérias anaeróbias facultativas redutoras de ferro (Petrie et al., 2014; Shi et al., 2013; Kumar et al., 2011; Ivanov et., 2010; Hashimoto e Murakami, 2009).

Hashimoto e Murakami (2009) compararam as eficiências de remoção de estrogênios por lodos ativados convencional e por lodos ativados com valos de oxidação com variação do TRS. Esses autores observaram que o processo por lodos ativados convencional foi capaz de degradar praticamente todo o estradiol (mais de 95\%) à estrona com apenas cinco minutos de processo, em escala laboratorial; e a estrona, por sua vez, permaneceu posteriormente sorvida ao lodo após 24 horas de detenção hidráulica e pôde ser removida por sedimentação. Por outro lado, nos ensaios em que foi utilizado o lodo dos valos de oxidação, somente houve remoção pela sorção após uma hora de processo. No estágio anóxico do valo de oxidação, a remoção tanto da estrona quanto do estradiol foi menor (cerca de 20\%). Em ambos os processos, o etinilestradiol teve alta taxa de remoção (da ordem de $85 \%$ ), mas somente após oito horas de processo. Este estrogênio sintético não sofreu biodegradação, porém também foi removido pela sorção no lodo sedimentado. No geral, praticamente todas as variantes ensaiadas apresentaram maior eficiência na degradação/sorção dos estrogênios quando se adotou maiores TRS.

Shi et al. (2013) também relataram a degradação do estradiol à estrona em um reator independente da fase do processo (anóxica ou aeróbia). Porém, a transformação destes estrogênios na fase aeróbia foi mais intensificada. Pessoa et al. (2014) também encontraram maiores concentrações de estrona do que estradiol no efluente tratado por lodos ativados, devido à sua biodegradação aeróbia. O estudo de Shi et al. (2013) mostrou também que os estrogênios podem ser transferidos da fase líquida para o lodo, mostrando que os fenômenos de sorção podem ser os predominantes no processo de remoção desses compostos.

Kumar et al. (2011) obtiveram altas taxas de remoção dos estrogênios com valores acima de $80 \%$ em um processo por lodos ativados convencional adotando altos valores do TRS. Esses autores constataram que quanto menor a idade do lodo, menor é a taxa de remoção de estrogênios, o que corrobora com os resultados encontrados por Hashimoto e Murakami (2009). Entretanto, Petrie et al. (2014) obtiveram remoção de até 70\% de estrogênios com o TRS de três a dez dias. Isso mostra que ainda não existe um consenso quanto as variações dos parâmetros de controle dos processos de lodos ativados para uma melhor taxa de remoção e/ou degradação desses estrogênios.

No que diz respeito à eficiência de remoção de estrogênios em relação à operação em ambientes aeróbio, anóxico ou anaeróbio, estudos relataram que os sistemas aeróbios foram os mais eficazes. Ivanov et al. (2010) realizaram um estudo utilizando bactérias anaeróbias e facultativas com capacidade de redução de ferro para verificar a biodegradação dos estrogênios. Neste processo, tanto estrona quanto estriol foram reduzidos em $60 \%$ e $27 \%$, respectivamente. Com 15 dias de processo foi observada a remoção completa da estrona. Por outro lado, somente $9 \%$ do etinilestradiol foi removido, provavelmente pelo mecanismo de 
sorção. Este estudo indica que nos processos anaeróbios existem mecanismos de degradação diferentes do aeróbio, mas que a remoção pela sorção no lodo também pode ocorrer.

Com o propósito de avaliar o potencial de remoção e/ou degradação de estrogênios utilizando fontes alternativas de carbono orgânico, Racz et al. (2012) utilizaram dois reatores em bateladas sequenciais (RBS) em escala laboratorial utilizando esgoto sintético, onde em um foi adicionada peptona e, no outro, glicose como fonte de carbono. Os autores observaram uma maior eficiência de sorção pelo lodo no reator com peptona, o que foi explicado pela alta taxa de nitrificação. Este estudo sugere ainda que, sistemas aeróbios nitrificantes podem ter uma eficiência maior na remoção principalmente do estrogênio sintético etinilestradiol.

Além dos sistemas por lodos ativados e os anaeróbios, outros processos têm sido testados quanto a remoção de estrogênios como os sistemas de alagados (wetland) e lagoas (estabilização, maturação e suas diversas variantes). Pessoa et al. (2014) avaliaram a eficiência de remoção da estrona, estradiol, e etinilestradiol em quatro tipos de processos distintos em escala real: uma lagoa facultativa seguida por uma lagoa de maturação, uma lagoa facultativa, um sistema de lodos ativados e um reator UASB. As taxas de remoção desses estrogênios pelas lagoas foram abaixo de $70 \%$, enquanto que os processos de lodos ativados e reator UASB tiveram uma eficiência acima de $90 \%$.

Song et al. (2009) estudaram a remoção da estrona, estradiol e etinilestradiol em diferentes profundidades de um sistema de alagados (wetland) em escala piloto como pós-tratamento de lodos ativados. Os autores observaram uma maior eficiência (da ordem de 75\%), na remoção de estrogênios nas camadas mais superficiais do alagado, onde provavelmente prevalece o metabolismo aeróbio. Este estudo relatou que a biodegradação e a sorção foram os mecanismos predominantes nas zonas da rizosfera, onde prevalecem as raízes secundárias das plantas (raízes mais finas). Logo, micro-organismos presentes nas raízes das plantas podem estar atuando nos mecanismos de remoção e/ou degradação desses compostos.

Portanto, com base nos estudos consultados é possível observar que os processos aeróbios por lodos ativados parecem ser os mais promissores na remoção de estrogênios, sobretudo por serem os sistemas mais citados na literatura sobre este assunto. No entanto, para que seja consolidado este conhecimento é necessário associar alguns dos parâmetros operacionais adotados nesses sistemas como idade do lodo, TDH e taxa de aeração, por exemplo, com a eficiência de remoção e/ou degradação desses compostos. Além disso, faz-se pertinente analisar como a adoção de processos anóxicos e/ou anaeróbios podem influenciar nas condições metabólicas, e por consequência na remoção e/ou degradação destes compostos.

Um ponto ainda pouco estudado e que merece atenção é o fato de, ao se desencadear o processo de biodegradação dos estrogênios, haver a produção de metabólitos. Desta forma, é importante que mais estudos investiguem tais mecanismos de biodegradação ao longo dos sistemas e, sobretudo, identifiquem quais subprodutos podem ser formados e seus respectivos potenciais de desregulação endócrina.

\section{CONCLUSÃO}

Os estrogênios apresentam-se como importantes contaminantes emergentes que podem afetar a saúde ambiental e humana. Estes têm no lançamento de esgotos domésticos sua principal via de introdução no meio ambiente. Estudos realizados em diversos países relataram concentrações significativas desses compostos em esgoto bruto e tratado, bem como nas águas superficiais que recebem esgoto doméstico. Apesar de existirem poucos artigos sobre este assunto, os níveis desses contaminantes no Brasil são maiores do que os encontrados nos demais países. Os sistemas de tratamento de esgotos podem representar uma importante barreira neste processo de contaminação do meio hídrico. Entretanto, no Brasil, em decorrência dos baixos 
índices de coleta e tratamento de esgotos, ainda se observa que boa parte dos estrogênios excretados acaba tendo como destino final os corpos hídricos (SNIS, 2013).

Os processos aeróbios, sobretudo os por lodos ativados, mostraram melhor desempenho na biodegradação desses compostos. A sorção pelo lodo biológico parece ser o principal mecanismo na remoção de estrogênios; no entanto, ainda é necessário conhecer melhor os mecanismos de sua biodegradação. Há uma vasta gama de possibilidades tecnológicas que podem remover e/ou degradar esses compostos. Porém ainda são necessários mais estudos sobre a utilização destas tecnologias de tratamento no Brasil. Esta área de conhecimento merece maior atenção, uma vez que as ETEs podem atuar como importantes barreiras, tanto à introdução de estrogênios, quanto de outros micropoluentes emergentes.

\section{AGRADECIMENTOS}

Os autores agradecem ao estatístico Felipe Pinto pelo apoio na análise e apresentação gráfica dos dados, à Capes pela concessão de bolsa de doutorado, à Faperj (Processos E26/110.102/2014 e E-26/201.479/2014) e ao CNPq (Processo 45533/2014-7) pelo apoio a pesquisa.

\section{REFERÊNCIAS}

AQUINO, S. F.; BRANDT, E. M. F.; CHERNICHARO, C. A. L. Remoção de fármacos e desreguladores endócrinos em estações de tratamento de esgoto: revisão da literatura. Engenharia Sanitária e Ambiental, v.18, n. 3, p. 187-204, 2013. http://dx.doi.org/10.1590/S1413-41522013000300002

ATKINSON, S. K.; MARLATT, V. L.; KIMPE, L. E. et al. The occurrence of steroidal estrogens in south-eastern Ontario wastewater treatment plants. Science of the Total Environment, v. 430, p. 119-125, 2012.

http://dx.doi.org/10.1016/j.scitotenv.2012.04.069.

BARCELÓ, D.; PETROVIC, M. Emerging contaminants from industrial and municipal waste: occurrence, analysis and effects. Berlin: Springer; c2008. 206p.

BERGMAN, A.; HEINDEL, J. J.; JOBLING, S. et al. State of the science of endocrine disrupting chemicals. Genebra: UNEP; WHO, c2012. 180p.

BILA, D. M.; DEZOTTI, M. Desreguladores endócrinos no meio ambiente: efeitos e consequências. Química Nova, v. 30, n. 3, p. 651-666, 2007. http://dx.doi.org/10.1590/S0100-40422007000300027.

BRANDT, E. M. F; QUEIROZ, F. B.; AFONSO, R. J. C. F. et al. Behaviour of pharmaceuticals and endocrine disrupting chemicals in simplified sewage treatment systems. Journal of

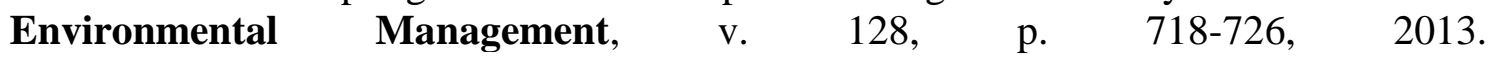
http://dx.doi.org/10.1016/j.jenvman.2013.06.003.

CANO, A.; ROURA, A. C.; CORTIT, L. I. Farmacología de los anticonceptivos hormonales orales. In: BUIL, C. (Ed.). Manual de anticoncepcíon hormonal oral. Zaragoza: Sociedad Española de Contracepción, 1997. p. 75-99.

CETESB - COMPANHIA DE TECNOLOGIA DE SANEAMENTO AMBIENTAL. Relatório de estabelecimento de valores orientadores para solos e águas subterrâneas no Estado de São Paulo. São Paulo, 2001. 
CHIMCHIRIAN, R. F.; SURI, R. P. S.; FU, H. Free synthetic and natural estrogen hormones in influent and effluent of three municipal wastewater treatment plants. Water $\begin{array}{llllllll}\text { Environment } & \text { Research, } & \text { v. 79, } & \text { n. 9, }\end{array}$ http://www.jstor.org/stable/25053673.

COMBALBERT, S.; HERNANDEZ-RAQUET, G. Occurrence, fate, and biodegradation of estrogens in sewage and manure. Applied Microbiology and Biotechnology, v. 86, n. 6, p. 1671-1692, 2010. http://dx.doi.org/10.1007/s00253-010-2547-x

CUNHA, D. L.; SILVA, S. M. C.; BILA, D. M. et al. Regulamentação do estrogênio sintético $17 \alpha$-etinilestradiol em matrizes aquáticas na Europa, Estados Unidos e Brasil. Cadernos de Saúde Pública, v. 32, n. 3, p. 1-13, 2016. http://dx.doi.org/10.1590/0102311 X00056715.

D'ASCENZO, G.; DI CORCIA, A.; GENTILI, A. et al. Fate of natural estrogen conjugates in municipal sewage transport and treatment facilities. Science of the Total Environment, v. 302, n. 1-3, p. 199-209, 2003. http://dx.doi.org/10.1016/S0048-9697(02)00342-X

DREWES, J. E.; HEMMING, J.; LADENBURGER, S. et al. An assessment of endocrine disrupting activity changes in water reclamation systems through the use of bioassays and chemical measurements. Water Environment Research, v. 77, n. 1, p. 12-23, 2005. http://dx.doi.org/10.2175/106143005X41573.

DUONG, C. N.; RA, J. S.; CHO J. et al. Estrogenic chemicals and estrogenicity in river waters of South Korea and seven Asian countries. Chemosphere, v. 78, p. 286-293, 2010. http://dx.doi.org/10.1016/j.chemosphere.2009.10.048.

FENT, K.; WESTON, A. A.; CAMINADA, D. Ecotoxicology of human pharmaceuticals. Aquatic toxicology, v. 76, n. 2, p. 122-159, 2006. http://dx.doi.org/10.1016/j.aquatox.2005.09.009

FROEHNER, S.; PICCIONI, W.; MACHADO, K. S.; AISSE, M. M. Removal capacity of caffeine, hormones, and bisphenol by aerobic and anaerobic sewage treatment. Water, Air \& Soil Pollution, v. 216, n. 1, p. 463-471, 2011. http://dx.doi.org/10.1007/s11270010-0545-3.

GARMSHAUSEN, J.; KLOAS, W.; HOFFMANN, F. 17 $\alpha$-ethinylestradiol can disrupt hemoglobin catabolism in amphibians. Comparative Biochemistry and Physiology Part C: Toxicology \& Pharmacology, v. 171, p. 34-40, 2015. http://dx.doi.org/10.1016/j.cbpc.2015.03.004.

GILBERT, N. Drug-pollution law all washed up. Nature, v. 491, n. 7425, p. 503-504, 2012. http://dx.doi.org/10.1038/491503a.

GIUSTI, A.; LAGADIC, L.; BARSI, A. et al. Investigating apical adverse effects of four endocrine active substances in the freshwater gastropod Lymnaea stagnalis. Science of $\begin{array}{llllll}\text { the Total Environment, } & \text { v. 493, p. 147-155, }\end{array}$ http://dx.doi.org/10.1016/j.scitotenv.2014.05.130.

GÓMEZ, M. J.; HERRERA, S.; SOLÉ, D. et al. Spatio-temporal evaluation of organic contaminants and their transformation products along a river basin affected by urban, agricultural and industrial pollution. Science of The Total Environment, v. 420, p.134145, 2012. http://dx.doi.org/10.1016/j.scitotenv.2012.01.029. 
HAMID, H; ESKICIOGLU, C. Fate of estrogenic hormones in wastewater and sludge treatment: A review of properties and analytical detection techniques in sludge matrix. $\begin{array}{lllllll}\text { Water Research, } & \text { v. 46, n. 18, p. 5813-33, }\end{array}$ http://dx.doi.org/10.1016/j.watres.2012.08.002.

HASHIMOTO, T.; MURAKAMI, T. Removal and degradation characteristics of natural and synthetic estrogens by activated sludge in batch experiments. Water Research, v. 43, n. 3, p. 573-582, 2009. http://dx.doi.org/10.1016/j.watres.2008.10.051.

IARC - INTERNATIONAL AGENCY FOR RESEARCH ON CANCER. IARC monographs on the evaluation of carcinogenic risks to humans. Lyon, 2007.

IVANOV, V.; LIM, J.; STABNIKOVA, O.; GIN, K. Biodegradation of estrogens by facultative anaerobic iron-reducing bacteria. Process Biochemistry, v. 45, n. 2, 284-287, 2010. http://dx.doi.org/10.1016/j.procbio.2009.09.017.

JIN, S.; YANG, F.; XU, Y.; DAI, H.; LIU, W. Risk assessment of xenoestrogens in a typical domestic sewage-holding lake in China. Chemosphere, v. 93, n. 6, p. 892-8, 2013. http://dx.doi.org/10.1016/j.chemosphere.2013.05.037.

KIM, S. D.; CHO, J.; KIM, In S. et al. Occurrence and removal of pharmaceuticals and endocrine disruptors in South Korean surface, drinking, and waste waters. Water research, v. 41, n. 5, p. 1013-1021, 2007. http://dx.doi.org/10.1016/j.watres.2006.06.034

KUMAR, V.; NAKADA, N.; YASOJIMA, M. et al. The arrival and discharge of conjugated estrogens from a range of different sewage treatment plants in the UK. Chemosphere, v. 82, n. 8, p. 1124-1128, 2011. http://dx.doi.org/10.1016/j.chemosphere.2010.11.040.

LIU, J.; LU, G.; XIE, Z. et al. Occurrence, bioaccumulation and risk assessment of lipophilic pharmaceutically active compounds in the downstream rivers of sewage treatment plants. Science of the Total Environment, v. 511, p. 54-62, 2015. http://dx.doi.org/10.1016/j.scitotenv.2014.12.033.

LUNA, T. O.; PLAUTZ, S. C.; SALICE, C. J. Chronic effects of 17 $\alpha$-ethinylestradiol, fluoxetine, and the mixture on individual and population-level end points in Daphnia magna. Archives of Environmental Contamination and Toxicology, v. 68, n. 4, p. 603-611, 2015. http://dx.doi.org/10.1007/s00244-014-0119-2.

MANICKUM, T.; JOHN, W. Occurrence, fate and environmental risk assessment of endocrine disrupting compounds at the wastewater treatment works in Pietermaritzburg (South Africa). Science of the Total Environment, v. 468-469, p. 584-597, 2014. http://dx.doi.org/10.1016/j.scitotenv.2013.08.041.

MARTIN, J.; CAMACHO-MUÑOZ, D.; SANTOS, J. L. et al. Occurrence of pharmaceutical compounds in wastewater and sludge from wastewater treatment plants: Removal and ecotoxicological impact of wastewater discharges and sludge disposal. Journal of Hazardous Materials, v. 239-240, p. 40-47, 2012. http://dx.doi.org/10.1016/j.jhazmat.2012.04.068.

MONTAGNER, C. C.; JARDIM, W. F. Spatial and seasonal variations of pharmaceuticals and endocrine disruptors in the Atibaia river, São Paulo State (Brazil). Journal of the Brazilian Chemical Society, v. 22, n. 8, p. 1452-1462, 2011. http://dx.doi.org/10.1590/S0103-50532011000800008. 
MOREIRA, D. S.; AQUINO, S. F.; AFONSO, R. J. C. F. et al. Occurrence of endocrine disrupting compounds in water sources of Belo Horizonte Metropolitan Area, Brazil.

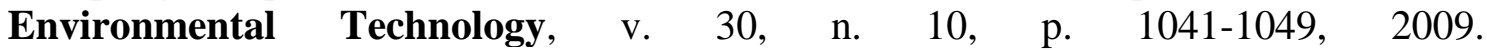
http://dx.doi.org/10.1080/09593330903052830.

MOREIRA, M. A.; AQUINO, S. F.; COUTRIM, M. X. et al. Determination of endocrinedisrupting compounds in waters from Rio das Velhas, Brazil, by liquid chromatography/high resolution mass spectrometry (ESILC-IT-TOF/MS). Environmental Technology, v. 32, n. 11-12, p. 1409-1417, 2011. http://dx.doi.org/10.1080/09593330.2010.537829.

MORTEANI, G.; MÖLLER, P.; FUGANTI, A.; PACES, T. Input and fate of anthropogenic estrogens and gadolinium in surface water and sewage plants in the hydrological basin of Prague (Czech Republic). Environmental Geochemistry and Health, v. 28, n. 3, p. 25764, 2006. http://dx.doi.org/10.1007/s10653-006-9040-6.

PAL, A.; GIN, K. Y. H.; LIN, A. Y. C.; REINHARD, M. Impacts of emerging organic contaminants on freshwater resources: review of recent occurrences, sources, fate and effects. Science of The Total Environment, v. 408, n. 24, p. 6062-6069, 2010. http://dx.doi.org/10.1016/j.scitotenv.2010.09.026.

PESSOA, G. P.; SOUZA, N. C.; VIDAL, C. B. et al. Occurrence and removal of estrogens in Brazilian wastewater treatment plants. Science of the Total Environment, v. 490, 288295, 2014. http://dx.doi.org/10.1016/j.scitotenv.2014.05.008.

PETRIE, B.; McADAM, E.; HASSARD, F. et al. Diagnostic investigation of steroid estrogen removal by activated sludge at varying solids retention time. Chemosphere, v. 113, p. 101-108, 2014. http://dx.doi.org/10.1016/j.chemosphere.2014.04.051.

QUEIROZ, F. B.; BRANDT, E. M. F.; AQUINO, S. F. et al. Occurrence of pharmaceuticals and endocrine disruptors in raw sewage and their behavior in UASB reactors operated at different hydraulic retention times. Water Science \& Technology, v. 6612, p. 25622569, 2012. http://dx.doi.org/10.2166/wst.2012.482

RACZ, L.; MULLER, J.; GOEL, R. Fate of selected estrogens in two laboratory scale sequencing batch reactors fed with different organic carbon sources under varying solids retention times. Bioresource Technology, v. 110, p. 35-42, 2012. http://dx.doi.org/10.1016/j.biortech.2012.01.059.

SHI, J.; CHEN, Q.; LIU, X. et al. Sludge/water partition and biochemical transformation of estrone and $17 \beta$-estradiol in a pilot-scale step-feed anoxic/oxic wastewater treatment system. Biochemical Engineering Journal, v. 74, p. 107-114, 2013. http://dx.doi.org/10.1016/j.bej.2013.03.001.

SILVA, P.; ROCHA, M. J.; CRUZEIRO, C. et al. Testing the effects of ethinylestradiol and of an environmentally relevant mixture of xenoestrogens as found in the Douro River (Portugal) on the maturation of fish gonads--a stereological study using the zebrafish (Danio rerio) as model. Aquatic Toxicology, v. 124-125, p. 1-10, 2012. http://dx.doi.org/10.1016/j.aquatox.2012.07.002.

SNIS - SISTEMA NACIONAL DE INFORMAÇÃO EM SANEAMENTO (Brasil). Série Histórica. 2013. Disponível em: http://app.cidades.gov.br/serieHistorica/manuais/ae/ diagnosticos/DiagAE_2013.pdf. Acesso em: 2016. 
SODRÉ, F. F. M.; MONTAGNER, C. C.; LOCATELLI, M. A. F.; JARDIM, W. F. Ocorrência de interferentes endócrinos e produtos farmacêuticos em águas superficiais da Região de Campinas (SP, Brasil). Journal of the Brazilian Society of Ecotoxicology, v. 2, n. 2, p. 187-196, 2007.

SONG, H.; NAKANO, K.; TANIGUCHI, T. et al. Estrogen removal from treated municipal effluent in small-scale constructed wetland with different depth. Bioresource Technology, v. 100, n. 12, p. 2945-2951, 2009. http://dx.doi.org/10.1016/j.biortech.2009.

TERNES, T. A.; STUMPF, M.; MUELLER, J. et al. Behavior and occurrence of estrogens in municipal sewage treatment plants - 1: Investigations in German, Canada and Brazil. Science of the Total Environment, v. 225, n. 1-2, p. 81-90, 1999. http://dx.doi.org/10.1016/S0048-9697(98)00334-9.

WILLIAMS, R. J.; CHURCHLEY, J. H. et al. Comparing predicted against measured steroid estrogen concentrations and the associated risk in two United Kingdom river catchments. Environmental Toxicology and Chemistry, v. 31, n. 4, p. 892-898, 2012. http://dx.doi.org/10.1002/etc.1756.

WANG, G.; MA, P.; ZHANG, Q. et al. Endocrine disrupting chemicals in New Orleans surface waters and Mississippi Sound sediments. Journal of Environmental Monitoring, v. 14, n. 5, p. 1353-1364, 2012. http://dx.doi.org/10.1039/c2em30095h.

XU, N.; XU, Y.; XU, S. et al. Removal of estrogens in municipal wastewater treatment plants: A Chinese perspective. Environmental Pollution, v. 165, p. 215-224, 2012. http://dx.doi.org/10.1016/j.envpol.2011.12.025

YE, X.; GUO, X.; CUI, X. et al. Occurrence and removal of endocrine-disrupting chemicals in wastewater treatment plants in the Three Gorges Reservoir area, Chongqing, China. Journal of Environmental Monitoring, v. 14, n. 8, p. 2204-11, 2012. http://dx.doi.org/10.1039/c2em30258f.

ZHANG, Z.; FENG, Y.; GAO, P. et al. Occurrence and removal efficiencies of eight EDCs and estrogenicity in a STP. Journal of Environmental Monitoring, v. 13, n. 5 p. 1366-1373, 2011. http://dx.doi.org/10.1039/c0em00597e.

ZORITA, S.; MÅRTENSSON, L.; MATHIASSON, L. Occurrence and removal of pharmaceuticals in a municipal sewage treatment system in the south of Sweden. Science of the Total Environment, v. 407, n. 8, p. 2760-2770, 2009. http://dx.doi.org/10.1016/j.scitotenv.2008. 


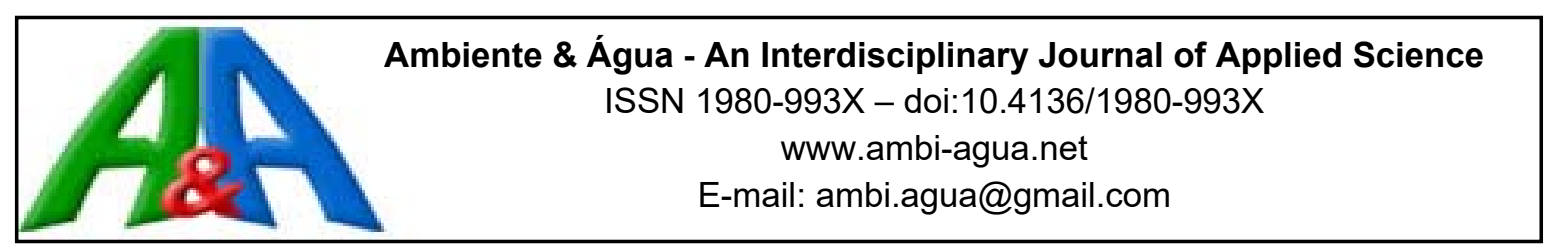

\title{
Atributos físicos e estoque de carbono do solo em áreas de Terra Preta Arqueológica da Amazônia
}

\author{
doi:10.4136/ambi-agua.1890 \\ Received: 11 Mar. 2016; Accepted: 31 Jan. 2017 \\ José Maurício da Cunha ${ }^{{ }^{*}}$; Denilton Carlos Gaio ${ }^{2}$;
Milton César Costa Campos ${ }^{1}$; Marcelo Dayron Rodrigues Soares $^{1}$;
Douglas Marcelo Pinheiro da Silva ${ }^{1}$; Alan Ferreira Leite de Lima ${ }^{1}$ \\ ${ }^{1}$ Universidade Federal do Amazonas (UFAM), Humaitá, AM, Brasil \\ Instituto de Educação, Agricultura e Ambiente (IEAA) \\ ${ }^{2}$ Universidade Federal de Mato Grosso (UFMT), Cuiabá, MT, Brasil \\ Programa de Pós-Graduação em Física Ambiental \\ *Autor correspondente: e-mail: maujmc@gmail.com, \\ dcgaio@ufmt.br, mcesarsolos@gmail.com, marcelo.dayron@gmail.com, \\ dougllas.marcelo@gmail.com, ala_leite@hotmail.com
}

\section{RESUMO}

Para se avaliar a qualidade do solo e a sustentabilidade em ambientes de Terra Preta Arqueológica da Amazônia, é importante a caracterização dos impactos de uso de solo dos diferentes tipos de cobertura. Sendo assim, o objetivo deste trabalho foi avaliar os atributos físicos e o carbono orgânico em áreas de Terra Preta Arqueológica, no município de Novo Aripuanã, AM, sob os cultivos feijão guandu (Cajanus cajan) e pastagem, em comparação com a cobertura de floresta, com base em técnicas de estatística tradicional univariada e multivariada e de geoestatística. Para isso foram delimitadas malhas com espaçamentos regulares e 88 pontos amostrais georreferenciados por malha. Foram coletadas amostras em blocos de solo com estrutura preservada e anéis volumétricos, para a determinação das propriedades físicas e do carbono orgânico do solo. O feijão guandu e pastagem diferem estatisticamente da floresta, que apresentaram características de estabilidade dos agregados acima da média, mas características texturais e carbono orgânico abaixo da média. O modelo exponencial, pelo semivariograma escalonado, mostrou que atributos físicos do solo apresentaram predominância, com grau de dependência de forte a moderado, sendo a área sob feijão guandu com maior variabilidade dos atributos do solo.

Palavras-chave: Aripuanã, química e física do solo, TPA sob floresta, usos do solo.

\section{Physical attributes and carbon stock of soils of Archaeological Dark Earth of the Amazonia}

\begin{abstract}
In order to evaluate soil quality and sustainability in Amazon Archaeological Dark Earth environments, it is important to characterize the impacts of the use of different cover types. The objective of this work was therefore to evaluate the physical attributes and organic carbon content in areas of "Terra Preta Arqueológica", in the municipality of Novo Aripuanã, AM, under the cultivation of pigeon pea (Cajanus cajan) and pasture, in comparison with forest
\end{abstract}


cover, based on traditional univariate and multivariate statistical techniques and geostatistics. In order to accomplish this, grids with regular spacing and samples with 88 georeferenced points per grid were delimited. Samples of soil blocks with preserved structure and volumetric rings were collected to determine physical properties and organic carbon content. The pigeon pea and pasture soils differ statistically from that of the forest and presented above-average aggregate stability, but texture and organic carbon characteristics were below average. The exponential model showed that the physical attributes of the soil predominated, with a strong to moderate degree of dependence, with the area under pigeon pea presenting the greatest variability of soil attributes.

Keywords: Aripuanã, land use, TPA under forest.

\section{INTRODUÇÃO}

As Terras Pretas Arqueológicas (TPA) são solos cuja característica marcante é a presença de coloração escura e artefatos cerâmicos e/ou líticos indígenas incorporados à matriz dos horizontes superficiais do solo (Kämpf e Kern, 2005). Elas apresentam uma elevada fertilidade natural incorporada à matriz do horizonte, sendo encontrada na região Amazônica em forma de manchas em meio a uma vasta extensão de terra que, na grande maioria, extremamente pobre em nutrientes.

Muitas dessas áreas são substituídas por atividades voltadas para fins industriais ou produção de alimentos. No entanto, solos preservados em seu estado natural, sob vegetação nativa, apresentam características físicas adequadas ao desenvolvimento das plantas (Calonego et al., 2012). Os usos de práticas agrícolas inadequada e de forma intensiva promovem alterações em suas propriedades originais (Rozane et al., 2010; Viana et al., 2011; Rossetti e Centurion, 2015), que por sua vez, a gestão dos solos de forma sustentável, com práticas de conservações apropriadas, é necessária a fim de que se possa evitar sua maior degradação (White et al., 2012).

Normalmente solos em ambientes naturais, por não sofrer interferência humana, apresentam menores densidade do solo e maiores volumes total de poros (Calonego et al., 2012), sendo que a densidade do solo apresenta correlação positiva com a microporosidade e negativa com a macroporosidade e porosidade total (Cunha et al., 2011), fatores que influenciam no processo de compactação do solo (Silveira et al., 2010).

Outros atributos usados para avaliar a qualidade física do solo são a resistência do solo a penetração (RP) e a umidade do solo, que por sua vez estão relacionados com crescimento e desenvolvimento radicular das plantas (Silveira et al., 2010). Em solos de ambientes naturais, foi observado menor RP em relação ao solo cultivado, ou seja, a densidade do solo é maior sob as plantas de cobertura, que reflete na maior resistência do solo à penetração, que por sua vez, tem apresentado correlação negativa com o teor de carbono orgânica no solo (Cunha et al., 2011; Silva et al., 2012).

A matéria orgânica tem influência direta e indiretamente aos atributos do solo (Viana et al., 2011) e o seu estudo em agro-ecossistema brasileiro é base para que se alcance a sustentabilidade agrícola (Cunha et al., 2011). É sabido também que práticas de uso e manejo dos solos, e suas variações, interferem no equilíbrio natural dos ecossistemas, alterando os componentes orgânicos tanto em quantidade como em qualidade (Melo e Schaefer, 2009). Por outro lado, o teor de carbono orgânico do solo sob floresta é maior do que em solos adjacentes que por sua vez favorece na estrutura do solo (Silva et al., 2008; Andrade et al., 2009).

$\mathrm{O}$ uso de práticas agrícolas tem efeito direto sobre os atributos físicos do solo, além de expressivas interferências no teor de carbono orgânico do solo (Matos et al., 2008; Rozane et 
al., 2010; Iori et al., 2012). Nesse sentido, a variabilidade espacial dos atributos físicos e químicos do solo, em ambientes naturais e transformados, torna-se importante para o entendimento dos processos de evolução a formação do solo e variações desses atributos para diferentes sistemas de uso e manejo do solo (Oliveira et al., 2013; 2015a; Aquino et al., 2015).

Há a necessidade de estudos relacionados aos atributos do solo de TPAs quanto a suas modificações em determinados usos e manejos dos solos, principalmente quando se compara com ambientes sob floresta. Pesquisa tem avaliado os atributos do solo sobre diferentes ambientes em ralação ao uso do solo sobre TPA (Oliveira et al., 2015b), identificando comportamento muito característico dos atributos do solo em ambientes de TPA. No entanto, não há estudos relacionados aos atributos do solo sobre o uso de TPA em comparação aos ambientes de floresta preservados em áreas de TPA.

Portanto, o objetivo deste trabalho foi avaliar os atributos físicos e o estoque de carbono em áreas de Terra Preta Arqueológica sob os cultivos feijão Guandu (FJ) (Cajanus cajan) e pastagem (PT), em comparação com a área de floresta (FN), utilizando técnicas de estatística tradicional univariada e multivariada e da geoestatística.

\section{MATERIAL E MÉTODOS}

O estudo localizou-se no município de Novo Aripuanã (Figura 1), Amazonas, Brasil, entre agosto de 2014 a julho de 2016, situado ao longo da rodovia Transamazônica (BR-230). O clima da região é Clima Tropical Chuvoso, apresentando um período seco de pequena duração. A pluviosidade média varia entre 2,250 e $2,750 \mathrm{~mm}$ ao ano, com período chuvoso entre outubro a junho. As temperaturas médias anuais variam entre $25^{\circ} \mathrm{C}$ e $27^{\circ} \mathrm{C}$ e a umidade relativa fica entre 85 e $90 \%$ (Brasil, 1978).
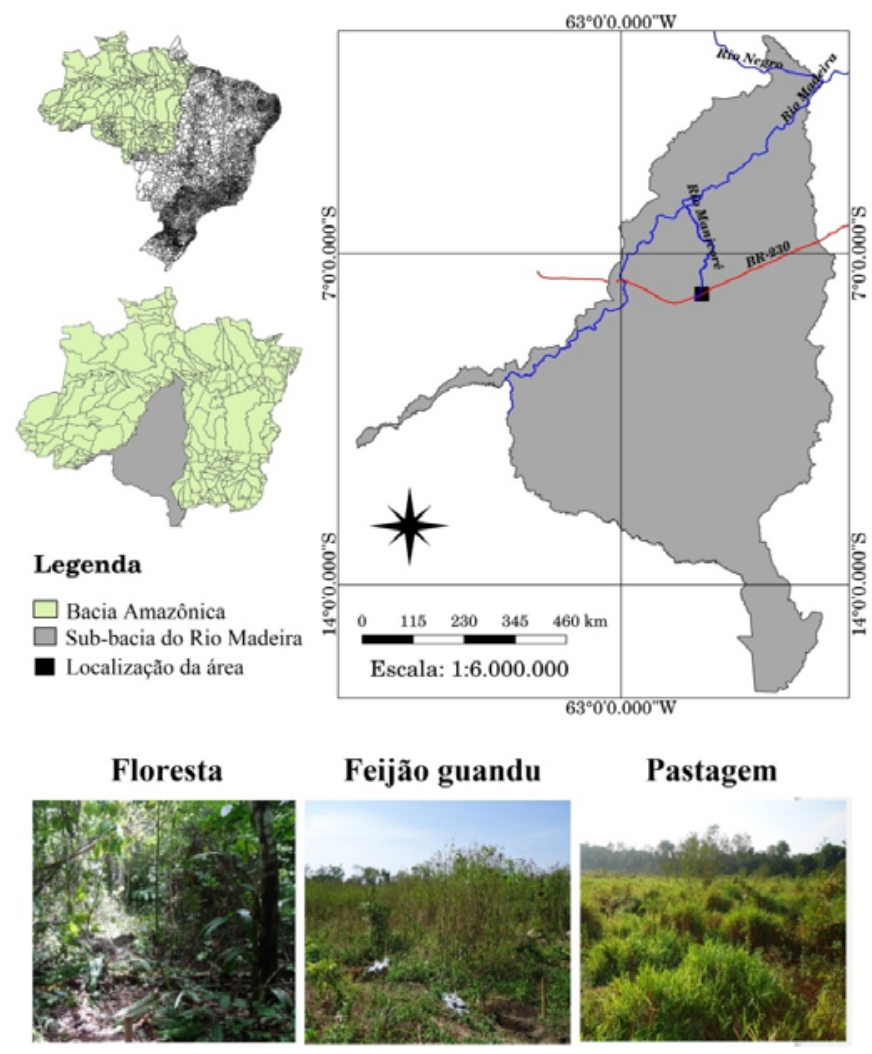

Figura 1. Localização da área de estudo.

A área de TPA sob floresta natural (FN) vem sendo preservado a mais de vinte e cinco anos, com início de recuperação florestal natural com árvores de porte mediano, chegando a 
apresentar de até quinze metros de altura. Já os usos de TPAs sob cultivo de feijão Guandu (Cajanus cajan) (FJ) e pastagem Brachiaria brizanta (PT), abrigou nos últimos vinte e cinco anos os cultivos milho, feijão e melancia. Nos últimos oito meses, foi inserido o pasto para atividades de pecuária, preservando uma área do feijão Guandu.

Foram delimitadas três malhas amostrais com 88 pontos de coleta de material por malha, sendo 90 pontos de coleta da área do feijão Guandu. As dimensões das malhas foram de $2.500 \mathrm{~m}^{2}$ para a FN, $1.700 \mathrm{~m}^{2}$ para o FJ e $4.800 \mathrm{~m}^{2}$ para a PT, com espaçamentos regulares entre os pontos de coleta do solo de $6 \times 6 \mathrm{~m}$, de $4 \times 5 \mathrm{~m}$ e de $8 \times 8 \mathrm{~m}$, respectivamente. A escolha das dimensões das malhas foi definida no campo, conforme avaliada as dimensões da área de TPA e a respectiva cobertura representativa do solo. Foram coletados blocos de solos com estrutura preservada e anéis volumétricos nas camadas $0,0-0,05 \mathrm{~m}, 0,05-0,10 \mathrm{~m}$ e 0,10-0,20 m, para a determinação das propriedades físicas estruturais, granulométricas e mecânicas do solo, assim como a matéria orgânica. Esses pontos foram georreferenciados com um equipamento de GPS para o mapeamento das áreas, e posteriormente utilizado nas análises geoestatística.

As amostras com estrutura preservada em forma de torrão de solo foram secadas à sombra e posteriormente destorroadas, de forma manual, e passadas em peneira de 9,51 $\mathrm{mm}$ de diâmetro e retidas na peneira de $4,76 \mathrm{~mm}$, para as análises relativas à estabilidade de agregados. As amostras retidas na peneira de $2,00 \mathrm{~mm}$ foram utilizadas nas análises granulométricas e de matéria orgânica, após passadas em peneira de $250 \mu \mathrm{m}$.

O método empregado para a separação e estabilidade dos agregados foi determinado segundo Kemper e Chepil (1965), com modificações nas seguintes classes de diâmetro: 4,76-2,0 mm; 2,0-1,0 mm; 1,0-0,50 mm; 0,50-0,25 mm;0,25-0,125 mm;0,125-0,063 mm. Os agregados foram colocados em contato com a água sobre a peneira de 2,00 $\mathrm{mm}$ e submetidos à agitação vertical em aparelho Yoder (SOLOTEST, Bela Vista, São Paulo, Brasil) por 15 min. Os materiais retidos em cada classe de peneira foram colocados em estufa a $105^{\circ} \mathrm{C}$, e em seguida mensurada as respectivas massas em uma balança digital.

Os resultados foram expressos em termos da porcentagem dos agregados retidos em cada uma das classes das peneiras e a estabilidade dos agregados avaliados pelo diâmetro médio ponderado (DMP) e o diâmetro médio geométrico (DMG), conforme Equações 1 e 2.

$$
\begin{aligned}
& D M P=\frac{\sum_{i=1}^{N} n_{i} D_{i}}{\sum n_{i}} \\
& D M G=10^{\frac{\sum_{i=1}^{N} n_{i} \log D_{i}}{\sum n_{i}}}
\end{aligned}
$$

em que:

$\mathrm{n}_{\mathrm{i}}=\%$ dos agregados retidos em uma determinada peneira;

$\mathrm{D}_{\mathrm{i}}=$ diâmetro médio de uma determinada peneira;

$\mathrm{N}=$ número de classes de peneiras.

A análise granulométrica foi realizada pelo método da pipeta, utilizando uma solução normal de $\mathrm{NaOH}$ como dispersante químico e agitação mecânica em aparato de alta rotação por 15 min, seguindo metodologia proposta pela Embrapa (2011). A fração argila foi separada por sedimentação, a areia por tamisação e o silte foi calculado por diferença.

Para as determinações da densidade do solo (Ds), macroporosidade (MaP) e microporosidade (MiP), volume total de poros (VTP) e umidade gravimétrica (Ug), as amostras de anéis volumétricos foram saturadas por meio da elevação gradual de uma lâmina de água numa bandeja plástica. Após a saturação, as amostras foram pesadas e levadas à mesa de tensão para determinação da MiP, sendo submetidas a uma tensão de 0,006 MPa (Embrapa, 2011).

Após atingirem o equilíbrio em um potencial matricial de $0,006 \mathrm{MPa}$, as amostras foram 
novamente pesadas e, em seguida, foram feitas as medidas da resistência do solo à penetração (RP), utilizando-se um penetrógrafo eletrônico modelo MA-933, marca Marconi. Posteriormente, as amostras foram levadas à estufa a $105^{\circ} \mathrm{C}$ para a determinação da Ug, Ds e VTP, pelo método do anel volumétrico, e a MaP foi determinada pela diferença entre VTP e MiP (Embrapa, 2011).

O carbono orgânico $(\mathrm{CO})$ foi determinado pelo método de Walkley-Black, modificado por Yeomans e Bremner (1988). A matéria orgânica é determinado pelo produto do CO com 1,724 (Embrapa, 2011). Já o estoque de carbono (EC) é definido pela Equação 3.

$$
E C=D s \times h \times C O
$$

em que:

EC: é estoque de carbono $\left(\mathrm{Mg} \mathrm{ha}^{-1}\right)$;

Ds: é densidade do solo $\left(\mathrm{Mg} \mathrm{m}^{-3}\right)$;

h: é a espessura da camada de solo amostrada $(\mathrm{cm})$; e

CO: é teor de C (\%).

Após a determinação dos atributos físicos e da matéria orgânica do solo, foram feitas análise estatística univariada e multivariada. A análise de variância univariada (ANOVA) foi utilizada para comparar médias dos atributos individualmente utilizando do teste de Tukey, tendo como referência a área de TPA sob floresta. Em seguida foi utilizada a análise de variância multivariada (MANOVA), através da análise fatorial e de agrupamento, a fim de encontrar significância estatística dos conjuntos dos atributos do solo que mais discriminam os ambientes, com referência ao ambiente sob floresta, obtendo como resposta atributos que sofrem maior influência sobre o uso do solo.

A adequação da análise fatorial foi feita pela medida de Kaiser-Meyer-Olkin (KMO), que avalia as correlações simples e parciais das variáveis, e pelo teste de esfericidade de Barlett, ao qual se pretende rejeitar a igualdade entre a matriz correlação com a identidade. A extração dos fatores foi feita pelas componentes principais, incorporando as variáveis que apresentaram comunalidades igual ou superior a cinco. A escolha do número de fatores a ser utilizados foi feito pelo critério de Kaiser (fatores que apresentam autovalores superior a 1). A fim de simplificar a análise fatorial, foi feita a rotação ortogonal (Varimax) dos fatores e representada em um plano fatorial das duas componentes.

A análise geoestatística foi feita com base no semivariograma experimental, estimado pela Equação 4.

$$
\hat{\gamma}(h)=\frac{1}{2 n(h)} \sum_{i=1}^{n(h)}\left[Z\left(x_{i}\right)-Z\left(x_{i}+h\right)\right]^{2}
$$

em que:

$\hat{y}(h)$ : é o valor da semivariância para uma distância h;

$\mathrm{n}(\mathrm{h})$ : é o número de pares envolvidos no cálculo da semivariância;

$\mathrm{Z}\left(\mathrm{x}_{\mathrm{i}}\right)$ : é o valor do atributo $\mathrm{Z}$ na posição $\mathrm{x}_{\mathrm{i}}$; e

$\mathrm{Z}\left(\mathrm{x}_{\mathrm{i}}+\mathrm{h}\right)$ : é o valor do atributo $\mathrm{Z}$ separado por uma distância $\mathrm{h}$ da posição $\mathrm{X}_{\mathrm{i}}$.

Para a análise do grau de dependência espacial das variáveis em estudo, utilizou-se a classificação de Cambardella et al. (1994), em que as propriedades do solo são consideradas com dependência espacial forte se a razão $\left[\frac{C_{0}}{C_{0}+C}\right]$ for $\leq 25$. Se essa razão estiver entre $26 \%$ a $75 \%$, a dependência espacial é considerada moderada, enquanto que se a propriedade do solo for maior que $75 \%$ a aproximadamente $95 \%$, classificam-se como dependência espacial fraca. 
Com base nos parâmetros dos semivariogramas experimentais dos atributos do solo, foram utilizados os semivariogramas escalonados com o objetivo de reduzi-los à mesma escala, facilitando a comparação entre resultados de diferentes variáveis (Ceddia et al., 2009). A escolha do modelo de semivariograma escalonado foi utilizada pelo menor resíduo. Os modelos utilizados nos ajustes do semivariograma escalonado foram o esférico (Equação 5) e o exponencial (Equação 6).

$$
\begin{aligned}
& \hat{y}(h)=C_{0}+C_{1}\left[\frac{3}{2}\left(\frac{h}{a}\right)-\frac{1}{2}\left(\frac{h}{a}\right)^{3}\right], \text { se } 0<h<a \\
& \hat{y}(h)=C_{0}+C_{1}=\left[1-\exp \left(-3 \frac{h}{a}\right)\right], 0<h<d
\end{aligned}
$$

em que:

$\mathrm{C}_{0}$ : é o efeito pepita;

$\mathrm{C}_{0}+\mathrm{C}_{1}$ : é o patamar;

$\left[\left(\mathrm{C}_{0} /\left(\mathrm{C}_{0}+\mathrm{C}_{1}\right)\right) \times 100\right]$ : é o grau de dependência espacial (GDE);

h: é a distância de separação entre duas observações; e

a: é o alcance do semivariograma. O ajuste do semivariograma foi feito com base no melhor coeficiente de determinação $\left(\mathrm{R}^{2}\right)$ e menor soma dos quadrados dos resíduos (SQRes).

A análise estatística ANOVA e MANOVA foi feita no programa computacional StatSoft, (2004), enquanto que a análise geoestatísitca foi utilizado o programa GS+ 7.0 (Robertson, 1998).

\section{RESULTADOS E DISCUSSÃO}

A estatística descritiva para os atributos em comparação aos usos do solo em áreas Terra Preta Arqueológica (TPA) é apresentada nas Tabelas 1, 2 e 3, para as respectivas camadas 0,00-0,05 m, 0,05-0,10 m e 0,10-0,20 m.

Os coeficientes de assimetria apresentaram valores próximos à zero, com exceção das classes de agregados $<1,00 \mathrm{~mm}$ para o uso de TPA sob pastagem, nas camadas $0,00-0,05 \mathrm{~m}$ e 0,05-0,10 m. Os resultados tendem a apresentar valores simétricos nos ambientes em estudo. Já o coeficiente de curtose, boa parte dos atributos apresentaram uma distribuição platicúrtica (distribuição mais achatada que a distribuição normal).

As medidas de média e mediana, devido aos dados apresentar uma distribuição simétrica, ambos mostraram valores muito próximo para todos os atributos e em suas respectivas camadas, o que justifica distribuições normais ou aproximadamente normais. Pelo teste de Kolmogorov-Smirnov, mostraram-se variações entre os ambientes e propriedades nas respectivas camadas. Mesmo assim, erros de análises em relação aos testes de hipóteses são mínimos, quando avaliados em relação a uma distribuição normal. Por outro lado, a normalidade dos dados não é um pressuposto necessário para a aplicação de técnica geoestatística, mas sim que a distribuição não apresente caudas muito alongadas, o que poderia comprometer a análise (Isaaks e Srivastava, 1989; Cressie, 1991).

Os atributos granulométricos apresentaram diferença significativa entre os ambientes estudados e com dominância na fração de areia, em contraste a resultados encontrados por Oliveira et al. (2015a), que evidenciou áreas de TPA sob cultivo com dominância na fração silte para a camada de 0,00-0,20 m. A área de TPA sob floresta evidenciou menores teores de areia para as três profundidades, e maiores teores de silte e argila, com exceção da fração argila na camada $0,10-0,20 \mathrm{~m}$, obtendo com isso menor valor. 
Tabela 1. Teste de média e estatística descritiva dos atributos do solo na profundidade de 0,00-0,05 m para as áreas com diferentes usos na região de Novo Aripuanã, Amazonas.

\begin{tabular}{|c|c|c|c|c|c|c|c|c|c|c|c|c|c|c|c|c|}
\hline \multirow{2}{*}{$\begin{array}{l}\text { Estatística } \\
\text { Descritiva }\end{array}$} & Ds & $\mathrm{MaP}$ & $\mathrm{MiP}$ & VTP & $\mathrm{Ug}$ & \multirow{2}{*}{$\begin{array}{c}\mathrm{RP} \\
\mathrm{MPa}\end{array}$} & \multirow{2}{*}{$\begin{array}{c}\mathrm{CO} \\
\mathrm{g} \mathrm{kg}^{-3}\end{array}$} & \multirow{2}{*}{$\begin{array}{c}\mathrm{EC} \\
\mathrm{tha}^{-1}\end{array}$} & DMG & DMP & \multirow{2}{*}{$>2,00$} & \multirow{2}{*}{$2,00-1,00$} & \multirow{2}{*}{$<1,00$} & \multirow{2}{*}{ Areia } & \multirow{2}{*}{$\begin{array}{l}\text { Silte } \\
\mathrm{g} \mathrm{kg}^{-1}\end{array}$} & \multirow{2}{*}{ Argila } \\
\hline & $\mathrm{mg} \mathrm{m}^{-3}$ & \multicolumn{3}{|c|}{$\%$} & & & & & \multicolumn{2}{|c|}{$\mathrm{mm}$} & & & & & & \\
\hline \multicolumn{17}{|c|}{ Floresta } \\
\hline Média & $1,27 \mathrm{~A}$ & $26,50 \mathrm{~A}$ & $30,90 \mathrm{~A}$ & $57,40 \mathrm{~A}$ & $24,40 \mathrm{~A}$ & $0,40 \mathrm{~B}$ & $22,96 \mathrm{~B}$ & $145,80 \mathrm{~B}$ & $2,74 \mathrm{~A}$ & $3,10 \mathrm{~A}$ & $91,19 \mathrm{~A}$ & $1,29 \mathrm{~B}$ & $8,05 \mathrm{~B}$ & $701,10 \mathrm{~B}$ & $203,40 \mathrm{~A}$ & $95,50 \mathrm{~A}$ \\
\hline Mediana & 1,27 & 26,10 & 30,70 & 57,50 & 24,30 & 0,37 & 22,17 & 141,70 & 2,76 & 3,10 & 91,17 & 1,17 & 7,43 & 697,30 & 199,50 & 94,60 \\
\hline DP & 0,08 & 3,59 & 2,19 & 3,30 & 2,40 & 0,13 & 4,50 & 25,80 & 0,26 & 0,12 & 3,96 & 0,73 & 3,88 & 27,40 & 27,60 & 16,02 \\
\hline CV (\%) & 6,30 & 13,55 & 7,09 & 5,75 & 9,84 & 32,50 & 19,60 & 17,82 & 9,49 & 3,87 & 4,34 & 56,59 & 48,20 & 3,91 & 13,57 & 16,77 \\
\hline Assimetria & 0,05 & 0,11 & 0,16 & $-0,03$ & 0,54 & 0,46 & 0,29 & 0,40 & $-0,58$ & $-0,57$ & $-0,56$ & 0,65 & 0,82 & $-0,15$ & $-0,05$ & $-0,03$ \\
\hline Curtose & 0,17 & $-0,16$ & 0,00 & 0,02 & 0,68 & $-0,02$ & $-0,53$ & $-0,02$ & $-0,22$ & $-0,29$ & $-0,29$ & $-0,21$ & 0,35 & 0,00 & $-0,38$ & $-0,50$ \\
\hline K-S & 0,20 & 0,20 & 0,20 & 0,20 & 0,20 & 0,03 & 0,20 & 0,20 & 0,06 & 0,20 & 0,20 & 0,20 & 0,20 & 0,20 & 0,20 & 0,20 \\
\hline \multicolumn{17}{|c|}{ Feijão Guandu } \\
\hline Média & $1,26 \mathrm{~A}$ & $26,40 \mathrm{~A}$ & $30,80 \mathrm{~A}$ & $57,20 \mathrm{~A}$ & $24,90 \mathrm{~A}$ & $0,49 \mathrm{~A}$ & $33,93 \mathrm{~A}$ & $213,76 \mathrm{~A}$ & $2,24 \mathrm{~B}$ & $2,80 \mathrm{~B}$ & 80,86 B & $2,58 \mathrm{~A}$ & $17,36 \mathrm{~A}$ & $732,50 \mathrm{~A}$ & $194,90 \mathrm{~A}$ & $72,60 \mathrm{~B}$ \\
\hline Mediana & 1,26 & 27,00 & 30,80 & 57,02 & 24,70 & 0,44 & 33,02 & 210,30 & 2,29 & 2,90 & 83,55 & 2,54 & 14,58 & 734,60 & 192,50 & 72,40 \\
\hline DP & 0,14 & 5,40 & 3,30 & 4,70 & 3,80 & 0,21 & 7,34 & 40,90 & 0,58 & 0,37 & 12,26 & 1,58 & 11,41 & 39,40 & 35,21 & 18,50 \\
\hline CV (\%) & 11,11 & 20,45 & 10,71 & 8,22 & 15,26 & 42,86 & 21,63 & 19,35 & 25,89 & 13,21 & 15,16 & 61,24 & 65,73 & 5,38 & 18,07 & 25,48 \\
\hline Assimetria & $-0,04$ & $-0,36$ & 0,19 & 0,12 & 0,40 & 0,78 & 0,04 & $-0,14$ & $-0,24$ & $-0,63$ & $-0,60$ & 0,66 & 0,59 & $-0,24$ & 0,22 & 0,16 \\
\hline Curtose & $-0,43$ & $-0,27$ & $-0,22$ & $-0,22$ & $-0,08$ & 0,30 & $-0,33$ & 0,00 & $-1,09$ & $-0,40$ & $-0,51$ & $-0,04$ & $-0,55$ & $-0,41$ & $-0,14$ & $-0,15$ \\
\hline K-S & 0,20 & 0,20 & 0,20 & 0,20 & 0,20 & 0,01 & 0,20 & 0,20 & 0,00 & 0,00 & 0,01 & 0,20 & 0,00 & 0,20 & 0,20 & 0,20 \\
\hline \multicolumn{17}{|c|}{ Pastagem } \\
\hline Média & $1,22 \mathrm{~A}$ & $27,70 \mathrm{~A}$ & $30,90 \mathrm{~A}$ & $58,60 \mathrm{~A}$ & $25,30 \mathrm{~A}$ & $0,49 \mathrm{~A}$ & $22,77 \mathrm{~B}$ & $138,90 \mathrm{~B}$ & $2,64 \mathrm{~A}$ & $3,10 \mathrm{~A}$ & $90,86 \mathrm{~A}$ & $0,93 \mathrm{C}$ & $8,47 \mathrm{~B}$ & $741,40 \mathrm{~A}$ & $180,50 \mathrm{~B}$ & $78,10 \mathrm{~B}$ \\
\hline Mediana & 1,22 & 28,30 & 31,10 & 58,30 & 25,30 & 0,44 & 22,51 & 133,30 & 2,65 & 3,11 & 91,85 & 0,80 & 7,34 & 745,60 & 180,40 & 78,00 \\
\hline DP & 0,10 & 6,60 & 3,60 & 4,50 & 2,60 & 0,22 & 4,25 & 28,40 & 0,26 & 0,13 & 4,33 & 0,56 & 4,28 & 29,40 & 34,30 & 18,97 \\
\hline CV (\%) & 8,20 & 23,83 & 11,65 & 7,68 & 10,28 & 44,90 & 18,66 & 20,42 & 9,85 & 4,19 & 4,77 & 60,22 & 50,53 & 3,97 & 19,00 & 24,29 \\
\hline Assimetria & 0,06 & $-0,22$ & 0,40 & 0,16 & 0,12 & 0,68 & 0,27 & 0,49 & $-0,48$ & $-1,00$ & $-1,00$ & 0,60 & 1,17 & $-0,42$ & $-0,25$ & 0,08 \\
\hline Curtose & $-0,16$ & $-0,36$ & $-0,40$ & $-0,72$ & $-0,51$ & 0,01 & $-0,35$ & $-0,02$ & $-0,18$ & 0,67 & 0,65 & $-0,51$ & 0,85 & 0,22 & $-0,06$ & $-0,25$ \\
\hline K-S & 0,20 & 0,20 & 0,01 & 0,20 & 0,20 & 0,06 & 0,20 & 0,20 & 0,20 & 0,01 & 0,01 & 0,04 & 0,01 & 0,08 & 0,20 & 0,20 \\
\hline
\end{tabular}

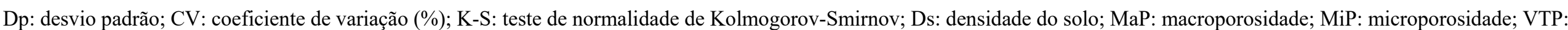

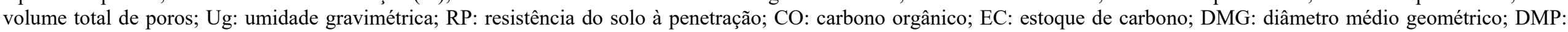
diâmetro médio ponderado. Médias seguidas pela mesma letra maiúscula na coluna não diferem entre si pelo teste de Tukey ( $\mathrm{p}<0,05$ ). 
Tabela 2. Teste de média e estatística descritiva dos atributos do solo na profundidade de 0,05-0,10 m para as áreas com diferentes usos na região de Novo Aripuanã, Amazonas.

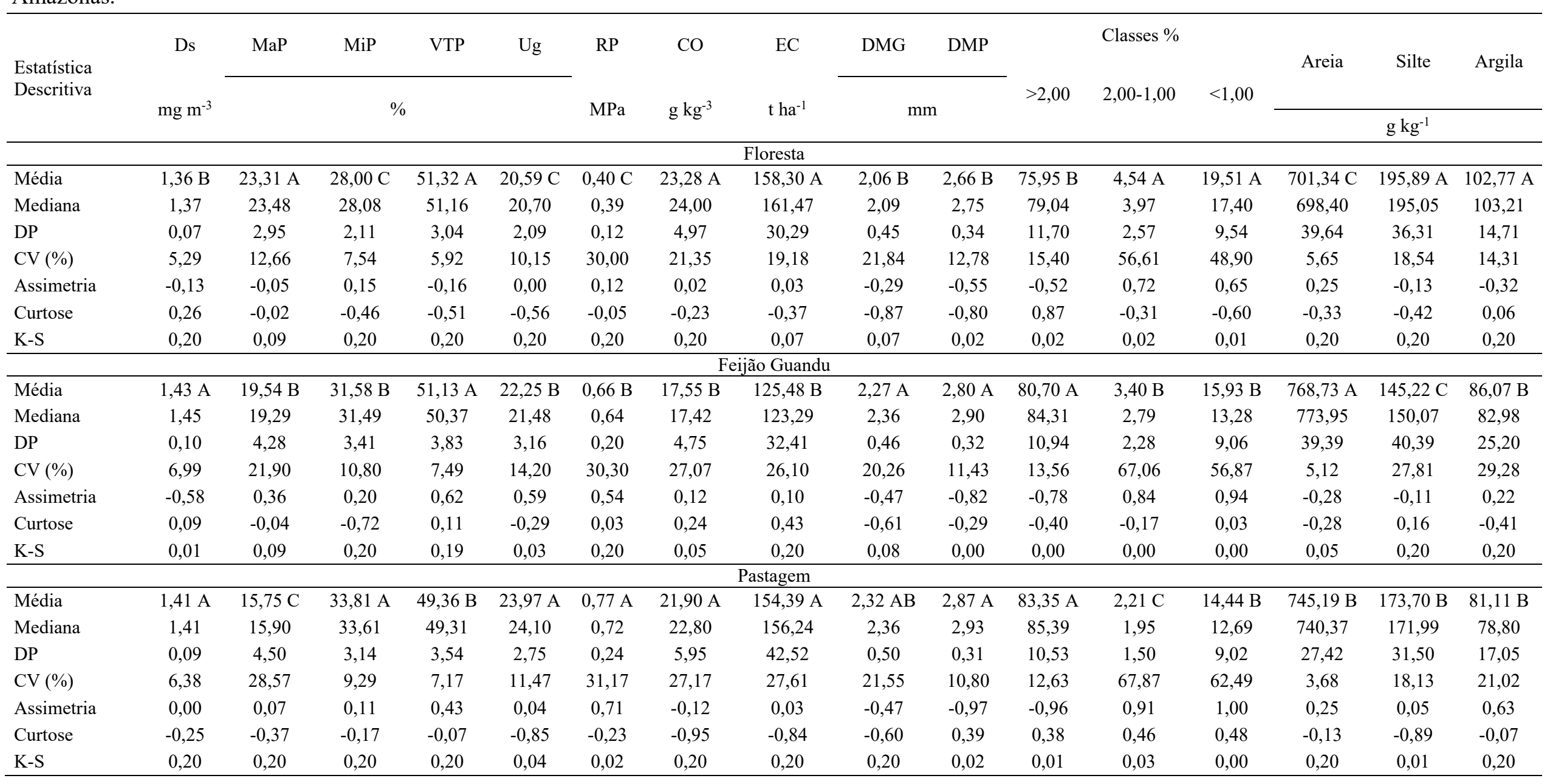

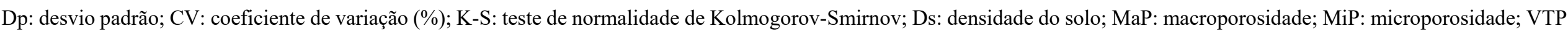

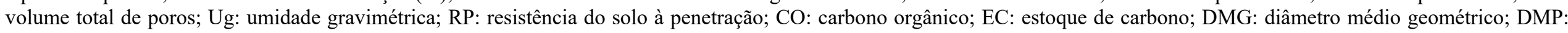
diâmetro médio ponderado. Médias seguidas pela mesma letra maiúscula na coluna não diferem entre si pelo teste de Tukey $(p<0,05)$.

Rev. Ambient. Água vol. 12 n. 2 Taubaté - Mar. / Apr. 2017 


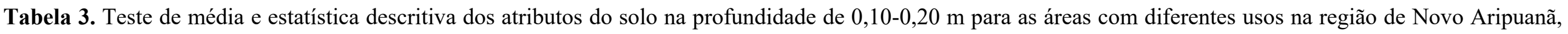
Amazonas.

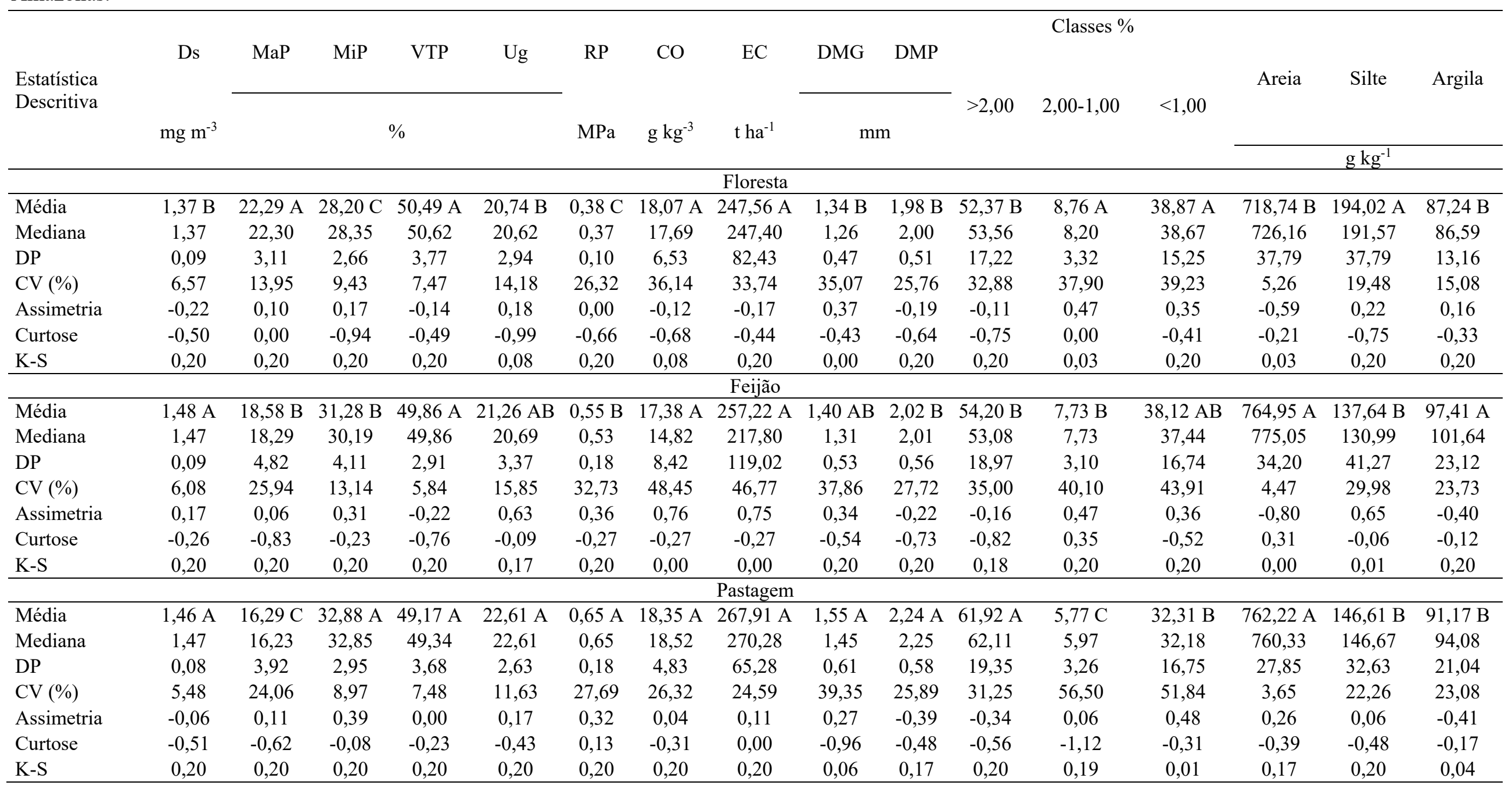

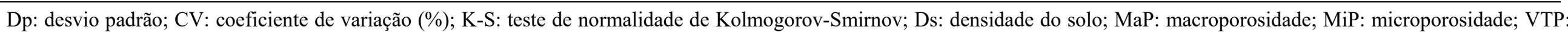

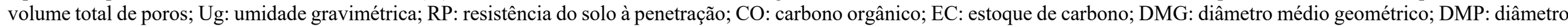
médio ponderado. Médias seguidas pela mesma letra maiúscula na coluna não diferem entre si pelo teste de Tukey $(\mathrm{p}<0,05)$. 
No estudo de gênese do solo, a fração silte passa a constituir como o indicador pela a ação de intemperismo, o que pudemos constatar solos de formação mais jovens que os propostos por Oliveira et al. (2015a).

Por outro lado, os maiores teores de areia sob as áreas de feijão guandu e pastagem se devem provavelmente a fortes chuvas que promovem grandes enxurradas, por essas apresentarem áreas mais abertas, com impacto das gotas de chuva sobre o solo em relação à área sob floresta. A presença de cobertura no solo atua na interceptação das gotas de chuva e evita o desprendimento das partículas do solo. Chuvas de grande intensidade causam maior desagregação do solo e, normalmente, originam escoamento superficial, sendo as partículas menores de solo são mais fáceis de serem transportadas (Volk e Cogo, 2009).

Interações significativas entre os sistemas de uso do solo foram verificadas para as propriedades físicas Ds, MaP, MiP, VTP e Ug, indicando que o uso do solo pouco afetou nas variações dessas propriedades (Tabelas 1,2 e 3 ), para a camada entre 0,00-0,05 m, mas houve mudanças de comportamento aos demais atributos e em camadas subsequentes, com exceção do VTP, CO e EC na camada 0,10-0,20 m. Por outro lado, na camada de 0,00-0,05 m, o solo mantido sob pastagem apresentou-se menos compactada, com menor valor de Ds, além de maiores valores do VTP em relação ao TPA sob floresta e feijão guandu. Mesmo assim, a Ds não diferiu muito em relação ao sistema de uso do solo dentro da mesma camada $(0,00-0,05$ $\mathrm{m})$, mas houve alterações para as camadas $0,05-0,10$ e $0,10-0,20 \mathrm{~m}$, com menor valor de Ds para o uso sob floresta, não havendo diferença entre os sistemas de uso sob feijão guandu e pastagem (Tabelas 1,2 e 3 ).

A ausência de práticas agressivas ao solo em florestas naturais afeta diretamente a estrutura do solo, com baixos valores de Ds (Calonego et al., 2012). Além disso, os mesmos autores afirmam que tanto os altos valores da MO quanto os elevados valores do VTP reduzem também os valores de Ds. Resultados semelhantes foram encontrados para solos não antropogênicos sob o horizonte A (Campos et al., 2012; Santos et al., 2013), mas que apresentaram valores muito baixo em solos antropogênicos (em TPA). Esse fato pode estar relacionado ao tipo de solo, com características físicas que diferem em ralação aos perfis do solo para determinados atributos. Em trabalho realizado por Santos et al. (2011), os autores verificaram valores semelhantes da Ds em TPA com perfil de Neossolo Litólico sob o uso de pastagem, mas com valores baixos em comparação com outros perfis.

Também não houve diferença significativas entre a MaP com os sistemas de uso na camada entre 0,00-0,05 m, que apresentou comportamento semelhante da MiP. No entanto, nas camadas de 0,05-0,10 e 0,10-0,20 m, observou-se maiores valores para a MaP sob o uso de floresta natural e de MiP sob o uso de pastagem, corroborando assim com os valores do VTP para os sistemas de uso do solo, que apresentaram maiores valores em floresta, apesar de haver diferença significativa apenas na camada entre 0,05-0,10 m, com menor valor no sistema de uso sob pastagem.

Esses resultados demonstram que os sistemas de uso do solo contribuem efetivamente para a melhora das condições físicas do solo, promovendo redução da Ds em relação à área de floresta. Resultados semelhantes também foram verificados por Cunha et al. (2011), em avaliar os atributos físicos de solo cultivado com feijão e milho orgânicos sob a influência das plantas de cobertura, que apresentaram, em solo sob mata nativa, menores valores de Ds e MiP e maiores valores de MaP e VTP. Para os autores, essas condições foram propícias devido ao solo não sofrer o trânsito de máquinas e equipamentos ou animais.

Em relação à resistência do solo à penetração (RP), houve diferença significativa sob os sistemas de uso do solo para as três camadas, cujos menores valores da RP estão associados ao uso sob floresta. Esses resultados corroboram também para o baixo valor da Ug e Ds sob a mesma área, apesar de não haver diferença significativa de Ug em relação aos sistemas de uso na camada 0,00-0,05 m. Em estudo sobre a relação entre a umidade do solo e a resistência à 
penetração de um Argissolo Amarelo distrocoeso na região do Recôncavo da Bahia, Silveira et al. (2010) constataram que solos com baixo conteúdo de água, apresentam partículas mais próximas e difíceis de serem separadas, com o aumento da RP. O solo sob floresta apresentou valores de RP menores que $0,50 \mathrm{MPa}$ nos $0,20 \mathrm{~m}$ de profundidade, diferindo dos solos manejados, que apresentaram solos menos compactados apenas nos primeiros $0,05 \mathrm{~m}$ de profundidade. Mesmo assim, esses valores ainda são muito baixos se considerarmos os valores limites definidos do Tavares Filho e Tessier (2009) para solos compactados (RP de 2,0 MPa). Em solos de TPA sob o uso de pastagem, verificou-se valores acima de 2,0 MPa para uma camada de 0,0-0,05 mm (Soares et al., 2015) que, segundo os autores, justifica-se pela compactação do solo devido ao pisoteio animal.

Tanto o CO quanto o EC (Tabelas 1, 2 e 3) houve alterações para os respectivos usos do solo, com maiores valores sob o uso de feijão Guandu na camada 0,00-0,05 m, passando a ter menor valor na camada $0,05-0,10 \mathrm{~m}$, não havendo diferença estatística entre os usos na camada 0,10-0,20 m. Os valores altos de CO sob feijão Guandu na camada 0,00-0,05 m devem estar associados à elevada cobertura vegetal, com mais de oito meses de plantio sem sofrer práticas intensivas no solo e trânsitos de animais.

Altas concentrações de cobertura vegetal verificadas in loco sob o uso do feijão Guandu pode ter favorecido aos maiores teores de CO. Esse resultado difere do proposto por Rossetti et al. (2015), ao determinar o efeito do sistema plantio direto sobre os estoque de carbono e atributos físicos do solo cultivados com soja e milho, e comparação com a mata nativa, identificaram maior concentração de MO sob mata nativa em relação aos demais tratamentos, ao qual os autores associam à deposição contínua de serrapilheira, além da ausência de ação antrópica.

Sob os respectivos usos do solo, o DMG e DMP diferiram estatisticamente, dando maiores valores sob o uso de floresta para a camada $0,00-0,05 \mathrm{~m}$, e sob o uso de pastagem para as camadas de 0,05-0,10 m e 0,10-0,20 m. No entanto, não foram confirmadas correlações significativas entre o DMG/DMP com os atributos CO e EC nas camadas de 0,00-0,05 m e 0,05-0,10 m, com exceção sob o uso de feijão Guandu na camada 0,05-0,10 m, que obteve correlações positivas para o $\mathrm{CO}(0,25$ e 0,27 , respectivamente).

Por outro lado, na camada 0,10-0,20 m, houve correlações significativas entre o DMG/DMP com o CO e EC, com valores de coeficiente de correlação definidos em: 0,39/0,39 e $0,38 / 0,38$, respectivamente, para o uso sob floresta; de $0,36 / 0,36$ e $0,35 / 0,35$, respectivamente, sob o uso sob pastagem; e de 0,30/0,30 e 0,27/0,27, respectivamente, sob o uso de feijão Guandu. Correlações positivas do DMG e DMP com o carbono orgânico foram verificadas por Rozane et al. (2010), indicando sua importância na agregação do solo. Esses resultados também corroboram com os Matos et al. (2008), quando avaliaram a estabilidade de agregados sobre o impacto da adubação orgânica e mineral sobre o solo. $O$ fato de haver perda de correlação dos atributos DMG e DMP com o CO e EC nas camadas mais superficiais, pode ser explicado por práticas como a aração, gradagem e o pisoteio do gado, que promovem a desestruturação dos agregados e a oxidação da matéria orgânica, além do revolvimento do solo para camadas mais profundas.

$\mathrm{Na}$ análise multivariada, foi possível observar os atributos do solo que sofreu maior alteração quando ao uso do solo, em comparação a área sob floresta. A adequação da análise fatorial mostrou-se significativo (KMO igual a 0,78 e p $<0,05$ para o teste de esfericidade de Barlett) para os atributos avaliados. Na análise de componentes principais (ACP), o número de fatores a ser extraído foi estabelecido de forma a explicar acima de $70 \%$ da variância total dos dados (Tabela 4 e Figura 2), que apresentaram autovalores da matriz de covariância superior a um (1) (Manly, 2008), com 4,71 na CP1 e 3,80 na CP2. Do percentual da variância explicada, observou-se que a CP1 é responsável por $42,79 \%$ da variância total, enquanto que a CP2 é responsável por $34,54 \%$. 
Tabela 4. Correlação entre cada componente principal e variáveis analisadas e análise fatorial dos atributos do solo com os fatores rotacionados (Varimax) (Fator 1 e 2 ) correspondentes aos ambientes estudados, na região de Novo Aripuanã, Amazonas.

\begin{tabular}{lccc}
\hline \multirow{2}{*}{ Atributos } & Variância & \multicolumn{2}{c}{ Fatores } \\
\cline { 3 - 4 } & Comum & PC1 & PC2 \\
\hline Ds & 0,79 & $-0,15$ & $-0,86^{*}$ \\
VTP & 0,75 & 0,09 & $0,80^{*}$ \\
Ug & 0,61 & 0,25 & $0,74^{*}$ \\
CO & 0,44 & 0,19 & $0,67^{*}$ \\
Areia & 0,88 & 0,08 & $-0,82^{*}$ \\
Silte & 0,88 & 0,03 & $0,85^{*}$ \\
DMG & 0,91 & $0,95^{*}$ & 0,11 \\
DMP & 1,00 & $0,99^{*}$ & 0,09 \\
$>2,00 \mathrm{~mm}$ & 1,00 & $0,99^{*}$ & 0,08 \\
$2,00-1,00 \mathrm{~mm}$ & 0,77 & $-0,88^{*}$ & 0,06 \\
$<1,00 \mathrm{~mm}$ & 0,99 & $-0,97^{*}$ & $-0,11$ \\
\hline \multicolumn{4}{c}{ Variância } \\
\end{tabular}

Ds: densidade do solo; MaP: macroporosidade; MiP: microporosidade; VTP: volume total de poros; Ug: umidade gravimétrica; RP: resistência do solo à penetração; $\mathrm{CO}$ : carbono orgânico; EC: estoque de carbono; DMG: diâmetro médio geométrico; DMP: diâmetro médio ponderado; >2,00 mm (\%): classe de agregados maior que 2,00 milímetros; $2,00-1,00 \mathrm{~mm}$ : classe de agregados entre 2,00 e 1,00 milímetros; $<1,00 \mathrm{~mm}(\%)=$ classe de agregados menor que 1 milímetros.

A.

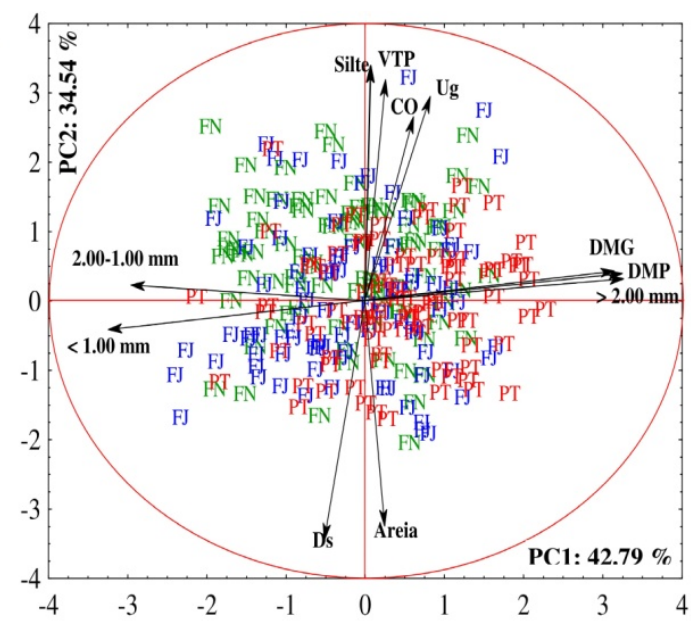

B.

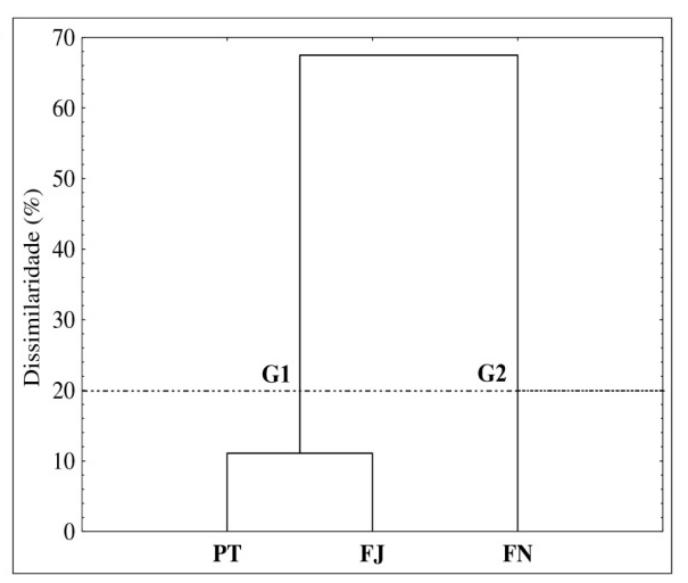

Figura 2. (A) Plano fatorial dos atributos do solo em áreas de Terra Preta Arqueológica sob diferentes usos na região de Novo Aripuanã, Amazonas. Valores padronizados de tal forma que a média é zero e as distancias entre os escores é medido pelo desvio padrão. (B) Dendrograma de agrupamentos dos usos do solo em Terra Preta Arqueológica com linha de corte de $20 \%$, de acordo com os atributos do solo com cargas fatoriais superiores a 0,70 . FN: floresta; FJ: feijão guandu; PT: pastagem. 
Tanto a primeira quanto a segunda fator (Tabela 4) tem percentual de explicação para as características estruturais, mecânica e granulométrica do solo. Assim, a discriminação entre os ambientes diz respeito a essas características, com CP1 representando as características de estabilidade dos agregados do solo (DMG, DMP e classes de agregados $>2,00 \mathrm{~mm}, 2,00-1,00$ $\mathrm{mm}$ e $<1,00 \mathrm{~mm}$ ), enquanto a CP2 às características estruturais do solo, textura e do carbono orgânico (Ds, VTP, Ug, CO, teor de areia e silte).

Na Figura 2A está representado o plano fatorial da distribuição dos escores das diferentes áreas estudadas e da disposição das cargas fatoriais dos atributos do solo formado pelos PC1 e PC2. Observa-se maior adensamento dos escores da floresta no primeiro e segundo quadrante da Figura 2A, o que discrimina a área sob floresta que obtiveram valores dos atributos Ds, VTP, Ug, CO, teor de areia e silte acima da média. Por outro lado, as áreas sob feijão Guandu e pastagem apresentam-se mais distribuído entre o primeiro, terceiro e o quarto quadrante, com atributos que apresentam caracterizados estruturais acima da média, assim como características mecânicas, granulométricas e carbono orgânico abaixo da média.

Utilizando os atributos na análise de agrupamentos (Figura 2B), e considerando a linha de corte $20 \%$ da medida de dissimilaridade, observa-se a formação de dois grupos distintos, com as áreas sob feijão Guandu e pastagem consideradas estatisticamente iguais no que diz respeito ao comportamento dos atributos físicos e carbono orgânico do solo, que é confirmada pela análise fatorial.

Os atributos apresentaram coeficiente de variação (CV) com valores variando de baixo, moderara e alto (Tabelas 1, 2 e 3), segundo os critérios de Warrick e Nielsen (1980), e isso como sendo um indicativo da heterogeneidade dos dados. Verificou-se que em todas as áreas estudadas, e em suas respectivas profundidades, os atributos apresentaram predominância de CV baixo a moderado, corroborando com resultados de Aquino et al. (2015), que avaliaram os atributos físicos em ambientes utilizando o semivariograma escalonado. Também foram verificados comportamentos semelhantes em outros trabalhos com variabilidade espacial dos atributos físicos do solo (Oliveira et al., 2015a; Santos et al., 2012).

Os atributos Ds, MiP, VTP, Ug e teor de areia, apresentaram CV baixo (até $11,63 \%$ ), com exceção a MiP e Ug, que mostraram comportamento de CV mediano sob o uso do feijão Guandu (na camada $0,10-0,20 \mathrm{~m}$ para a MiP e nas três camadas para $\mathrm{Ug}$ ), sendo o último sob o uso de floresta na camada 0,10-0,20 m. Os atributos DMG, DMP e classe de agregados $>2,00 \mathrm{~mm}$, apresentaram $\mathrm{CV}$ de baixo à mediano nas duas primeiras camadas, sob todos os usos do solo, e alta na camada subsequente $(0,10-0,20 \mathrm{~m})$, com exceção do DMG na área de feijão Guandu, que mostrou um CV alto na camada 0,00-0,05 m. Os demais atributos apresentaram CV de mediana a alta em todos os usos e camadas adotadas, sendo a RP com CV todas altas, com valores entre $30 \%$ a $44,90 \%$. Em geral, a área sob floresta foi o que apresentou mais homogeneidade $(\mathrm{CV}=17,93 \%)$, seguido de pastagem $(\mathrm{CV}=21,31 \%)$, ambos com $\mathrm{CV}$ mediano, enquanto que na área sob feijão Guandu, o coeficiente de variação foi de 23,99\%, com alta variabilidade, sendo mais heterogêneo quando comparadas às outras áreas.

Dada a comportamento do CV para os atributos do solo, este pode comparar a variabilidade dos atributos com unidades diferentes. No entanto, para a variabilidade espacial, o semivariograma escalonado foi utilizado, o que observou uma variabilidade espacial na maioria dos atributos, com modelo de ajuste exponencial e esférica (Figuras 3, 4 e 5). 


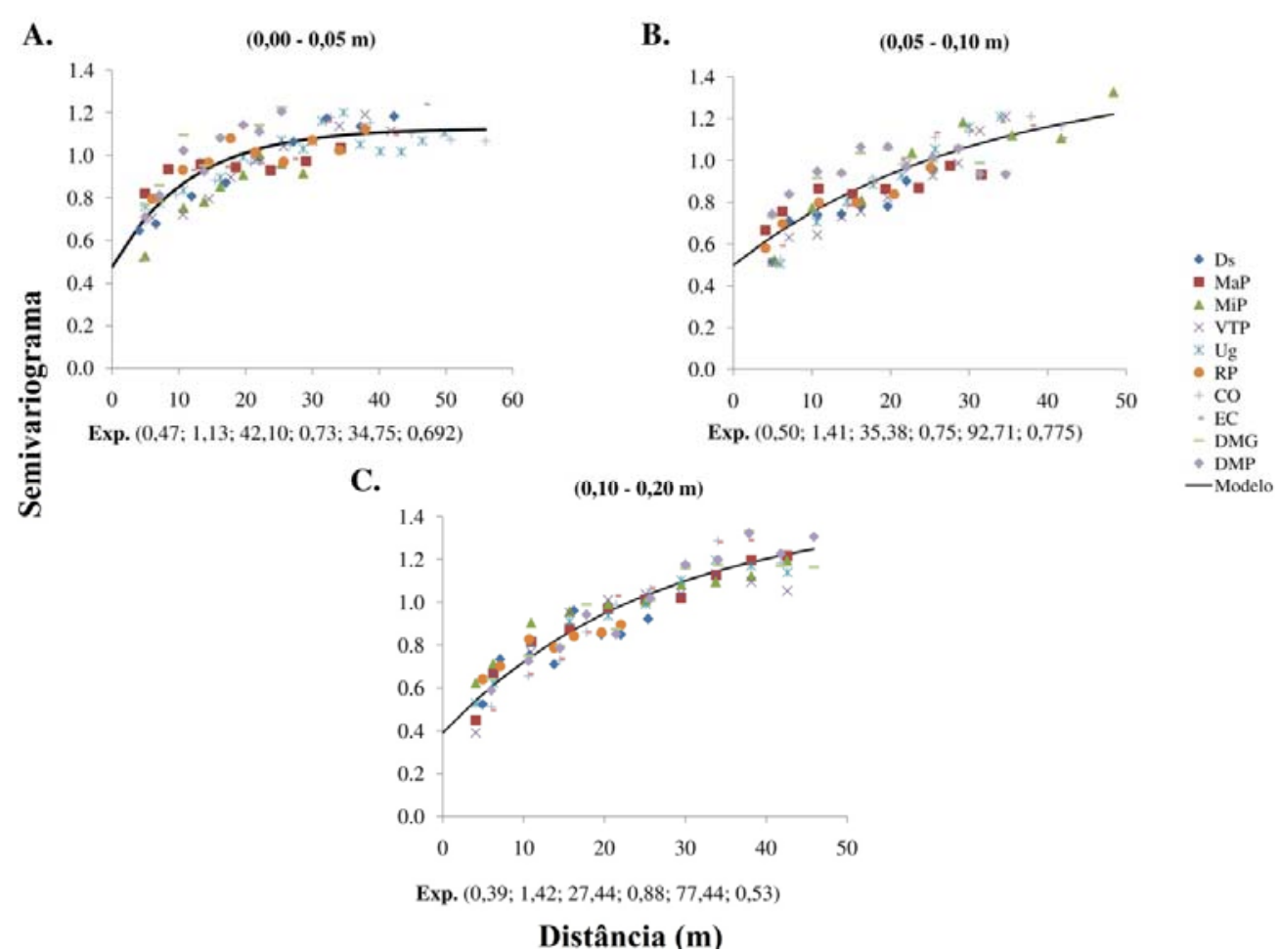

Figura 3. Parâmetros e modelos dos semivariogramas escalonados ajustados aos atributos do solo em áreas de TPA sob floresta na região de Novo Aripuanã, Amazonas. [modelo (efeito pepita; patamar; GDE; R2; alcance; resíduo)]. Esf.: Esférico; Exp.: exponencial; GDE: grau de dependência espacial; $\mathrm{R}^{2}$ : coeficiente de determinação.

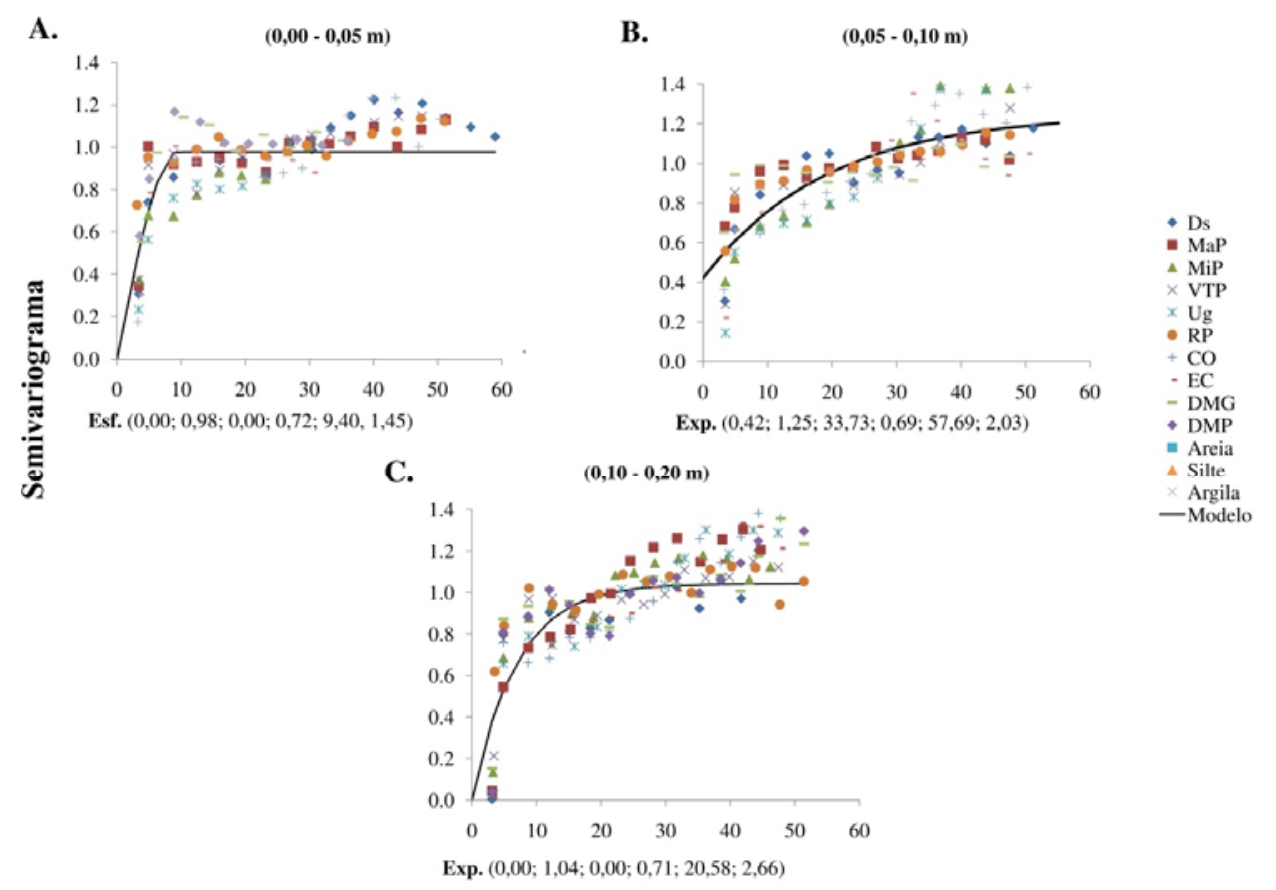

Distância (m)

Figura 4. Parâmetros e modelos dos semivariogramas escalonados ajustados aos atributos do solo em áreas de TPA sob feijão na região de Novo Aripuanã, Amazonas. [modelo (efeito pepita; patamar; GDE; R2; alcance; resíduo)]. Esf.: Esférico; Exp.: exponencial; GDE: grau de dependência espacial; $\mathrm{R}^{2}$ : coeficiente de determinação. 


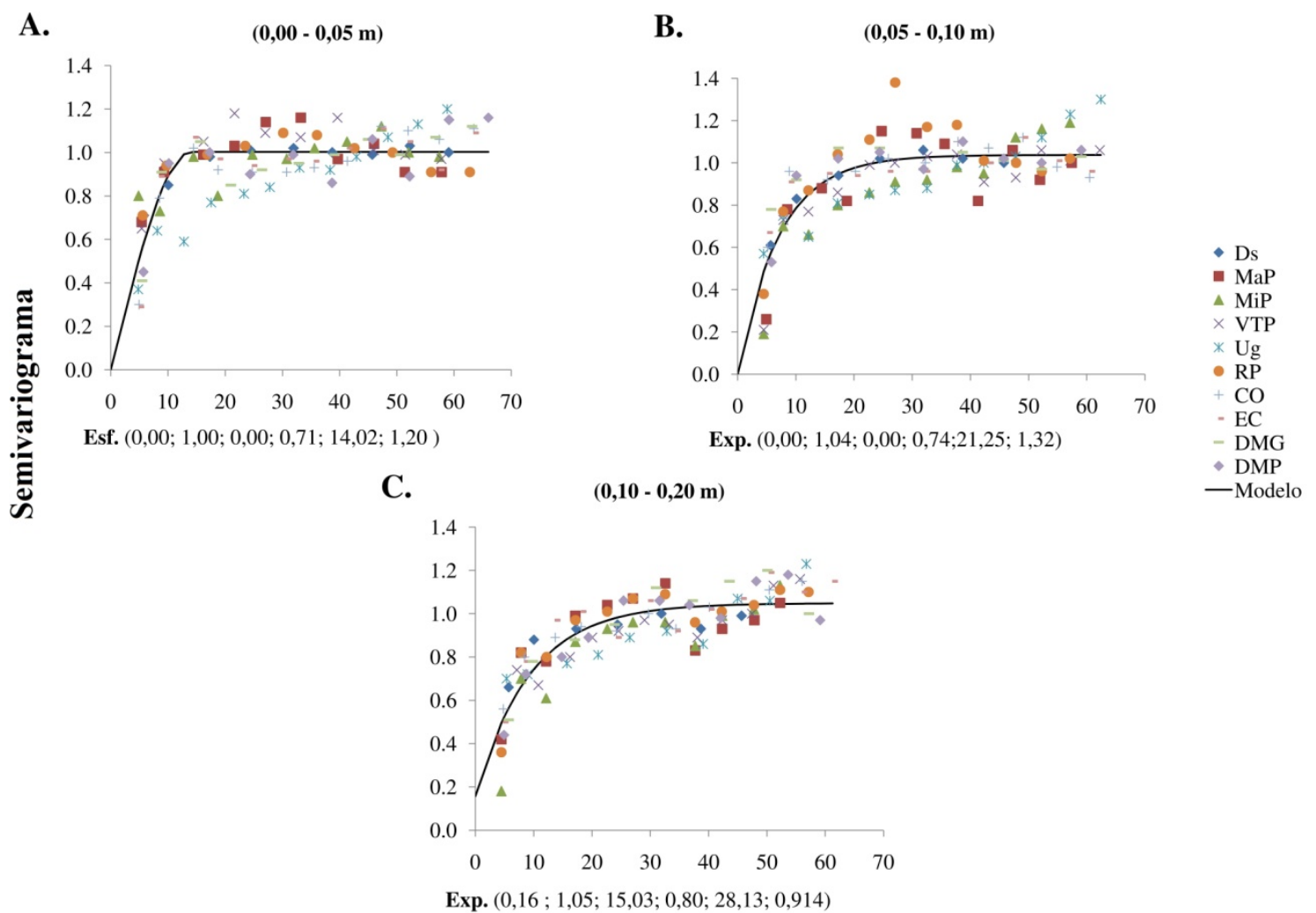

Distância (m)

Figura 5. Parâmetros e modelos dos semivariogramas escalonados ajustados aos atributos do solo em áreas de TPA sob pastagem na região de Novo Aripuanã, Amazonas. [modelo (efeito pepita; patamar; GDE; R2; alcance; resíduo)]. Esf.: Esférico; Exp.: Exponencial; GDE: grau de dependência espacial; $\mathrm{R}^{2}$ : coeficiente de determinação.

Houve mudança de comportamento dos padrões de variabilidade espacial em comparação a área de floresta. O grau de dependência espacial, segundo a classificação de Cambardella et al. (1994), foram fortes em áreas sob feijão Guandu e pastagem nas camadas adotadas (Figuras 4 e 5), com exceção a área de feijão Guandu na camada 0,05-0,10 m, que apresentaram dependência espacial moderada. Por outro lado, a área sob floresta apresentou dependência moderara (Figura 3). Todos esses resultados estão de acordo com os trabalhos de Aquino et al. (2015) e Oliveira et al. (2015a), em se tratando de variabilidade dos atributos do solo em áreas de TPA ou solos adjacentes.

Com base aos ajustes do modelo do semivariograma escalonado, verificou-se comportamento semelhante dos atributos avaliados em relação à área de floresta com as demais áreas. Houve predominância dos modelos exponenciais (Figuras 3, 4 e 5) para o ajuste do semivariograma escalonado, levando em consideração o menor resíduo. O Modelo esférico foi ajustado apenas nas áreas de feijão Guandu e pastagem para a camada de 0,00-0,05 m. Esse resultado corrobora com outras pesquisas, quando avaliado os atributos físicos e químicos em áreas de TPA sob cultivo, onde foram verificados modelos esféricos nas camadas de $0,00-0,10$ $\mathrm{m}$ e de 0,00-0,20 m, respectivamente (Aquino et al., 2015; Oliveira et al., 2015a). O modelo exponencial mostra um comportamento dos atributos com variações mais erráticas (menos contínuas), quando comparadas aos modelos de ajuste esférico, por este apresentar uma variabilidade mais contínua (Isaaks e Srivastava, 1989). Assim, os modelos tiveram bons ajustes ao semivariograma escalonado, com coeficientes de determinação $\left(\mathrm{R}^{2}\right)$ variando entre 0,69 em área de feijão Guandu na camada de 0,05-0,10 m, a 0,88 em área de floresta na camada de $0,10-0,20 \mathrm{~m}$. 
Avaliando os valores do alcance ajustado (Figuras 3, 4 e 5), observou-se que em áreas sob feijão Guandu, com coletas de solos em uma malha com distância mínima de quatro metros, houve menor alcance para a independência da variância entre os pares (patamar), com relação aos atributos do solo, com valores de $9,40 \mathrm{~m}$ e 20,58 $\mathrm{m}$ para as camadas de $0,00-0,05 \mathrm{~m}$ e 0,10 $0,20 \mathrm{~m}$, respectivamente (com exceção à camada de 0,05-0,10 m, que obteve valor de 57,69 m, sendo superior à área sob pastagem para a mesma profundidade). Isso mostra alta variabilidade dos atributos do solo para pequenas distâncias de coleta, fortalecendo a justificativa de maior heterogeneidade dos atributos do solo em relação às demais áreas.

Em área sob pastagem, os alcances foram de 14,02 m, 21,25 e 28,13 m, em suas respectivas camadas, sendo uma malha com distância de coleta mínima de oito (8) metros. Para a área sob floresta, por outro lado, evidenciou um comportamento mais homogêneo para todas as camadas, em comparação as outras áreas, com dependência de correlação espacial chegando a alcance de $34,75 \mathrm{~m}, 92,71 \mathrm{~m}$ e $77,44 \mathrm{~m}$, respectivamente.

\section{CONCLUSÕES}

Houve mudança significativa dos atributos físicos Ds, VTP, MaP e MiP em relação ao tipo de cobertura para camadas até $20 \mathrm{~cm}$ de profundidade. A RP apresentou seu menor valor sob floresta, consistente com os baixos valores de Ug e Ds. Os respectivos usos do solo também promoveram alterações aos valores de $\mathrm{CO}$ e EC, com melhores resultados em áreas sob o uso de feijão guandu e floresta.

$\mathrm{Na}$ análise multivariada, as áreas de feijão guandu e pastagem apresentaram comportamentos semelhantes, com estabilidades dos agregados acima da média, assim como características estruturais, textura e do carbono orgânico abaixo da média.

O modelo exponencial no semivariograma escalonado mostrou grau de dependência espacial forte para os usos do solo sob feijão guandu e pastagem e moderaro para a área sob floresta. A área sob feijão guandu apresentou maior heterogeneidade, com menor alcance de independência espacial da variância entre os pares.

\section{AGRADECIMENTOS}

Os autores agradecem a FAPEAM (Edital 030/2013 - UNIVERSAL AMAZONAS), ao DINTER/CAPES (Edital CAPES 013/2012) e ao Programa de Pós-Graduação em Física Ambiental, pelo financiamento e apoio da pesquisa.

\section{REFERÊNCIAS}

ANDRADE, R.S.; STONE, L.F.; SILVEIRA, P.M. Culturas de cobertura e qualidade física de um Latossolo em plantio direto. Revista Brasileira Engenharia Agrícola e Ambiental, v. 13, p. 411-8, 2009.

AQUINO, R.E.; CAMPOS, M.C.C.; MARQUES JUNIOR, J.; OLIVEIRA, I.A.; TEIXEIRA D.D.B.; CUNHA, J.M. Use of scaled semivariograms in the planning sample of soil physical properties in southern Amazonas, Brazil. Revista Brasileira de Ciência do Solo, v. 39, p. 21-30, 2015. http://dx.doi.org/10.1590/01000683rbes20150524

BRASIL. Ministério das Minas e Energia. Projeto RADAM brasil - Folha SB. 20, Purus. Rio de Janeiro, 1978. 561p.

Rev. Ambient. Água vol. 12 n. 2 Taubaté - Mar. / Apr. 2017 
CALONEGO, J.C.; SANTOS, C.H.; TIRITAN, C.S.; CUNHA JÚNIOR, J.R. Estoque de carbono e propriedades físicas de solos submetidos a diferentes sistemas de manejo. Revista Caatinga, v. 24, p. 128-35, 2012.

CAMBARDELLA, C.A.; MOORMAN, T.B.; NOVAK, J.M.; PARKIN, T.B.; KARLEN, D.L.; TURCO, R.F. et al. Field-scale variability of soil properties in Central Iowa. Soil Science Society of America Journal, v. 58, p. 1501-11, 1994. http://dx.doi.org/10.2136/sssaj1994.03615995005800050033x

CAMPOS, M.C.C.; SANTOS, L.A.C.; SILVA, D.M.P.; MANTOVANELLI, B.C.; SOARES, M.D.R. Caracterização física e química de terras pretas arqueológicas e de solos não antropogênicos na região de Manicoré, AM. Revista Agro@mbiente On-line, v. 6, p. 102-9, 2012. http://dx.doi.org/10.18227/1982-8470ragro.v6i2.682

CEDDIA, M.B.; VIEIRA, S.R.; VILLELA, A.L.O.; MOTA, L.S.; ANJOS, L.H.C.; CARVALHO, D.F. Topography and spatial variability of soil physical properties. Scientia Agrícola, v. 66, p. 338-52, 2009. http://dx.doi.org/10.1590/S010390162009000300009

CRESSIE, N. Statistics for spatial data. New York: John Wiley, 1991.

CUNHA, E.Q.; STONE L.F.; MOREIRA, J.A.A.; FERREIRA, E.P.B.; DIDONET, A.D.; LEANDRO, W.M. Sistemas de preparo do solo e cultura de cobertura na produção orgânica de feijão e milho. Revista Brasileira de Ciência do Solo, v. 35, p. 589-602, 2011.

EMBRAPA. Centro Nacional de Pesquisa do Solo. Manual de métodos de análise de solo. 2. ed. Rio de Janeiro: Embrapa Solo; 2011.

IORI, P.; DIAS JÚNIOR, M.S.; SILVA, R.B. Resistência do solo à penetração e ao cisalhamento em diversos usos do solo em áreas de preservação permanente. Bioscience Journal, v. 28, p. 185-95, 2012.

ISAAKS, E.H.; SRIVASTAVA, R.M. An introduction to applied geoestatistics. New York: Oxford University Press, 1989.

KÄMPF, N.; KERN, D.C. O solo como registro da ocupação humana pré-histórica na Amazônia. In: VIDAL-TORRADO, P.; ALLEONI, L.R.F.; COOPER, M.; SILVA, A.P.; CARDOSO, E.J. (Orgs.). Tópicos em ciência do solo. Viçosa: Sociedade Brasileira de Ciência do Solo, 2005. v. 5. p.277-320.

KEMPER, W.D.; CHEPIL, W.S. Size distribution of aggregates. In: BLACK, C.A.; EVANS, D.D.; WHITE, J.L.; ENSMINGER, L.E.; CLARK, F.E. (Eds.). Methods of soil analysis - Physical and mineralogical properties, including statistics of measurement and sampling. Madison: American Society of Agronomy,1965. p.499-510.

MATOS, E.S.; MENDONÇA, E.S.; LEITE, L.F.C.; GALVÃO, J.C.C. Estabilidade de agregados e distribuição de carbono e nutrientes em Argissolo sob adubação orgânica e mineral. Pesquisa Agropecuária Brasileira, v. 43, p. 1221-30, 2008.

MANLY, B.J.F. Métodos estatísticos multivariados: uma introdução. 3. ed. Porto Alegre: Bookman, 2008.

MELO, V.F.; SCHAEFER, C.E.G.R. Matéria orgânica em solos desenvolvidos de rochas máficas no nordeste de Roraima. Acta Amazonica, v. 39, p. 53-60, 2009. 
OLIVEIRA, I.A.; CAMPOS, M.C.C.; SOARES, M.D.R.; AQUINO, R.E.; MARQUES JUNIOR, J.; NASCIMENTO, E.P. Variabilidade espacial de atributos físicos em um CambissoloHáplico, sob diferentes usos na região do Amazonas. Revista Brasileira de Ciência do Solo, v. 37, p. 1103-12, 2013. http://dx.doi.org/10.1590/S010006832013000400027

OLIVEIRA, I.A.; MARQUES JUNIOR, J.; CAMPOS, M.C.C.; AQUINO, R.E.; FREITAS, L.; SIQUEIRA, D.S. et al. Variabilidade espacial e densidade amostral da suscetibilidade magnética e dos atributos de Argissolos da região de Manicoré, AM. Revista Brasileira de Ciência do Solo, v. 39, p. 668-81, 2015a.

OLIVEIRA, I.A.; CAMPOS, M.C.C.; FREITAS, L.; SOARES, M.D.R. Caracterização de solos sob diferentes usos na região sul do Amazonas. Acta Amazonica, v. 45, n. 1, p. 1-12, 2015b. http://dx.doi.org/10.1590/1809-4392201400555

ROBERTSON, G.P. GS+: Geostatistics for the environmental sciences. Gamma design software. Plainwell: MI, 1998.

ROSSETTI, K.V.; CENTURION, J.F. Estoque de carbono e atributos físicos de um Latossolo em cronossequência sob diferentes manejos. Revista Brasileira de Engenharia Agrícola e Ambiental, v. 19, p. 252-8, 2015. http://dx.doi.org/10.1590/18071929/agriambi.v19n3p252-258

ROZANE, D.E.; CENTURION, J.F.; ROMUALDO, L.M.; TANIGUCHI, C.A.K.; TRABUCO, M.; ALVES, A.U. Estoque de carbono e estabilidade de agregados de um Latossolo Vermelho Distrófico, sob diferentes manejos. Bioscience Journal, v. 26, p. 24$32,2010$.

SANTOS, L.A.C.; CAMPOS, M.C.C.; BERGAMIN, A.C.; SILVA, D.M.P.; MENDONÇA JÚNIOR, A.F. Característica física de seis sitos de terras pretas arqueológicas na região de Apuí-AM. Revista Verde, v. 6, p. 167-74, 2011.

SANTOS, K.S.; MONTENEGRO, A.A.A.; ALMEIDA, B.G.; MONTENEGRO, S.M.G.L.; ANDRADE, T.S.; FONTES JÚNIOR, R.V.P. Variabilidade espacial de atributos físicos em solos do vale aluvial no semiárido de Pernambuco. Revista Brasileira de Engenharia Agrícola e Ambiental, v. 16, p. 828-35, 2012.

SANTOS, L.A.C.; CAMPOS, M.C.C.; AQUINO, R.E.; BERGAMIN, A.C.; SILVA, D.M.P.; MARQUES JUNIOR, J. et al. Caracterização de Terras Pretas Arqueológicas no Sul do Estado do Amazonas. Revista Brasileira de Ciência do Solo, v. 37, p. 825-36, 2013. http://dx.doi.org/10.1590/S0100-06832013000400001

SILVA, A.R.; SILVA, L.L.; FRAZÃO, J.J.; SALGADO, F.H.M.; SILVA, M.C.; CORRECHEL, V. Resistência mecânica à penetração do solo com diferentes coberturas vegetais sob sistema. Revista Cientifica Eletrônica da Agronomia, v. 22, p. x-x, 2012.

SILVA, F.F.; FREDDI, O.S.; CENTURION, J.F.; ARATANI, R.G.; ANDRIOLI, F.F.; ANDRIOLI, I. Propriedades físicas de um Latossolo Vermelho cultivado no sistema plantio direto. Revista Irriga, v. 13, p. 191-204, 2008.

SILVEIRA, D.C.; FILHO, J.F.M; SACRAMENTO, J.A.S.; SILVEIRA, E.C.P. Relação umidade versus resistência à penetração para um Argissolo Amarelo Distrocoeso no Recôncavo da Bahia. Revista Brasileira de Ciência do Solo, v. 34, p. 659-67, 2010. 
SOARES, D. R.; CAMPOS, M. C. C.; SOUZA, Z. M.; BRITO, W. B. M.; FRANCISCON, U.; CASTIONI, G. A. F. Variabilidade espacial dos atributos físicos do solo em área de Terra Preta Arqueológica sob pastagem em Manicoré, AM. Revista de Ciências Agrárias, v. 58, n. 4, p. 434-441, 2015.

STATSOFT INC. Statistica - data analysis software system. Tulsa, 2004.

TAVARES FILHO, J.; TESSIER, D. Compressibility of oxisol aggregates under no-till in response to soil water potential. Revista Brasileira de Ciência do Solo, v. 33, n. 6, p. 1525-1533, 2009. http://dx.doi.org/10.1590/S0100-06832009000600002

VIANA, E.T.; BATISTA, M.A.; TORMENA, C.A.; COSTA, A.C.S.; INOUE, T.T. Atributos físicos e carbono orgânico em Latossolo Vermelho sob diferentes sistemas de uso e manejo. Revista Brasileira de Ciência do Solo, v. 35, p. 2105-14, 2011.

VOLK, L.B.S.; COGO, N.P. Relações entre tamanho de sedimentos erodidos, velocidade da enxurrada, rugosidade superficial criada pelo preparo e tamanho de agregados em solo submetido a diferentes manejos. Revista Brasileira de Ciência do Solo, v. 33, p. 1459$71,2009$.

WARRICK, A.W.; NIELSEN, D.R. Spatial variability of soil physical properties in the field. In: HILLEL, D. (Ed.). Applications of soil physics. New York: Academic Press, 1980. p.319-44.

WHITE, P.J.; CRAWFORD, J.W.; ÁLVAREZ, M.C.D.; MORENO, R.G. Soil Management for Sustainable Agriculture. Applied and Environmental Soil Science, v. 2012, p. 1-3, 2012. http://dx.doi.org/10.1155/2012/850739

YEOMANS, J.C.; BREMNER, J.M. A rapid and precise method for routine determination of organic carbon in soil. Communications in Soil Science and Plant Analysis, v. 19, p. 1467-76, 1988. http://dx.doi.org/10.1080/00103628809368027 


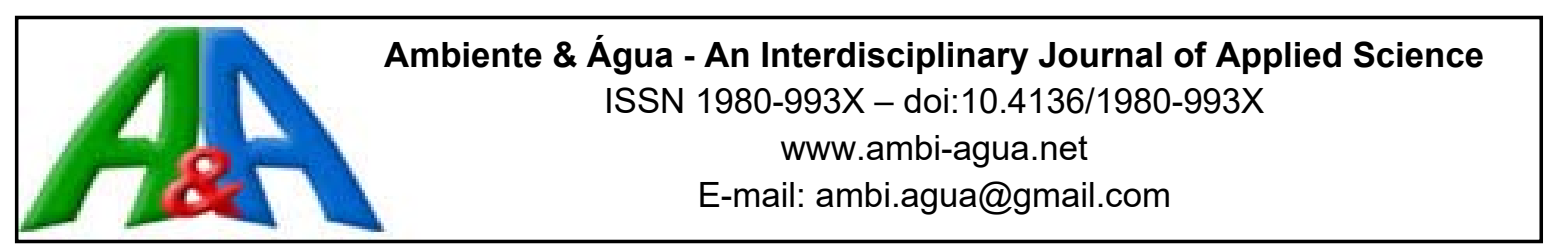

\title{
Diagnóstico espacial e temporal de condições físico-químicas e microbiológicas do Córrego do Tanquinho, Ribeirão Preto, SP, Brasil
}

\author{
doi:10.4136/ambi-agua.1837
}

Received: 09 Jan. 2016; Accepted: 31 Jan. 2017

\author{
Andre Abreu Grieco' ${ }^{1}$; Brisa Maria Fregonesi ${ }^{1}$; \\ Karina Aparecida de Abreu Tonani ${ }^{1}$; Thaís Vilela Silva ${ }^{1}$; \\ Beatriz Smidt Celere ${ }^{1}$; Tânia Maria Beltramini Trevilato ${ }^{2}$; \\ Susana Inés Segura-Muñoz ${ }^{1}$; Renato Igor da Silva Alves ${ }^{1 *}$ \\ Universidade de São Paulo (USP), Ribeirão Preto, SP, Brasil \\ Escola de Enfermagem de Ribeirão Preto (EERP) \\ ${ }^{1}$ Departamento de Enfermagem Materno-Infantil e Saúde Pública \\ ${ }^{2}$ Setor de Metais do Hospital das Clínicas \\ *Autor correspondente: e-mail: renatobio_usp@yahoo.com.br, \\ andregrieco11@gmail.com, brisa fregonesi@yahoo.com.br, \\ karina_abreustz@yahoo.com.br, thaiis_vilela@hotmail.com, \\ beacelere@gmail.com,tmbeltra@fmrp.usp.br, susis@eerp.usp.br
}

\section{RESUMO}

O objetivo do estudo foi avaliar as condições fisico-químicas e microbiológicas do Córrego do Tanquinho, localizado em área altamente urbanizada e industrializada no município de Ribeirão Preto, SP, Brasil, considerando variações espaciais e temporais. Os parâmetros limnológicos avaliados foram: $\mathrm{pH}$, temperatura, turbidez, condutividade e oxigênio dissolvido (OD), com medições realizadas in situ. Os níveis de metais no sedimento e em água superficial foram analisados por Espectrofotometria de Absorção Atômica. Para a determinação dos Coliformes Totais e Escherichia coli foi empregada a técnica dos Tubos Múltiplos com utilização de substrato cromogênico. Os parâmetros OD, E. coli e a concentração dos metais $\mathrm{Cu}$ e $\mathrm{Mn}$ na água encontram-se em desacordo com a legislação. Os metais encontrados em maior concentração no sedimento foram $\mathrm{Mn}, \mathrm{Cr}$ e $\mathrm{Zn}$. A avaliação microbiológica evidenciou o despejo de esgoto doméstico no córrego e as concentrações de metais indicam a possível ocorrência de efluentes industriais, podendo gerar graves problemas de saúde pública. $\mathrm{O}$ diagnóstico espacial indicou os locais de amostragem com as piores condições microbiológicas e altas concentrações de metais, enquanto que a influência temporal foi observada no $\mathrm{pH}, \mathrm{Mn}$ e Cr em água superficial, e $\mathrm{Mn}, \mathrm{Cr}$ e $\mathrm{Pb}$ nos sedimentos. Os resultados mostraram que o Córrego do Tanquinho é um corpo hídrico impactado. Recomenda-se a realização de outros estudos que possam esclarecer melhor a origem das cargas poluidoras, além da conscientização da população e da adoção de medidas que possibilitem a recuperação e proteção desse corpo hídrico.

Palavras-chave: água superficial, E. coli, metais, parâmetros limnológicos, sedimento. 


\title{
Spatial and temporal diagnosis of the physicochemical and microbiological conditions of Tanquinho's Stream, Ribeirão Preto, SP, Brazil
}

\begin{abstract}
The aim of the study was to evaluate the physico-chemical and microbiological conditions of Tanquinho's Stream, located in a highly urbanized and industrialized area in Ribeirão Preto, SP, Brazil, taking into account spatial and temporal variations. The following limnological parameters were evaluated: $\mathrm{pH}$, temperature, turbidity, conductivity and dissolved oxygen (DO), with measurements in situ. Metal levels in sediment and surface water were analyzed by Atomic Absorption Spectrometry. The Multiple Tubes technique with chromogenic substrate was used to determine Total Coliforms and Escherichia coli. The parameters of DO, E. coli and the concentration of $\mathrm{Cu}$ and $\mathrm{Mn}$ in the water are not in accordance with national legislation. The metals found in higher concentrations in the sediment were $\mathrm{Mn}, \mathrm{Cr}$ and $\mathrm{Zn}$. The microbiological evaluation showed sewage discharges into the stream and the metal concentrations indicate possible industrial effluents, which may cause serious public health problems. The spatial diagnosis indicated the sampling sites with the worst microbiological conditions and high concentrations of metals, while the temporal influence was observed in the $\mathrm{pH}, \mathrm{Mn}$ and $\mathrm{Cr}$ in surface water, and $\mathrm{Mn}, \mathrm{Cr}$ and $\mathrm{Pb}$ in the sediments. The results indicate that Tanquinho's Stream is an impacted water body. It is recommended that further studies be conducted to clarify the source of pollutants and to assess the degree of public awareness, and that measures be adopted to ensure the recovery and protection of this water body.
\end{abstract}

Keywords: E. coli, metals, limnology parameters, sediment, surface water.

\section{INTRODUÇÃO}

Apesar da grande disponibilidade, a água doce no Brasil não está distribuída de maneira homogênea pelo território nacional. A Região Sudeste concentra $42,65 \%$ da população do país e apenas $6 \%$ dos recursos hídricos, enquanto que a Região Norte abriga 68,50\% dos recursos hídricos e apenas 6,98\% da população (Philippi Jr. e Martins, 2005).

O desenvolvimento das cidades e das atividades humanas podem causar prejuízos significativos para a sociedade, principalmente no que diz respeito à qualidade da água (Mei et al., 2014). Somado à intensa urbanização e à carência de esgotamento sanitário, o processo de industrialização do país contribuiu para um aumento significativo da carga poluidora lançada nos corpos d'água presentes em áreas urbanas (Souza e Andrade, 2014).

O município de Ribeirão Preto está localizado na região Nordeste do Estado de São Paulo e a maior parte do seu território situa-se na Bacia Hidrográfica do Rio Pardo. A bacia do Rio Pardo, cuja drenagem é da ordem de $10.849 \mathrm{~km}^{2}$, abriga um contingente populacional de cerca de 1.108.118 habitantes, sendo Ribeirão Preto o município mais populoso. Caracterizado como um dos mais importantes polos regionais do estado, o município vive um intenso desenvolvimento econômico, com destaque para indústria sucroalcooleira e, mais recentemente, no segmento de alta tecnologia, fator impulsionador do desenvolvimento técnico-científico da região (Ribeirão Preto, 2012).

Os córregos que cortam a cidade de Ribeirão Preto apresentam inúmeras irregularidades, como matas ciliares degradadas e pouca área mínima de recuo. Os corpos d'água também recebem cargas clandestinas de esgoto, além do lixo carregado pelas chuvas ou depositado pela população (Da Silva Alves et al., 2013). O Córrego do Tanquinho, objeto deste estudo, está localizado em área urbana do município de Ribeirão Preto. O córrego possui 7.048,02 m de 
comprimento e bacia hidrográfica de $20,37 \mathrm{~km}^{2}$ de área, densamente povoada e ocupada por notáveis bairros industriais da cidade (Tomazelli et al., 2010).

A hidrobiologia e qualidade da água do Córrego do Tanquinho podem estar comprometidas considerando sua localização no perímetro urbano. Sabe-se que as características naturais dos cursos d'água são alteradas pelo lançamento irregular de efluentes domésticos, erosão das margens e por resíduos depositados diretamente pela população (Purandara et al., 2012).

Nesse cenário de urbanização, sem um correto planejamento ambiental e infraestrutura adequada, acentua-se a diminuição e escassez de recursos hídricos, motivo pelo qual é necessário que se realizem estudos de avaliação e monitoramento da qualidade da água. Tais estudos proveem indicadores e informações que oferecem respaldo para a tomada de decisão com relação ao planejamento ambiental e proteção dos recursos hídricos realizados pelos órgãos de gestão responsáveis. Esses estudos e análises que permitem verificar as concentrações dos poluentes, além das possíveis fontes das cargas poluidoras, são fundamentais para elaboração de políticas de controle de poluição. Recentemente, tais estudos têm ganhado relevância no Brasil e no mundo (Shin et al., 2013).

Em vista disso, o objetivo do presente estudo foi avaliar as condições fisico-químicas e microbiológicas do Córrego do Tanquinho, localizado em uma área altamente urbanizada e industrializada no município de Ribeirão Preto - SP, considerando variações espaciais e temporais.

\section{MATERIAL E MÉTODOS}

\subsection{Local de estudo e coleta de amostras}

O presente trabalho foi desenvolvido no córrego do Tanquinho (Figura 1), cuja nascente está localizada nas proximidades do Parque Industrial Lagoinha, na Região Leste do Município de Ribeirão Preto, SP, Brasil. O córrego cruza os bairros Jardim Anhanguera, Parque dos Bandeirantes, Jardim Paulistano, Jardim Independência, Campos Elíseos, Parque Industrial Tanquinho e deságua no córrego Ribeirão Preto na região norte da cidade. Dessa forma, sua bacia é caracterizada pela alta urbanização, com a presença de bairros populosos e industriais.

Foram selecionados cinco pontos para as coletas das amostras. Não foi possível a coleta na nascente do córrego, já que a mesma se encontra canalizada e sem acesso. O ponto 1 foi escolhido pois é o primeiro local em que é possível ter acesso ao leito do córrego a partir de sua nascente e localiza-se muito próximo a ela. Neste trecho o córrego já se encontra canalizado, ainda assim sua caracterização se destaca dos demais locais de coleta: as margens são mais preservadas por vegetação; a distância para residências e construções é maior; a profundidade, o leito, o fluxo e volume de água são menores que nos demais pontos. É importante salientar que esses parâmetros não foram mensurados e são baseados nas observações realizadas em visitas aos locais.

Os demais locais de coleta, ponto 2 , ponto 3 , ponto 4 e ponto 5 se caracterizam de forma muito semelhante: ausência de vegetação nas margens; proximidade às residências e construções; presença de resíduos sólidos nas margens e leito; alterações antrópicas no leito; fluxo, volume de água e profundidade similares. A seleção destes pontos foi baseada nas possíveis descargas de efluentes, proximidade aos principais bairros, maior equidistância entre os pontos, foz (confluência com o córrego Ribeirão Preto) e facilidade de acesso.

Foram realizadas duas campanhas para coleta das amostras: no período seco e no período chuvoso (setembro, 2013 e fevereiro, 2014). Nas duas campanhas as amostras foram coletadas em um único dia no período matutino, entre as $08 \mathrm{~h} 00$ e $12 \mathrm{~h} 00$. 


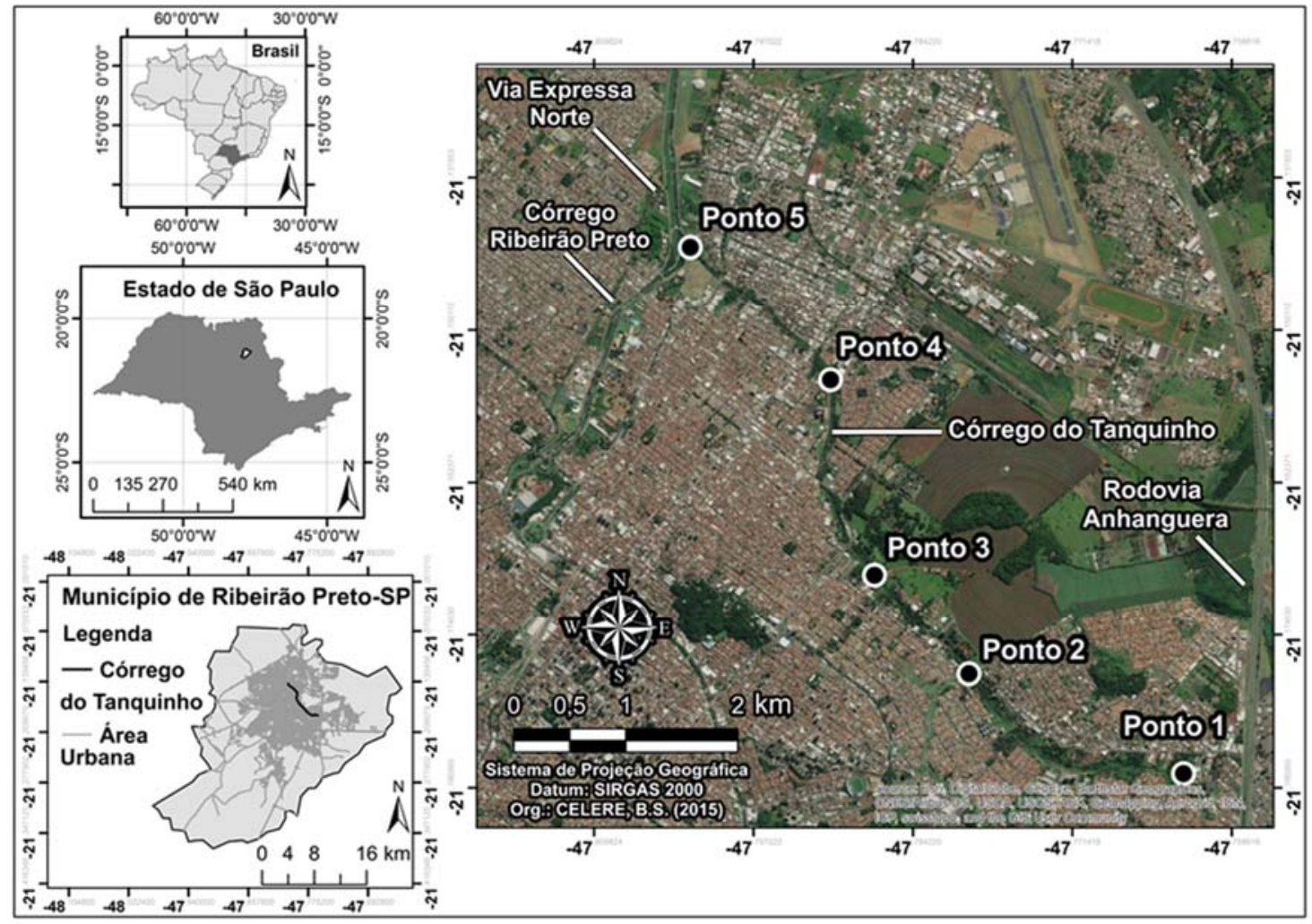

Figura 1. Córrego do Tanquinho destacando os cinco pontos de amostragem, no município de Ribeirão Preto, SP, Brasil.

\subsection{Parâmetros limnológicos}

As medições dos parâmetros limnológicos foram realizadas in situ. As amostras de água foram coletadas em balde de aço inox previamente ambientado no local e analisadas imediatamente.

As leituras de $\mathrm{pH}$ foram executadas com medidor de $\mathrm{pH}$ (marca PH-TEK, modelo $\mathrm{pH} 100$ ). As leituras de temperatura foram executadas com termômetro digital (marca MINIPA, modelo MV365). A turbidez foi determinada utilizando um turbidímetro portátil (marca HANNA ${ }^{\circledR}$, modelo HI 93703). A medição da condutividade foi realizada utilizando um condutivímetro (marca LT Lutron, modelo CD-4303). O teor de oxigênio dissolvido (OD) foi mensurado por meio de um oxímetro (marca Lutron, modelo DO-5510).

Foi registrada uma medição para cada parâmetro em todos os pontos de coleta nas duas campanhas realizadas. Outras medições não registradas foram realizadas apenas para estabilização e/ou calibração dos equipamentos conforme instrução dos manuais.

\subsection{Metais}

Os recipientes utilizados na coleta e armazenamento de amostras eram de polietileno e foram previamente submergidos em solução de ácido nítrico a $30 \%$ por $24 \mathrm{~h}$ para a eliminação de metais interferentes (APHA et al., 2012). As amostras de água foram retiradas da superfície (entre 0 e $30 \mathrm{~cm}$ da lâmina d'água) com auxílio de balde de aço inox previamente ambientado no local, ou, quando possível, coletadas diretamente em recipiente de polietileno de $50 \mathrm{~mL}$ utilizados para preservação e armazenamento. As amostras de sedimento foram coletadas com Draga de Eckman e mantidas à temperatura ambiente por 30 dias em caixas de polietileno, no Laboratório de Ecotoxicologia e Parasitologia Ambiental da Escola de Enfermagem de Ribeirão Preto (EERP)/USP. 
Nas amostras de águas superficiais, adicionou-se ácido nítrico de alta pureza (65\% Suprapur-Merk, Germany) para a fixação dos metais $(\mathrm{pH}<2)$. As amostras foram mantidas a $-18^{\circ} \mathrm{C}$ até o momento da dosagem (APHA et al., 2012).

Para a análise das amostras de sedimento utilizou-se Água Régia ( $\mathrm{HNO}_{3}$; HCL; 3:1). Aproximadamente $0,5 \mathrm{~g}$ de cada amostra foram colocados em bombas de teflon e adicionados $10 \mathrm{ml}$ de Água Régia. As bombas de teflon foram vedadas, deixadas em repouso por $8 \mathrm{~h}$ à temperatura ambiente e posteriormente digeridas a $80^{\circ} \mathrm{C}$ durante $8 \mathrm{~h}$. Após o término da digestão as amostras foram filtradas (Whatman Grade No 40) e diluídas a $25 \mathrm{ml}$ com água Milli-Q (MILLIPORE Direct-Q ${ }^{\circledR} 3$ UV).

Após o pré-tratamento, as amostras de sedimento e água superficial foram encaminhadas para o Setor de Metais do Hospital das Clínicas da Faculdade de Medicina de Ribeirão Preto (FMRP/USP), para as dosagens de Cromo $(\mathrm{Cr})$, Chumbo $(\mathrm{Pb})$, Cobre $(\mathrm{Cu})$, Manganês $(\mathrm{Mn})$, Cádmio $(\mathrm{Cd})$ e Zinco $(\mathrm{Zn})$. As dosagens de $\mathrm{Cr}, \mathrm{Pb}, \mathrm{Mn}$ e $\mathrm{Cd}$ foram realizadas por Espectrofotometria de Absorção Atômica com Forno de Grafite (EAA- FG) em um Espectrofotômetro VARIAN - ZEEMAN (Modelo 640-Z). As dosagens de Zn e Cu foram realizadas por Espectrofotometria de Absorção Atômica de Chama (EAA - chama) em um Espectrofotômetro VARIAN, (Modelo AA-200).

Foram utilizados padrões certificantes de água não potável (NW 556; NW 567; NW 572) do Instituto Quality Control Technologies Pty Ltd., Queensland, Austrália.

\subsection{Análise microbiológica}

A coleta e análise das amostras para avaliação de coliformes totais e Escherichia coli foram executadas de acordo com APHA et al. (2012) e da Companhia Ambiental do estado de São Paulo - CETESB (2011), utilizando a técnica dos Tubos Múltiplos com utilização de substrato cromogênico definido $\left(\right.$ Colilert $\left.^{\circledR}\right)$. Os resultados foram obtidos em Número Mais Provável em $100 \mathrm{~mL}\left(\mathrm{NMP} \mathrm{mL}^{-1}\right)$.

\subsection{Análise dos dados}

Os resultados obtidos com as avaliações limnológicas, microbiológicas e dos metais pesados em água superficial foram comparados com os valores máximos permissíveis da Resolução CONAMA N N $^{357 / 2005}$ que classifica os corpos de água em 5 classes principais, considerando o uso da água e o risco para a saúde pública: classe especial, classe 1, classe 2, classe 3, classe 4 (CONAMA, 2005). De acordo com essa legislação o Córrego do Tanquinho é classificado como classe 2. As águas da classe 2 são destinadas ao abastecimento para consumo humano, após tratamento convencional; à proteção das comunidades aquáticas; à recreação de contato primário, tais como natação, esqui aquático e mergulho; à irrigação de hortaliças, plantas frutíferas e de parques, jardins, campos de esporte e lazer, com os quais o público possa vir a ter contato direto; e à aquicultura e à atividade de pesca. Visto que o Brasil não estabelece valores máximos permissíveis para sedimento em rios e córregos, foram considerados parâmetros internacionais para análise de sedimento. A Resolução CONAMA $n^{\circ} 344 / 2004$, a qual estabelece as diretrizes gerais do material dragado em águas jurisdicionais brasileiras (CONAMA, 2004), também foi utilizada na análise dos dados.

A análise estatística foi realizada por meio do software SPSS Statistics Base 17.0. Os dados que apresentaram distribuição normal (paramétricos) foram submetidos ao teste $t$ de Student para verificar se houve diferença estatística significativa $(\mathrm{p} \leq 0,5)$ entre as duas campanhas de coleta. Já os dados que não apresentaram distribuição normal (não paramétricos) foram submetidos ao Teste de Mann-Whitney. O Teste r- Spearman foi utilizado para verificar correlações entre os parâmetros analisados em água $(p \leq 0,05)$. As concentrações de metais que 
apresentaram valores abaixo do limite de detecção (LD) foram consideradas como metade do valor do limite de detecção (LD/2) para realização dos cálculos.

\section{RESULTADOS E DISCUSSÃO}

\subsection{Parâmetros limnológicos}

Os resultados obtidos na avaliação dos parâmetros limnológicos: $\mathrm{pH}$, temperatura, turbidez, condutividade e OD nos períodos seco e chuvoso estão apresentados na Tabela 1.

Tabela 1. Parâmetros limnológicos em amostras de água do Córrego do Tanquinho, Ribeirão Preto, SP, Brasil, durante os períodos seco e chuvoso.

\begin{tabular}{cccccc}
\hline \multicolumn{5}{c}{ Período Seco } \\
\hline Pontos de Coleta & $\mathrm{pH}$ & $\begin{array}{c}\text { Temperatura } \\
\left({ }^{\circ} \mathrm{C}\right)\end{array}$ & $\begin{array}{c}\text { Turbidez } \\
(\mathrm{FTU})\end{array}$ & $\begin{array}{c}\text { Condutividade } \\
\left(\mu \mathrm{S} \mathrm{cm}^{-1}\right)\end{array}$ & $\begin{array}{c}\text { Oxigênio Dissolvido } \\
\left(\mathrm{mg} \mathrm{L}^{-1}\right)\end{array}$ \\
\hline 1 & 7,0 & 21,3 & 5,6 & 128,5 & $3,3^{*}$ \\
2 & 6,9 & 22,2 & 11,5 & 213 & $3,2^{*}$ \\
3 & 7,0 & 24,4 & 6,5 & 197 & $4,8^{*}$ \\
4 & 6,8 & 26 & 14,9 & 224 & 5,6 \\
5 & 6,6 & 26,8 & 7,1 & 247 & $3,2^{*}$ \\
Média & 6,8 & 24,1 & 9,1 & 201,9 & 4,0 \\
DP & 0,2 & 2,4 & 3,9 & 44,9 & 1,1 \\
Máximo & 7,0 & 26,8 & 14,9 & 247 & 5,6 \\
Mínimo & 6,6 & 21,3 & 5,6 & 128,5 & 3,2 \\
\hline & & & Período Chuvoso & & 4,9 \\
\hline 1 & 6,5 & 24,3 & 1,5 & 135 & $4,8^{*}$ \\
2 & 6,2 & 24,8 & 12,9 & 186,8 & $4,9^{*}$ \\
3 & 6,2 & 26,4 & 11,0 & 185,3 & $4,0^{*}$ \\
4 & 6,2 & 28,8 & 23,1 & 214 & 4,7 \\
5 & 6,4 & 29,9 & 6,1 & 227 & 0,8 \\
Média & 6,3 & 26,7 & 10,9 & 189,6 & 5,9 \\
DP & 0,1 & 2,6 & 8,1 & 35,4 & 4,0 \\
Máximo & 6,5 & 29,8 & 23,1 & 227 & 135 \\
Mínimo & 6,2 & 24,2 & 1,5 & - & \\
VMP & $6,0 \mathrm{a} 9,0$ & - & $\leq 100$ & 5,0 & \\
\hline
\end{tabular}

VMP: Valor máximo permitido pela resolução CONAMA nº 357/2005 para águas classe 2;

-: Não há VMP estabelecido;

*: Valor em desacordo com o VMP.

Os valores de $\mathrm{pH}$ encontram-se dentro dos limites estabelecidos pela Resolução CONAMA $\mathrm{n}^{\mathrm{o}} 357 / 2005$. Comparando os valores de $\mathrm{pH}$ dos períodos seco e chuvoso, foi encontrada diferença estatística significativa $(\mathrm{p}<0,05)$, onde foi observado redução do $\mathrm{pH}$ no período chuvoso. Esse resultado é condizente com os trabalhos de Purandara et al. (2012) e Bayram et al. (2013), e pode ser explicado pelo aumento de matéria orgânica e de outros resíduos carreados pelas chuvas. Além disso, o período chuvoso ocorre no verão, e à medida que a temperatura aumenta ocorre a ionização da água, o que estimula a concentração de íons de hidrogênio e a 
redução no pH (Bayram et al., 2013). Entretanto, essa relação entre pH e temperatura foi melhor observada no período seco onde foi encontrada correlação negativa $(r=-0,821)$ significativa $(\mathrm{p}<0,05)$ entre $\mathrm{pH}$ e temperatura.

A variação da temperatura ao longo do córrego foi similar nos dois períodos. Isso se deve ao fato de que as campanhas de coleta foram realizadas em horários muito semelhantes. A temperatura ambiente aumentava ao decorrer da coleta, refletindo a variação diária da temperatura. Como mostrado na Tabela 1, a condutividade apresentou menor valor no ponto 1 , aumentando de maneira constante ao longo do córrego, com exceção do ponto 2 que, nos dois períodos, foi maior que o ponto 3. Sabe-se que o aumento de condutividade ao longo do rio pode estar relacionado ao acúmulo de cargas poluidoras lançadas por efluentes, ao aporte de cargas poluidoras difusas provenientes do escoamento superficial, e ao acúmulo de íons e sólidos dissolvidos (Purandara et al., 2012). A ausência de vegetação nas margens observada no córrego facilita o aporte de materiais por escoamento superficial.

Foi observada correlação positiva $(\mathrm{r}=0,900)$ e significativa $(\mathrm{p}<0,05)$ entre temperatura e condutividade em ambos os períodos. $\mathrm{O}$ aumento da temperatura pode promover o aumento de sólidos dissolvidos e alguns íons dissociados, que podem elevar a condutividade (Wilbers et al., 2014). Também foi constatada correlação negativa $(r=-0,975)$ significativa $(p<0,05)$ entre condutividade e $\mathrm{pH}$, mas apenas no período seco. Isso pode estar relacionado ao aumento de íons dissociados ao longo do córrego, como íons $\mathrm{H}+$, que podem promover simultaneamente o aumento da condutividade e a redução do $\mathrm{pH}$.

Os valores de turbidez encontrados no córrego do Tanquinho encontram-se dentro do limite estabelecido pela legislação. O aumento da turbidez é decorrente de elevadas cargas de material em suspensão, as quais estão associadas ao lançamento de esgotos domésticos e industriais e ao aporte de cargas difusas provenientes principalmente do escoamento superficial (CESTESB, 2012; Siqueira et al., 2012). Os maiores níveis de turbidez encontrados nos pontos 2 e 4, coincidem com as maiores quantidades de Coliformes Totais e E. coli encontradas (Tabela 2). Isso evidencia a relação direta entre o aumento da turbidez e a provável grande quantidade de efluentes sanitários na água que é indicada pela elevada presença de Coliformes Totais e E. coli. Essa relação é corroborada pela correlação positiva significativa $(\mathrm{p}<0,05)$ observada entre a turbidez e Coliformes Totais (seca: $r=0,975$; chuva: $r=0,900$ ) e a E. coli (seca e chuva: $r=0,900)$.

Como observado na Tabela 1 , apenas o ponto 4 (período seco) e o ponto 1 (período chuvoso) apresentaram OD acima de $5 \mathrm{mg} \mathrm{L}^{-1}$, estando os outros pontos em desacordo com a legislação (CONAMA, 2005). Diminuições nas concentrações de OD em água estão diretamente relacionadas ao aumento da quantidade de matéria orgânica proveniente de efluentes domésticos, industriais e fontes difusas (Alvani et al., 2011). Dessa maneira, poderíamos esperar que o ponto 1 , devido às suas características já descritas, apresentasse os maiores valores de OD, o que foi observado apenas no período chuvoso. Uma das explicações para os baixos valores de OD no ponto 1 pode estar relacionada a baixa vazão e fluxo de água no local, principalmente no período seco, que embora não tenham sido mensuradas, essas características podem estar presentes no ponto 1 como já mencionado. A velocidade da correnteza exerce influência sobre a concentração de OD na água, uma vez que o aumento do fluxo de água leva ao aumento da turbulência, da aeração e, consequentemente, da solubilidade do oxigênio (Krupek et al., 2008).

Embora o período chuvoso tenha apresentado maior concentração de OD em quatro dos cinco pontos de coleta, não houve diferença estatística significativa $(p>0,05)$ entre os valores dos dois períodos de coleta. Valores maiores de OD podem ser esperados no período chuvoso já que o maior fluxo e volume de água promovem diluição da matéria orgânica, aeração e 
aumento da turbulência, como já observado por Tomazelli et al. (2010), no córrego do Tanquinho, e por Purandara et al. (2012).

Foi observada no período chuvoso uma correlação negativa $(\mathrm{r}=-1,000)$ e significativa $(\mathrm{p}<0,05)$ entre o OD e a condutividade, evidenciando a presença de fatores que podem, simultaneamente, promover o aumento da condutividade e a diminuição do OD. Grande quantidade de matéria orgânica pode ser um fator causador dessa correlação, já que está relacionada à diminuição do oxigênio dissolvido (Alvani et al., 2011) e ao aumento da condutividade (Purandara et al., 2012).

\subsection{Análise microbiológica}

A Tabela 2 mostra os resultados da análise de coliformes totais e E. coli nos períodos seco e chuvoso.

O grupo de bactérias coliformes está entre os principais micro-organismos utilizados como indicadores de poluição de origem fecal e é utilizado de forma universal na verificação de presença ou ausência de agentes patogênicos na água e no estabelecimento de padrões de qualidade (WHO, 2011; Wilbers et al., 2014). Dessa forma, os valores de coliformes totais encontrados no Córrego do Tanquinho indicam que esse corpo hídrico possui elevada carga de poluição microbiológica, com destaque para os pontos 2 e 4 (Tabela 2), possivelmente proveniente de descargas irregulares de esgoto doméstico. Porém, os coliformes totais incluem gêneros que não são de origem exclusivamente fecal, como Citrobacter, Enterobacter $e$ Klebsiela, e que podem se desenvolver na água, em solos e plantas, o que limita sua utilização como indicador específico de contaminação fecal (WHO, 2011). Assim, o escoamento superficial e a lavagem do solo pelas chuvas podem contribuir nos valores de coliformes totais no Córrego do Tanquinho. No entanto, é provável que o lançamento de efluentes contribua com a maior parte dos coliformes totais encontrados no córrego, uma vez que foram encontradas quantidades elevadas de E. coli, o que será abordado no próximo item da discussão. Além disso, o valor encontrado nos pontos 2 e $4\left(1,30 \mathrm{E}+07 \mathrm{NMP} 100 \mathrm{~mL}^{-1}\right)$ se aproximam às concentrações encontradas por Tonani (2011) em amostras de esgoto bruto (in natura) da Estação de Tratamento de Esgoto de Ribeirão Preto - ETE/RP. Em duas das amostras analisadas por Tonani (2011) foram encontrados 1,30E+07 de NMP $100 \mathrm{~mL}^{-1}$ de coliformes totais, mesmo valor encontrado no ponto 2 em ambos os períodos, e no ponto 4 no período seco. A presença de elevadas quantidades de coliformes totais é comum em rios que passam por locais densamente urbanizados, como destacado por Wilbers et al. (2014).

Comparando os valores encontrados durante os dois períodos, não foi observada diferença estatística significativa $(p>0,05)$. A média dos valores encontrados foi ligeiramente maior no período seco. Embora a chuva promova o deslocamento de detritos e materiais orgânicos das margens, o que pode aumentar a contaminação por coliformes; o aumento do fluxo, da vazão e do volume de água promove a diluição dos poluentes (Lemos et al., 2010; CETESB, 2012).

A Resolução CONAMA 357/2005 estabelece valores máximos permitidos para coliformes termotolerantes e admite que a E. coli seja determinada em substituição ao parâmetro, seguindo limites estabelecidos pelo órgão ambiental competente (CONAMA, 2005). Com exceção da E. coli, as bactérias que compõem o grupo dos coliformes termotolerantes não são de origem exclusivamente fecal, o que pode comprometer a detecção da poluição fecal. Dessa forma, a E. coli vem sendo cada vez mais utilizada no Brasil e no mundo como principal indicador de poluição fecal em ambientes aquáticos, já que é a única bactéria representante dos coliformes termotolerantes que tem origem exclusivamente fecal e está presente nas fezes humanas e de animais homeotérmicos (Hathaway et al., 2014).

Os resultados de E. coli obtidos foram comparados aos padrões estabelecidos pela CETESB na Decisão de Diretoria nº 027/2013/E de 09/04/2013 (CETESB, 2013). 
Tabela 2. Coliformes totais e E. coli no Córrego do Tanquinho, Ribeirão Preto, SP, Brasil, nos períodos seco e chuvoso - expressos em NMP $100 \mathrm{~mL}^{-1}$.

\begin{tabular}{|c|c|c|}
\hline \multicolumn{3}{|c|}{ Período Seco } \\
\hline Pontos de Coleta & Coliformes totais & Escherichia coli \\
\hline 1 & $2,30 \mathrm{E}+05$ & $7,80 \mathrm{E}+04$ \\
\hline 2 & $1,30 \mathrm{E}+07$ & $9,40 \mathrm{E}+05$ \\
\hline 3 & $4,90 \mathrm{E}+05$ & $2,30 \mathrm{E}+05$ \\
\hline 4 & $1,30 \mathrm{E}+07$ & $1,70 \mathrm{E}+06$ \\
\hline 5 & $4,90 \mathrm{E}+06$ & $1,70 \mathrm{E}+05$ \\
\hline Média & $6,32 \mathrm{E}+06$ & $6,24 \mathrm{E}+05$ \\
\hline DP & $6,37 \mathrm{E}+06$ & $6,92 \mathrm{E}+05$ \\
\hline Máximo & $1,30 \mathrm{E}+07$ & $1,70 \mathrm{E}+06$ \\
\hline Mínimo & $2,30 \mathrm{E}+05$ & $7,80 \mathrm{E}+04$ \\
\hline \multicolumn{3}{|c|}{ Período Chuvoso } \\
\hline 1 & $1,70 \mathrm{E}+06$ & $1,70 \mathrm{E}+05$ \\
\hline 2 & $1,30 \mathrm{E}+07$ & $1,30 \mathrm{E}+06$ \\
\hline 3 & $4,60 \mathrm{E}+06$ & $2,30 \mathrm{E}+05$ \\
\hline 4 & $7,90 \mathrm{E}+06$ & $3,40 \mathrm{E}+05$ \\
\hline 5 & $3,30 \mathrm{E}+06$ & $2,20 \mathrm{E}+05$ \\
\hline Média & $6,10 \mathrm{E}+06$ & $4,52 \mathrm{E}+05$ \\
\hline DP & $4,48 \mathrm{E}+06$ & $4,78 \mathrm{E}+05$ \\
\hline Máximo & $1,30 \mathrm{E}+07$ & $1,30 \mathrm{E}+06$ \\
\hline Mínimo & $1,70 \mathrm{E}+06$ & $1,70 \mathrm{E}+05$ \\
\hline VMP & - & 600 \\
\hline
\end{tabular}

VMP: Valor máximo permitido pela CETESB na Decisão de Diretoria $n^{\circ}$ 027/2013/E de 09/04/2013;

-: Não há VMP estabelecido.

Em todas as amostras analisadas, nos dois períodos, os valores de NMP $100 \mathrm{~m} \mathrm{~L}^{-1}$ ultrapassaram o limite de $600 \mathrm{NMP} 100 \mathrm{~m} \mathrm{~L}^{-1}$ estabelecido para rios de classe 2. O ponto 1 , local com menor quantidade de E. coli encontrada, apresentou NMP $100 \mathrm{~mL}^{-1}$ equivalente a $78.000(7,80 \mathrm{E}+04)$ no período seco e $170.000(1,7 \mathrm{E}+05)$ no período chuvoso (Tabela 2). No ponto 1 (local mais protegido em relação a todo leito do córrego), a detecção de E. coli tem grande importância sanitária, pois indica possível contaminação de origem animal silvestre e incremento de agentes patogênicos ao ser humano (WHO, 2011; Gasparotto, 2011). Por ser o ponto mais próximo à nascente, eram esperados valores menores para E. coli. Gasparotto (2011) realizou estudos em nascentes urbanas na cidade de Piracicaba, SP, Brasil, e encontrou 1600 NMP $100 \mathrm{~m} \mathrm{~L}^{-1}$ na nascente mais contaminada, valor muito inferior ao encontrado na nascente do Córrego do Tanquinho (78.000 NMP $100 \mathrm{~m} \mathrm{~L}^{-1}$ ). Pela sua localização no espaço urbano e industrial, o ponto 1 recebe resíduos e lixo provenientes das galerias pluviais, que escoam a água da chuva para a parte canalizada do córrego.

Os pontos 2 e 4 apresentaram os maiores valores de NMP $100 \mathrm{~m} \mathrm{~L}^{-1}$ para E. coli nos dois períodos (Tabela 2). Isto indica que, próximo a esses pontos, podem estar sendo lançadas 
elevadas cargas de esgoto doméstico, o que compatibiliza com a caracterização desses pontos e da bacia densamente urbanizados, como já mencionado.

O saneamento básico e a boa gestão da água são de grande importância para a saúde pública e, apesar dos avanços nas pesquisas, projetos e obras de saneamento, o número de mortes relacionadas a agentes patogênicos presentes na água ainda é elevado (Wilkes et al., 2011). A alta densidade de E. coli e coliformes totais encontrada no Córrego do Tanquinho pode indicar um alto grau de contaminação das águas, podendo transmitir doenças de veiculação hídrica como cólera, amebíase, giardíase, leptospirose, hepatite infecciosa, febre tifoide, paratifoide, diarreia aguda (Javed et al., 2014).

Os resultados das análises microbiológicas mostraram que as águas do Córrego do Tanquinho representam riscos para população. Além das enchentes que podem ocorrer no córrego (Tomazelli et al., 2010) e colocar a água em contato com a população, há relatos de pessoas, inclusive crianças, que fazem uso do córrego para pesca ou outros fins recreacionais. Essas práticas são comuns em córregos da cidade, e já foram observadas por Da Silva Alves et al. (2013) em estudo realizado no córrego Ribeirão Preto, principal curso de água superficial municipal.

Comparando os valores encontrados para E. coli nos períodos seco e chuvoso, não foi observada diferença estatística significativa $(p>0,05)$. Os resultados foram maiores no período chuvoso em três pontos (Tabela 2), porém a média dos valores encontrados foi maior no período seco devido principalmente ao alto valor encontrado no ponto 4. A lavagem do solo pelas chuvas poderia aumentar a contaminação por E. coli no córrego (O’Neill et al., 2013), já que as fezes de animais presentes nas margens podem ser deslocadas para o leito do rio. Entretanto, a provável elevada carga de esgoto recebida próximo ao ponto 4 , somada ao menor volume de água no período seco, o que resulta em menor diluição de poluentes, fizeram com que a média do de NMP $100 \mathrm{~mL}^{-1}$ fosse alta no período seco.

Como já discutido, foi observada correlação positiva entra os parâmetros microbiológicos e a Turbidez.

\subsection{Metais}

As concentrações dos metais analisados na água superficial do Córrego do Tanquinho, durante os períodos seco e chuvoso estão apresentadas na Tabela 3.

As concentrações de $\mathrm{Cu}$ estavam em desacordo com a legislação nos pontos 4 $\left(0,010 \mathrm{mg} \mathrm{L}^{-1}\right)$ e $5\left(0,011 \mathrm{mg} \mathrm{L}^{-1}\right)$ no período seco, e no ponto $4\left(0,015 \mathrm{mg} \mathrm{L}^{-1}\right)$ do período chuvoso. $\mathrm{O} \mathrm{Cu}$ é encontrado naturalmente em rochas, na água e no ar, e é um micronutriente importante para todos os organismos. Entretanto, em altas concentrações na água, o $\mathrm{Cu}$ pode se tornar tóxico, principalmente para plantas aquáticas (Nagajyoti et al., 2010). Além disso, o $\mathrm{Cu}$ pode ser lançado no ambiente por atividades antropogênicas e atingir os corpos hídricos por meio de resíduos industriais, como observado por Harguinteguy et al. (2014), que encontraram elevadas concentrações de $\mathrm{Cu}$ e outros metais no rio Suquía (Argentina) próximo a uma área de lançamento de efluentes industriais. Dessa forma, embora este metal seja encontrado naturalmente no ambiente e possa ser levado ao córrego por águas da chuva, altas concentrações de $\mathrm{Cu}$ observadas no ponto 4 , tanto no período seco como no chuvoso permitem supor o lançamento de resíduos industriais, haja visto a presença de industrias na bacia do córrego.

$\mathrm{O} \mathrm{Cu}$ e o $\mathrm{Zn}$ foram os únicos metais analisados no córrego que apresentaram correlação significativa $(\mathrm{p}<0,05)$ com $\mathrm{pH}$. Essa correlação foi negativa $(\mathrm{r}=-0,975)$ e observada apenas no período seco. $\mathrm{O}$ pH exerce grande influência na partição de metais entre as fases dissolvida e particulada. Nas águas naturais o $\mathrm{pH}$ é regido pelo equilíbrio carbonato-bicarbonato-dióxido de carbono e, quanto mais alto o valor de $\mathrm{pH}$, maior a quantidade de íons carbonato e bicarbonato. Os metais pesados são removidos da água (fase dissolvida) por precipitados de 
carbonato e bicarbonato. Logo, o aumento do $\mathrm{pH}$ pode provocar a diminuição da concentração de metais pesados na água (Duc et al., 2013).

Tabela 3. Concentração dos metais pesados $\left(\mathrm{mg} \mathrm{L}^{-1}\right)$ analisados na água superficial, do Córrego do Tanquinho, Ribeirão Preto, SP, Brasil, durante os períodos seco e chuvoso.

\begin{tabular}{ccccccc}
\hline \multicolumn{7}{c}{ Período Seco } \\
\hline Pontos de coleta & $\mathrm{Cu}$ & $\mathrm{Zn}$ & $\mathrm{Cr}$ & $\mathrm{Pb}$ & $\mathrm{Cd}$ & $\mathrm{Mn}$ \\
\hline Ponto 1 & 0,004 & 0,00032 & 0,0016 & 0,00053 & $\mathrm{ND}$ & 0,0014 \\
Ponto 2 & 0,0065 & 0,0028 & 0,0026 & 0,0024 & $\mathrm{ND}$ & 0,015 \\
Ponto 3 & 0,003 & 0,0026 & 0,0025 & 0,00035 & $\mathrm{ND}$ & 0,0014 \\
Ponto 4 & $0,010^{*}$ & 0,012 & 0,0021 & 0,00066 & 0,000013 & 0,0057 \\
Ponto 5 & $0,011^{*}$ & 0,014 & 0,0018 & 0,0026 & $\mathrm{ND}$ & 0,0071 \\
Média & 0,0069 & 0,0063 & 0,0021 & 0,0013 & 0,000013 & 0,00612 \\
\hline & & Período Chuvoso & & & \\
\hline Ponto 1 & 0,007 & 0,001 & 0,00084 & 0,0018 & $\mathrm{ND}$ & $0,46^{*}$ \\
Ponto 2 & 0,008 & 0,002 & 0,00091 & 0,00079 & $\mathrm{ND}$ & 0,054 \\
Ponto 3 & 0,009 & $\mathrm{~N} . \mathrm{D}$ & 0,00073 & 0,0017 & $\mathrm{ND}$ & 0,048 \\
Ponto 4 & $0,015^{*}$ & 0,001 & 0,00063 & 0,00057 & $\mathrm{ND}$ & 0,042 \\
Ponto 5 & 0,005 & 0,03 & 0,00054 & 0,0017 & 0,000065 & 0,032 \\
Média & 0,0088 & 0,0085 & 0,00073 & 0,0065 & 0,000065 & 0,1272 \\
VMP & 0,009 & 0,18 & 0,05 & 0,01 & 0,001 & 0,1 \\
\hline
\end{tabular}

VMP: Valor máximo permitido pela resolução CONAMA n $357 / 2005$ para águas classe 2;

ND: Não detectado;

*: Valor em desacordo com o VMP.

A concentração de Mn observada no ponto 1 no período chuvoso $\left(0,46 \mathrm{mg} \mathrm{L}^{-1}\right)$ é próxima à concentração máxima $\left(0,528 \mathrm{mg} \mathrm{L}^{-1}\right)$ encontrada por Jabeen et al. (2014) em água superficial próxima a um parque industrial na bacia Haripur do Paquistão. Valores similares já foram encontrados em análises realizadas no córrego Monte Alegre e em seus afluentes, também localizados no município de Ribeirão Preto. Nikaido et al. (2004) encontraram 0,49 mg.L $\mathrm{L}^{-1} \mathrm{de}$ Mn em sua concentração máxima no córrego Monte Alegre e Alves et al. (2010) obtiveram a concentração máxima de $0,229 \mathrm{mg} \mathrm{L}^{-1}$. A presença do $\mathrm{Mn}$ em águas superficiais pode ser devido a processos naturais como erosão de minerais e reações de oxirredução na interação entre sedimento e água que também podem liberar Mn na água (Abesser e Robinson, 2010). Foi observada diferença estatística significativa $(p<0,05)$ entre o período seco e chuvoso em relação às concentrações de $\mathrm{Mn}$, as quais foram maiores no período chuvoso. Isso pode ter ocorrido por alterações nas reações de oxirredução entre o sedimento depositado no fundo e a coluna d'água, já que as concentrações de Mn no sedimento se apresentaram de maneira inversa a da água. No período seco o Mn apresentou maior concentração no sedimento (Tabela 3) e menor concentração na água e no período chuvoso, o inverso. O Mn absorvido em altas 
concentrações pode ser tóxico e causar disfunções neurais e problemas no sistema nervoso central, como o Alzheimer e o Manganismo (Dieter et al., 2005).

A Resolução CONAMA n ${ }^{0}$ 344/2004 estabelece as diretrizes gerais e os procedimentos mínimos para avaliação do material a ser dragado em águas jurisdicionais brasileiras. Para a classificação do sedimento a resolução define critérios a partir de dois níveis. O nível 1 define os limiares abaixo dos quais prevê-se baixa probabilidade de efeitos adversos à biota e o nível 2 define o limiar acima do qual prevê-se um provável efeito adverso à biota (CONAMA, 2004).

As concentrações dos metais pesados analisados em sedimento no Córrego do Tanquinho, durante o período seco e chuvoso, estão apresentadas na Tabela 4.

Tabela 4. Concentração dos metais pesados $\left(\mathrm{mg} \mathrm{kg}^{-1}\right)$ analisados no sedimento do Córrego do Tanquinho, Ribeirão Preto, SP, Brasil, durante o período seco.

\begin{tabular}{lcccccc}
\hline \multicolumn{7}{c}{ Período Seco } \\
\hline Pontos de coleta & $\mathrm{Cu}$ & $\mathrm{Zn}$ & $\mathrm{Cr}$ & $\mathrm{Pb}$ & $\mathrm{Cd}$ & $\mathrm{Mn}$ \\
\hline Ponto 1 & 32,33 & 128,93 & 532,06 & 0,47 & 0,023 & 669,39 \\
Ponto 2 & 28,3 & 125,49 & 15,11 & 0,33 & 0,018 & 207,2 \\
Ponto 3 & 56,03 & 88,95 & 337,25 & 0,86 & 0,024 & 302,81 \\
Ponto 4 & 52,04 & 66,84 & 57,29 & 0,56 & 0,022 & 703,51 \\
Ponto 5 & 53,69 & 106,44 & 450,72 & 0,83 & 0,022 & 499,18 \\
Média & 44,48 & 103,33 & 278,49 & 0,61 & 0,02 & 476,42 \\
\hline & & & Período Chuvoso & & & \\
\hline Ponto 1 & 34,69 & 36,86 & 8,2 & 1,42 & 0,012 & 32,18 \\
Ponto 2 & 22,57 & 66,82 & 8,76 & 6,59 & 0,024 & 74,49 \\
Ponto 3 & 66,73 & 72,96 & 27,36 & 9,12 & 0,03 & 198,11 \\
Ponto 4 & 40,63 & 87,58 & 20,27 & 9,16 & 0,031 & 112,73 \\
Ponto 5 & 35,55 & 84,42 & 21,73 & 7,15 & 0,04 & 61,23 \\
Média & 40,04 & 69,73 & 17,26 & 6,69 & 0,03 & 95,75 \\
VMP Nível 1 & 35,7 & 123 & 37,3 & 35 & 0,6 & - \\
VMP Nível 2 & 197 & 315 & 90 & 91,3 & 3,5 & - \\
\hline
\end{tabular}

VMP: Valor máximo permitido pelo CONAMA 344/2004 para os níveis 1 e 2;

-: Não há VMP estabelecido.

Com exceção do $\mathrm{Cr}$, todos os metais analisados nos sedimentos apresentaram concentrações abaixo do limite estabelecido pela legislação para o nível 2. O Cr apresentou concentração superior a $90 \mathrm{mg} \mathrm{kg}^{-1}$ (nível 2) nos pontos 1,3 e 5 do período seco. Em contrapartida, no período chuvoso, o $\mathrm{Cr}$ não apresentou concentração superior ao limite do nível 2 e nem ao limite do nível 1, que é de $37,3 \mathrm{mg} \mathrm{kg}^{-1}$. Essa diferença na concentração de $\mathrm{Cr}$ entre os períodos foi estatisticamente significativa $(\mathrm{p}<0,05)$.

Altas concentrações de $\mathrm{Cr}$ e outros metais nos sedimentos de rios podem estar relacionadas à grande quantidade de minerais no sedimento, variando entre regiões com tipos de solos e minerais diferentes (Kelley e Hudson, 2007). Portanto, características minerais locais poderiam influenciar as altas concentrações de Cr nos sedimentos do Córrego do Tanquinho. Alves et al. (2010) também encontraram elevadas concentrações de Cr no córrego Monte Alegre do município de Ribeirão Preto. No entanto é importante salientar sobre a possibilidade de lançamento irregular de efluentes industriais, já que bairros industriais estão presentes na bacia 
do córrego, inclusive próximo ao ponto 1 , onde foi encontrada a maior concentração de Cr nos sedimentos. $\mathrm{O} \mathrm{Cr}$ pode estar presente em vários tipos de resíduos industriais oriundos da produção de têxteis, da fabricação de produtos químicos, da metalurgia básica, dentre outros (Jesus et al., 2004).

As concentrações de $\mathrm{Zn}$ também foram significativamente $(\mathrm{p}<0,05)$ maiores no período seco, com a maior concentração ocorrendo no ponto 1. Comparando os valores encontrados, o Zn apresentou-se acima do limite estabelecido para o nível 1 apenas nos pontos 1 e 2 do período seco. O menor fluxo e volume de água do período seco, principalmente no ponto 1 , facilitam a decantação de partículas em suspensão. Os metais que entram no ambiente aquático podem se ligar a essas partículas que decantam e se incorporar ao sedimento (Jesus et al., 2004). Por outro lado, na chuva, o maior fluxo de água e velocidade da correnteza podem revolver o sedimento e diminuir a concentração de metais no mesmo. Isso pode explicar as maiores concentrações de alguns metais no sedimento durante período seco, com destaque para o ponto 1 .

$\mathrm{O} \mathrm{Pb}$ foi único metal analisado em sedimento que apresentou maiores concentrações na chuva, com diferença estatística significativa $(\mathrm{p}<0,05)$. As concentrações de $\mathrm{Pb}$ de maneira geral foram baixas e não ultrapassaram os limites estabelecidos pela legislação para o nível 1.

Os metais podem atingir o ambiente aquático de forma descontínua, o que pode prejudicar a identificação da contaminação apenas em análises de água. O sedimento pode retratar condições históricas das influências antropogênicas sobre os corpos d'água, por isso vem sendo utilizado cada vez mais em avaliações ambientais (Roig et al., 2011).

\section{CONCLUSÃO}

Os resultados obtidos com o diagnóstico espacial e temporal do Córrego do Tanquinho mostraram que o corpo d'água recebe cargas poluidoras que afetam suas condições físico-químicas e microbiológicas.

Entre os parâmetros limnológicos analisados destaca-se o OD, com concentrações abaixo do limite estabelecido pela legislação.

As concentrações de coliformes totais e E. coli encontradas ao longo do córrego indicaram alta contaminação por esgotos domésticos. Grandes quantidades de E. coli pressupõe a presença de micro-organismos patogênicos que podem causar doenças de veiculação hídrica.

Entre os metais analisados na água superficial, apenas o $\mathrm{Cu}$ e o $\mathrm{Mn}$ apresentaram concentrações acima dos limites estabelecidos pela legislação. Nos sedimentos, destacou-se o Cr, em desacordo com a legislação em algumas amostras. Embora as altas concentrações de Cr e Mn possam estar relacionadas às características do solo da região, podem também indicar, juntamente com $\mathrm{Cu}$, possíveis lançamentos de efluentes industriais no córrego.

A influência temporal na qualidade da água não foi observada em todos os parâmetros analisados. Os parâmetros que apresentaram diferença estatística significativa $(p<0,05)$ entre os períodos seco e chuvoso foram o $\mathrm{pH}$, os metais $\mathrm{Mn}$ e $\mathrm{Cr}$ analisados em água superficial e os metais $\mathrm{Mn}, \mathrm{Cr}$ e $\mathrm{Pb}$ analisados em sedimentos do córrego.

O diagnóstico espacial destaca os pontos 2 e 4 com as piores condições microbiológicas, os pontos 4 e 5 com presença de alguns metais na água acima dos limites estabelecidos pela legislação.

É recomendada a realização de outros estudos que possam esclarecer melhor a origem das cargas poluidoras que afetam o córrego, e também, que o enquadramento do Córrego do Tanquinho seja revisto, buscando analisar se a classe 2 é a ideal para o contexto no qual está inserido esse corpo hídrico e tendo em vista também a sua importância para sociedade. Independente disso é imprescindível que as autoridades públicas conscientizem a população

Rev. Ambient. Água vol. 12 n. 2 Taubaté - Mar. / Apr. 2017 
sobre os riscos em utilizar as águas do córrego de maneira indevida, visto que, na atual situação, podem gerar graves problemas de saúde pública.

Os dados gerados com o presente trabalho podem contribuir com as autoridades públicas e órgãos gestores dos recursos hídricos não só com um possível reenquadramento do córrego e a conscientização da população, mas também em decisões que venham a ocorrer no sentido de recuperar e proteger o Córrego do Tanquinho.

\section{AGRADECIMENTOS}

Ao Conselho Nacional de Desenvolvimento Científico e Tecnológico (CNPq) pela concessão de bolsa de iniciação científica, e à Fundadação de Amparo à Pesquisa do Estado de São Paulo (FAPESP) pelo auxílio regular (Processo No 2013/07238-2).

\section{REFERÊNCIAS}

ABESSER, C.; ROBINSON, R. Mobilisation of iron and manganese from sediments of a Scottish upland reservoir. Journal of Limnology, v. 69, n. 1, p. 42-53, 2010. http://dx.doi.org/10.3274/JL10-69-1-04

ALVANI, J.; BOUSTANI, F.; TABIEE, O.; HASHEMI, M. The effects of human activity in Yasuj Area on the health of Stream city. International Journal of Social, Behavioral, Educational, Economic and Management Engineering, v. 50, p. 341-345, 2011.

ALVES R. I.; TONANI, K. A. A.; NIKAIDO, M.; CARDOSO, O. O.; TREVILATO, T. M. B.; SEGURA-MUÑOZ, S. I. Avaliação das concentrações de metais pesados em águas superficiais e sedimentos do Córrego Monte Alegre e afluentes, Ribeirão Preto, SP, Brasil. Ambiente e Água, v. 5, n. 3, p. 122-132, 2010. http://dx.doi.org/10.4136/ambiagua.157.

AMERICAN PUBLIC HEALTH ASSOCIATION; AMERICAN WATER WORKS ASSOCIATION; WATER ENVIRONMENT FEDERATION. Standard Methods for examination of water and wastewater. 22th. ed. Washington, 2012. $1360 \mathrm{p}$.

BAYRAM, A.; ÖNSOY, H.; BULUT, V. N.; AKINCI, G. Influences of urban wastewaters on the stream water quality: A case study from gumushane province, Turkey. Environmental Monitoring and Assessment, v. 185, n. 2, p. 1285-1303, 2013. http://dx.doi.org/10.1007/s10661-012-2632-y

COMPANHIA AMBIENTAL DO ESTADO DE SÃO PAULO. Águas superficiais: relatório de qualidade das águas superficiais do Estado de São Paulo. 2012. Disponível em http://www.cetesb.sp.gov.br/agua/aguas-superficiais/35-publicacoes-/-relatorios. Acesso em: 22 jul. 2015

COMPANHIA AMBIENTAL DO ESTADO DE SÃO PAULO. Decisão de Diretoria 112/2013/E, de 9 de abril de 2013. Dispõe sobre o estabelecimento dos valores limites do parâmetro Escherichia coli (E.coli), para avaliação da qualidade dos corpos de águas do território do Estado de São Paulo. Diário Oficial do Estado de São Paulo, São Paulo, SP, 12 abr. 2013.

COMPANHIA AMBIENTAL DO ESTADO DE SÃO PAULO. Guia nacional de coleta e preservação de amostras: água, sedimento, comunidades aquáticas e efluentes líquidos. São Paulo: CETESB; Brasília: ANA, 2011. 
CONSELHO NACIONAL DO MEIO AMBIENTE (Brasil). Resolução no 344 de 25 de março de 2004. Estabelece as diretrizes gerais e os procedimentos mínimos para a avaliação do material a ser dragado em águas jurisdicionais brasileiras, e dá outras providências. Diário Oficial [da] União, n. 87, 07 maio 2004, p. 56-57.

CONSELHO NACIONAL DO MEIO AMBIENTE (Brasil). Resolução n 357, de 17 de março de 2005. Dispõe sobre a classificação dos corpos de água e diretrizes ambientais para o seu enquadramento, bem como estabelece as condições e padrões de lançamento de efluentes, e dá outras providências. Diário Oficial [da] União, n. 53, 18 mar. 2005, p. 58-63.

DA SIlVA AlVES, R. I.; CARDOSO, O. O.; TONANI, K. A. A.; JUliÃO, F. C.; TREVILATO, T. M. B.; SEGURA-MUNÕZ, S.I. Water quality of the Ribeirão Preto Stream, a watercourse under anthropogenic influence in the southeast of Brazil. Environmental Monitoring and Assessment, v. 185, p. 1151-1161, 2013. http://dx.doi.org/10.1007/s10661-012-2622-0

DIETER, H. H.; BAYER, T. A.; MULTHAUP, G. Environmental Copper and Manganese in the Pathophysiology of Neurologic Diseases (Alzheimer's Disease and Manganism). Acta hydrochimica et hydrobiologica, v. 33, n. 1, p. 72-78, 2005. http://dx.doi.org/10.1002/aheh.200400556

DUC, T. A.; LOI, V. D.; THAO, T. T. Partition of heavy metals in a tropical river system impacted by municipal waste. Environmental Monitoring and Assessment, v. 185, n. 2, p. 1907-1925, 2013. http://dx.doi.org/10.1007/s10661-012-2676-z

GASPAROTTO, F. A. Avaliação ecotoxicológica e microbiológica da água de nascentes urbanas no município de Piracicaba - SP. 2011. 90 p. Dissertação (Mestrado em Ciências) - Centro de Energia Nuclear na Agricultura, Universidade de São Paulo, Piracicaba, 2011.

HARGUinteguY, C. A.; CIRELLI, A. F.; PIGNATA, M. L. Heavy metal accumulation in leaves of aquatic plant Stuckenia filiformis and its relationship with sediment and water in the Suquía river (Argentina). Microchemical Journal, v. 114, p. 111-118, 2014. http://dx.doi.org/10.1016/j.microc.2013.12.010

HATHAWAY, J. M.; KROMETIS, L. H.; HUNT, W. F. Exploring Seasonality in Escherichia coli and Fecal Coliform Ratios in Urban Watersheds. .Journal of Irrigation and Drainage Engineering, v. 140, n. 4, p. 04014003-1-04014003-6, 2014. http://dx.doi.org/10.1061/(ASCE)IR.1943-4774.0000700

JABEEN, S.; SHAH, M. T.; AHMED, I.; KHAN, S.; HAYAT, M. Q. Physico-chemical parameters of surface and ground water and their environmental impact assessment in the Haripur Basin, Pakistan. Journal of Geochemical Exploration, v. 138, p. 1-7, 2014. http://dx.doi.org/10.1016/j.gexplo.2013.12.004

JAVED, F.; AHMED, N. M.; SHAH, H. U.; IQBAL, M. S.; WAHID, A. ; AHMAD, S. S. Effects of Seasonal Variations on Physicochemical Properties and Concentrations of Faecal Coliform in River Kabul. World Applied Sciences Journal, v. 29, n. 1, p. $142-$ 149, 2014. http://dx.doi.org/10.5829/idosi.wasj.2014.29.01.2080 
JESUS, H. C. de; COSTA, E. de A.; MENDONÇA, A. S. F.; ZANDONADE, E. Distribution and abundance of heavy metals in sediments from Vitória Island estuarine system. Química Nova, v. 27, n. 3, p. 378-386, 2004. http://dx.doi.org/10.1590/S010040422004000300004

KELLEY, K. D.; HUDSON, T. Natural versus anthropogenic dispersion of metals to the environment in the Wulik River area, western Brooks Range, northern Alaska. Geochemistry: Exploration, Environment, Analysis, v. 7, n. 1, p. 87-96, 2007. http://dx.doi.org/10.1144/1467-7873/06-121

KRUPEK, R. A.; BRANCO, C. C. Z.; PERES, C. K. Variação sazonal de alguns parâmetros físicos e químicos em três rios pertencentes a uma bacia de drenagem na região centrosul do Estado do Paraná, Sul do Brasil. Acta Scientiarum Biological Sciences, v. 30, n. 4, p. 431-438, 2008. http://dx.doi.org/10.4025/actascibiolsci.v30i4.5873

LEMOS, M.; FERREIRA NETO, M.; DIAS, N. da S. Sazonalidade e variabilidade espacial da qualidade da água na Lagoa do Apodi, RN. Engenharia Agrícola e Ambiental, v. 14, n. 2, p. 155-164, 2010.

MEI, K.; LIAO, L.; ZHU, Y.; LU, P.; WANG, Z.; DAHLGREN, R. A.; ZHANG, M. Evaluation of spatial-temporal variations and trends in surface water quality across a rural-suburbanurban interface. Environmental Science and Pollution Research, v. 21, n. 13, p. 80368051, 2014. http://dx.doi.org/10.1007/s11356-014-2716-z

NAGAJYOTI, P. C.; LEE, K. D.; SREEKANTH, T. V. M. Heavy metals, occurrence and toxicity for plants: a review. Environmental Chemistry Letters, v. 8, n. 3, p. 199-216, 2010. http://dx.doi.org/10.1007/s10311-010-0297-8

NIKAIDO, M.; OLIVEIRA, A. DA S.; TREVILATO, T. M. B.; SEGURA-MUÑOZ, S. I. Análise da qualidade da água do córrego Monte Alegre e afluentes, Ribeirão Preto, SP, Brasil: enfoque para coliformes fecais e metais pesados. Mundo saúde, v. 28, n. 4, p. 414-420, 2004.

O’NEILL, S.; ADHIKARI, A. R.; GAUTAM, M. R.; ACHARYA, K. Bacterial contamination due to point and nonpoint source pollution in a rapidly growing urban center in an arid region. Urban Water Journal, v. 10, n. 6, p. 411-421, 2013.

PHILIPPI JR, A.; MARTINS G. Águas de Abastecimento. In: PHILIPPI JR., A. Saneamento, saúde e ambiente: fundamentos para um desenvolvimento sustentável. Barueri: Manole, 2005.

PURANDARA, B. K.; VARADARAJAN, N.; VENKATESH, B.; CHOUBEY,V. K. Surface water quality evaluation and modeling of Ghataprabha River, Karnataka, India. Environmental Monitoring and Assessment, v. 184, p. 1371-1378, 2012. http://dx.doi.org/10.1007/s10661-011-2047-1

RIBEIRÃO PRETO. Secretaria Municipal da Administração. Plano municipal de saneamento básico. 2012. Disponível em: http://www.ribeiraopreto.sp.gov.br/sadm/aud/pmsb_01_ 2012.pdf. Acesso em: 08 nov. 2015

ROIG, N.; NADAL, M.; SIERRA, J. et al. Novel approach for assessing heavy metal pollution and ecotoxicological status of rivers by means of passive sampling methods. Environment International, v. 37, n. 4, p. 671-677, 2011. http://dx.doi.org/10.1016/j.envint.2011.01.007 
SHIN, J. Y.; ARTIGAS, F.; HOBBLE, C.; LEE, Y. S. Assessment of anthropogenic influences on surface water quality in urban estuary, northern New Jersey: Multivariate approach. Environmental Monitoring and Assessment, v. 185, n. 3, p. 2777-2794, 2013. http://dx.doi.org/10.1007/s10661-012-2748-0

SIQUEIRA, G. W.; APRILE, F.; MIGUEIS, A. M. Diagnóstico da qualidade da água do rio Parauapebas (Pará - Brasil). Acta Amazonica, v. 42, n.3, p. 413-422, 2012.

SOUZA, C. L. de; ANDRADE, C. S.; SOUZA, C. L. de; ANDRADE, C. S. Health, environment and territory: a necessary discussion in health training. Ciência \& Saúde Coletiva, v. 19 , n. 10 , p. 4113-4122, 2014. http://dx.doi.org/10.1590/1413812320141910.08992014

TOMAZELLI, A. C.; LOPES, D. C.; FERRACINI JUNIOR, R. Avaliação da qualidade da água do córrego Tanquinho (Ribeirão Preto, SP, Brasil). Revista do Centro Universitário Barão de Mauá, v. 3, p. 32-42, 2010.

TONANI, K. A. A. Bioagentes patogênicos em águas residuárias: destaque para Giardia spp., Cryptosporidium spp., bactérias indicadoras e vírus entéricos. 2011.140 p. Tese (Doutorado em Ciências) - Escola de Enfermagem de Ribeirão Preto, Universidade de São Paulo, Ribeirão Preto, 2011.

WORLD HEALTH ORGANIZATION. Guidelines for drinking-water quality. 4th. ed. Genebra, 2011.

WILBERS, G. J.; BECKER, M.; NGA, L. T.; SEBESVARI, Z.; RENAUD, F. G. Spatial and temporal variability of surface water pollution in the Mekong Delta, Vietnam. Science of the Total Environment, v. 485-486, p. 653-665, 2014.

http://dx.doi.org/10.1016/j.scitotenv.2014.03.049

WILKES, G.; EDGE, T. A.; GANNON, V. P. J.; et al. Associations among pathogenic bacteria, parasites, and environmental and land use factors in multiple mixed-use watersheds. Water Research, v. 45, n. 18, p. 5807-5825, 2011. http://dx.doi.org/10.1016/j.watres.2011.06.021 


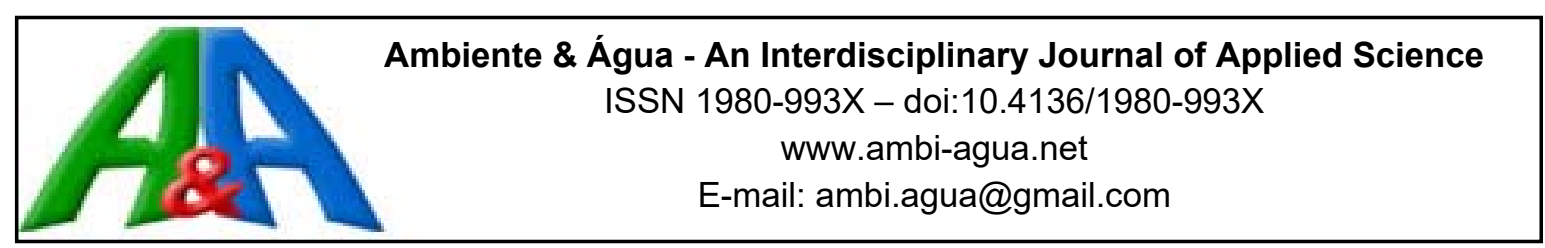

\title{
Gerenciamento de áreas contaminadas por postos de combustíveis em Cuiabá, Mato Grosso, Brasil
}

\author{
doi:10.4136/ambi-agua.1872 \\ Received: 01 Mar. 2016; Accepted: 30 Jan. 2017

\section{Suzy Darley de Lima; Andrea Ferreira de Oliveira; Rossean Golin; Danila Soares Caixeta; Zoraidy Marques de Lima; Eduardo Beraldo de Morais*} \\ Universidade Federal de Mato Grosso (UFMT), Cuiabá, MT, Brasil \\ Departamento de Engenharia Sanitária e Ambiental \\ *Autor correspondente: e-mail: beraldo_morais@yahoo.com.br, \\ suzyd.lima@gmail.com, oliveira27andrea@gmail.com, golin.rossean@gmail.com, \\ danilacaixeta@gmail.com, zoraidy@terra.com.br
}

\section{RESUMO}

A comercialização de combustíveis derivados de petróleo é uma atividade potencialmente poluidora. Tal impacto ocorre principalmente devido a acidentes no transporte com caminhão tanque e vazamentos nos tanques de armazenamento subterrâneo dos postos de combustíveis. O gerenciamento de áreas contaminadas tem o objetivo de reduzir os riscos à saúde humana por meio do conhecimento das características do local contaminado e dos impactos causados pelos contaminantes, proporcionando assim auxílio à tomada de decisão quanto às formas de intervenção mais adequadas. Neste trabalho, por meio da análise dos processos de licenciamento ambiental dos postos de combustíveis de Cuiabá, MT, Brasil, foi possível apresentar um panorama dos postos com algum tipo de contaminação ambiental, já que a investigação de passivos é obrigatória durante esse licenciamento. De 136 processos de licenciamento analisados, constatou-se que em 17 os laudos ambientais apresentaram algum tipo de contaminação no solo e/ou água subterrânea. Os principais contaminantes encontrados foram Benzeno, Tolueno, Etilbenzeno e Xilenos Totais (BTEX) e Hidrocarbonetos Policíclicos Aromáticos (HPA's). Em oito postos foram aplicadas as seguintes técnicas de remediação: três de atenuação natural, três de extração multifásica e três de bombeamento. Ressalta-se que nos nove postos restantes, não foi encontrada informação sobre a aplicação de técnicas de remediação. Destaca-se a necessidade do órgão ambiental estadual tornar pública as informações sobre as áreas contaminadas presentes não somente em Cuiabá, mas em todo o Estado. Segundo a Resolução CONAMA 420/2009 esta é uma obrigação do órgão ambiental para que o gerenciamento das áreas contaminadas possa ser efetivo. É importante também o Estado estabelecer políticas relativas à gestão das áreas contaminadas com legislações específicas ao tema, assim como alguns Estados já tem feito.

Palavras-chave: água subterrânea, hidrocarbonetos, licenciamento ambiental, passivo ambiental. 


\title{
Management of contaminated areas by gas stations in Cuiabá, Mato Grosso, Brazil
}

\begin{abstract}
The commercialization of petroleum-based fuels is a potentially polluting activity. Environmental impacts are mainly due to tanker truck accidents and leaking underground storage tanks of gas stations. The management of contaminated sites aims to reduce risks to human health and environment through measures that provide knowledge regarding the characteristics of contaminated site and the impacts of contaminants, thus allowing the most appropriate intervention to be determined. This paper analyzed the environmental licensing process of the Cuiaba-MT gas stations in order to present an overview of the gas stations responsible for some type of environmental contamination, since the investigation of liabilities is required for this license. One hundred and thirty-six licensing processes were analyzed, and it was found that 17 of these reported some type of contamination in soil and/or groundwater. The main contaminants were Benzene, Toluene, Ethylbenzene and Xylenes (BTEX) and Polycyclic Aromatic Hydrocarbons (PAHs). The following remediation techniques were applied: three cases of natural attenuation, three of multiphase extraction and three of pumping. This highlights the necessity for the State Environment Agency to make available information about contaminated areas not only in Cuiabá but throughout the State. According to CONAMA Resolution 420/2009, this is an obligation of the environmental agency for the management of contaminated areas to be effective. It is also important that the Mato Grosso State establish public policies for the management of contaminated areas, with legislation specific to the issue, as some other Brazilian states have done.
\end{abstract}

Keywords: environmental liability, environmental licensing, groundwater, hydrocarbons.

\section{INTRODUÇÃ̃O}

De acordo com a Agência Nacional de Petróleo (ANP), o Brasil tem apresentado acréscimo na instalação de postos revendedores de combustíveis automotivos, passando de 36.730 (2008) para 39.763 (2014) postos instalados, mantendo-se na quinta posição no ranking de maiores consumidores de petróleo (ANP, 2015).

Da mesma forma, verifica-se o aumento da quantidade de áreas contaminadas decorrentes desse tipo de atividade, como consta nos inventários efetuados pelos órgãos ambientais de alguns Estados brasileiros, como São Paulo e Minas Gerais, que possuem há mais tempo o gerenciamento de áreas contaminadas (Minas Gerais, 2015; CETESB, 2014). Este aumento está associado à publicação da Resolução CONAMA no 273/2000 (Brasil, 2000) que dentre as diretrizes instituídas, estabeleceu a obrigatoriedade da investigação de passivos ambientais para o licenciamento ambiental de postos de combustíveis e serviços.

Os passos para o gerenciamento ambiental de áreas contaminadas são descritos na Resolução CONAMA n ${ }^{\circ}$ 420/2009 (Brasil, 2009) que determina critérios e valores orientadores de referência de qualidade, de prevenção e de investigação do solo e valores de investigação para águas subterrâneas. A Resolução cita que na ocorrência comprovada de substâncias químicas que possam causar risco à saúde humana e ao meio ambiente, ações específicas para a proteção da população exposta devem ser desenvolvidas por órgãos competentes.

As áreas contaminadas têm sido uma constante preocupação para os gestores públicos, uma prova disso é o Estado de São Paulo, que foi pioneiro no levantamento e cadastramento dessas áreas. Desde 2002, a Companhia Ambiental do Estado de São Paulo (CETESB), órgão 
vinculado à Secretaria Estadual do Meio Ambiente, tem apresentado em forma de inventário uma lista com as áreas contaminadas, informando qual o setor que mais tem contribuído para contaminação do solo e das águas subterrâneas. Segundo esse relatório, a principal atividade responsável pelas áreas contaminadas no Estado são os postos de combustíveis com 3.825 registros (74\%), ficando bem a frente das demais atividades como: indústrias (17\%), comerciais (5\%), instalações para destinação de resíduos (3\%) e casos de acidentes, agricultura e fonte de contaminação de origem desconhecida (1\%) (CETESB, 2014).

Outros Estados também têm adotado essa ferramenta. Em 2007, Minas Gerais publicou a primeira lista de suas áreas contaminadas, que é atualizada anualmente pela Fundação Estadual do Meio Ambiente (FEAM). Atualmente, a cidade de Belo Horizonte possui 199 áreas contaminadas enquanto que nas demais regiões do Estado, 418 áreas possuem contaminação. As principais atividades econômicas associadas às áreas contaminadas são postos de combustíveis $(73 \%)$, indústria $(15 \%)$, ferrovias $(7 \%)$, mineradoras $(2 \%)$ e outras $(4 \%)$ (Minas Gerais, 2015).

O Estado do Rio de Janeiro após sancionar em dezembro de 2012 a Resolução CONEMA $n^{\circ} 44$ (Rio de Janeiro, 2012) que dispõe sobre a obrigatoriedade da identificação de eventual contaminação ambiental do solo e das águas subterrâneas, publicou uma lista e um mapa das áreas contaminadas e reabilitadas, por meio do Instituto Estadual do Ambiente (INEA). Ao todo são 328 áreas afetadas por diversos tipos de poluentes e os postos de combustíveis são os principais responsáveis por essas contaminações (58\%) (Rio de Janeiro, 2015).

O maior interesse em fazer um levantamento das áreas contaminadas por combustíveis derivados de petróleo surgiu devido à complexidade, toxicidade e mobilidade no ambiente dos compostos monoaromáticos do grupo BTEX (Benzeno, Tolueno, Etilbenzeno e Xileno Totais), presentes nos combustíveis. Segundo Andrade et al. (2010), compostos como os BTEX são tóxicos tanto ao meio ambiente como ao ser humano, em que atuam como depressores do sistema nervoso central e apresentam toxicidade crônica mais significativa que os hidrocarbonetos alifáticos (também presentes no petróleo e derivados), mesmo em concentrações da ordem de $\mu \mathrm{g} \mathrm{\textrm {L } ^ { - 1 }}$.

Uma vez constatada que a comercialização de combustíveis automotivos é a principal atividade responsável pela contaminação de solos e águas subterrâneas nos Estados citados, essa merece total atenção dos demais órgãos ambientais dos outros entes federativos. A Secretaria de Estado de Meio Ambiente de Mato Grosso (SEMA/MT) não possui um inventário das áreas contaminadas do Estado, ficando o alerta para a necessidade da elaboração do documento, pois por meio dele será possível a tomada de decisão mais eficaz para a minimização dos impactos. Segundo o último levantamento da ANP, o Estado de Mato Grosso tem 1.027 postos revendedores de combustíveis automotivos, ficando classificado como o $12^{\circ}$ Estado com maior quantidade de postos instalados no país (ANP, 2015).

O presente estudo tem como finalidade apresentar um panorama dos postos de combustíveis responsáveis por algum tipo de contaminação do solo e/ou da água subterrânea no município de Cuiabá, capital do Estado de Mato Grosso. O levantamento das áreas contaminadas foi feito por meio da análise documental dos processos de licenciamento ambiental dos postos de combustíveis existentes na Secretaria de Estado de Meio Ambiente (SEMA/MT). A necessidade do estudo realizado justifica-se pelo anseio de chamar a atenção para a importância de se inventariar essas áreas para que medidas adequadas possam ser tomadas. Além disso, é importante destacar que a atividade dos postos revendedores de combustíveis possui considerável potencial de contaminação das matrizes solo e água subterrânea, podendo restringir o uso de poços para abastecimento público instalados nas adjacências. 


\section{2. ÁREAS CONTAMINADAS}

Áreas contaminadas são descritas como locais com concentrações de poluentes representados por quaisquer substâncias ou resíduos que venham a causar danos ou riscos aos bens a proteger, como a qualidade das águas superficiais e subterrâneas, do solo e a saúde da população humana ou animal (CETESB, 2001). Geralmente, o surgimento de tais áreas está atrelado aos processos socioeconômicos ambientalmente não sustentáveis, que utilizam os recursos naturais sem observância aos parâmetros de proteção ambiental.

É importante ressaltar que áreas contaminadas e áreas degradadas não possuem o mesmo conceito. Áreas degradadas são locais que apresentam alterações negativas das suas propriedades físicas, tais como sua estrutura ou grau de compacidade, a perda de matéria devido à erosão e à alteração de características químicas, devido a processos como a salinização, lixiviação, deposição ácida e a introdução de poluentes. Áreas contaminadas são locais onde há comprovadamente poluição causada por quaisquer substâncias ou resíduos que tenham sido depositados, acumulados, armazenados, enterrados ou infiltrados, e que determina impactos negativos sobre os bens a proteger (CETESB, 2001). Sendo assim, as áreas contaminadas constituem um caso particular das áreas degradadas, e estão totalmente contidas nessas, ou seja, toda área contaminada é também uma área degradada (Günther, 2006).

Nos últimos anos, tem se observado uma ampla discussão entre os temas áreas contaminadas e áreas degradadas com o principal propósito de reintegrá-las à malha urbana. A necessidade de reutilização das áreas onde foram desenvolvidas atividades potencialmente poluidoras, como o caso de derramamento de combustíveis, tem se intensificado, sobretudo em regiões densamente povoadas e industrializadas. A sua reutilização de forma indiscriminada representa um grave aumento de risco à população como um todo e ao meio ambiente, pois podem funcionar como fontes dinâmicas de contaminação secundária, extrapolando os contaminantes para além da área afetada e para outros meios, estendendo seus efeitos deletérios (Günther, 2006).

O gerenciamento de áreas contaminadas visa minimizar os riscos que a população e o meio ambiente estão sujeitos, por meio de um conjunto de medidas que assegurem o conhecimento das características dessas áreas e dos impactos causados pelos contaminantes, proporcionando os instrumentos necessários à tomada de decisão quanto às formas de intervenção mais adequadas (CETESB, 2001). Os principais procedimentos e ações de investigação e de gestão durante esse processo devem seguir as seguintes etapas, segundo a Resolução CONAMA 420/2009 (Brasil, 2009): identificação, na qual uma avaliação preliminar e investigação confirmatória serão efetuadas; diagnóstico, que inclui a investigação detalhada e avaliação de risco com objetivo de subsidiar a etapa de intervenção; e intervenção, cuja finalidade é executar ações de controle para a eliminação do perigo ou redução a níveis toleráveis dos riscos, bem como o monitoramento da eficácia das ações executadas, considerando o uso atual e futuro da área. A Figura 1 apresenta detalhadamente estas etapas de gerenciamento de áreas contaminadas de acordo com o Anexo III da Resolução CONAMA 420/2009 (Brasil, 2009).

\subsection{Contaminação de solos e águas subterrâneas por derivados de petróleo}

Para se ter uma dimensão da problemática que envolve a contaminação do ambiente por hidrocarbonetos de petróleo, a Agência de Proteção Ambiental dos Estados Unidos (USEPA) estima que atualmente existam nesse país, aproximadamente, 571.000 tanques subterrâneos que armazenam derivados de petróleo e outras substâncias perigosas. Segundo o Programa de Tanques de Armazenagem Subterrâneos dessa agência, já foram registrados mais de 528.000 casos de vazamentos com consequente contaminação do solo e da água subterrânea sendo que 
aproximadamente 456.000 destas áreas contaminadas já foram recuperadas restando, portanto, 72.000 áreas em processo de remediação ou a ser remediada (USEPA, 2016).

Na Europa, a Agência Ambiental Europeia (European Environmental Agency - EEA) estima que aproximadamente 1.170 .000 áreas potencialmente contaminadas estão presentes em 27 países membros da EEA. A estimativa é que esse número representa $45 \%$ do total de áreas contaminadas presentes nos 39 países membros. O setor de serviços que mais contribui para essa contaminação são os postos de combustíveis com destaque principalmente em países como Holanda, Finlândia, Hungria, Croácia, Itália e Bélgica (EEA, 2014).

No Brasil, em meados de 1970, verificou-se um aumento nas instalações de postos de combustíveis. Nesta época os tanques de armazenamento de combustível eram construídos em chapa de aço simples, com uma vida útil de aproximadamente 20 a 25 anos. Após este período, muitos dos tanques não foram substituídos o que contribuiu para a ocorrência de vazamentos e contaminação ambiental (Oliveira e Loureiro, 1998).

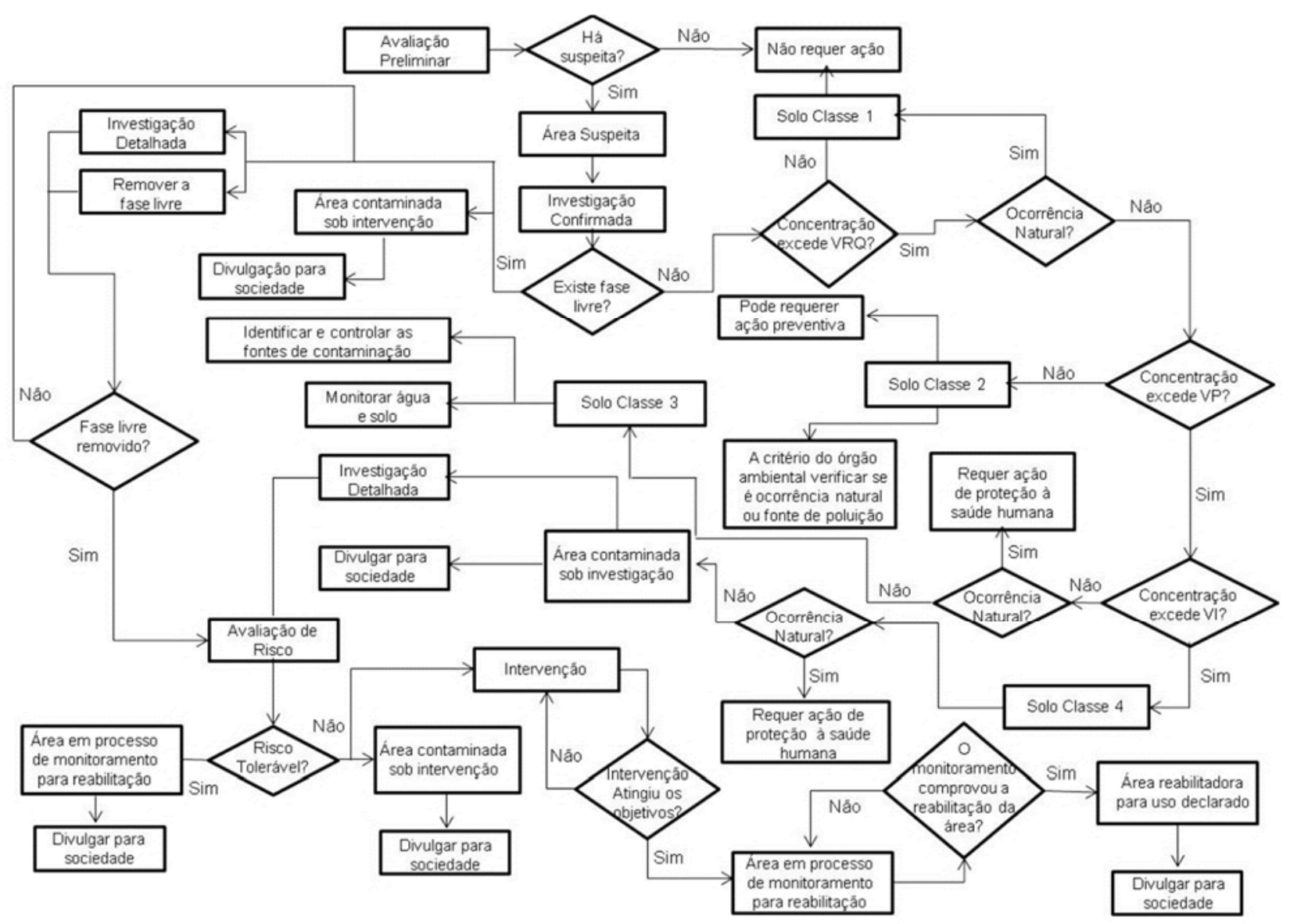

Figura 1. Fluxograma das etapas de gerenciamento de áreas contaminadas segundo a Resolução CONAMA 420/2009 (Brasil, 2009).

Quando há vazamentos de hidrocarbonetos em subsuperfície, estes podem se particionar em cinco fases distintas (CETESB, 2001):

1) Fase livre, quando existe produto puro em fase separada (imiscível ou parcialmente miscível) que apresenta mobilidade no meio poroso, podendo ser bombeado por fluir para o interior do poço; 
2) Fase residual, produto puro em fase separada (imiscível ou parcialmente miscível) que não apresenta mobilidade no meio poroso (não pode ser bombeado por não fluir para o interior do poço);

3) Fase vapor, quando o contaminante se encontra volatilizado, apresenta alta mobilidade, ocupando os espaços vazios existentes no solo, podendo acumular-se em espaços confinados como garagens subterrâneas, porões e utilidades (gás, telefonia, bueiros, entre outros);

4) Fase adsorvida, quando os contaminantes estão retidos nas partículas do solo por processos de adsorção, sobretudo em solos com alto teor de argila ou de matéria orgânica; e

5) Fase dissolvida, quando o contaminante se encontra dissolvido na água subterrânea, apresenta mobilidade muito elevada e é responsável pelo transporte do contaminante a grandes distâncias da fonte de contaminação.

O problema da contaminação de áreas adjacentes aos postos de combustíveis está relacionado com a difícil detecção do vazamento nos estágios iniciais. Diante dessa dificuldade, quando os efeitos da contaminação se tornam evidentes, o dano já pode ter atingido níveis alarmantes, assim, a saúde da população pode se encontrar em risco, e, em consequência disso, as ações remediadoras tornam-se urgentes (Silva, 2007).

Os compostos BTEX possuem alta solubilidade em água o que aumenta o risco de contaminação subterrânea (Anneser et al., 2008). A alta mobilidade desses compostos no sistema solo-água está relacionada com o baixo coeficiente de partição octanol-água, que conduz a uma baixa adsorção no solo favorecendo a contaminação das águas subterrâneas (Nakhla, 2003). Dessa forma, os compostos monoaromáticos do grupo BTEX encontrados nos combustíveis automotivos podem estar presentes dissolvidos na água subterrânea e podem restringir o uso de poços para abastecimento público instalados nas imediações de postos combustíveis que apresentam contaminações.

No Brasil, o etanol é adicionado à gasolina de acordo com a sua disponibilidade no mercado nacional. As diferentes formulações da gasolina/álcool podem influenciar o destino e transporte dos hidrocarbonetos, podendo assim, agravar a contaminação. Embora isso pudesse reduzir as emissões atmosféricas prejudiciais pelos automóveis, o problema da contaminação dos aquíferos é agravado, uma vez que o etanol pode exercer um efeito de cossolvente, aumentando a solubilidade do BTEX em água e, por conseguinte, resultando em concentrações mais elevadas e maiores prejuízos (Corseuil et al., 2011).

\subsection{Técnicas de remediação}

Uma vez constatada a contaminação de solos e águas, ações para a recuperação ambiental são necessárias para a proteção do ambiente e da saúde humana. Tal procedimento conhecido como remediação, implica em intervenção direta, com intuito de conter, isolar, remover ou reduzir as concentrações dos contaminantes presentes.

Diversas tecnologias para a remediação têm sido aplicadas em áreas contaminadas por derivados de petróleo. Dentre as que envolvem processos físicos e químicos podem ser citadas a lavagem de solo, extração de vapores, bombeamento e tratamento de águas subterrâneas, injeção de ar na zona saturada (air sparging), tratamento térmico, oxidação química, contenção e barreiras passivas e reativas. Também tem se destacado, devido ao menor custo e interferência mínima nas áreas contaminadas, as técnicas que removem o contaminante por meio de processos biológicos. Nesta categoria se destaca a atenuação natural, biorremediação e fitorremediação (Khan et al., 2004). A Tabela 1 apresenta breve descrição dessas principais técnicas de remediação. 
Tabela 1. Principais técnicas de remediação de áreas contaminadas.

Técnica Descrição

Lavagem de solo

Utiliza líquidos, geralmente água e solvente, e processos mecânicos para extrair os contaminantes do solo. Os solventes são escolhidos baseados na sua capacidade de solubilizar os contaminantes e não possuir ou ter baixa toxicidade.

Extração de vapores

É uma tecnologia que promove a remoção de compostos orgânicos voláteis (VOCs) da zona não saturada do solo por meio de aplicação de vácuo em poços próximos à fonte de contaminação.

Injeção de ar na zona

Aplicado na remediação de VOCs dissolvidos na água subterrânea e saturada (air sparging) adsorvidos na zona saturada do solo por meio da injeção de ar atmosférico sob pressão. Assim, ocorre a volatilização dos contaminantes em fase adsorvida e/ou biodegradação aeróbica.

Bombeamento e tratamento de águas subterrâneas

Por meio de poços de extração, as águas subterrâneas contaminadas são removidas e tratadas por diversas tecnologias. Uma vez remediadas são reintroduzidas no aquífero ou descartadas em corpos hídricos superficiais.

Tratamento térmico

Envolve o aquecimento do solo a temperaturas que variam de 100 a $600{ }^{\circ} \mathrm{C}$ com o objetivo de promover a separação dos contaminantes orgânicos pela volatilização ou destruição.

Oxidação química

Tratamento que utiliza oxidantes químicos como ozônio, peróxidos e persulfatos, para decompor, reduzir ou eliminar a toxicidade dos contaminantes em solos e águas subterrâneas.

Contenção

Consiste basicamente em criar barreiras físicas ou hidráulicas para impedir a dispersão dos contaminantes para áreas adjacentes.

Consistem na passagem da água subterrânea contaminada através de uma barreira permeável instalada em subsuperfície transversalmente ao sentido

Barreiras passivas e reativas de escoamento, interceptando a pluma de contaminação. Três tipos de barreiras são utilizadas: de adsorção, na qual os contaminantes ficam adsorvidos e são removidos; de precipitação, os contaminantes dissolvidos na água são precipitados e removidos; reativa, que promove a quebra dos compostos tóxicos em produtos inertes.

Atenuação natural controlada

Conhecida também como remediação intrínseca ou passiva, usa processos naturais que ocorrem na área contaminada com o objetivo de reduzir as concentrações dos contaminantes, toxicidade, massa e/ou volume até níveis adequados à proteção da saúde humana e ao meio ambiente.

Biorremediação

Por meio do metabolismo de microrganismos (bactérias e fungos), nativos ou exóticos à área contaminada, os contaminantes são degradados em compostos com menor ou sem toxicidade.

Fitorremediação

Plantas são utilizadas para remediar áreas contaminadas por meio da degradação, extração, contenção ou imobilização dos contaminantes orgânicos e inorgânicos do solo e água subterrânea.

Fonte: CETESB (2001) e Khan et al. (2004). 


\section{MATERIAL E MÉTODOS}

Neste trabalho, foram analisados os processos de licenciamento ambiental dos postos de combustíveis do município de Cuiabá, capital do Estado de Mato Grosso. Esta análise foi iniciada em março de 2014, com uma consulta ao banco de dados da SEMA/MT, denominado Sistema Integrado de Monitoramento e Licenciamento Ambiental (SIMLAM Público), e nesta etapa foi necessário o auxílio de um técnico da empresa responsável pela elaboração e manutenção do sistema, pois nem todas as informações estavam dispostas no site da SEMA/MT. O término das análises ocorreu em outubro de 2014.

A partir da avaliação do SIMLAM Público foram identificados 190 processos de licenciamento ambiental referentes a postos de combustíveis e em seguida uma análise documental desses processos foi efetuada, com auxílio dos técnicos da Coordenadoria de Serviços e do Setor de Arquivo da SEMA/MT. Dos 190 processos constantes do banco de dados digital, 136 foram encontrados no arquivo físico e analisados.

Assim, foi possível identificar os postos que já tiveram algum tipo de passivo ambiental oriundos de vazamentos em alguma parte do sistema de armazenamento subterrâneo ou até mesmo provenientes de derramamento de combustíveis no momento do abastecimento dos tanques e abastecimento dos veículos automotivos, tendo como consequência a contaminação do solo e/ou da água subterrânea. As informações extraídas da avaliação dos processos de licenciamento se basearam nas informações contidas nos relatórios de áreas contaminadas publicados pelos Estados de São Paulo e Minas Gerais, que já se encontram disponibilizados ao público de maneira geral. Dessa forma, foram pesquisadas as informações: postos de combustíveis com contaminação de hidrocarbonetos no solo e/ou na água subterrânea, fonte de contaminação, meios impactados, tipo de contaminantes presentes, presença de fase livre e técnica de remediação aplicada para eliminação, redução ou contenção da pluma de contaminação.

Um mapa com a localização dos postos de combustíveis no município de Cuiabá que apresentaram algum tipo de contaminação foi confeccionado utilizando o software ArcGis 10.2 com a base de dados geográficos da SEMA/MT, no qual foram adicionadas as coordenadas dos postos de combustíveis constantes nos processos analisados.

\section{RESULTADOS}

Após a análise de 136 processos de licenciamento ambiental de postos de combustíveis da cidade de Cuiabá, MT, Brasil, foram constatados que em 17 (12,6\%) processos, os laudos ambientais apresentaram concentrações de hidrocarbonetos que representam algum tipo de contaminação nas matrizes solo e/ou água subterrânea. Dezesseis desses postos com contaminação estão localizados na área urbana da capital, local com grande densidade domiciliar (Figura 2).

Os laudos ambientais também demonstraram que a água subterrânea foi atingida pela pluma de contaminação em $13(76 \%)$ postos de combustíveis e em três foram constatados a presença de fase livre (Tabela 2). As investigações das contaminações ocorreram entre os anos de 2005 a 2014, e os principais compostos avaliados foram os BTEX, hidrocarbonetos policíclicos aromáticos (HPA's) e compostos orgânicos voláteis (VOC).

Em quatro postos de combustíveis $(6,7,13$ e 15) foram detectadas emanações gasosas de compostos orgânicos voláteis (VOC) no solo, que variaram de 999 a 4.620 ppm (Tabela 2), porém, esses locais não foram submetidos a uma investigação detalhada para saber se 0 contaminante estava presente apenas na fase de vapor. Sabendo-se que um vazamento de derivados de petróleo geralmente se distribui em diversas fases (vapor, livre, residual, adsorvida 
e dissolvida), é necessário fazer análises nas matrizes solo e água subterrânea para saber a real distribuição dos contaminantes no ambiente.

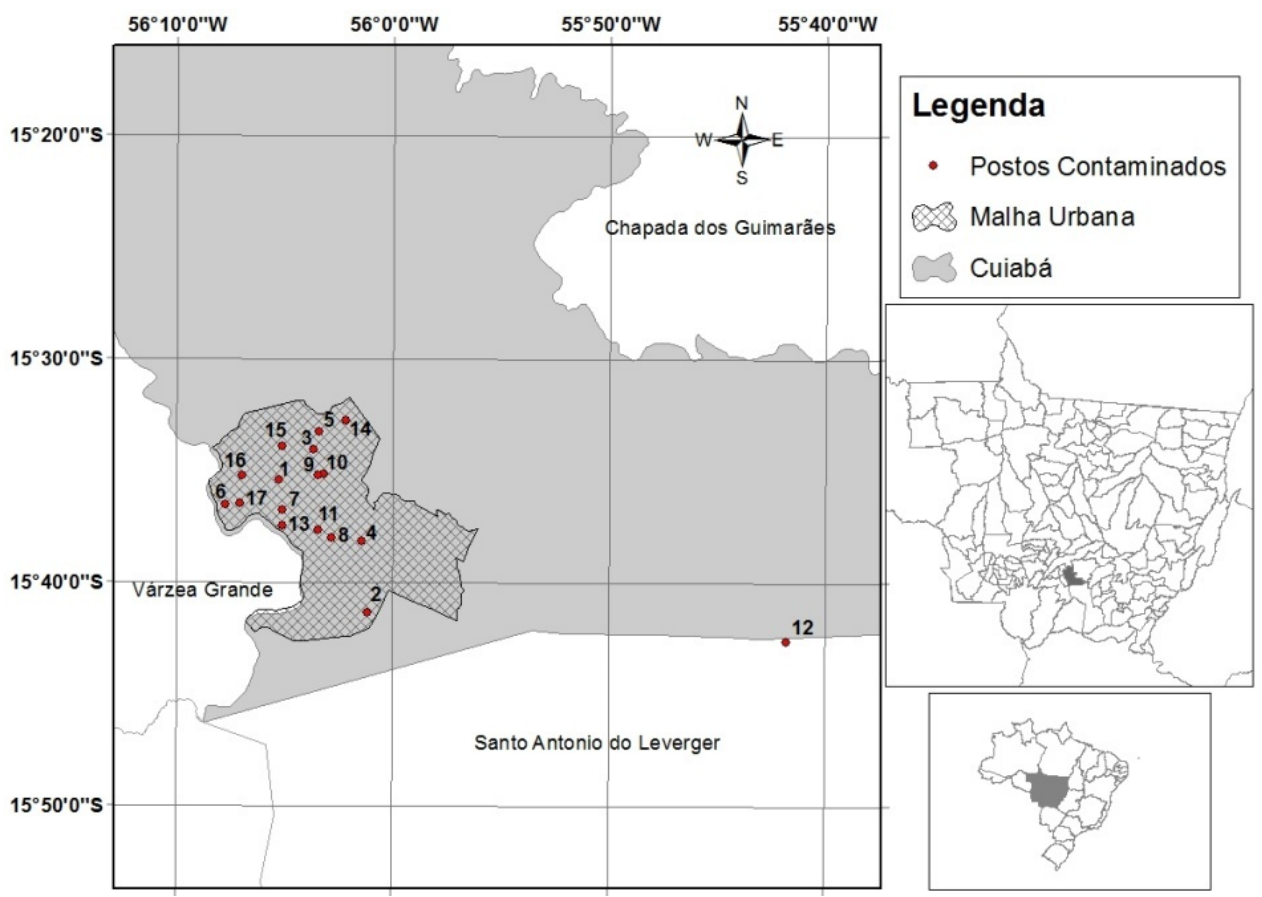

Figura 2. Localização dos postos de combustíveis com contaminação em Cuiabá, MT, Brasil.

Fonte: Organização dos autores.

Tabela 2. Características das contaminações dos postos de combustíveis no município de Cuiabá, MT, Brasil.

\begin{tabular}{cllcl}
\hline $\begin{array}{c}\text { Posto de } \\
\text { combustível }\end{array}$ & $\begin{array}{c}\text { Ano da } \\
\text { investigação }\end{array}$ & \multicolumn{1}{c}{ Meios impactados } & Fase livre & VOC (ppm) \\
\hline 1 & 2011 & Água Subterrânea e Solo & Não & $>10.000$ \\
2 & 2006 e 2011 & Água Subterrânea e Solo & Sim & $2006-$ até $7.280 /$ \\
3 & 2007 e 2010 & Água Subterrânea e Solo & Não & até 1.660 \\
4 & 2008 & Água Subterrânea e Solo & Não & até 11.000 \\
5 & 2006 e 2009 & Água Subterrânea e Solo & Sim $^{1}$ & até 11.000 \\
6 & 2011 & Solo & - & até 4.620 \\
7 & 2005 & Solo & - & até 4.400 \\
8 & 2009 & Água Subterrânea e Solo & Não & nd \\
9 & 2005 e 2006 & Água Subterrânea e Solo & Não & até 11.000 \\
10 & 2009 & Água Subterrânea & Não & nd \\
11 & 2009 e 2011 & Água Subterrânea & Sim & nd \\
12 & 2014 & Água Subterrânea & Não & nd \\
13 & 2006 & Solo & - & até 2.200 \\
14 & 2013 & Água Subterrânea & Não & nd \\
15 & 2009 e 2013 & Solo & - & até 999 \\
16 & 2013 & Água Subterrânea e Solo & Não & até 327 \\
17 & 2014 & Água Subterrânea e Solo & Não & até 360 \\
\hline
\end{tabular}

${ }^{1}$ Removida; nd $=$ não detectado. 
Os valores de BTEX e HPA's encontrados nos solos e águas subterrâneas adjacentes aos postos de combustíveis são apresentados nas Tabelas 3 e 4 . Destaca-se que em alguns processos de licenciamento (postos de combustíveis 2, 3 e 4) os valores individuais dos contaminantes não foram apresentados e sim o somatório de BTEX e HPA's (Tabela 5). Essa forma de apresentação dos resultados não é adequada, pois impede a comparação dos valores encontrados com aqueles orientadores estabelecidos pela CONAMA 420/2009 (Brasil, 2009).

Verificou-se ainda que nos processos de licenciamento ambiental dos postos 16 e 17 constam as informações da presença de contaminação e que análises dos passivos ambientais foram efetuadas em 2013 e 2014 respectivamente, porém não há documentos anexados referentes às quantidades de BTEX e HPA's encontrados nas matrizes solo e água subterrânea. Da mesma forma, para os postos 9 e 11 não foram apresentados os laudos da determinação desses poluentes nos processos, sendo encontrado somente a afirmação da contaminação.

Tabela 3. Concentração de BTEX e HPA's em solos adjacentes aos postos de combustíveis. As concentrações acima dos valores orientadores para solos residenciais estão destacadas em negrito.

\begin{tabular}{lccccccc}
\hline Posto de Combustível & 1 & 5 & 8 & 10 & 12 & 14 & $\begin{array}{c}\text { Valores } \\
\text { orientadores }\end{array}$ \\
\hline Benzeno & \multicolumn{7}{c}{ BTEXs $\left(\mathrm{mg} \mathrm{kg}^{-1}\right)$} \\
Etilbenzeno & nd & $\mathbf{8 , 2 3 9}$ & $\mathbf{0 , 1 0 2}$ & nd & nd & nd & 0,08 \\
Tolueno & 1,044 & 7,472 & 1,380 & nd & 0,0096 & nd & 40 \\
Xilenos & 0,021 & 13,572 & 0,501 & nd & nd & $<0,001$ & 30 \\
\hline & 6,58 & 24,5 & 11,854 & nd & nd & nd & 30 \\
\hline Acenafleno & 0,02 & 0,1186 & nd & nd & 0,028 & nd & $*$ \\
Acenaftileno & nd & 0,0777 & nd & nd & nd & nd & $*$ \\
Antraceno & nd & 0,0208 & nd & nd & $<0,010$ & nd & $*$ \\
Benzo(a)antraceno & nd & 0,0095 & nd & nd & nd & nd & 20 \\
Benzo(b)fluoranteno & nd & 0,0041 & nd & nd & nd & nd & $*$ \\
Benzo(k)fluoranteno & nd & 0,0018 & nd & nd & nd & nd & $*$ \\
Benzo(g,h,i)perileno & nd & nd & nd & nd & nd & nd & $*$ \\
Benzo(a)pireno & nd & 0,001 & nd & nd & nd & nd & 1,5 \\
Criseno & nd & 0,0457 & nd & nd & $<0,010$ & nd & $*$ \\
Dibenzo(a,h)antraceno & nd & nd & nd & nd & 0,070 & nd & 0,6 \\
Fenantreno & $<0,01$ & 1,153 & nd & nd & 0,151 & nd & 40 \\
Fluoranteno & nd & 0,0324 & nd & nd & nd & nd & $*$ \\
Fluoreno & $<0,01$ & 0,2597 & nd & nd & 0,062 & nd & $*$ \\
Indeno (1,2,3-c,d)pireno & nd & nd & nd & nd & nd & nd & 25 \\
Naftaleno & 2,56 & 14,384 & 0,1402 & nd & 0,070 & nd & 60 \\
Pireno & nd & 0,0619 & nd & nd & 0,014 & nd & $*$ \\
\hline Reslucac & & & & & \\
\hline
\end{tabular}

${ }^{1}$ Resolução CONAMA 420/2009 (Brasil, 2009).

nd $=$ não detectado.

*Valores não conformes aos estabelecidos pela Resolução CONAMA 420/2009 (Brasil, 2009). 
Tabela 4. Concentração de BTEX e HPA's em águas subterrâneas adjacentes aos postos de combustíveis. As concentrações acima dos valores orientadores estão destacadas em negrito.

\begin{tabular}{|c|c|c|c|c|c|c|c|}
\hline Posto de Combustível & 1 & 5 & 8 & 10 & 12 & 14 & $\begin{array}{c}\text { Valores } \\
\text { orientadores }^{1}\end{array}$ \\
\hline \multicolumn{8}{|c|}{ BTEXs $\left(\mu \mathrm{g} \mathrm{L}^{-1}\right)$} \\
\hline Benzeno & nd & 13246,5 & 1971,37 & 125,39 & 325,02 & 28,4 & 5 \\
\hline Etilbenzeno & 3112,9 & 1174,1 & 866,12 & 98,15 & 91,37 & nd & 300 \\
\hline Tolueno & 363,5 & 3902,5 & 3158,80 & 87,34 & nd & nd & 700 \\
\hline Xilenos & 13345 & 4562,9 & 5718,80 & 409,83 & 413,19 & nd & 500 \\
\hline \multicolumn{8}{|c|}{ HPA's $\left(\mu \mathrm{g} \mathrm{L}^{-1}\right)$} \\
\hline Acenafleno & $<1,5$ & 1,146 & 5,885 & 15,502 & 49,760 & nd & $*$ \\
\hline Acenaftileno & $<1,5$ & 0,337 & nd & 2,637 & nd & nd & $*$ \\
\hline Antraceno & $<1,5$ & 0,048 & nd & nd & 10,442 & nd & $*$ \\
\hline Benzo(a)antraceno & nd & nd & nd & nd & 4,005 & nd & 1,75 \\
\hline Benzo(b)fluoranteno & nd & nd & nd & nd & $<1,000$ & nd & $*$ \\
\hline Benzo(k)fluoranteno & nd & nd & nd & nd & $<1,000$ & nd & $*$ \\
\hline Benzo(g,h,i)perileno & nd & nd & nd & nd & $<1,000$ & nd & $*$ \\
\hline Benzo(a)pireno & nd & nd & nd & nd & $<1,000$ & nd & 0,7 \\
\hline Criseno & nd & 0,045 & nd & nd & 15,241 & nd & $*$ \\
\hline Dibenzo $(a, h)$ antraceno & nd & nd & nd & nd & nd & nd & 0,18 \\
\hline Fenantreno & $<1,5$ & 2,627 & nd & 7,357 & 180,642 & nd & 140 \\
\hline Fluoranteno & nd & 0,053 & 3,767 & 5,657 & 1,638 & nd & * \\
\hline Fluoreno & $<1,5$ & 1,656 & 4,348 & 11,379 & 79,084 & nd & $*$ \\
\hline $\begin{array}{l}\text { Indeno }(1,2,3-\mathrm{c}, \mathrm{d}) \\
\text { pireno }\end{array}$ & nd & nd & nd & nd & nd & nd & 0,17 \\
\hline Naftaleno & 106,95 & 344,967 & 430,639 & 204,961 & 294,798 & nd & 140 \\
\hline Pireno & nd & 0,101 & nd & nd & 18,207 & nd & $*$ \\
\hline
\end{tabular}

${ }^{1}$ Resolução CONAMA 420/2009 (Brasil, 2009).

nd $=$ não detectado.

*Valores não conformes aos estabelecidos pela Resolução CONAMA 420/2009 (Brasil, 2009).

Tabela 5. Somatório das concentrações de BTEX e HPA's encontrados nos solos e águas subterrâneas adjacentes aos postos de combustíveis 2,3 e 4.

\begin{tabular}{cccccc}
\hline \multirow{2}{*}{$\begin{array}{c}\text { Posto de } \\
\text { combustível }\end{array}$} & \multicolumn{2}{c}{ Água subterrânea $\left(\mu \mathrm{g} \mathrm{L}^{-1}\right)$} & & \multicolumn{2}{c}{ Solo $\left(\mathrm{mg} \mathrm{Kg}^{-1}\right)$} \\
\cline { 2 - 3 } \cline { 5 - 6 } & $\Sigma$ BTEX & $\Sigma$ HPA & & $\Sigma$ BTEX & $\Sigma$ HPA \\
\hline 2 & 81,9 & 6,15 & & nd & nd \\
3 & $997,0^{*} 996,282^{* *}$ & $90,856^{*} 13,320^{* *}$ & & $0,047^{*}$ & $0,3762^{*}$ \\
4 & 2727,8 & 386,872 & & 0,057 & 0,3620 \\
\hline
\end{tabular}

*ano de 2007; ** ano de 2009.

nd = não detectado. 
Outra informação importante para o gerenciamento das áreas contaminadas é em relação ao tipo de remediação que tem sido aplicada nessas áreas, sendo que em três casos a remediação tem ocorrido de forma natural e em nove casos não consta nos processos de licenciamento ambiental se algum tipo de remediação foi aplicado (Tabela 6). Vale destacar que a remediação natural é baseada na capacidade de atenuação natural dos contaminantes no solo e água subterrânea, e para ser considerada uma técnica efetiva de remediação é necessário um estudo criterioso da evolução da pluma de contaminação, uma metodologia de avaliação de risco e monitoramento durante todo o período necessário para que se atinjam as metas de remediação desejáveis (CETESB, 2001).

A técnica de bombeamento foi aplicada em três casos (Tabela 6). Esta técnica consiste em remover as águas contaminadas, por meio de poços de extração, para que ela seja tratada e reintroduzida no aquífero ou descartada em um corpo hídrico. Segundo São Paulo (CETESB, 2001), este é um dos métodos mais antigos de remediação e embora recentemente tenha sido substituído ou utilizado em combinação com outros métodos de remediação, ainda é largamente usado para remediar águas subterrâneas contaminadas em todo o mundo.

A técnica de extração multifásica (ventilação a vácuo) foi aplicada em três casos (Tabela 6). O sistema combina as técnicas de bioventilação e remoção de massa a vácuo, possibilitando a extração da fase livre, fase vapor, fase dissolvida na matriz do solo e estimulando o processo de biodegradação natural na zona não saturada (Khan et al., 2004).

Tabela 6. Técnicas de remediação aplicadas nas áreas contaminadas pelos postos de combustíveis em Cuiabá, MT, Brasil.

\begin{tabular}{|c|c|}
\hline Posto de Combustível & Técnica de Remediação \\
\hline 1 & Atenuação Natural \\
\hline 2 & Bombeamento \\
\hline 4 & $\begin{array}{l}\text { Bombeamento emergencial (água) e } \\
\text { Extração Multifásica (solo) }\end{array}$ \\
\hline 5 & Mini - Extração Multifásica \\
\hline 9 & $\begin{array}{l}\text { Extração Multifásica - sistema de } \\
\text { ventilação a vácuo }\end{array}$ \\
\hline 10 & Atenuação Natural \\
\hline 11 & Bombeamento \\
\hline 14 & Atenuação Natural \\
\hline $\begin{array}{l}3,6,7,8,12,13,15 \\
16 \text { e } 17\end{array}$ & $\begin{array}{l}\text { Nenhuma informação encontrada sobre a } \\
\text { aplicação de técnicas de remediação }\end{array}$ \\
\hline
\end{tabular}

\section{DISCUSSÃO}

Os principais poluentes orgânicos liberados durante um vazamento em tanques de armazenamento subterrâneo em postos de combustíveis são representados principalmente por diesel e gasolina. Tais combustíveis são constituídos principalmente por HPA's, BTEX e hidrocarbonetos alifáticos. O benzeno é o composto mais tóxico dentre os BTEX devido a suas características tóxicas e carcinogênicas mesmo em concentrações da ordem de

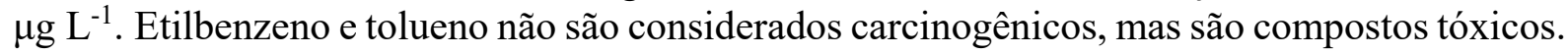
Xilenos são misturas de três isômeros (orto, meta e para-xileno) e possuem dois grupos metil 
ligados ao anel benzênico. Apesar de serem menos perigos que os outros BTEX eles podem causar doenças do fígado, rins e sistema nervoso (Rosales et al., 2014).

De acordo com a Portaria do Ministério da Saúde n 2.914/2011 (Brasil, 2011), o benzeno possui padrão de potabilidade de $5 \mu \mathrm{g} \mathrm{L}^{-1}$. Já os demais compostos, tolueno, etilbenzeno e xilenos são regulados com concentração máxima permitida em águas subterrâneas, de 170, 200 e $300 \mu \mathrm{g} \mathrm{L} \mathrm{L}^{-1}$, respectivamente, quando o uso preponderante é o consumo humano, segundo a Resolução CONAMA 396/2008 (Brasil, 2008).

Alguns passivos ambientais nos postos de combustíveis apresentaram valores de BTEX em água subterrânea acima do preconizado por essas legislações (Tabela 4). Valores acima dos valores orientadores estabelecidos pela Resolução CONAMA 420/2009 (Brasil, 2009) também foram encontrados principalmente para águas subterrâneas (Tabelas 3 e 4). Este fator passa a ser relevante para a distribuição das ameaças ao meio ambiente, pois a contaminação pode atingir um elevado número de pessoas quando esta atinge as águas subterrâneas. Tal fato pode se tornar ainda mais crítico em Cuiabá devido às condições de intermitência no abastecimento de água em muitos bairros, principalmente aqueles localizados na periferia, o que levam os moradores a construir poços rasos para a captação de água do nível freático, ou seja, água que está mais sujeita a contaminação.

A constatação de que técnicas de remediação não foram aplicadas em $53 \%$ dos postos que apresentaram contaminação pode ser considerada alarmante. No gerenciamento de áreas contaminadas, a remediação é uma das ações que possibilitam a mitigação dos impactos ambientais para a contínua ocupação e reocupação dessas áreas por meio da minimização e controle dos riscos, sendo uma etapa obrigatória segundo a Resolução CONAMA 420/2009 (Brasil, 2009). E mesmo nos casos em que as técnicas remediadoras foram aplicadas nas áreas contaminadas, informações referentes ao monitoramento posterior à remediação não foram encontradas nos processos. Entretanto, segundo os técnicos da Coordenadoria de Serviços e do Setor de Arquivo da SEMA/MT, documentos com estas informações podem não ter sido anexados aos mesmos.

A seleção e implementação de técnicas de remediação de áreas contaminadas no Brasil ainda é uma atividade que necessita de desenvolvimento na maioria dos estados. Segundo Cunha (2014), apesar do grande número de empresas de consultoria na área de investigação e remediação de áreas contaminadas, o país ainda carece de profissionais com capacitação técnica e científica o que acaba levando a ações de investigações mal planejadas e executadas e ineficiência dos sistemas de remediação. Prova disso é o fato da técnica de bombeamento e tratamento estar relacionada como a mais utilizada segundo dados disponibilizados pela Cetesb (2014) no Estado de São Paulo, ainda que sua ineficiência para promover a remediação de áreas contaminadas tenha sido relatada pelo National Research Council, nos EUA, na década de 1990 (Cunha, 2014).

Conforme relatado anteriormente, a SEMA/MT não possui uma publicação com as informações das áreas contaminadas do Estado de Mato Grosso, incluindo as áreas contaminadas por hidrocarbonetos de petróleo, o que pode dificultar o eficiente gerenciamento dessas áreas. Vale lembrar que a Resolução CONAMA 420/2009 (2009) no seu artigo 38, aponta que os órgãos ambientais competentes, deverão dar publicidade às informações sobre áreas contaminadas identificadas e suas principais características, na forma de um relatório.

Alguns estados brasileiros têm avançado no sentido de estabelecer legislações específicas para regulamentar essa matéria. Orientações das ações dos responsáveis pela contaminação assim como a atuação de profissionais técnicos tem sido encontrada na Lei Estadual $n^{\circ}$ 13.577/2009 em São Paulo (São Paulo, 2009), na Deliberação Normativa Conjunta COPAM/CERH n ${ }^{\circ}$ 02/2010 em Minas Gerais (Minas Gerais, 2010), na Portaria FEPAM ${ }^{\circ}$ 49/2014 no Rio Grande do Sul (Rio Grande do Sul, 2014) e na Resolução CONEMA nº 44/2012 no Rio de Janeiro (Rio de Janeiro, 2012). Além disso, também é importante a definição de 
padrões e valores orientadores levando em consideração as particularidades de cada Estado para que o gerenciamento de áreas contaminadas possa ser desenvolvido de forma adequada.

Este trabalho abordou apenas as áreas que foram contaminadas por derivados de petróleo em postos de combustíveis no município de Cuiabá, o que aponta a necessidade de avaliar os outros 140 municípios do Estado. Entretanto, não somente a atividade comercializadora de combustíveis automotivos deve ser considerada, pois Mato Grosso é um Estado com destaque para a agropecuária e mineração, atividades que também possuem potencial de contaminar o meio ambiente. Alguns trabalhos, inclusive, já demonstraram a contaminação de águas superficiais e subterrâneas do Estado por pesticidas (Cassara et al., 2012; Nogueira et al., 2012; Ribeiro et al., 2013), mercúrio (Vieira e Alho, 2004) e cromo (Cunha e Shiraiwa, 2011) destacando a necessidade de ampliar as investigações das áreas contaminadas.

\section{CONCLUSÕES}

O gerenciamento de áreas contaminadas configura um grande desafio ambiental para órgãos reguladores, empreendedores e sociedade em geral, considerando que o uso dessas áreas pode conferir riscos à saúde humana. Neste trabalho, um panorama dos postos de combustíveis responsáveis por algum tipo de contaminação ambiental em Cuiabá, MT, Brasil, foi apresentado. A identificação das contaminações foi efetuada por meio da análise dos processos de licenciamento ambiental dos postos de combustíveis que se mostrou uma ferramenta importante, já que a investigação de passivos ambientais é uma das etapas do processo do licenciamento. Foram identificados 17 postos com algum tipo de contaminação no solo e/ou água subterrânea, sendo os contaminantes BTEX, hidrocarbonetos policíclicos aromáticos e compostos orgânicos voláteis os principais poluentes encontrados nesses locais, como era de se esperar devido à natureza da atividade desenvolvida. Alguns passivos ambientais nos postos de combustíveis apresentaram valores de BTEX nas águas subterrâneas acima do preconizado pelas legislações brasileiras. O número de postos com passivo ambiental pode ser maior que os 17 encontrados, pois 54 processos de licenciamento não foram encontrados nos arquivos da Secretaria de Estado de Meio Ambiente (SEMA/MT). Em apenas nove processos foram encontradas informações sobre a aplicação de técnicas de remediação sendo elas: bombeamento, extração multifásica e atenuação natural. A SEMA/MT não apresenta uma compilação das informações dessas áreas contaminadas na forma de um relatório conforme indicado na Resolução CONAMA 420/2009, o que poderia facilitar o gerenciamento das mesmas. O desenvolvimento de políticas pelo Estado de Mato Grosso relativas à gestão das áreas contaminadas com legislações específicas ao tema se mostra necessário e irá nortear o efetivo gerenciamento das áreas contaminadas.

\section{AGRADECIMENTOS}

À Secretaria de Estado de Meio Ambiente (SEMA/MT), pela atenção e disposição dos dados referentes a essa pesquisa. À CAPES pela concessão de bolsa de estudos de mestrado.

\section{REFERÊNCIAS}

ANDRADE, J. A.; AUGUSTO, F.; JARDIM I. C. S. F. Biorremediação de solos contaminados por petróleo e seus derivados. Eclética Química, v. 35, n. 3, p. 17-43, 2010. http://dx.doi.org/10.1590/S0100-46702010000300002

Rev. Ambient. Água vol. 12 n. 2 Taubaté - Mar. / Apr. 2017 
ANNESER, B.; EINSIEDL, F.; MECKENSTOCK, R. U.; RICHTERS, L.; WISOTZKY, F.; GRIEBLER, C. High-resolution monitoring of biogeochemical gradients in a tar oilcontaminated aquifer. Applied Geochemistry, v. 23, n. 6, p. 1715-1730, 2008. http://dx.doi.org/10.1016/j.apgeochem.2008.02.003

AGÊNCIA NACIONAL DE PETRÓLEO, GÁS NATURAL E BIOCOMBUSTÍVEIS - ANP (Brasil). Anuário estatístico brasileiro do petróleo, gás natural e biocombustíveis 2015. Rio de Janeiro, 2015.

BRASIL. Ministério do Meio Ambiente. Conselho Nacional do Meio Ambiente. Resolução no 273, de 29 de novembro de 2000. Brasília, 2000.

BRASIL. Ministério do Meio Ambiente. Conselho Nacional do Meio Ambiente. Resolução no 396, de 03 de abril de 2008. Brasília, 2008.

BRASIL. Ministério do Meio Ambiente. Conselho Nacional do Meio Ambiente. Resolução no 420, de 28 de dezembro de 2009. Brasília, 2009.

BRASIL. Ministério da Saúde. Portaria no 2.914 de 12 de dezembro de 2011. Brasília, 2011.

CASARA, K. P.; VECCHIATO, A. B.; LOURENCETTI, C.; PINTO, A. A.; DORES, E. F. G. C. Environmental dynamics of pesticides in the drainage area of the S??o Louren??o River headwaters, Mato Grosso State, Brazil. Journal of the Brazilian Chemical Society. v.23, n.9, p.1719-1731, 2012. http://dx.doi.org/10.1590/S0103-50532012005000037

COMPANHIA AMBIENTAL DO ESTADO DE SÃO PAULO - CETESB. Relação de áreas contaminadas e reabilitadas no Estado de São Paulo. São Paulo, 2014, 14 p.

COMPANHIA AMBIENTAL DO ESTADO DE SÃO PAULO - CETESB. Manual de gerenciamento de áreas contaminadas. 2001. Disponível em: http://areascontaminadas.cetesb.sp.gov.br/manual-de-gerenciamento/. Acesso em: 02 maio 2016

CORSEUIL, H. X.; MONIER, A. L.; FERNANDES, M.; SCHNEIDER, M. R.; NUNES, C.C.; DO ROSARIO, M. et al. BTEX plume dynamics following an ethanol blend release: geochemical footprint and thermodynamic constraints on natural attenuation. Environmental Science and Technology, v. 45, n. 8, p. 3422-3429, 2011. http://dx.doi.org/10.1021/es104055q

CUNHA, R. C. A. Prefácio. In: MORAES, S. L.; TEIXEIRA, C. E.; MAXIMIANO, A. M. S. (Org.). Guia de elaboração de planos de intervenção para o gerenciamento de áreas contaminadas. São Paulo: IPT, 2014. p. 5-7.

CUNHA, L. F. J.; SHIRAIWA, S. Aplicação do método eletromagnético indutivo na investigação da pluma de contaminação da água subterrânea por resíduos de cromo de curtume. Revista Brasileira de Geofísica, v.29, n.1, p.127-134, 2011. http://dx.doi.org/10.1590/S0102-261X2011000100009

EUROPEAN ENVIRONMENT AGENCY - EEA. Progress in the management of Contaminated Sites in Europe. Ispra: Institute for Environment and Sustainability, 2014, $72 \mathrm{p}$.

GÜNTHER, W. M. R Áreas contaminadas no contexto da gestão urbana. São Paulo em Perspectiva, v. 20, n. 2, p. 105-117, 2006. 
KHAN, F. I.; HUSAIN, T.; HEJAZI, R. An overview and analysis of site remediation technologies. Journal of Environmental Management, v. 71, n. 2, p. 95-122, 2004. http://dx.doi.org/10.1016/j.jenvman.2004.02.003

MINAS GERAIS. Deliberação Normativa Conjunta COPAM/CERH nº 02, de 08 de setembro de 2010. Institui o Programa Estadual de Gestão de Áreas Contaminadas, que estabelece as diretrizes e procedimentos para a proteção da qualidade do solo e gerenciamento ambiental de áreas contaminadas por substâncias químicas. Diário do Executivo - Minas Gerais, Belo Horizonte, 2010.

MINAS GERAIS. Fundação Estadual do Meio Ambiente. Inventário de áreas contaminadas do Estado de Minas Gerais - 2015. Belo Horizonte, 2015. 45 p.

NAKHLA, G. Biokinetic modeling of in situ bioremediation of BTX compounds - impact of process variable and scaleup implications. Water Research, v. 37, n. 6, p. 1296-1307, 2003. http://dx.doi.org/10.1016/S0043-1354(02)00491-8

NOGUEIRA, E. N.; DORES, E. F. G. C., PINTO, A. A.; AMORIM, R. S. S.; RIBEIRO, M. L.; LOURENCETTI, C. Currently used pesticides in water matrices in central-western Brazil. Journal of the Brazilian Chemical Society. v. 23, n. 8, p. 1476-1487, 2012. http://dx.doi.org/10.1590/S0103-50532012005000008

OLIVEIRA, L. I.; LOUREIRO, C. O. Contaminação de aquíferos por combustíveis orgânicos em Belo Horizonte: avaliação preliminar. In: CONGRESSO BRASILEIRO DE ÁGUAS SUBTERRÂNEAS, 10., 1998, São Paulo. Anais... São Paulo: Associação Brasileira de Águas Subterrâneas, 1998.

RIBEIRO, A. C. A.; DORES, E. F. G. C.; AMORIM, R. S. S.; LOURENCETTI, C. Resíduos de pesticidas em águas superficiais de área de nascente do rio São Lourenço-MT: validação de método por extração em fase sólida e cromatografia líquida. Química Nova. v. 36, n. 2, p. 284-290, 2013. http://dx.doi.org/10.1590/S0100-40422013000200015

RIO DE JANEIRO. Resolução CONEMA no 44, de 14 de dezembro de 2012. Diário Oficial do Estado do Rio de Janeiro, Rio de Janeiro, 2012.

RIO DE JANEIRO. Instituto Estadual do Ambiente. Gerenciamento de áreas contaminadas do Estado do Rio de Janeiro. 3. ed. Rio de Janeiro, 2015.

RIO GRANDE DO SUL. Fundação Estadual de Proteção Ambiental Henrique Luiz Roessler. Portaria FEPAM no 49 de 28 de maio de 2014. Porto Alegre: Secretaria do Meio Ambiente, 2014.

ROSALES, R. M.; MARTÍNEZ-PAGÁN, P.; FAZ, A.; BECH, J. Study of subsoil in former petrol stations in SE of Spain: Physicochemical characterization and hydrocarbon contamination assessment. Journal of Geochemical Exploration, v. 147, p. 306-320, 2014. http://dx.doi.org/10.1016/j.gexplo.2014.10.006

SÃO PAULO. Lei $\mathrm{n}^{\circ} 13.577$, de 8 de julho de 2009. Dispõe sobre diretrizes e procedimentos para a proteção da qualidade do solo e gerenciamento de áreas contaminadas, e dá outras providências correlatas. Diário Oficial do Estado, São Paulo, Seção I, 9 jul. 2009. p.1-3 
SILVA, F. G. Gestão de áreas contaminadas e conflitos ambientais: o caso da cidade dos Meninos. 2007. 110 f. Dissertação (Mestrado em Ciências em Planejamento Energético) - Instituto Alberto Luiz Coimbra de Pós-Graduação e Pesquisa de Engenharia, Universidade Federal do Rio de Janeiro, Rio de Janeiro, 2007.

UNITED STATES. Environmental Protection Agency - USEPA. Cleaning Up Underground Storage Tank (UST) Releases. Disponível em: https://goo.gl/WBj6DY. Acesso em: 16 maio 2016.

VIEIRA, L. M.; ALHO, C. J. R. Contaminação por mercúrio em sedimento e moluscos da bacia do rio Bento Gomes, MT. Corumbá: Embrapa Pantanal, 2004. 20p. 


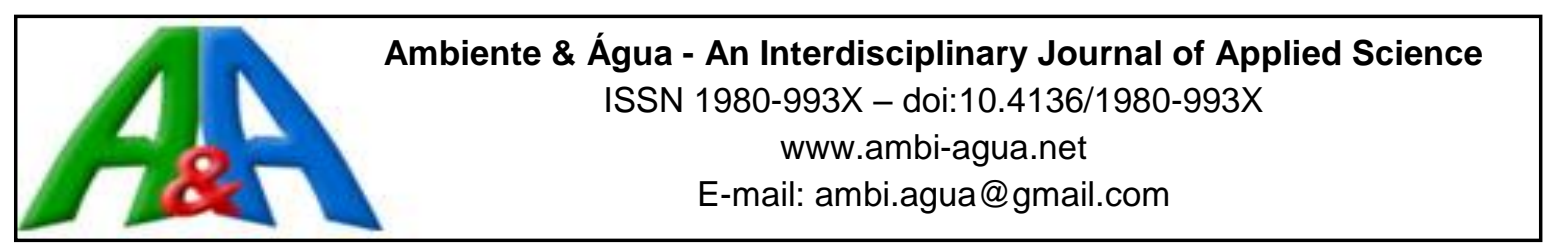

\title{
Secas e seus impactos no município de Boqueirão, PB, Brasil
}

\author{
doi:10.4136/ambi-agua.2004
}

Received: 13 Sep. 2016; Accepted: 18 Jan. 2017

\author{
André Aires de Farias*; Francisco de Assis Salviano de Sousa; \\ João Miguel Moraes Neto; Anailson de Sousa Alves \\ Universidade Federal de Campina Grande (UFCG), Campina Grande, PB, Brasil \\ Centro de Tecnologia e Recursos Naturais (CTRN) \\ *Autor correspondente: e-mail: andreaires61@ @otmail.com, \\ fsouza2011@gmail.com,moraes@deag.ufcg.edu.br, anailson_agro@hotmail.com
}

\section{RESUMO}

Secas são fenômenos naturais que ocorrem quando a precipitação é inferior às médias pluviométricas de uma determinada região. Contudo, não há um consenso para se definir o real significado do tema, em razão de que as variáveis hidrometeorológicas e socioeconômicas são diferentes nas diversas regiões do mundo. Objetivou-se caracterizar e analisar as secas e seus impactos no município de Boqueirão - PB. Para se identificar as secas foi utilizado o Índice Padronizado de Precipitação (IPP) com dados de 1963-2014. Com relação aos impactos, foram analisados dados referentes à agricultura (de 2011, 2012 e 2013), dados da pecuária e de produtos agropecuários produzidos nos anos de 2011, 2012, 2013 e 2014. As secas mais graves no município de Boqueirão ocorreram nos anos de 1980-1982, 1998-2000 e 2012-2013. Destas, a mais impactante foi a de 1998-2000. Essa seca reduziu drasticamente a produção agropecuária e o abastecimento de água da região. Os impactos mais intensos durante a seca 2012-2014 ocorreram em 2012 e 2013. As culturas agrícolas temporárias foram mais impactadas do que as permanentes. Isso ocorreu porque a maioria das permanentes eram irrigadas, diferente das temporárias. A maior redução de animais também ocorreu durante 2012 e 2013, com uma relação direta entre precipitação e o efetivo dos rebanhos. Não houve redução do valor da produção em função das secas.

Palavras-chave: déficit hídrico, índices de seca, risco climático.

\section{Droughts and their impacts on the municipality of Boqueirão, PB, Brazil}

\begin{abstract}
Droughts are natural phenomena that occur when precipitation falls below average in a given region. However, no consensus exists regarding the definition of this term due to differing hydro meteorological and socioeconomic variables in various regions of the world. In this work, we identified and analyzed droughts and their impacts on the municipality of Boqueirão - PB. We used the Standardized Precipitation Index (SPI) to identify droughts. Regarding impacts, we analyzed agricultural data from 2011, 2012 and 2013 as well as data relating to livestock and agricultural products produced in 2011, 2012, 2013 and 2014. The most severe droughts in Boqueirão municipality occurred in the years 1980-1982, 1998-2000 and 2012-2013. The greatest impact was observed in 1998-2000. The droughts have drastically reduced agriculture
\end{abstract}


production and water supply in the region. The most severe impacts during the drought of 20122014 occurred in 2012 and 2013. Temporary crops were more affected than the permanent ones. This was because most of the permanent crops were irrigated, unlike the temporary ones. The greatest reduction of animals also occurred during 2012 and 2013, with a direct relationship between precipitation and herd populations. The droughts caused no reduction in the value of production.

Keywords: climate risk, drought indices, water deficit.

\section{INTRODUÇÃO}

A seca é um fenômeno natural presente em todas as regiões do mundo. De todos os desastres naturais ocorridos, a seca é responsável por $22 \%$ dos gastos, $33 \%$ do número de pessoas afetadas e 3\% do número de mortos. A seca é o mais complexo de todos os fenômenos naturais, afetando um maior número de pessoas do que qualquer outro (Wilhite et al., 2007). Diferenças nas variáveis hidrometeorológicas e em fatores socioeconômicos, bem como a natureza estocástica da demanda de água em diferentes regiões ao redor do mundo, tornaramse um obstáculo para ter uma definição precisa de seca. Mesmo assim, a Organização Meteorológica Mundial (WMO, 1986) define seca como uma deficiência na precipitação. Já a Convenção das Nações Unidas de Combate à Desertificação e à Seca (UNCCD, 1994) define como um fenômeno que ocorre naturalmente quando a precipitação foi significativamente abaixo dos níveis normais registrados, provocando um sério desequilíbrio hídrico que afeta negativamente o meio urbano e rural.

A seca é uma condição natural que causa uma ampla gama de impactos sociais, econômicos e ambientais, desde a redução da produção agrícola até restrições na utilização de água nas residências. Secas de longa duração ou eventos de secas severas frequentes em regiões áridas e semiáridas podem levar a consequências ainda mais devastadoras, como a redução do abastecimento de alimentos e a desertificação (Mishra e Singh, 2010). Sistemas de monitoramento capazes de detectar e mapear secas em grandes escalas espaciais e com continuidade temporal são essenciais para avaliar a gravidade e extensão da seca e atenuar os seus impactos (Li e Rodell, 2015).

As características da precipitação são componentes muito utilizados para a redução dos riscos climáticos (Bordi et al., 2009). Nos últimos anos, o Índice padronizado de precipitação (IPP) (McKee et al., 1993) tem sido amplamente utilizado e se destacado sobre os outros índices (Guttman, 1999) e (Keyantash e Dracup, 2002). Assim, o IPP foi aceito pela WMO como o índice mais efetivo para o monitoramento de secas e redução de riscos (Hayes et al., 2011).

Além disso, o IPP foi projetado para quantificar o déficit ou excesso de precipitação em múltiplas escalas de tempo. As mais curtas são usadas para caracterizar as secas meteorológicas, estas são importantes para as atividades agrícolas, pois a umidade do solo tem uma resposta rápida em relação às anomalias de precipitação. Escalas de tempo mais longas são usadas para monitorar, principalmente, as secas hidrológicas, pois estas são de grande importância no abastecimento das fontes superficiais e subterrâneas (Sirdas e Sen, 2003).

Vários estudos analisaram a evolução espaço-temporal do IPP em diferentes escalas de tempo e em diversas regiões, entre eles Lloyd-Huges e Saunders (2002), Sonmez et al. (2005), Vicente-Serrano (2006), Livada e Assimakopoulos (2007) e Zhai et al. (2010). Outras pesquisas identificaram tendências de períodos secos e chuvosos nas séries temporais analisadas ao redor do mundo, Bordi et al. (2009), Santos et al. (2010) e Fischer et al. (2013).

No Brasil, Teixeira et al. (2013) observaram que dos 1164 meses analisados em Bagé, RS, Brasil, quanto aos valores de IPP, 6,8\% foram classificados como eventos de seca severa e extrema. Farias et al. (2014) em pesquisa no município de Taperoá-PB, observaram que houve 
uma grande variabilidade temporal das séries pluviométricas analisadas, o município apresentou meses extremamente secos seguidos de meses úmidos ou normais. Os IPPs detectaram, segundo a intensidade média, secas severas e extremas. Embora não esteja no artigo, a categoria de seca moderada aconteceu com maior frequência.

Data de 1583 o primeiro relato de uma seca no Nordeste do Brasil (NEB), feito pelo Padre Fernão Cardin, (BRASIL, 1981), porém é consenso que o fenômeno seca é tão antigo quanto o surgimento do ser humano na Terra. Então, os seguintes questionamentos são levantados para o município de Boqueirão-PB: Em que anos ocorreram secas mais graves na série 1963-2014? Quais foram os impactos da seca de 2012-2014? Diante do que foi apresentado, objetivou-se identificar e analisar as secas e seus impactos no município de Boqueirão, PB, Brasil.

\section{MATERIAL E MÉTODOS}

A área de estudo compreende o município de Boqueirão-PB, localizado na Mesorregião da Borborema e na Microrregião do Cariri Oriental (Figura 1).

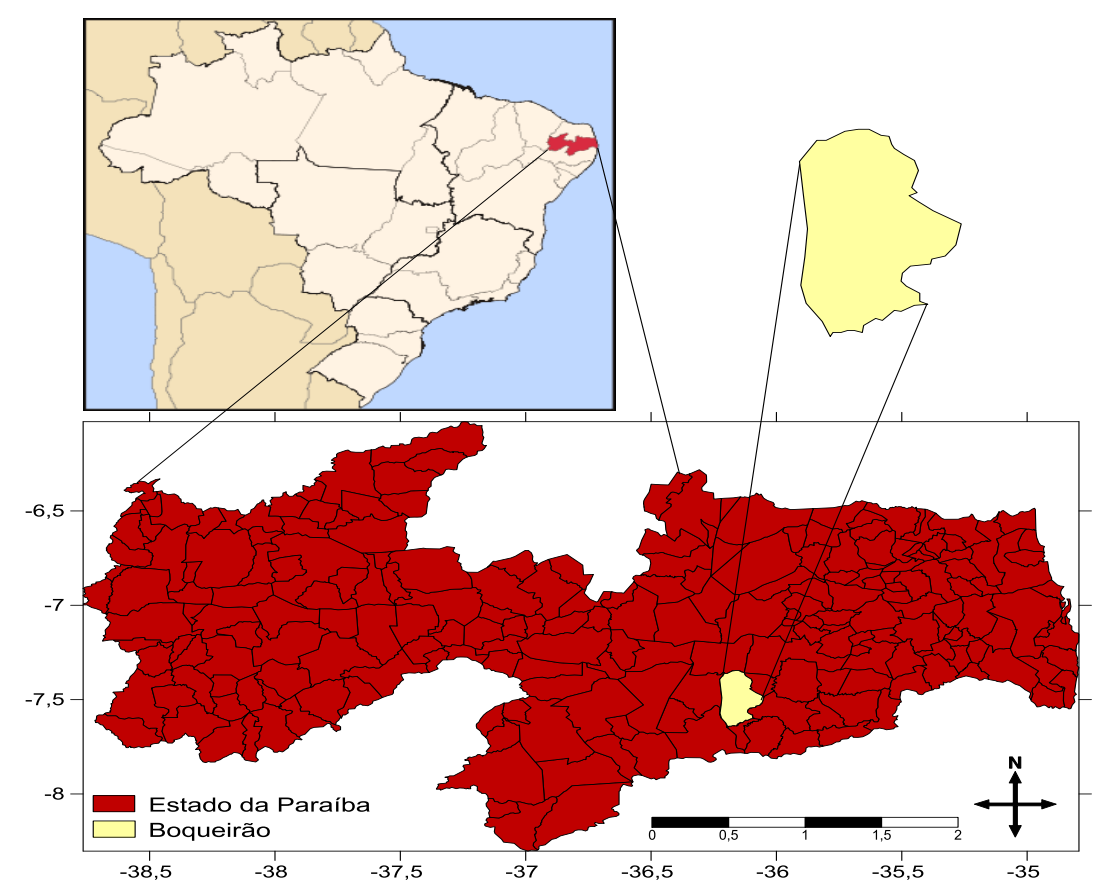

Figura 1. Localização geográfica do município de Boqueirão-PB.

Os dados pluviométricos foram obtidos na Agência Executiva de Gestão das Águas do Estado da Paraíba (AESA). Foram utilizados dados de totais mensais precipitados para o período de 1963-2014.

A caracterização do regime de precipitação pluvial foi realizada por meio do ajuste das séries empíricas à distribuição gama. A função distribuição de probabilidade gama é visualizada na Equação 1.

$$
g(x)=\frac{1}{\beta^{\alpha} \Gamma(\alpha)} x^{\alpha-1} e^{-x / \beta}
$$

em que:

$\alpha>0=$ parâmetro de forma da distribuição gama;

$\beta>0=$ parâmetro de escala da distribuição gama; 


$$
\begin{aligned}
& \chi>0=\text { total de precipitação; e } \\
& \Gamma(\alpha)=\text { função gama. }
\end{aligned}
$$

Para estimativa dos parâmetros $\alpha$ e $\beta$, utilizaram-se as soluções de máxima verossimilhança (Equações 2, 3 e 4).

$$
\begin{aligned}
& \alpha=\frac{1}{4 A}\left(1+\sqrt{\left.1+\frac{4 A}{3}\right)}\right. \\
& A=\ln (\bar{x})-\frac{\sum_{i}^{n} x_{i}}{n} \\
& \beta=\frac{\bar{x}}{\alpha}
\end{aligned}
$$

em que:

$$
\begin{aligned}
& \bar{x}=\text { média aritmética da precipitação pluvial }(\mathrm{mm}) \\
& \ln =\text { logaritmo neperiano; e } \\
& \mathrm{n}=\text { número de observações da amostra. }
\end{aligned}
$$

Após a estimativa de $\mathrm{g}(\mathrm{x})$ para as séries de precipitações investigadas, o IPP pôde ser calculado através da Equação 5.

$$
H(x)=q+(1-q) G(x)
$$

em que:

$H(x)=$ distribuição de probabilidade cumulativa;

$\mathrm{q}=$ probabilidade de ocorrência de valores nulos (zeros); e

$\mathrm{G}(\mathrm{x})=$ distribuição cumulativa teórica.

Se $\mathrm{m}$ for o número de zeros numa série de precipitação, então $q=\mathrm{m} / \mathrm{n}$. em que:

m - número de observações com chuva igual a zero; e

$\mathrm{n}$ - número de observações com chuva maior do que zero.

$\mathrm{H}(\mathrm{x})$ foi então transformada em uma variável normal (valor final do IPP) por meio das equações desenvolvidas por Abramowitz e Stegun (1965). A relação entre as distribuições de probabilidade gama e normal é apresentada nas Equações 6, 7, 8 e 9.

$$
\begin{aligned}
& Z=S P I=-\left(t-\frac{c_{0}+c_{1} t+c_{2} t^{2}}{1+d_{1} t+d_{2} t^{2}+d_{3} t^{3}}\right) \text { Para } 0<H(x) \leq 0,5 \\
& Z=S P I=+\left(t-\frac{c_{0}+c_{1} t+c_{2} t^{2}}{1+d_{1} t+d_{2} t^{2}+d_{3} t^{3}}\right) \text { Para } 0,5<H(x) \leq 1,0
\end{aligned}
$$

em que:

$$
\mathrm{c} 0=2,515 ; \mathrm{c} 1=0,803 ; \mathrm{c} 2=0,010 ; \mathrm{d} 1=1,433 ; \mathrm{d} 2=0,189 ; \mathrm{e} \mathrm{d} 3=0,001
$$

\section{IPABH}




$$
\begin{gathered}
t=\sqrt{\ln \left(\frac{1}{(H(x))^{2}}\right)} \text { Para } 0<H(x) \leq 0,5 \\
\mathrm{e}_{t}=\sqrt{\ln \left(\frac{1}{(1-H(x))^{2}}\right)} \text { Para } 0,5<H(x) \leq 1,0
\end{gathered}
$$

Os valores do IPP com suas categorias estão disponíveis na Tabela 1. Em função de serem mais graves, analisou-se apenas as secas severas e extremas, pois são estas que causam os impactos sociais, econômicos e ambientais mais intensos.

Tabela 1. Valores do IPP e categorias de chuvas e de secas.

\begin{tabular}{cl}
\hline Valores do IPP & \multicolumn{1}{c}{ Categoria } \\
\hline$\geq 2,00$ & Chuva extrema \\
1,50 a 1,99 & Chuva severa \\
1,00 a 1,49 & Chuva moderada \\
0 a 0,99 & Chuva fraca \\
0 a $-0,99$ & Seca fraca \\
$-1,00$ a $-1,49$ & Seca moderada \\
$-1,50$ a $-1,99$ & Seca severa \\
$\leq-2,00$ & Seca extrema \\
\hline
\end{tabular}

Fonte: Mckee et al. (1993).

Para análise dos impactos foram utilizados dados obtidos no Instituto Brasileiro de Geografia e Estatística (IBGE, 2015). Na agricultura, foram analisados: Área plantada (ha), área colhida (ha), quantidade produzida (ton), rendimento médio ( $\mathrm{kg} / \mathrm{ha}$ ) e valor da produção (R\$) da banana, caju, coco, goiaba, laranja, limão, mamão, maracujá, manga, algodão herbáceo, batata-doce, cebola, fava, feijão, milho e tomate dos anos de 2011, 2012 e 2013 . O ano de 2014 não foi incluído porque os dados ainda não estavam disponíveis.

Na pecuária foi analisado o efetivo dos rebanhos de asininos, bovinos, caprinos, equinos, galos e galinhas; muares, ovinos e suínos dos anos de 2011, 2012, 2013 e 2014.

Com relação aos produtos agropecuários, foram utilizadas as variáveis: leite de vaca (quantidade), leite de vaca (valor da produção R\$), ovos de galinha (quantidade) e ovos de galinha (valor da produção R\$) dos anos de 2011, 2012, 2013 e 2014.

A opção de analisar apenas os impactos da última seca foi devido à ausência de dados de secas anteriores. O ano de 2011 foi incluído porque foi chuvoso e pelo interesse em fazer um comparativo entre 2011 (ano chuvoso) e 2012-2014 (anos secos).

\section{RESULTADOS E DISCUSSÃO}

O IPP na escala de 3 meses identificou cinco secas, quatro severas e uma extrema. As durações das severas variaram de 1 a 8 meses, merecendo destaque a mais longa ( 8 meses) por ter acontecido recentemente (setembro de 2012 até abril de 2013). Esta reduziu drasticamente a produção das culturas. Durante esse período as famílias ficaram com a alimentação limitada em quantidade e qualidade, e a dieta das famílias mais vulneráveis ficou reduzida 
principalmente ao milho e feijão. A seca extrema ocorreu entre abril e setembro de 1998, durou 6 meses e atingiu o IPP médio de - 2,19, sendo outra seca que causou muitos impactos negativos na zona urbana e rural do município (Tabela 2A).

Com relação ao IPP na escala de 6 meses, mais uma vez a seca de 1998 se destaca, tendo início em abril de 1998 e término em dezembro do mesmo ano, com duração de 9 meses, IPP médio de - 2,11 e classificação extrema, a única nesta categoria. Outra seca que se destacou foi a de 2012-2013, pois apresentou a maior duração (12 meses). É importante observar as secas que foram identificadas pelas escalas anteriores e persistem nas demais, isso indica que elas merecem destaque maior, pois permaneceram atuando por mais tempo. As demais secas severas ocorreram entre fevereiro e março de 1970, fevereiro e maio de 1971, agosto de 1980 e janeiro de 1981 e outubro de 1981 e janeiro de 1982. Os IPPs médios destas secas variaram de - 1,53 até - 1,90 (Tabela 2B).

A quantidade de secas identificadas foi reduzida na escala de 9 meses, porém houve aumento na duração. Nessa escala foram identificadas cinco secas, três severas e duas extremas. As severas aconteceram entre 1980-1981, 1983-1984 e 2012-2013, todas estas atingiram o semiárido nordestino, em alguns lugares duraram mais tempo e causaram mais impactos negativos, em outros foram de menor intensidade. Já as secas extremas atuaram de janeiro a fevereiro de 1982, com duração de 2 meses e IPP médio de - 2,28. Com relação à outra seca extrema, novamente ela se repetiu, iniciando em março de 1998 e terminando em março de 1999 (Tabela 2C). É possível observar um aumento da duração desta seca se comparada com as das escalas anteriores, a categoria de seca extrema se manteve para os IPPs de 3, 6 e 9 meses.

Com o IPP na escala de 12 meses foram identificadas duas secas, ambas severas, uma entre 1998-2000 e a outra durante 2012-2013. A primeira teve duração de 21 meses e atingiu o IPP médio de - 1,81; a segunda durou 10 meses, com IPP médio de - 1,55. Resultados próximos a estes foram obtidos para a escala de 24 meses, duas secas identificadas, uma severa (maio de 1980 a fevereiro de 1981) e a outra extrema (fevereiro de 1999 a junho de 2000) e tiveram durações de 10 e 17 meses, respectivamente (Tabela 2D e E). Quando se aumentou a escala, muitas secas identificadas pelos IPPs anteriores foram agrupadas em escalas maiores, por exemplo, secas dos IPPs de 3, 6 e 9 meses se agruparam e formaram secas nos IPPs de 12 e 24 meses, quando isso acontece, deve-se dar maior importância as secas que permanecem nas escalas maiores.

As secas mais graves no município de Boqueirão ocorreram nos anos de 1980-1982, 19982000 e 2012-2013. Destas, a mais impactante foi a de 1998, isso ocorreu devido a um forte El Niño, que é considerado um dos mais intensos dos últimos 100 anos. Em anos de El Niño, quando as águas superficiais da bacia do Pacífico estão mais aquecidas que o normal, pode haver inibição da formação de nuvens e, consequentemente, diminuição das chuvas, principalmente na Região Norte do Nordeste. Além dos outros impactos, essa seca reduziu drasticamente a produção agropecuária e o abastecimento de água da região.

Com a escassez de chuvas nos municípios a montante do açude Epitácio Pessoa durante 1998-2000, o manancial não foi reabastecido. Isso fez com que fosse adotado o racionamento de água nos anos de 1998, 1999 e 2001, começando quando o açude tinha um volume próximo de 100.000.000 de $\mathrm{m}^{3}$ de água. Este foi um período crítico, haja vista que suas águas abastecem diversos municípios, entre eles, Campina Grande. A situação foi ainda mais agravada devido à irrigação no entorno do açude, pois esta é a atividade que consome mais água. Mesmo com a proibição pelo poder público, ela continuou sendo realizada de forma clandestina. 
Tabela 2. Quantidade, período, duração, IPP médio e categoria de secas obtidas pelos IPPs de $3,6,9,12$ e 24 meses para o município de Boqueirão-PB.

\begin{tabular}{cllccl}
\hline \multicolumn{5}{c}{ Escala de 3 meses (A) } \\
\hline Quantidade & \multicolumn{1}{c}{ Início } & \multicolumn{1}{c}{ Fim } & $\begin{array}{c}\text { Duração } \\
\text { (meses) }\end{array}$ & $\begin{array}{c}\text { IPP } \\
\text { médio }\end{array}$ & Categoria \\
\hline 1 & dezembro 1970 & março 1971 & 4 & $-1,55$ & Seca severa \\
2 & abril 1998 & setembro 1998 & 6 & $-2,19$ & Seca extrema \\
3 & janeiro 2005 & janeiro 2005 & 1 & $-1,67$ & Seca severa \\
4 & maio 2012 & julho 2012 & 3 & $-1,69$ & Seca severa \\
5 & setembro 2012 & abril 2013 & 8 & $-1,60$ & Seca severa \\
\hline
\end{tabular}

Escala de 6 meses $(\mathbf{B})$

\begin{tabular}{cllccl}
\hline Quantidade & \multicolumn{1}{c}{ Início } & \multicolumn{1}{c}{ Fim } & $\begin{array}{c}\text { Duração } \\
\text { (meses) }\end{array}$ & $\begin{array}{c}\text { IPP } \\
\text { médio }\end{array}$ & Categoria \\
\hline 1 & fevereiro 1970 & março 1970 & 2 & $-1,59$ & Seca severa \\
2 & fevereiro 1971 & maio 1971 & 4 & $-1,53$ & Seca severa \\
3 & agosto 1980 & janeiro 1981 & 6 & $-1,62$ & Seca severa \\
4 & outubro 1981 & janeiro 1982 & 4 & $-1,90$ & Seca severa \\
5 & abril 1998 & dezembro 1998 & 9 & $-2,11$ & Seca extrema \\
6 & agosto 2012 & julho 2013 & 12 & $-1,55$ & Seca severa \\
\hline
\end{tabular}

Escala de 9 meses $(\mathbf{C})$

\begin{tabular}{cllccl}
\hline Quantidade & \multicolumn{1}{c}{ Início } & \multicolumn{1}{c}{ Fim } & $\begin{array}{c}\text { Duração } \\
\text { (meses) }\end{array}$ & $\begin{array}{c}\text { IPP } \\
\text { médio }\end{array}$ & Categoria \\
\hline 1 & agosto 1980 & janeiro 1981 & 6 & $-1,60$ & Seca severa \\
2 & janeiro 1982 & fevereiro 1982 & 2 & $-2,28$ & Seca extrema \\
3 & dezembro 1983 & fevereiro 1984 & 3 & $-1,56$ & Seca severa \\
4 & março 1998 & março 1999 & 13 & $-2,00$ & Seca extrema \\
5 & novembro 2012 & junho 2013 & 8 & $-1,68$ & Seca severa \\
\hline
\end{tabular}

Escala de 12 meses (D)

\begin{tabular}{cccccc}
\hline Quantidade & Início & Fim & $\begin{array}{c}\text { Duração } \\
\text { (meses) }\end{array}$ & $\begin{array}{c}\text { IPP } \\
\text { médio }\end{array}$ & Categoria \\
\hline 1 & maio 1998 & janeiro 2000 & 21 & $-1,81$ & Seca severa \\
2 & dezembro 2012 & setembro 2013 & 10 & $-1,55$ & Seca severa \\
\hline Quantidade & Início & Escala de 24 meses (E) & & \\
\hline 1 & maio 1980 & Fim & $\begin{array}{c}\text { Duração } \\
\text { (meses) }\end{array}$ & $\begin{array}{c}\text { IPP } \\
\text { médio }\end{array}$ & Categoria \\
\hline 2 & fevereiro 1999 & junho 2000 & 10 & $-1,80$ & Seca severa \\
& & & 17 & $-2,25$ & Seca extrema \\
\hline
\end{tabular}

A seca de 2012-2016 tem causado muitos impactos à população que depende das águas do Epitácio Pessoa. Com precipitações abaixo da média nas bacias hidrográficas do Alto Paraíba e Taperoá durante esses anos, o volume vem sendo reduzido dia após dia. Atualmente (dezembro de 2016) o açude está com 5\% de sua capacidade. E mais uma vez o racionamento 
se fez presente, tendo início em dezembro de 2014, passando por 2015 e 2016. Inclusive, se não houver recarga brevemente, a tendência é de que o sistema seja desligado nos próximos 2, 3 meses, deixando diversas cidades sem água.

Pode ser observado na Figura 2 A, B, C, D e E que a correlação entre precipitação (mm) e área plantada (ha), área colhida (ha), quantidade produzida (ton), rendimento médio ( $\mathrm{kg} / \mathrm{ha}$ ) e valor da produção $(\mathrm{R} \$)$ da banana, caju, coco, goiaba, laranja, limão, mamão, maracujá e manga, que são culturas permanentes, nem sempre ocorre. Isso é explicado porque grande parte dessas culturas são irrigadas com a água do açude Epitácio Pessoa. Ou seja, quando aconteceu a seca, o manancial tinha uma satisfatória quantidade de água, o que fez com que os impactos nas culturas agrícolas permanentes não fossem tão intensos durante 2012-2013.

As maiores áreas plantadas e colhidas (ha) foram com banana, mamão e maracujá. Em 2011, 2012 e 2013 foram utilizados na plantação: 155, 155 e 200 ha; 25, 25 e 8 ha; e 8, 20 e 30 ha com banana, mamão e maracujá, respectivamente. Com relação à área colhida, foram obtidos os seguintes valores: banana, 155, 155 e 155 ha; mamão, 25, 25 e 8 ha; e maracujá, 8, 20 e 30 ha. É possível observar que mesmo com precipitação abaixo da média em 2012 e 2013, as culturas do mamão e maracujá tiveram toda a área que foi plantada, colhida (Figura 2 A e B). Isso foi possível por causa da irrigação.

Houve redução da quantidade produzida (ton) de banana, caju, coco, goiaba e manga durante 2012-2013. O contrário ocorreu com as demais culturas. Isso é explicado por que a bananeira, cajueiro, coqueiro, goiabeira e mangueira são mais tolerantes ao déficit hídrico, com isso, menos água foi ofertada a estas culturas e mais a laranjeira, limoeiro, mamoeiro e maracujazeiro (Figura $2 \mathrm{C}$ ).

Também houve redução do rendimento médio por hectare das culturas em função da seca. Reduções lineares durante os anos ocorreram para banana, caju, coco e goiaba. O rendimento médio em 2011, 2012 e 2013 foi, respectivamente, banana, $16.000,16.000$ e $11.613 \mathrm{~kg} / \mathrm{ha}$; caju, 400, 200 e $200 \mathrm{~kg} / \mathrm{ha}$; coco, 20.000, 8.000 e $5.100 \mathrm{~kg} / \mathrm{ha}$; e goiaba, $8.000,6.000$ e $5.200 \mathrm{~kg} / \mathrm{ha}$ (Figura 2 D). As outras culturas tiveram menores reduções em função do que já foi discutido nos parágrafos anteriores. Todas as culturas tiveram redução do valor da produção, exceto laranjeira e maracujazeiro. Os valores para estas culturas durante 2011, 2012 e 2013 foram: $18.000,30.000$ e $\mathrm{R} \$ 62.000 ; 56.000,180.000$ e $\mathrm{R} \$ 270.000$, respectivamente, (Figura $2 \mathrm{E}$ ). Isso ocorreu porque na falta de alguns produtos, houve aumento dos preços, o que se conhece por lei da oferta e da procura. Quando a oferta de produtos é maior que a procura, a tendência é de que ocorra a redução de preços. Já nos períodos em que a demanda passa a superar a oferta, a tendência é o aumento dos preços.

A seca em 2012 e 2013 impactou severamente as culturas agrícolas temporárias no município de Boqueirão. Pode ser observado na Figura 3 a correlação entre precipitação $(\mathrm{mm})$ e área plantada (ha), área colhida (ha), quantidade produzida (ton), rendimento médio ( $\mathrm{kg} / \mathrm{ha}$ ) e valor da produção $(\mathrm{R} \$)$.

Com exceção da batata-doce, todas as outras culturas tiveram redução de área plantada (ha) em 2012 e 2013. A maior ocorreu com as culturas de feijão e milho, isso causou impactos gravíssimos, pois são alimentos que garantem a subsistência da população. Já a batata-doce não teve redução de área plantada porque a cultura era cultivada nas vazantes dos açudes, principalmente do Epitácio Pessoa, (Figura 3 A).

A área colhida está diretamente relacionada com a plantada. A redução da área plantada em 2012 e 2013 resultou em menor área colhida. Batata-doce, cebola e tomate tiveram toda a área que foi plantada, colhida. O oposto aconteceu com algodão herbáceo, fava, feijão e milho. Para estas culturas as áreas colhidas em 2011, 2012 e 2013 foram: algodão herbáceo, 20, 0 e 0 ha; fava, 100, 0 e 10 ha; feijão, 700, 0 e 40 ha; e milho, 1.000, 100 e 20 ha, respectivamente, (Figura 3 B). 


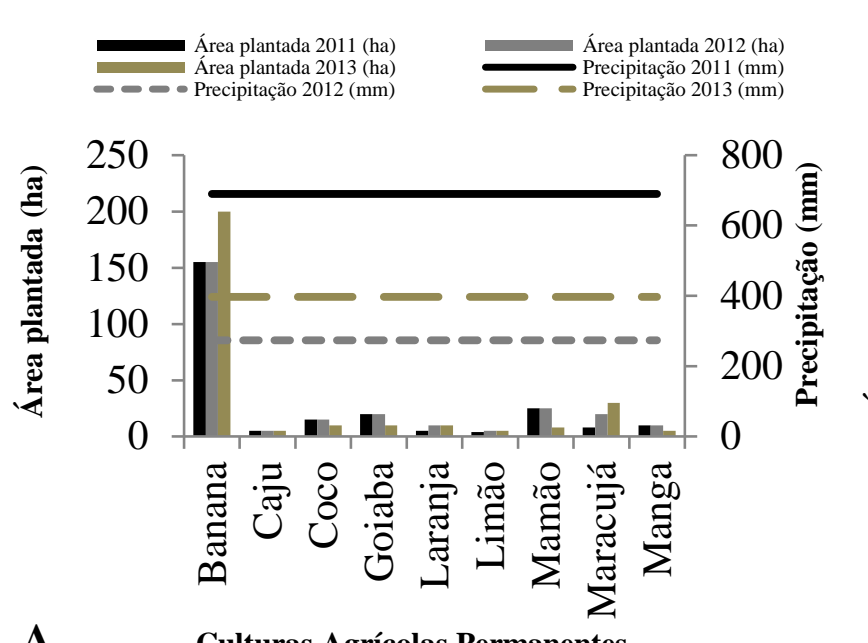

A Culturas Agrícolas Permanentes

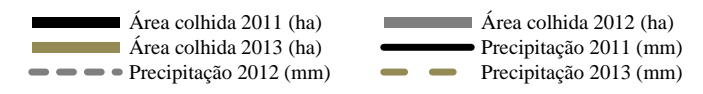

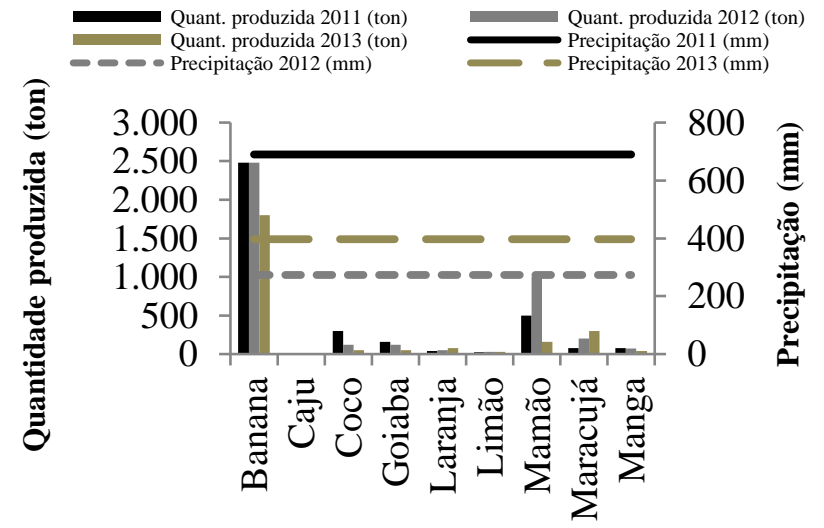

Culturas Agrícolas Permanentes

C

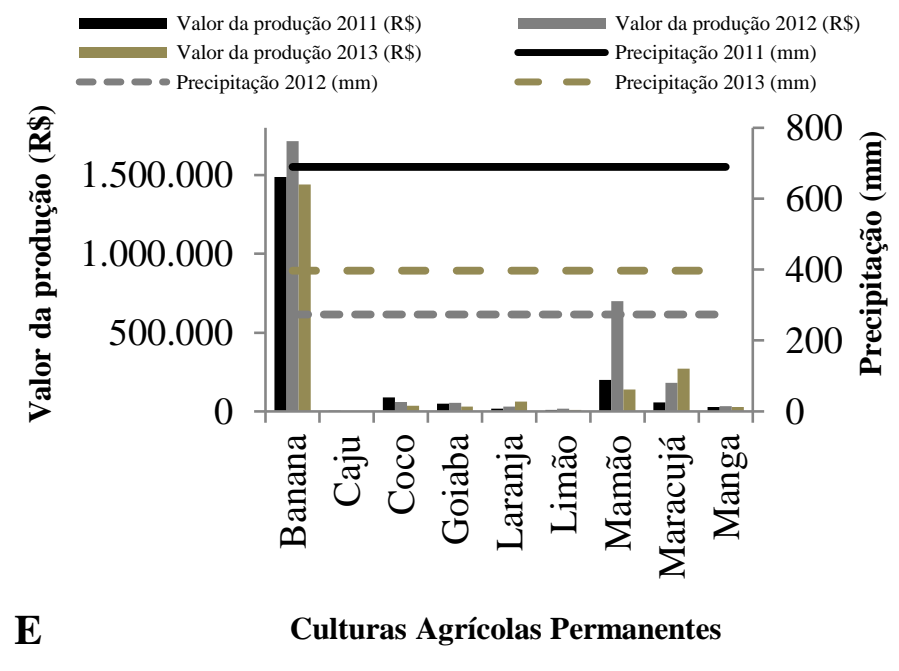

Figura 2. Impactos da seca de 2012-2013 na área plantada (ha) (A), área colhida (ha) (B), quantidade produzida (ton) $(\mathrm{C})$, rendimento médio $(\mathrm{kg} / \mathrm{ha})(\mathrm{D})$ e valor da produção $(\mathrm{R} \$)(\mathrm{E})$ das culturas agrícolas permanentes no município de Boqueirão-PB.

A quantidade produzida (ton) teve grande redução. Os valores foram os seguintes em 2011, 2012 e 2013: algodão herbáceo, 16, 0 e 0 ton; cebola, 2.000, 4.800 e 1.000 ton; fava, 30, 0 e

Rev. Ambient. Água vol. 12 n. 2 Taubaté - Mar. / Apr. 2017 
1 ton; feijão, 280, 0 e 6 ton; milho, 600, 12 e 5 ton; e tomate, 3.200, 5.250 e 2.800 ton, respectivamente, (Figura 3 C). A redução para valores próximos de 0 ton em 2012 e 2013 nas culturas de algodão, fava, feijão e milho é devido as mesmas serem totalmente dependentes das chuvas que caem durante janeiro-maio de cada ano.
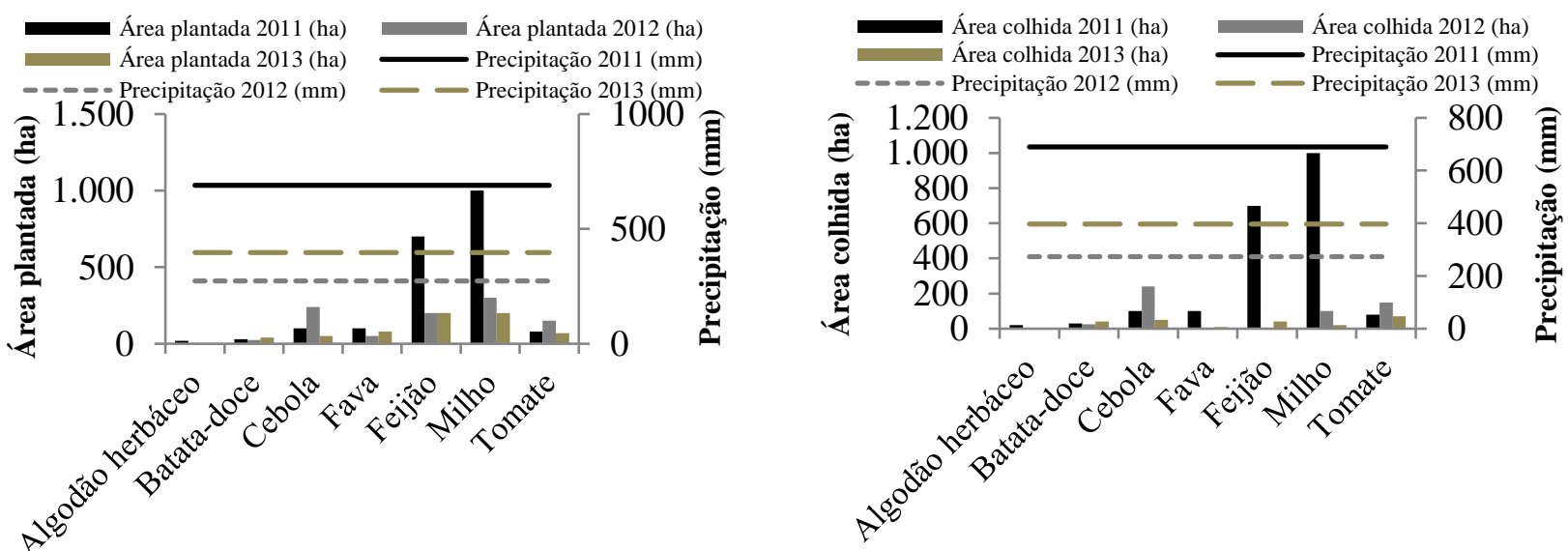

Culturas Agrícolas Temporárias

Culturas Agrícolas Temporárias

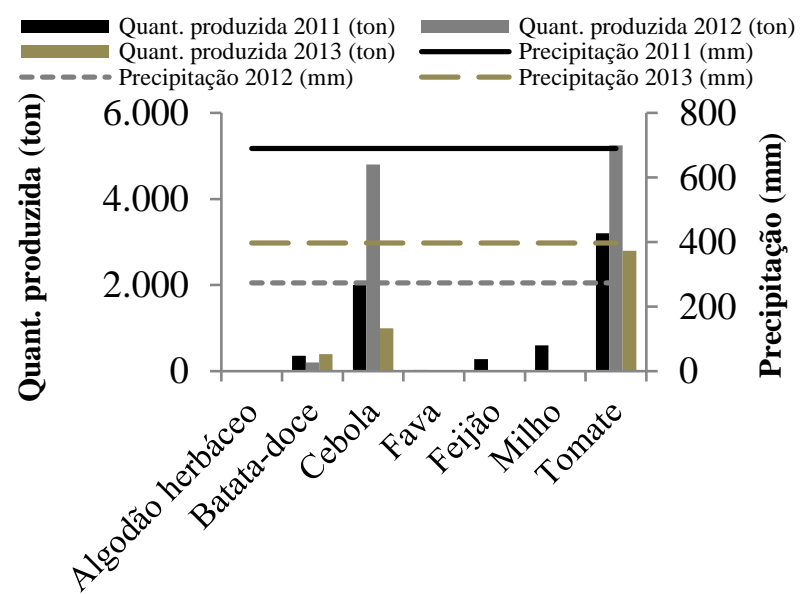

Culturas Agrícolas Temporárias

C

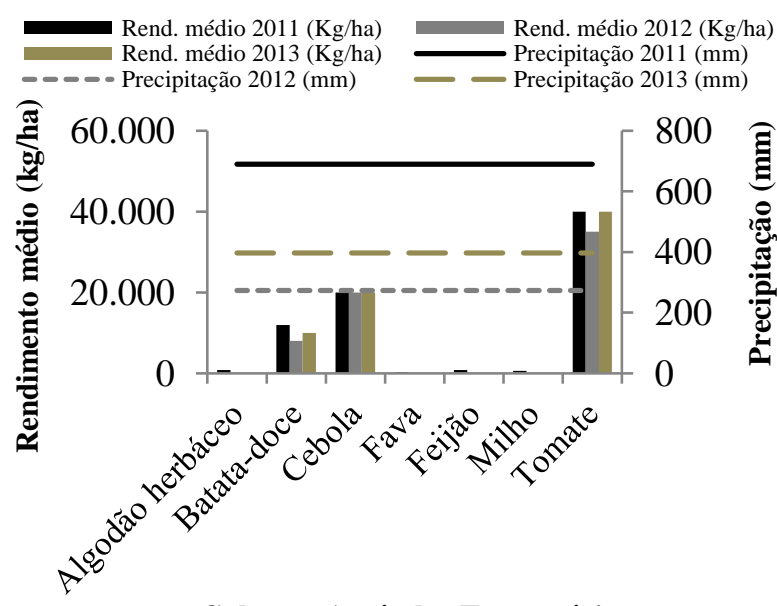

Culturas Agrícolas Temporárias

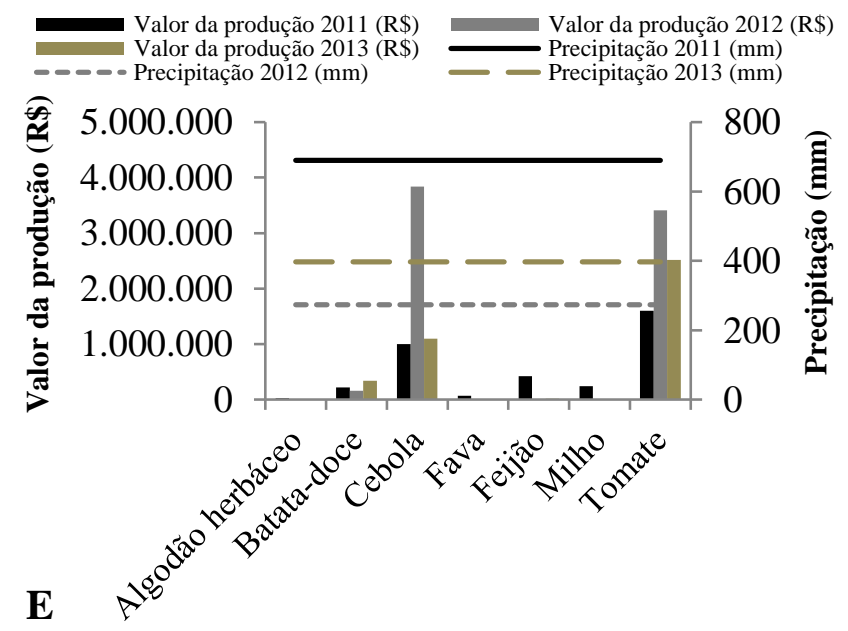

Culturas Agrícolas Temporárias

Figura 3. Impactos da seca de 2012-2013 na área plantada (ha) (A), área colhida (ha) (B), quantidade produzida (ton) $(\mathrm{C})$, rendimento médio $(\mathrm{kg} / \mathrm{ha})(\mathrm{D})$ e valor da produção $(\mathrm{R} \$)(\mathrm{E})$ das culturas agrícolas temporárias no município de Boqueirão-PB. 
Houve redução do rendimento médio por hectare das culturas em função da seca. $\mathrm{O}$ algodão herbáceo e a fava não produziram nenhum $\mathrm{kg} / \mathrm{ha}$ durante $2012 \mathrm{e} 2013$. A maior redução ocorreu com algodão herbáceo, batata-doce, fava, feijão e milho (Figura 3 D). A Figura 3 E evidencia a redução do valor da produção entre 2011, 2012 e 2013. Os valores foram os seguintes: algodão herbáceo, 24.000, 0 e R\$ 0; batata-doce, 216.000, 160.000 e R \$340.000; cebola, 1.000.000, 3.840.000 e R\$ 1.100.000; fava, 66.000, 0 e R\$ 5.000; feijão, 424.000, 0 e $\mathrm{R} \$$ 13.000; milho, 240.000, 8.000 e R \$ 4.000; e tomate, 1.600.000, 3.413.000 e R\$ 2.520.000. Esse é o tamanho do impacto econômico e social que as famílias tiveram.

Ocorreu redução do efetivo de animais durante a seca, principalmente em 2012 e 2013. Com a precipitação próxima da média em 2014, a quantidade de animais voltou a ter valores próximos ou superiores aos de 2011.

A menor redução ocorreu com os asininos, equinos e muares. Isso porque a quantidade desses animais era pequena e a maioria dos criadores utilizavam para realizar atividades no meio rural, assim não podiam se desfazer dos mesmos.

A quantidade de aves domésticas (galos e galinhas) e suínos foi reduzida entre os dois primeiros anos, havendo aumento no terceiro e quarto. Os números em 2011, 2012, 2013 e 2014 foram os seguintes: galos e galinhas, 13.900, 13.400, 18.050 e 19.000 animais; e suínos, 750, 725, 987 e 1.400 animais, respectivamente, (Figura 4). O aumento da quantidade de galos, galinhas e suínos mesmo durante os anos secos de 2013 e 2014 é muito importante, pois esses animais contribuem bastante para a alimentação e economia das famílias.

Os bovinos apresentaram o segundo maior rebanho, seguido por caprinos e ovinos. A quantidade de bovinos, caprinos e ovinos criados em 2011, 2012, 2013 e 2014 foi de 10.200, 8.800, 8.894 e 10.000 animais; 9.088, 6.500, 8.040 e 9.000 animais; e 3.660, 3.000, 4.831 e 7.000 animais, respectivamente, (Figura 4). Com a redução de água e alimento durante a seca, alguns animais foram vendidos por preços abaixo do valor de mercado, outros morreram de fome e sede. A maior quantidade de bovinos em relação aos caprinos e ovinos é devido o município ser uma bacia leiteira de grande importância, com várias associações e cooperativas de beneficiamento do leite de vaca. Também se destaca na pecuária de corte. Já o grande número de caprinos e ovinos é por causa de sua adaptabilidade às condições adversas do semiárido e facilidade na comercialização.

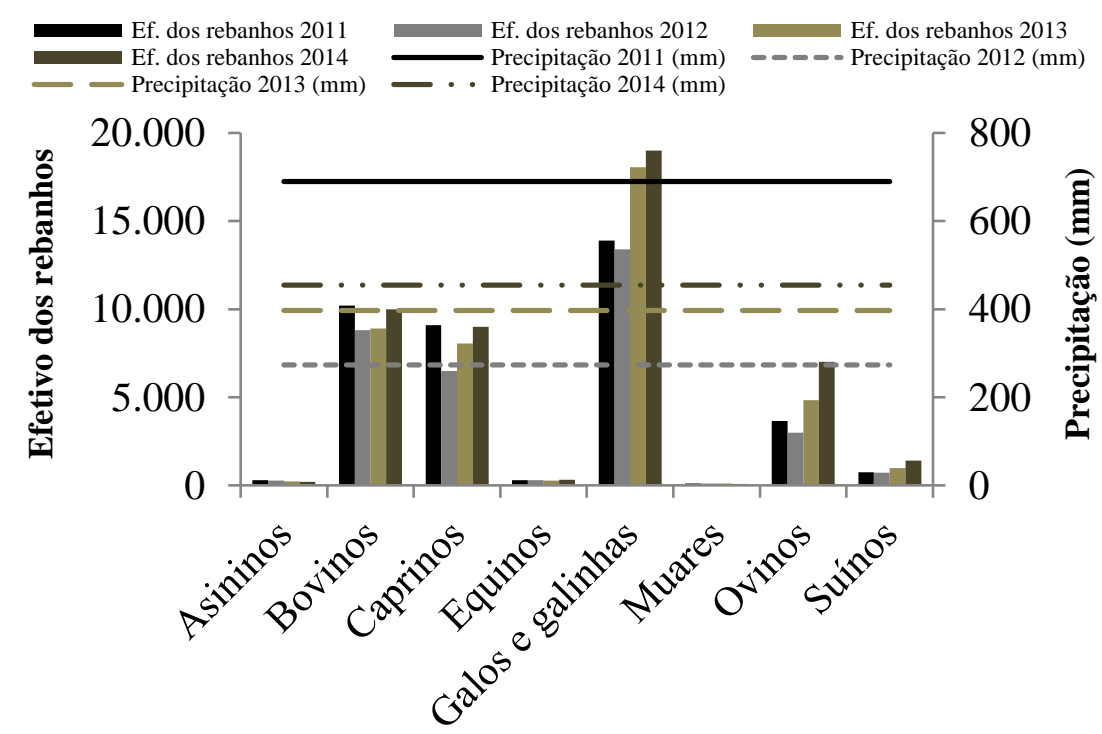

\section{Rebanhos}

Figura 4. Impactos da seca de 2012-2014 no efetivo dos rebanhos no município de Boqueirão-PB. 
A seca em 2012 dificultou o desenvolvimento das pastagens, influenciou fortemente o efetivo do rebanho bovino, causando redução na produção do leite. Com a diminuição de bovinos em 2012, a quantidade de leite produzido reduziu, passando de 2.808 .000 litros em 2011 para 2.600.000 em 2012. Mesmo com a seca em 2013 e 2014, houve aumento da quantidade de leite produzido em relação ao ano de 2011, isso ocorreu em função da compra de farelo de trigo, milho e algodão para alimentar os animais (Figura $5 \mathrm{~A}$ ).

A seca reduziu a quantidade de ovos produzidos. A produção de ovos em 2011 foi de 24.000 dúzias, 22.000 em 2012, 23.000 em 2013 e 23.000 em 2014 (Figura 5 B). Além da redução das espécies vegetais, a pequena produção de milho nas propriedades em função da falta de chuva reduziu a produção das galinhas. A redução da quantidade de ovos ocorreu mesmo com o programa de subsidio de milho da CONAB, onde os criadores podiam comprar a saca de 60 quilos pelo preço de $\mathrm{R} \$ 18,10$ em 2012. Só que houve aumento nos valores do milho comercializado em 2013 e 2014, inviabilizando a compra.

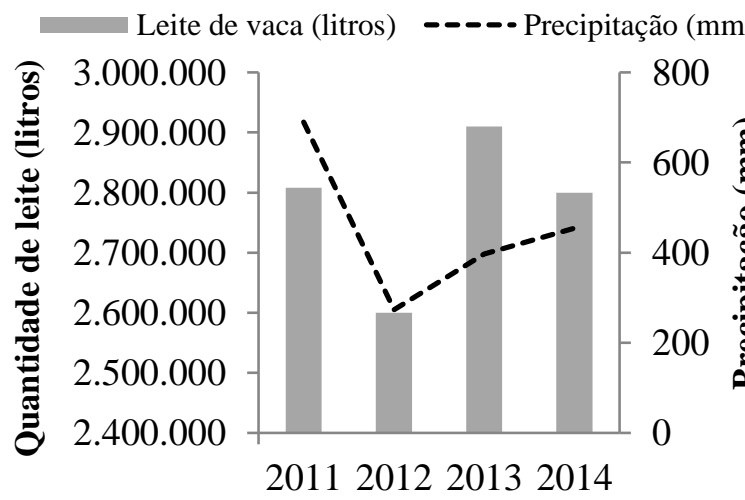

A

Anos

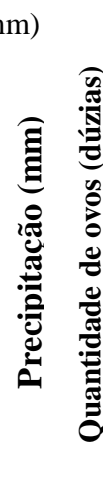

B

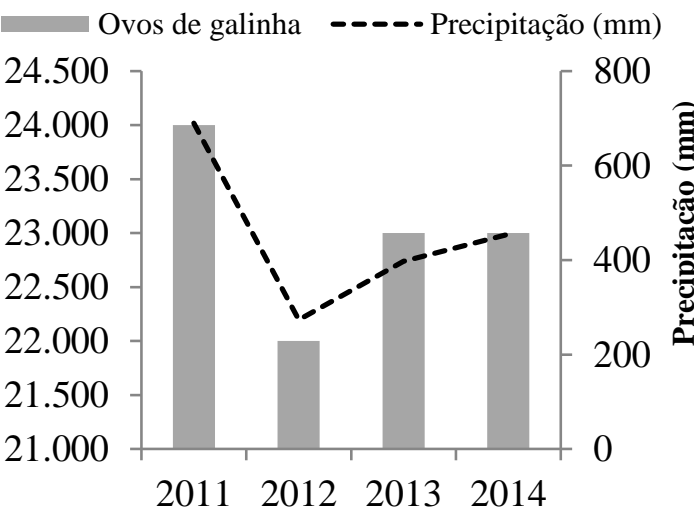

Anos

Figura 5. Impactos da seca de 2012-2014 na quantidade de leite de vaca (A) e de ovos de galinha (B) no município de Boqueirão-PB.

A diminuição das chuvas e da quantidade de leite e ovos produzidos em 2012, 2013 e 2014 provocou aumento do valor obtido com a venda de leite e ovos, e isso está diretamente relacionado com a lei da oferta e da procura, (Figura 6 A e B).

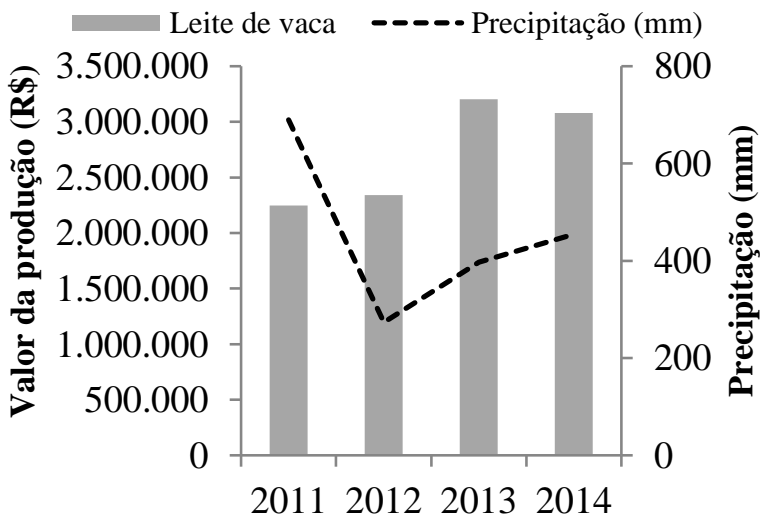

A

Anos

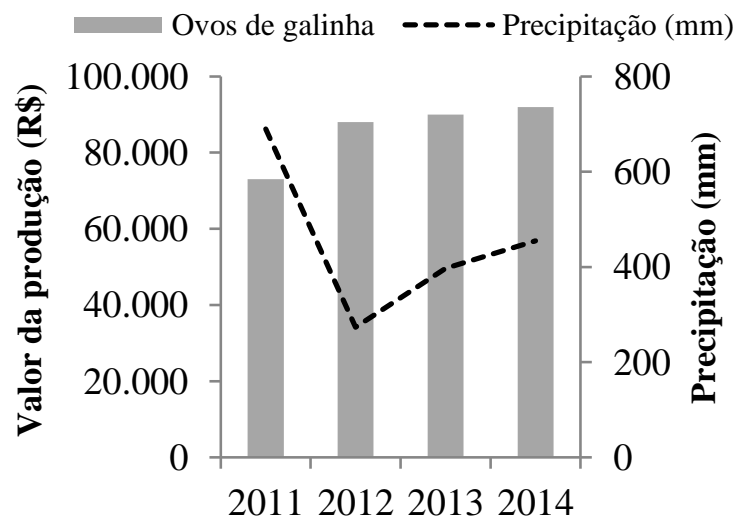

B

Anos

Figura 6. Impactos da seca de 2012-2014 no valor da produção do leite de vaca (A) e de ovos de galinha (B) no município de Boqueirão-PB. 


\section{CONCLUSÕES}

As secas mais graves no município de Boqueirão ocorreram nos anos de 1980-1982, 1998-2000 e 2012-2013. Destas, a mais impactante foi a de 1998-2000. Além de muitos outros impactos, essa seca reduziu drasticamente a produção agropecuária e o abastecimento de água da região.

Os impactos mais intensos durante a seca 2012-2014 ocorreram em 2012 e 2013. As culturas agrícolas temporárias foram mais impactadas do que as permanentes. Isso ocorreu porque a maioria das permanentes eram irrigadas, diferente das temporárias. A maior redução de animais também ocorreu durante 2012 e 2013, com uma relação direta entre precipitação e efetivo dos rebanhos. Não houve redução do valor da produção em função das secas.

Para redução dos impactos que ocorreram, é necessário aumentar o número de cisternas, principalmente a calçadão. É necessário também perfurar poços e construir açudes, além de fazer manutenção nos que estejam com capacidade reduzida ou desativados, construir barragens subterrâneas e tanques naturais, terminar e colocar em funcionamento a transposição do Rio São Francisco, incentivar a gestão dos recursos hídricos, criar programas que visem o fortalecimento da agricultura familiar, incentivando a fenação, silagem na criação animal e fomentar o plantio de culturas adaptadas à região.

\section{REFERÊNCIAS}

ABRAMOWITZ, M.; STEGUN, I. A. (Eds.). Handbook of Mathematical Functions with formulas, graphs, and mathematical tables. New York: Dover Publications, 1965. $1046 \mathrm{p}$.

BORDI, I.; FRAEDRICH, F.; SUTERA, A. Observed drought and wetness trends in Europe: an update. Hydrology and Earth System Sciences, v. 13, p. 1519-1530, 2009. http://dx.doi.org/10.5194/hess-13-1519-2009.

BRASIL. As secas do Nordeste: uma abordagem histórica de causas e efeitos. Recife: SUDENE, 1981.

FARIAS, A. A.; SOUZA, J. T. A.; SOUSA, F. A. S. Identificação e análise de secas severas e extremas no município de Taperoá-PB. Revista Brasileira de Geografia Física, v. 07, n. 05, p. 818-826, 2014.

FISCHER, T.; GEMMER, M.; SU, B.; SCHOLTEN, T. Hydrological long-term dry and wet periods in the Xijiang River basin, South China. Hydrology and Earth System Sciences, v. 17, p. 135-148, 2013. http://dx.doi.org/10.5194/hess-17-135-2013

GUTTMAN, N. Accepting the standardised precipitation index: a calculation algorithm. Journal of the American Water Resources Association, v. 35, p. 311-322, 1999. http://dx.doi.org/10.1111/j.1752-1688.1999.tb03592.x

HAYES, M. J.; SVOBODA, M. D.; MURO, N. A.; WIDHALM, M. The Lincoln declaration on drought indices: universal meteorological drought index recommended. Bulletin of the American Meteorological Society, v. 92, p. 485-488, 2011. http://dx.doi.org/10.1175/2010BAMS3103.1.

INSTITUTO BRASILEIRO DE GEOGRAFIA E ESTATÍSTICA - (IBGE). Produção agrícola e pecuária municipal. 2015. Disponível em: https://goo.gl/jRKqfy. Acesso em: 13 out. 2015. 
KEYANTASH, J.; DRACUP, J. A. The quantification of drought: an evaluation of drought indices. Bulletin of the American Meteorological Society, v. 83, p. 1167-1180, 2002. http://dx.doi.org/10.1175/1520-0477(2002)083\%3C1191:TQODAE\%3E2.3.CO;2

LI, B.; RODELL, M. Evaluation of a model-based groundwater drought indicator in the conterminous U. S. Journal of Hydrology, v. 526, p. 78-88, 2015. http://dx.doi.org/10.1016/j.jhydrol.2014.09.027.

LIVADA, I.; ASSIMAKOPOULOS, V. D. Spatial and temporal analysis of drought in Greece using the Standardized Precipitation Index (SPI). Theoretical and Applied Climatology, v. 89, p. 143-153, 2007. http://dx.doi.org/;10.1007/s00704-005-0227-z.

LLOYD-HUGES, B.; SAUNDERS, M. A drought climatology for Europe. International Journal of Climatology, v. 22, p. 1571-1592, 2002. http://dx.doi.org/10.1002/joc.846

MCKEE, T. B.; DOESKEN, N. J.; KLEIST, J. The relationship of drought frequency and duration of time scales. Eighth Conference on Applied Climatology. American Meteorological Society, p. 179-186, 1993.

MISHRA, A. K.; SINGH, V. P. A review of drought concepts. Journal of Hydrology, v. 391, p. 202-216, 2010. http://dx.doi.org/10.1016/j.jhydrol.2010.07.012.

SANTOS, J. F.; PULIDO-CALVO, I.; PORTELA, M. M. Spatial and temporal variability of droughts in Portugal. Water Resources Research, v. 46, 2010. http://dx.doi.org/10,1029/2009WR008071

SIRDAS, S.; SEN, Z. Spatio-temporal drought analysis in the Trakya region, Turkey.

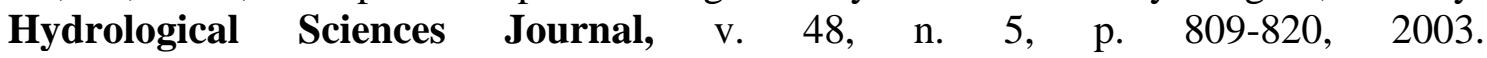
http://dx.doi.org/10.1623/hysj.48.5.809.51458

SONMEZ, F.; KOMUSCU, A.; ERKAN, A.; TURGU, E. An analysis of spatial and temporal dimension of drought vulnerability in turkey using the standardized precipitation index. Natural Hazards, v. 35, p. 243-264, 2005.

TEIXEIRA, C. F. A.; DAMÉ, R. C. F.; BACELAR, L. C. S.; SILVA, G. M.; COUTO, R. S. Intensidade da seca utilizando índices de precipitação. Revista Ambiente e Água, v. 8, n. 3, 2013. http://dx.doi.org/10.4136/ambi-agua.1245

UNITED NATIONS CONVENTION TO COMBAT DROUGHT AND DESERTIFICATION - UNCCD. United Nations Convention to Combat Drought and Desertification in Countries Experiencing Serious Droughts and/or Desertification. Particularly in Africa. Paris, 1994.

VICENTE-SERRANO, S. Spatial and temporal analysis of droughts in the Iberian Peninsula (1910-2000). Hydrological Sciences Journal, v. 51, p. 83-97, 2006. http://dx.doi.org/10.1623/hysj.51.1.83

WILHITE, D. A.; SVOBODA, M. D.; HAYES, M. J. Understanding the complex impacts of drought: A key to improving drought mitigation and preparedness. Water Resources Management, v. 21, n. 5, p. 763-774, 2007. http://dx.doi.org/10.1007/s11269-006-90765

WORLD METEOROLOGICAL ORGANIZATION - WMO. Report on Drought and Countries Affected by Drought During 1974-1985. Geneva, 1986. 118p. 
ZHAI, J.; SU, B.; KRYSANOVA, V.; VETTER, T.; GAO, C.; JIANG, T. Spatial variation and trends in PDSI and SPI indices and their relation to streamflow in 10 large regions of $\begin{array}{lllllll}\text { China. Journal of Climate, v. 23, p. 649-663, } 2010 . & \end{array}$ http://dx.doi.org/10.1175/2009JCLI2968.1 


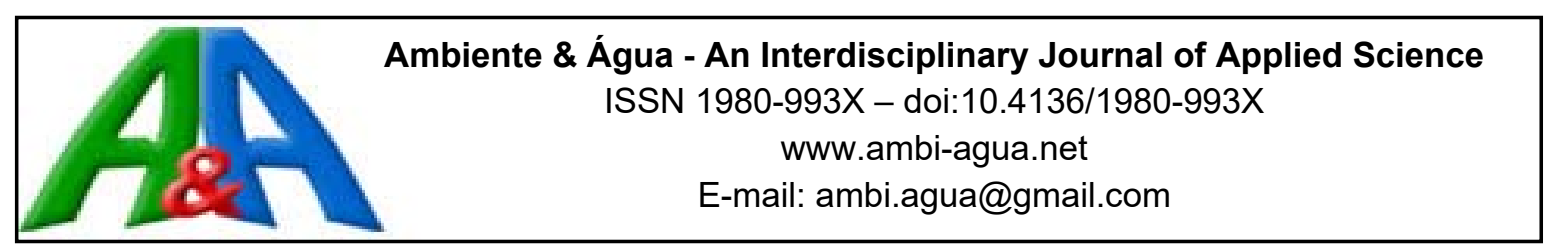

\title{
Avaliação do uso de extratos vegetais para controle da hemoncose em ovinos naturalmente infectados
}

doi:10.4136/ambi-agua.2020

Received: 3 Oct. 2016; Accepted: 25 Jan. 2017

\author{
Matheus Diniz Gonçalves Coêlho ${ }^{1 *}$; Thalyta Baldim Xavier ${ }^{1}$; \\ Jaqueline Fabiana da Costa ${ }^{1}$; Lucas Tobias Rodrigues Maciel ${ }^{1}$; \\ Lilian Saito Ormachea Bozo ${ }^{1}$; Francine Alves da Silva Coêlho ${ }^{2}$; \\ Gokithi Akisue ${ }^{1}$ \\ ${ }^{\mathbf{1}}$ Fundação Universitária Vida Cristã (FUNVIC), Pindamonhangaba, SP, Brasil \\ Laboratório de Parasitologia e Malacologia (LAPAM) \\ ${ }^{2}$ Universidade de Taubaté (UNITAU), Taubaté, SP, Brasil \\ Laboratório de Parasitologia \\ *Autor correspondente: e-mail: profmatheuscoelho@gmail.com, \\ thaly-ta17@hotmail.com, jaqueline_fabiana@yahoo.com.br, \\ ltrmaciel@gmail.com, liliaan.saito@gmail.com, \\ francine.ascoelho@gmail.com, akisuegokithi@gmail.com
}

\section{RESUMO}

A ovinocultura no Brasil tem sido considerada uma importante prática econômica. Entretanto, há alguns problemas relacionados com esta prática, dentre os quais se destaca a hemoncose. Haemonchus contortus é tido como o principal helminto parasita de ovelhas, considerando-se o fato de ser o único hematófago direto, podendo causar anemia, perda de peso, edema submandibular, baixa qualidade da carne e da lã e óbito de uma grande porcentagem do rebanho. Atualmente, opta-se pelo tratamento alopático para controle da hemoncose, porém, devido ao uso repetitivo, observa-se desenvolvimento crescente de resistência. Neste sentido, a fitoterapia tem se destacado como uma alternativa promissora. Sendo assim, no presente trabalho objetivou-se avaliar a atividade anti-parasitária de três extratos vegetais: extratos hidroalcoólicos obtidos por percolação de Lepidium didymum e Momordica charantia, e extrato aquoso de Tagetes minuta. Animais da raça santa Inês foram triados para identificação de espécimes parasitados e separados em quatro grupos com seis animais cada, sendo um grupo controle sem tratamento, e os demais tratados com $200 \mathrm{mg} / \mathrm{dia}(5 \mathrm{mg} / \mathrm{kg}$ de peso corpóreo) com os três extratos, durante 5 dias. Após o tratamento foi realizada determinação de OPG (ovos por grama de fezes) pelo método MacMaster com modificação. Observou-se que o os animais dos grupos tratados com os extratos de L. didymum e $M$. charantia apresentaram redução significativa $(\mathrm{p}<0,05)$ do número de OPG observado, portanto, esses extratos podem ser úteis no tratamento da hemoncose ovina.

Palavras-chave: Haemonchus contortus, medicamentos fitoterápicos, ovinos.

\section{Evaluation of plant extracts to control haemonchosis in naturally infected sheep}

\section{ABSTRACT}

Sheep husbandry is considered an important economic practice in Brazil. However, there are problems associated with this practice. Haemonchosis stands out among these, and is known 
among sheep farmers for being a difficult disease to treat. Haemonchus contortus is considered the main helminth parasite of sheep, due to the fact that it is the only direct blood-sucking parasite, which can cause anemia, weight loss, submandibular swelling (mumps), low quality of meat and wool, and death of a large percentage of the herd. Currently, the predominant treatment is allopathic medicines, but resistance is increasing due to their repetitive use. In this sense, herbal medicine has emerged as a promising alternative. This study therefore aimed to evaluate the in vivo anti-parasitic activity of three plant extracts: hydroalcoholic extracts obtained by the percolation of Lepidium didymum and Momordica charantia and aqueous extract of Tagetes minuta. Animals of the Santa Inês breed were screened to identify parasitized specimens and separated into four groups of six animals each. One was used as an untreated control group, and the others were treated with $200 \mathrm{mg} /$ day $(5 \mathrm{mg} / \mathrm{kg}$ body weight) of the three extracts for 5 days. After treatment, EPG (eggs per gram of feces) was determined by the Mac Master method with modification. The animals in the groups treated with extracts of $L$. didymum and $M$. charantia showed a significant reduction $(\mathrm{p}<0.05)$ of EPG. These extracts may therefore be useful in the treatment of sheep haemonchosis.

Keywords: Haemonchus contortus, herbal medicines, sheep.

\section{INTRODUÇÃO}

O Brasil, com sua enorme extensão territorial e clima favorável à espécie ovina, apresenta altíssimo potencial para tornar-se importante produtor mundial de ovinos (Poli et al., 2008). A ovinocultura consiste em importante prática econômica no setor agropecuário devido à facilidade na criação deste tipo de rebanho, porém, um dos principais problemas encontrados nessa prática, e que vem chamando atenção dos produtores, são as parasitoses gastrintestinais (Costa et al., 2009).

Tais enfermidades limitam consideravelmente o aproveitamento econômico destes animais, destacando-se, neste sentido, a hemoncose, já que se trata de uma doença de difícil tratamento, cujo agente etiológico, Haemonchus contortus, desenvolveu, no decorrer dos anos, forte resistência aos antiparasitários hoje disponíveis comercialmente, trazendo como consequências impactos negativos para economia, com elevada mortalidade e perda de produtividade (Costa et al., 2009).

No Brasil a maioria dos rebanhos é criada de forma extensiva, estando exposta à infecção por helmintos. Este fato se agrava quando estes animais são criados em áreas que comportam altas lotações, o que acaba por gerar um aumento na contaminação ambiental, com elevada densidade populacional do parasito, e elevada ocorrência do estágio de vida livre do mesmo, que é responsável pela infecção do rebanho (Amarante e Sales, 2007).

$H$. contortus, a principal espécie de parasito de ovinos no Brasil, pertence ao filo Nemathelminthes, Classe Nematoda, Ordem Strongylida, Família Trichostrongylidae e Gênero Haemonchus, sendo um parasita hematófago do abomaso (Melo et al., 2003). A hemoncose é uma doença cujos sintomas apresentados pelos animais que possuem alta carga parasitaria são: edema submandibular, conhecido popularmente como papeira, queda do volume globular, anemia e perda de peso, apresentando comumente um índice de letalidade significativo (Endo et al., 2014).

Decorrente do insuficiente repasse de tecnologia, ou de informações quanto à utilização correta das drogas antiparasitárias em ovinos, observou-se grande diminuição da eficácia desses produtos, inclusive, com o aparecimento de cepas resistentes a vários grupos químicos disponíveis no mercado, destacando-se neste sentido a busca por novas estratégias terapêuticas, dentre as quais a fitoterápica (Molento et al., 2004). 
A fitoterapia é o tratamento de enfermidades através de vegetais frescos, drogas vegetais, ou extratos vegetais, sendo considerado um tratamento atraente, em que o impacto ambiental e os resíduos podem ser minimizados, possibilitando redução de custo (Klein et al., 2009; Chagas, 2008). Além disso, a fitoterapia é uma ferramenta que permite aumentar a variedade de produtos a serem utilizados; ofertar opções terapêuticas de medicamentos equivalentes, também registrados, talvez mais baratos e com ação mais adequada e, possivelmente, com indicações terapêuticas complementares às medicações existentes (Pinto et al., 2002).

A ausência de um tratamento antiparasitário alopático totalmente eficiente frente a H. contortus maximiza a demanda pelo desenvolvimento de novos medicamentos capazes de controlar essa parasitose, e destaca a busca por tratamentos, dentro os quais o uso de fitoterápicos que possam ser aplicados à parasitologia veterinária, já que muitas plantas são tradicionalmente conhecidas como possuidoras de atividade anti-helmíntica e, em paralelo, são passíveis de causar menos impactos ambientais e de induzir resistência nos parasitos (Molento et al., 2004).

Sendo assim, no presente trabalho objetivou-se avaliar in vivo a atividade anti-parasitária, frente a Haemonchus contortus, de três extratos vegetais, a saber: extratos hidroalcoólicos obtidos por percolação de Lepidium didymum e Momordica charantia e extrato fluido alcoólico de Tagetes minuta, em ovinos naturalmente infectados.

\section{MATERIAL E MÉTODOS}

As espécies vegetais oriundas da região do Vale do Paraíba foram identificadas botanicamente, sendo as exsicatas depositadas no herbário SPF (Universidade de São Paulo), para confirmação da identidade botânica, cujos respectivos números estão citados a seguir, após o nome científico de cada espécie avaliada: L. didymum (exsicata G. akisue 064), M. charantia (exsicata G. akisue 063) e T. minuta (G. Akisue 033). Para obtenção dos extratos no Laboratório de Farmacognosia e plantas medicinais da FUNVIC - Faculdade de Pindamonhangaba (LAFAPLAM), as partes aéreas (folhas, flores e talos) de cada vegetal foram processadas, secadas em estufa a $45^{\circ} \mathrm{C}$ e pulverizadas em moinho de café elétrico (Cuisinart ${ }^{\circledR}$ ). Foram avaliados extratos etanólicos obtidos por percolação de L. didymum e M. charantia, e T. minuta, obtido pelo processo "C", segundo a Farmacopéia Brasileira 2a edição.

Para obtenção dos extratos por percolação, empregou-se etanol a 70\% v/v como líquido extrator.

O processo foi conduzido até o momento em que o líquido percolado não apresentava coloração, sendo o mesmo concentrado por meio de evaporação do solvente em aparelho rotavapor (Silva et al., 2013).

Para obtenção do extrato fluido alcoólico, foi utilizado o processo "C" de percolação fracionada segundo a Farmacopéia Brasileira $2^{\circ}$ edição (Furtado et al., 2013), onde foi utilizado $300 \mathrm{~g}$ da droga, conforme segue:

Para a $1^{\mathrm{a}}$ extração, pesou-se $150 \mathrm{~g}$ da droga, umedeceu-se uniformemente com o líquido extrator (álcool $70 \% \mathrm{v} / \mathrm{v}$ ), em seguida o pó umedecido foi transferido para percolador adequado, onde a droga foi coberta completamente pelo líquido extrator e deixou-se macerar. Foi separada uma fração de $60 \mathrm{~mL}$ e recolhidas mais cinco frações de $70 \mathrm{~mL}$.

Para a $2^{\mathrm{a}}$ extração, pesou-se $90 \mathrm{~g}$ da droga, umedeceu-se com a $1^{\mathrm{a}}$ porção de $70 \mathrm{~mL}$ do percolato obtido a partir da $1^{\text {a }}$ extração, e, em seguida, o pó umedecido foi transferido para o percolador. Como líquido extrator, foram utilizadas as porções restantes do percolato obtido na $1^{a}$ extração (quatro frações restantes de $70 \mathrm{~mL}$ ) e usadas na ordem em que foram recolhidas. Após término da $2^{\text {a }}$ extração, foi separada uma fração de $90 \mathrm{~mL}$ e recolhidas cinco frações de $60 \mathrm{~mL}$. 
Para a $3^{\mathrm{a}}$ extração, pesou-se $60 \mathrm{~g}$ da droga, umedeceu-se com a $1^{\mathrm{a}}$ porção de $60 \mathrm{~mL}$ do percolato obtido a partir da $2^{\mathrm{a}}$ extração, e, em seguida, o pó umedecido foi transferido para o percolador. Como líquido extrator, foram utilizadas as porções restantes do percolato obtido na $2^{\mathrm{a}}$ extração (quatro frações restantes de $60 \mathrm{~mL}$ ) e usadas na ordem em que foram recolhidas. Após término da $3^{\mathrm{a}}$ extração, foi separada uma fração de $150 \mathrm{~mL}$.

Ao final foram misturadas as três frações dos percolatos obtidas das três extrações $(60 \mathrm{~mL}+90 \mathrm{~mL}+150 \mathrm{~mL})$, totalizando $300 \mathrm{~mL}$ de extrato fluido.

\subsection{Animais}

Os animais participantes do experimento foram oriundos de uma propriedade no município de Pindamonhangaba- SP. O rebanho ovino da propriedade é de aproximadamente 1.000 cabeças da raça Santa Inês, criado em sistema integrado com bovinos e alimentado exclusivamente através do pastoreio de campo nativo, apresentando, de acordo com o criador, altos índices de verminose historicamente, só sendo possível a manutenção deste sistema de produção através do estabelecimento de um programa de controle de verminose pelo veterinário responsável pela propriedade. Assim, estabeleceu-se de comum acordo com o proprietário e este profissional, o não tratamento do lote de animais alvo do experimento durante um período mínimo de 30 dias (na última medicação anti-helmíntica foi aplicado produto a base de Closantel), e a permanência destes animais em um mesmo piquete.

\subsection{Seleção dos animais}

Para a seleção dos animais participantes do experimento, amostras fecais foram coletadas diretamente da ampola retal de 50 ovinos, fêmeas, com peso vivo médio de $40 \mathrm{~kg}$, entre seis e sete meses de idade. As amostras coletadas foram armazenadas em sacos plásticos identificados, sendo conduzidas em caixa térmica para o Laboratório de Parasitologia e Malacologia (LAPAM) da Fundação Universitária Vida Cristã/Faculdade de Pindamonhangaba (FUNVIC) onde foi realizado o Método de Gordon \& Whitlock modificado com auxílio de câmara de McMaster, para determinação de OPG, de modo a selecionar, para compor os grupos experimentais, apenas os 24 animais que apresentaram um número de ovos por grama (OPG) igual ou superior a 500 .

Brevemente, um grama ( $1 \mathrm{~g})$ de fezes foi misturado a 14 mililitros $(14 \mathrm{~mL})$ de solução de $\mathrm{NaCl}(\mathrm{d}=1,2)$ e um volume de $150 \mu \mathrm{L}$ da suspensão obtida foi transferido para uma câmara MacMaster, para determinação de OPG.

\subsection{Delineamento experimental}

Os animais foram identificados através de brincos numerados, e amostras fecais individuais foram coletadas um dia antes do início do experimento. Os animais foram divididos em 4 grupos de 6 animais através de sorteio (amostragem aleatória simples), sendo um grupo controle sem tratamento, e os demais tratados com $200 \mathrm{mg} / \mathrm{dia}(5 \mathrm{mg} / \mathrm{kg}$ de peso corpóreo) dos extratos supracitados, diluídos em $100 \mathrm{~mL}$ de etanol a $80 \%$, durante 5 dias, sendo:

Grupo 1: 6 ovinos sem tratamento (grupo controle).

Grupo 2: 6 ovinos, os quais receberam extrato hidroalcoólico obtido por percolação de L. didymum.

Grupo 3: 6 ovinos, aos quais foi administrado extrato hidroalcoólico obtido por percolação M. charantia.

Grupo 4: 6 ovinos, ao quais foi administrado extrato fluido alcoólico obtido por percolação de T. minuta. 
Depois de estabelecida a terapêutica, amostras fecais foram coletadas após 10 dias e foi realizada determinação de OPG. Todos os animais também foram acompanhados clinicamente, no que diz respeito ao bem-estar geral.

O percentual de redução de OPG foi determinado empregando-se os valores das médias de OPG do primeiro dia antes de iniciado o tratamento (dia zero) e as médias das respectivas amostras coletadas, utilizando-se a seguinte fórmula (Equação 1), descrita por Jacobs et al. (1994).

$\%$ de redução de $O P G=\frac{\text { (média de OPG (dia zero) }- \text { média de OPG }(\text { dia de interesse })}{(\text { média de OPG }(\text { dia zero })} \times 100$

Os resultados obtidos foram avaliados estatisticamente utilizando teste de ANOVA ou Kruskal-Wallis, de acordo com a normalidade das médias obtidas, seguidos do teste de Tukey ou o teste de Student-Newman-Keuls, para verificar diferenças entre as médias. De forma complementar, foi realizado o teste do Qui-quadrado para verificar diferenças entre as médias de redução de OPG de cada grupo em relação à observada no grupo controle.

\section{RESULTADOS E DISCUSSÃO}

Observou-se que após 10 dias (dia 15) do término do tratamento, todos os extratos vegetais apresentaram atividade antiparasitária, já que a média do número de OPG foi significativamente inferior $(\mathrm{p}<0,05)$, quando comparada à observada um dia antes do início do tratamento (Tabela 1).

O extrato vegetal que se apresentou mais promissor no controle da hemoncose em ovinos foi o obtido a partir de $M$. charantia, que induziu uma redução de 79,5\% de OPG, sendo este valor significativamente superior $(\mathrm{p}<0,0001 \%)$ aos observados no grupo controle, e aos resultados observados nos grupos tratados com os dois outros extratos vegetais avaliados (Tabela 1).

Tabela 1. Média de OPG e redução de OPG observada em ovinos naturalmente infectados e tratados com extratos hidroalcoólicos obtidos de L. didymum e M. charantia e extrato aquoso de $T$. minuta, na dose de $1 \mathrm{~g} /$ dia durante 5 dias consecutivos.

\begin{tabular}{lccc}
\hline Tratamento & $\begin{array}{c}\text { Dias de } \\
\text { tratamento }\end{array}$ & $\begin{array}{c}\text { Média } \\
\text { OPG }\end{array}$ & $\begin{array}{c}\text { Redução } \\
\text { OPG 15 }\end{array}$ \\
\hline \multirow{3}{*}{ L. didymum } & 0 & 2775 & \\
& 7 & 1133 & 63,7 \\
T. minuta & 15 & 1008 & \\
& 0 & 3916 & \\
& 7 & 2525 & 51,5 \\
M. charantia & 15 & 1900 & \\
& 0 & 3700 & \\
& 7 & 1200 & 79,5 \\
Controle & 15 & 758 & \\
& 0 & 1200 & \\
& 7 & 1700 & 46,2 \\
\hline
\end{tabular}

Nota: $O P G=$ ovos por grama de fezes. 
A atividade antiparasitária de $M$. charantia, in vivo observada no presente trabalho, foi superior aos resultados apresentados por Vieira et al. (1999), segundo os quais a administração de droga vegetal de $M$. charantia na concentração de $1 \mathrm{~g} / \mathrm{kg}$ de peso corpóreo de ovinos durante quatro dias seguidos, induziu a uma redução de 36\% de OPG e 17\% da carga parasitária de Haemonchus contortus. Embora no presente trabalho não tenha sido avaliada a redução de carga parasitária por meio de quantificação de vermes adultos intestinais, é possível destacar a relevância dos resultados aqui apresentados, tendo em vista a redução de OPG que foi observada.

Cabe ressaltar que Vieira et al. (1999) avaliaram a eficácia da droga vegetal, que consiste no vegetal seco e pulverizado, não tendo realizado nenhum processo de extração que pudesse concentrar princípios ativos. Em contrapartida, no presente trabalho, procedeu-se uma extração com uso de solvente (etanol) e posterior evaporação em aparelho rotavapor, que permitiu uma concentração de princípio ativo, e consequente aumento de eficácia na redução de OPG.

$\mathrm{O}$ delineamento de experimentos futuros utilizando solventes com polaridades crescentes poderá, possivelmente, permitir o isolamento de grupos mais restritos de substâncias ativas, o que pode ser promissor no sentido de maximizar a atividade antiparasitária traduzida por uma redução mais significativa de OPG.

Em uma revisão bibliográfica, Camurça-Vasconcelos et al. (2005) reuniram informações sobre estudos com plantas medicinais para uso como antiparasitários em pequenos ruminantes, e concluíram que, dentre os vegetais avaliados por diversos autores, $M$. charantia foi o mais promissor para o controle da hemoncose.

No presente estudo, foi utilizada uma solução na concentração de $5 \mathrm{mg} / \mathrm{kg}$ de peso corpóreo para cada animal, sendo esta preparada a partir do extrato bruto de $M$. charantia, que foi obtido por percolação, e posterior concentração em rotavapor utilizando álcool $80^{\circ}$ como solvente extrator. Embora a obtenção do extrato bruto seja consideravelmente mais laboriosa, o uso do mesmo representa uma vantagem quando comparado ao uso do vegetal in natura.

É possível que o processo de extração utilizado facilite a absorção de diversos princípios ativos ou componentes químicos que o processo de digestão do vegetal in natura possivelmente não permitiria que ocorresse.

A redução de OPG induzida quando do uso do extrato de $M$. charantia também foi superior aos resultados obtidos por Brito-Júnior et al. (2011), que obtiveram uma redução de $40 \%$ de OPG ao avaliarem a eficácia do extrato alcoólico deste vegetal na concentração de $5,85 \mathrm{mg} / \mathrm{kg}$ de peso corpóreo, que foi administrado por 3 dias consecutivos em caprinos naturalmente infectados.

Por outro lado, os dados apresentados foram semelhantes aos resultados obtidos por Almeida et al. (2007) que, ao avaliarem a atividade de plantas medicinais frente a $H$. contortus em caprinos da região do Semiárido Paraibano, observaram uma redução de OPG de $82 \%$ 60 dias após término do tratamento, porém utilizando as folhas secas desse vegetal na dose de $4,5 \mathrm{~g} / \mathrm{kg}$ de peso corpóreo, sendo administrados por 3 dias consecutivos.

As possíveis diferenças de eficácia na redução de OPG supracitadas podem ser decorrentes de fatores relacionados ao esquema terapêutico adotado ou das características climáticas e geográficas, como por exemplo, na qualidade e quantidade de nutrientes do solo, que podem influenciar na maior ou menor concentração de determinados princípios ativos, e que, por consequência, podem influenciar na atividade biológica das mesmas (Furtado et al., 2013).

No grupo tratado com extrato de $L$. didymum foi observada redução de $63,66 \%$ na média de OPG, sendo este valor significativamente superior $(\mathrm{p}<0,0001 \%)$ ao observado no grupo controle. São escassas as pesquisas que avaliaram atividade biológica deste vegetal. Stolz et al. (2014) realizaram um estudo etnobotânico visando determinar o uso popular de vegetais no Rio Grande do Sul. Estes autores, após extensa revisão da literatura, afirmaram que são poucos os estudos clínicos a respeito de L. didymum e as suas propriedades comprovadas estão limitadas

Rev. Ambient. Água vol. 12 n. 2 Taubaté - Mar. / Apr. 2017 
ao uso no reumatismo, como antipirético, anti-inflamatório e hepatoprotetor, estando estas propriedades possivelmente relacionadas com a presença de flavonoides, saponinas e taninos.

Os resultados obtidos no presente trabalho trazem à tona mais um potencial uso deste vegetal, e norteia futuros estudos que visem pormenorizar os mecanismos através dos quais L. didymum apresentou eficácia antiparasitária frente a $H$. contortus.

Com relação a T. minuta, observou-se que o extrato obtido a partir desse vegetal induziu a uma redução de OPG de 51,5\% (Tabela 1). Até onde se estendeu a busca por artigos científicos realizada no presente estudo, não há relatos de atividade antiparasitária de T. minuta frente a H. contortus in vivo. Entretanto esse vegetal tem demonstrado atividade biológica frente a diversos patógenos in vitro, dentre os quais microrganismos (Souza et al., 2000), carrapatos (Furtado et al., 2013), fito nematóides (Moraes et al., 2006) e até mesmo H. contortus (Macedo, 2012).

Macedo (2012) demonstrou que o decocto obtido da parte aérea de T. minuta foi capaz de induzir uma inibição de $50 \%$ de eclosão de larvas de $H$. contortus na concentração de $0,6 \mathrm{mg} / \mathrm{mL}$, e uma inibição de $50 \%$ do desembainhamento larvar na concentração de 620 $\mu \mathrm{g} / \mathrm{mL}$, e destacaram a possibilidade de uso deste para o controle da hemoncose, desde que realizados estudos in vivo.

No presente trabalho observa-se que, embora a atividade in vitro de $T$. minuta frente a $H$. contortus tenha sido anteriormente demonstrada, o extrato aquoso deste vegetal quando administrado na dose de $5 \mathrm{mg} / \mathrm{kg}$ de peso corpóreo de animal durante cinco dias não foi capaz de promover atividade antiparasitária significativa.

\section{CONCLUSÃO}

Os resultados obtidos permitiram concluir que os extratos hidroalcoólicos obtidos a partir de folhas de Momordica Charantia e Lepidium didymum são potencialmente úteis para o tratamento da hemoncose ovina, já que apresentaram atividade antiparasitária na dose de $5 \mathrm{mg} / \mathrm{kg}$ de peso corpóreo durante cinco dias, o mesmo não ocorrendo com o extrato aquoso de T. minuta. Destacado-se, porém, a importância de novos experimentos que visem avaliar essa propriedade, utilizando novos solventes para extração, e administração de doses mais elevadas.

\section{REFERÊNCIAS}

ALMEIDA, W. V. F.; SILVA, R. C. L. M.; FARIAS, B. E.; ATHAYDE, R. C. A.; SILVA, W. W. Avaliação de plantas medicinais em caprinos da região do semiárido paraibanos naturalmente infectados por nematoides gastrintestinais. Revista Caatinga, v. 20, n. 3, p. 1-7, 2007. Disponível em: http://www.redalyc.org/articulo.oa?id=237117565001

AMARANTE, A. F. T.; SALES, R. O. Controle de endoparasitoses dos ovinos: uma revisão. Revista Brasileira de Higiene e Sanidade Animal, v. 1, n. 2, p. 14-36, 2004. http://dx.doi.org/10.5935/1981-2965.20070007

BRITO-JUNIOR, L.; SILVA, R. C. L. M.; LIMA, H. F.; ATHAYDE, R. C. A.; SILVA, W. W.; RODRIGUES, G. O. Estudo comparativo da ação anti-helmínticas da batata de purga (Operculina hamiltonii) e do melão de são caetano (Mormodica charantia) em caprino (Capra hircus) naturalmente infectado. Ciência e Agrotecnologia, v. 35, n. 4, p. 797802, 2011. http://dx.doi.org/10.1590/S1413-70542011000400020

CHAGAS, A. C. S. Fitoterapia como alternativa no controle de verminose em caprinos e ovinos. São Carlos: Instituto de Zootecnia/Embrapa Pecuária Sudeste, 2008. p. 75-79. 
CAMURÇA-VASCONCELOS, A. L. F.; MORAIS, S. M.; SANTOS, L. F. L.; ROCHA, M. F. G.; BEVILAQUA, C. M. L. Validação de plantas medicinais com atividade antihelmíntica. Revista Brasileira de Plantas Medicinais, v.7, n. 3, p. 97-106, 2005.

COSTA, V. M. M.; SIMÕES, S. V. D.; RIET-CORREA, F. Doenças parasitárias em ruminantes no semi-árido brasileiro. Pesquisa Veterinária Brasileira, v. 29, n. 7, p. 563-568, 2009. http://dx.doi.org/10.1590/S0100-736X2009000700011

ENDO, V. T.; OLIVEIRA, T. C.; CABRAL, A. P. M.; SAKAMOTO, C. A. M.; FERRARO, G. C.; PEREIRA, V. et al. Prevalência dos helmintos Haemonchus contortus e Oesophagostomum columbianum em pequenos ruminantes atendidos no setor de anatomia patológica - UEM. Revista de Ciência Veterinária e Saúde Pública, v. 1, n. 2, p. 112-118, 2014. http://dx.doi.org/10.4025/revcivet.v1i2.25397

FURTADO, F. N.; SILVA, V. A. R.; PEREIRA, J. R.; AKISUE, G. A.; CÔELHO, F. A. S.; COÊLHO, M. D. G. Avaliação "in vitro" do potencial acaricida do óleo essencial de Tagetes minuta frente a Riphicephalus (Boophilus) microplus (Canestrini, 1887). Revista Biociências, v. 19, n. 1, p. 104-110, 2013.

JACOBS, D. E.; ARAKAWA, A.; COURTNEY, C. H.; GEMMELL, M. A.; MCCALL, J. W.; MYERS, G. H. et al. World Association for the Advancement of Veterinary Parasitology (W.A.A.V.P) guidelines for evaluating the efficacy of Anthelmintics for dogs and cats. Veterinary Parasitology, v. 52, p. 179-202, 1994. http://dx.doi.org/10.1016/03044017(94)90110-4

KLEIN, T.; LONGHINI, R.; BRUSCHI, M. L.; MELLO, J. C. P. Fitoterápicos: um mercado promissor. Revista Brasileira de Ciências Farmacêuticas Básica e Aplicada, v. 30, n. 3, p. 241-248, 2009.

MACEDO, F. T. I. Atividade anti-helmíntica de óleos essenciais de plantas do nordeste brasileiro. 2012. Tese (Doutorado em Ciências Veterinárias) - Faculdade de Veterinária, Universidade Estadual do Ceará, Fortaleza, 2012.

MELO, A. C. F. L.; REIS, I. F.; BEVILAQUA, C. M. L.; VIEIRA, L. S.; ECHEVARRIA, F. A. M.; MELO, L. M. Nematódeos resistentes a anti-helmíntico em rebanhos de ovinos e caprinos do estado do Ceará, Brasil. Ciência Rural, v. 33, n. 2, p. 339-344, 2003. http://dx.doi.org/10.1590/S0103-84782003000200024

MOLENTO, M. B.; TASCA, C.; GALLO, A.; FERREIRA, M.; BONONI, R.; STECCA, E. Método famacha como parâmetro clínico individual de infecção por Haemonchus contortus em pequenos ruminantes. Ciência Rural, v. 34, n. 4, p. 1139-1145, 2004. http://dx.doi.org/10.1590/S0103-84782004000400027

MORAES, S. R. G.; CAMPOS, V. P.; POZZA, E. A.; FONTANETTI, A.; CARVALHO, G. J.; MAXIMINIANO, C. Influência de leguminosas no controle de fitonematóides no cultivo orgânico de alface americana e de repolho. Fitopatologia Brasileira, v. 31, n. 2, p. 188-191, 2006. http://dx.doi.org/10.1590/S0100-41582006000200011

PINTO, A. C.; SILVA, D. H. S.; BOLZANI, V. S.; LOPES, N. P.; EPIFANIO, R. A. Produtos Naturais: Atualidade, Desafios e Perspectivas. Química Nova, v. 25, n. 1, p. 45-61, 2002. http://dx.doi.org/10.1590/S0100-40422002000800009 
POLI, C. H. E. C.; MONTEIRO, A. L. G.; BARROS, C. S.; MORAES, A.; FERNANDES M. A. M.; PIAZZETTA, H. V. L. Produção de ovinos de corte em quatro sistemas de produção. Revista Brasileira de Zootecnia, v. 37, n. 4, p. 666-673, 2008. http://dx.doi.org/10.1590/S1516-35982008000400012

SILVA, R. C. S; ALMEIDA, J. C. R.; ALMEIDA, A. A. S.; AKISUE, G.; COÊLHO, M. D. G. Avaliação da toxicidade dos extratos do Araribá (Centrolobium tomentosum) com utilização do ensaio com Artemia salina. Revista Ambiente \& Água, v. 8, supl., p. 158167, 2013. http://dx.doi.org/10.4136/ambi-agua.1374

SOUZA, S. A. C.; MARCHIONATTI, C. A.; WIEST, M. J. Atividade antimicrobiana de Tagetes minuta L.- Compositae (Chinchilho) frente à bactérias gram-positivas e gram negativas. Brazilian Journal of Veterinary Research Animal Science, v. 37, n. 6, p. 429-433, 2000. http://dx.doi.org/10.1590/S1413-95962000000600001

STOLZ, D. E.; MULLER, G. L.; RODRIGUES, T. M.; BAUMHARDT, E.; RITTER, R. M.; RATES, K. M. S. Survey of plants popularly used for pain relief in Rio Grande do Sul, Southern Brazil. Revista Brasileira de Farmacognosia, v. 24, p. 185-196, 2014. http://dx.doi.org/10.1016/j.bjp.2014.03.007

VIEIRA, L. S.; CAVALCANTE, A. C. R.; PEREIRA, M. F.; DANTAS, L. B.; XIMENES, L. J. F. Evaluation of anthelmintic efficacy of plants available in Ceara State, Northeast Brazil, for the control of goat gastrointestinal nematodes. Revue Medicine Veterinaire, v. 150, n. 5, p. 447-452, 1999. http://dx.doi.org/10.1590/S1516-05722009000300016 


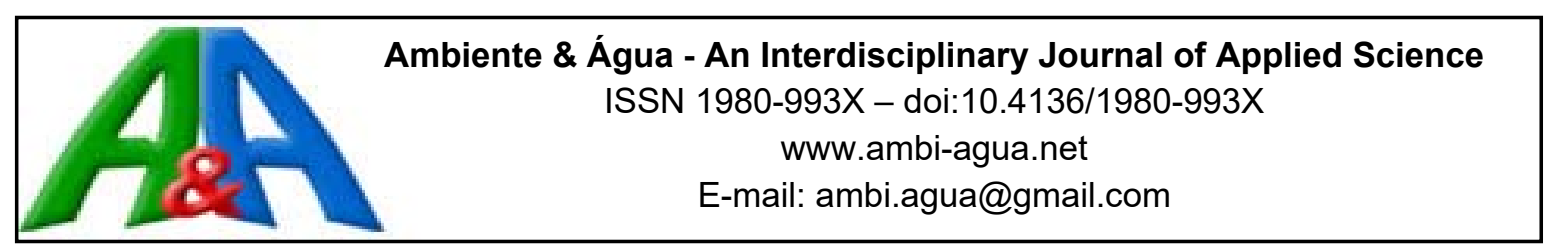

\title{
Variáveis intervenientes na existência de comitês de bacias hidrográficas no Brasil ${ }^{1}$
}

\author{
doi:10.4136/ambi-agua.1828
}

Received: 21 Dec. 2015; Accepted: 26 Jan. 2017

\author{
Alexandre André Feil ${ }^{1 *}$; Vírgilio José Strasburg²; Fernando Rosado Spilki ${ }^{3}$ \\ ${ }^{1}$ Centro Universitário Univates, Lajeado, RS, Brasil \\ Departamento do Centro de Gestão Organizacional \\ ${ }^{2}$ Universidade Federal do Rio Grande do Sul (UFRGS), Porto Alegre, RS, Brasil \\ Departamento de nutrição \\ ${ }^{3}$ Universidade FEEVALE, Novo Hamburgo, RS, Brasil \\ *Autor correspondente: e-mail: alexandre.feil1@gmail.com, \\ vjs.nut@terra.com.br, fernandors@feevale.br
}

\section{RESUMO}

A gestão de bacias hidrográficas por meio de comitês é essencial na mediação entre a oferta e a demanda de água para consumo doméstico, industrial, agrícola, lazer, entre outros usos da água. Uma gestão exitosa pode ocorrer por meio da identificação e compreensão das características socioeconômicas, demográficas, entre outras, e assim formatar as práticas adotadas pelos comitês. Neste contexto, o escopo central deste estudo consistiu em analisar as variáveis intervenientes relacionadas aos aspectos geográficos, demográficos, socioeconômicos e a existência de comitê de bacias. A metodologia consiste na abordagem quantitativa, empregando-se uma pesquisa documental secundária e sua análise ocorreu por meio da aplicação da estatística descritiva e do teste de correlação de Pearson. Os principais resultados evidenciam que a explicação da existência de comitês de bacias hidrográficas relaciona-se com o tamanho da população que promove maior pressão à medida que aumenta. Além disso, evidenciou-se que a existência de comitês de bacias correlaciona-se com: crescimento econômico, quantidade de conflitos pelos múltiplos usos, coleta de esgotos e nível de degradação da qualidade ambiental. Conclui-se, portanto, que de fato existem variáveis intervenientes para a existência de comitês de bacias e que eles são necessários para a gestão eficiente destas variáveis.

Palavras-chave: demográficos, geográficos, Pearson test, recursos hídricos, saneamento, socioeconômicos.

\section{Intervening variables in the existence of watershed committees in Brazil}

\begin{abstract}
Watershed management through committees is essential in mediating between water supply and demand for domestic consumption, as well as industrial, agricultural, leisure, and other uses of water. Successful management can occur through the identification and

\footnotetext{
${ }^{1}$ Uma versão deste artigo foi publicada como resumo expandido no Seminário de Pós-Graduação, 2014 com o título: "Fatores que influenciam na consolidação de Comitês das Bacias Hidrográficas brasileiras". Novo Hamburgo RS: Feevale, 2014. v. 6. p. 175-80. ISSN 2358-4599
} 
comprehension of socioeconomic and demographic characteristics, among others, and therefore, these help shape the practices adopted by the committees. In this context, the central objective of this study was to analyse the intervening variables related to the geographic, demographic, socioeconomic aspects and the existence of a basin committee. The methodology consisted of a quantitative approach, using previously published documents and analysis applying descriptive statistics and the Pearson correlation test. The main results showed that the existence of a watershed committee is correlated with the size of the population, which promotes greater pressure as it increases. In addition, it was observed that the creation of committees is related to economic growth, number of conflicts for multiple uses, sewage collection and level of environmental quality degradation. We conclude, therefore, that in fact there are intervening variables for the existence of river basin committees and that the committees are necessary for the efficient management of those variables.

Keywords: geographic, demographic, Pearson test socioeconomic, sanitation, water resources.

\section{INTRODUÇÃO}

A gestão de Bacias Hidrográficas (BHs) por meio de Comitês de Bacias Hidrográficas (CBHs) é essencial na solução de conflitos, principalmente pela degradação e distribuição equitativa da água aos inúmeros interessados. A gestão dos recursos hídricos deve estar direcionada aos recursos naturais em função do crescimento de cada componente na $\mathrm{BH}$, pois, as participações públicas envolvidas nos $\mathrm{CBHs}$ possuem muitas perspectivas, interesses e alternativas de uso dos recursos hídricos em função da diversidade, renda, prioridades, localização, posições, entre outras (Sanchez-Roman et al., 2009).

As BHs compreendem uma área, na qual após a precipitação, a água escorre através de redes de córregos pelo processo natural, até desaguar em um rio, lago, represa ou oceano, que pode distanciar-se a alguns ou a milhares de hectares da área da superfície (Datta, 2015). O desenvolvimento de BHs compreende um instrumento para obtenção de melhorias na distribuição, resolução de conflitos de interesses, produtividade, preservação e conservação da água, onde a degradação de recursos, principalmente os hídricos compreendem um grave problema (Hope, 2007). Neste sentido, a gestão de BHs é um instrumento - em função da disponibilidade da base de dados e informações, clara definição dos diretos de uso, controles de impactos aos recursos hídricos e o processo de tomada de decisão - essencial na preservação da qualidade e quantidade, utilização racional e integrada dos recursos hídricos e pela influência de diversas variáveis intervenientes (Silva et al., 2008).

Os CBHs possuem como escopo a gestão destes recursos hídricos de uma unidade territorial denominada de $\mathrm{BH}$ por meio da participação pública. Esta participação pública em função da diversidade de membros agrega diversos benefícios, pois possuem diferentes relações com o meio ambiental, em especial, com a água (Hajkowicz, 2007; Perkins, 2011; Malheiros et al., 2013).

A participação pública abrange indivíduos ou grupos afetados positiva e negativamente pela disponibilidade de recursos hídricos cujos interesses influem ou sejam influenciados pelo projeto proposto, programa, plano ou política, as quais vinculam-se ao processo de tomada de decisão (André et al., 2006). Liu et al. (2008), Kaplowitz e Witter (2008) e Perkins (2011) salientam que a incorporação das comunidades locais no processo de planejamento melhora o sucesso da gestão dos recursos hídricos pelos CBHs. Os objetivos da participação pública na gestão dos CBHs, segundo André et al. (2006), compreendem: a) promover a justiça, equidade e colaboração; b) informar as medidas e suas consequências aos diversos stakeholders; c) promover a aprendizagem recíproca do público participante; e d) reduzir os impactos negativos e mitigar conflitos (Porto et al., 1999). Portanto, a forma organizada dessa participação ocorre mediante a estrutura dos CBHs. 
Os processos de participação na gestão de BHs por meio de CBHs possuem aspectos positivos e negativos (Korff et al., 2010). Estes autores destacam que os aspectos positivos se vinculam à legitimidade das tomadas de decisões pelas partes interessadas e mais adequadas em função da consideração de diferentes conhecimentos, maior chance da implantação das decisões e menos oposições, e o aumento da aprendizagem entre os participantes. Os aspectos negativos vinculam-se a decepção dos interessados quando as expectativas não são cumpridas ou incertas, a tomada de decisão em aspectos não importantes e não representados durante o processo e a oposição de implementação de decisões.

No Brasil, a lei 9.433/97 institui a Política Nacional dos Recursos Hídricos (PNRH) criou o Sistema Nacional de Gerenciamento de Recursos Hídricos (SNGRH), e este gerenciamento dos ocorre por meio de decisões locais os quais são denominados de CBHs. Porto e Porto (2008) salientam que esta legislação propicia um destaque ao Brasil, colocando-o entre os países mais avançados no setor de recursos hídricos. O SNGRH, criado pela lei 9433/97, possui os objetivos frente aos recursos hídricos de: a) coordenar a gestão integrada; b) arbitrar os conflitos; c) implementar a PNRH; d) planejar, regular e controlar o uso, a preservação e sua recuperação; e e) promover a cobrança pelo seu uso.

A descentralização desta gestão em nível local ainda carece de uma evolução institucional no país (Porto e Porto, 2008). Estes autores ainda destacam que a lei 9433/97 contribui para um novo paradigma referente a gestão dos recursos hídricos, sendo que a falta desta gestão pode refletir efeitos perversos para toda sociedade brasileira. Cabe destacar que o Brasil ainda estabelece a existência de CBHs transjurisdicionais com integrantes governamentais e da sociedade civil com a finalidade de discutir e decidir questões relativas à gestão da água em cada BH (Perkins, 2011). Estas questões, segundo Datta (2015) abrangem os elementos de solo e conservação da água, incluindo a melhoria de infraestrutura agrícola e social, mercado e acesso ao crédito, e introduz novas tecnologias agrícolas para o desenvolvimento das BHs.

Nesta lógica, este estudo possui como objetivo central analisar se as variáveis intervenientes relacionados aos dados geográficos, demográficos, socioeconômicos e de saneamento possuem correlação com a existência de CBHs. Este propósito justifica-se pela relevância das particularidades quanto aos aspectos geográficos das BHs e frente à distribuição de CBHs no território nacional. Além disto, salienta-se que há carência de um mapeamento comparativo que associe as questões da existência e distribuição dos CBHs entre as Unidades Federativas (UF) brasileiras em relação aos aspectos geográficos, demográficos, socioeconômicos e saneamento.

Kaplowitz e Witter (2008) e Mahmoud et al. (2011) reforçam esta justificativa, destacando que com o prolongamento da escassez hídrica exercendo pressões sobre os sistemas de gestão e distribuição de água existentes torna-se essencial a identificação e análise dos fatores que podem alterar o gerenciamento do consumo para uma eficiente gestão, incluindo as BHs. O sucesso na gestão de BHs, segundo Brody et al. (2005), é realizado por meio de diferentes grupos de stakeholders, mas através de uma melhor compreensão das características socioeconômicas, demográficas e demais variáveis intervenientes.

\section{MATERIAL E MÉTODOS}

Este estudo, quanto à abordagem do problema caracteriza-se como quantitativo, quanto ao procedimento técnico vincula-se a pesquisa documental referente a coleta dos dados e causal em relação a análise das informações coletas. Neste sentido, a pesquisa causal compreende a análise das variáveis intervenientes, por meio da estatística, na explicação de um fenômeno, que neste estudo direciona-se a explicação da existência de $\mathrm{CBH}$ para a gestão dos recursos hídricos. 


\subsection{Coleta e tabulação dos dados}

A coleta dos dados foi realizada pela pesquisa documental secundária abrangendo aspectos geográficos, demográficos, socioeconômicos e de saneamento (Quadro 1).

Quadro 1. Dados coletados e seleção das variáveis.

\begin{tabular}{|lllll|}
\hline Tipo de dados & \multicolumn{1}{c}{ Variáveis } & \multicolumn{1}{c}{ Medida } & \multicolumn{1}{c|}{ Fonte } & Ano base \\
\hline \multirow{5}{*}{ Geográficos } & $-\mathrm{n}^{\circ}$ de municípios por UF & Número & $(\mathrm{CBH}, 2014)$ & 2011 \\
& - Área da BH por UF & $\mathrm{km}^{2}$ & $(\mathrm{CBH}, 2014)$ & 2011 \\
& - CBH por UF & Número & $(\mathrm{CBH}, 2014)$ & 2011 \\
& - Municípios c/CBHs & Número & $(\mathrm{CBH}, 2014)$ & 2011 \\
& - BH por UF & Número & $(\mathrm{CBH}, 2014)$ & 2011 \\
& - Municípios abrangidos por BH & Número & (CBH, 2014) & 2011 \\
\hline \multirow{2}{*}{ Demográficos } & - habitantes por UF & Número & (IBGE, 2013) & 2013 \\
& - habitantes por BH & Número & (CBH, 2014) & 2011 \\
\hline \multirow{5}{*}{ Sócioeconômicos } & - PIB & Percentual & (SIDRA, 2014) & 2011 \\
& - IDHM & Coeficiente & (PNUD et al., 2010) & 2010 \\
& - Renda per capita & Hectares & (SIDRA, 2014) & 2014 \\
& - Índice de degradação ambiental & Reais & (DATASUS, 2014) & 2010 \\
& - Conflitos pela água & Número & (Pinto et al., 2014) & 2014 \\
\hline \multirow{5}{*}{ Saneamento } & - Perda de água na distribuição & Percentual & (TRATABRASIL, 2014) & 2014 \\
& - Tratamento de esgoto & Percentual & (TRATABRASIL, 2014) & 2014 \\
& - Coleta de esgoto & Percentual & (TRATABRASIL, 2014) & 2014 \\
& - Rede de água & Percentual & (TRATABRASIL, 2014) & 2014 \\
\hline
\end{tabular}

Nota: *2014, 2013 e 2012; PIB: Produto Interno Bruto; IDHM: Índice de Desenvolvimento Humano.

Fonte: CBH (2014); IBGE (2013); SIDRA (2014); PNUD et al. (2010); DATASUS (2014); Pinto et al. (2014); CPT (2014); TRATABRASIL (2014).

Esta pesquisa documental foi realizada de março a abril de 2014, em sítios oficiais de internet descritos na coluna fonte do Quadro 1. A seleção das variáveis (Quadro 1) ocorreu em função da disponibilidade de dados e pela aderência aos possíveis fatores que influenciam na existência de CBHs nas diferentes UFs brasileiras. Salienta-se como limitação desta pesquisa documental secundária que podem existir outras variáveis que influenciam no estabelecimento de CBHs nas regiões brasileiras, mas que não estão documentadas ou compreendem dados confiáveis. Após a coleta dos dados, estes foram tabulados por meio de planilhas eletrônicas (Software Microsoft Office Excel), onde na primeira linha inseriu-se a descrição das variáveis por tipo de dados e na primeira coluna da descrição de cada UF. As UFs que integraram este estudo limitam-se as que possuem CBHs.

\subsection{Método de análise}

O método estatístico empregado para a apuração das correlações foi o coeficiente de correlação de Pearson, pois os dados utilizados são paramétricos, e suas medidas escalares são contínuas (Witte e Witte, 2005). O coeficiente de correlação de Pearson está compreendido entre -1 e 1, e a intensidade de correlação é avaliada em: Nula, quando a correlação for 0; Fraca, quando estiver entre 0 e 0,3 ; regular, quando entre 0,31 e 0,6 ; Forte, quando entre 0,61 e 0,9 ; Muito Forte, quando entre 0,91 e 99; e plena, quando apresentar uma correlação igual a 1 (Callegari-Jaques, 2003). O software empregado para correlacionar as variáveis foi o Statistical Package for Social Science for Windows (IBM SPSS). Após apresentar o material e método na sequência apresenta-se os resultados e discussão. 


\section{RESULTADOS E DISCUSSÃO}

Os resultados oriundos da coleta dos dados descrita no Quadro 1, tais como as variáveis geográficas, demográficas, socioeconômicas e de saneamento das UFs brasileiras que possuem CBHs, estão evidenciados na Tabela 1. As UFs estão distribuídas de acordo com as regiões brasileiras: Centro-Oeste (4 UFs), Nordeste (8 UFs), Norte (1 UF), Sudeste (4 UFs) e Sul (3UFs)

Tabela 1. Informações geográficas, demográficas, socioeconômicas e de saneamento.

\begin{tabular}{|c|c|c|c|c|c|c|c|c|c|c|c|c|c|c|c|c|c|c|c|}
\hline \multirow[b]{2}{*}{ UF } & \multicolumn{6}{|c|}{ Geográficos } & \multicolumn{2}{|c|}{ Demográficos } & \multicolumn{7}{|c|}{ Sócio econômicos } & \multicolumn{4}{|c|}{ Saneamento } \\
\hline & 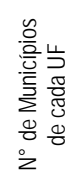 & 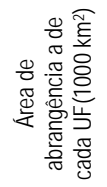 & 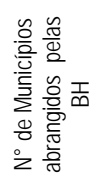 & 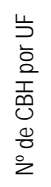 & 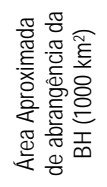 & 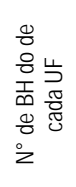 & 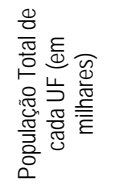 & 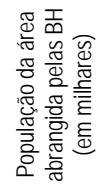 & 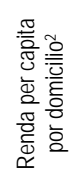 & $\begin{array}{l}\stackrel{\sum}{\infty} \\
\text { 至 }\end{array}$ & $\frac{\vec{m}}{\alpha}$ & 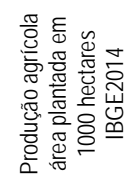 & 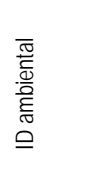 & 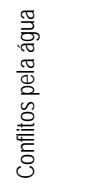 & 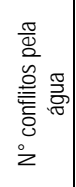 & 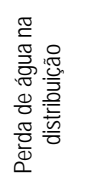 & 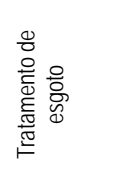 & $\begin{array}{l}\text { 음 } \\
\text { d } \\
0 \\
\frac{0}{0} \\
\frac{\pi}{0} \\
\frac{0}{0}\end{array}$ & 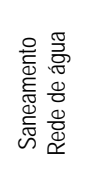 \\
\hline Distrito Federal & 1 & 5,8 & 1 & 3 & 5,8 & 7 & 2789,8 & 2.616 & 1.665 & 0,824 & 164482 & 172,9 & 44,65 & 0,0 & 0 & 37,23 & 66,13 & 82,73 & 98,20 \\
\hline Goiás & 246 & 340,1 & 37 & 3 & 12,4 & 11 & 6434,0 & 2.009 & 785 & 0,735 & 111269 & 6087,3 & 80,14 & 1,0 & 2 & 28,78 & 44,93 & 41,51 & 85,62 \\
\hline Mato Grosso & 141 & 903,4 & 54 & 7 & 109,3 & 27 & 3182,1 & 1.204 & 735 & 0,725 & 71418 & 13542,0 & 68,20 & 4,3 & 6 & 47,17 & 23,85 & 17,72 & 86,23 \\
\hline Mato Grosso do Sul & 79 & 357,1 & 20 & 2 & 41,6 & 15 & 2587,3 & 204 & 785 & 0,729 & 49242 & 4551,7 & 80,19 & 0,0 & 0 & 32,92 & 32,76 & 36,47 & 85,75 \\
\hline Aagoas & 102 & 27,8 & 66 & 5 & 14,4 & 14 & 3300,9 & 3.563 & 421 & 0,631 & 28540 & 571,8 & 39,76 & 1,3 & 2 & 46,12 & 18,52 & 18,83 & 76,46 \\
\hline Bahia & 417 & 564,7 & 366 & 10 & 372,5 & 25 & 15044,1 & 9.785 & 481 & 0,66 & 159869 & 3584,3 & 69,62 & 18,3 & 26 & 41,58 & 46,56 & 31,02 & 77,43 \\
\hline Ceará & 184 & 148,9 & 193 & 10 & 131,5 & 12 & 8778,6 & 7.762 & 446 & 0,682 & 87982 & 1012,1 & 43,57 & 3,0 & 1 & 36,52 & 32,22 & 25,32 & 69,75 \\
\hline Paraíba & 223 & 56,5 & 145 & 3 & 25,7 & 19 & 3914,4 & 2.505 & 462 & 0,658 & 35444 & 332,7 & 54,64 & 1,7 & 0 & 36,18 & 34,02 & 24,54 & 75,6 \\
\hline Pernambuco & 185 & 98,1 & 170 & 7 & 39,6 & 33 & 9208,6 & 5.503 & 509 & 0,673 & 104394 & 803,7 & 32,46 & 6,0 & 8 & 53,69 & 26,38 & 19,68 & 70,89 \\
\hline Piauí & 224 & 251,6 & 94 & 1 & 75,0 & 12 & 3184,2 & 667 & 408 & 0,646 & 24607 & 1443,7 & 72,35 & 0,3 & 0 & 51,82 & 8,30 & 6,64 & 67,12 \\
\hline Rio Grande do Norte & 167 & 52,8 & 85 & 3 & 18,2 & 16 & 3374,0 & 1.831 & 532 & 0,684 & 36103 & 198,7 & 59,81 & 0,7 & 1 & 55,26 & 21,09 & 21,54 & 81,37 \\
\hline Sergipe & 75 & 21,9 & 61 & 3 & 9,5 & 8 & 2195,7 & 1.644 & 508 & 0,665 & 26199 & 284,8 & 50,13 & 0,3 & 0 & 59,27 & 22,39 & 15,25 & 83,05 \\
\hline Tocantins & 139 & 277,7 & 50 & 7 & 56,2 & 30 & 1478,2 & 557 & 572 & 0,699 & 18059 & 1033,2 & 78,40 & 2,0 & 2 & 34,34 & 17,65 & 14,71 & 75,45 \\
\hline Espírito Santo & 78 & 46,1 & 75 & 13 & 26,2 & 14 & 3839,4 & 2.312 & 795 & 0,74 & 97693 & 131,0 & 53,23 & 3,0 & 5 & 34,39 & 32,36 & 41,82 & 80,9 \\
\hline Minas Gerais & 853 & 586,5 & 1083 & 38 & 557,5 & 43 & 20593,4 & 18.779 & 733 & 0,731 & 386156 & 4329,4 & 55,73 & 14,0 & 26 & 33,46 & 32,76 & 74,22 & 86,97 \\
\hline Rio de Janeiro & 92 & 43,8 & 110 & 10 & 40,5 & 10 & 16369,2 & 15.660 & 993 & 0,761 & 462376 & 122,0 & 55,19 & 5,7 & 7 & 30,82 & 34,32 & 62,59 & 89,15 \\
\hline São Paulo & 645 & 248,2 & 829 & 22 & 248,4 & 22 & 43663,7 & 39.809 & 1.037 & 0,783 & 1349465 & 7503,7 & 43,92 & 1,0 & 0 & 34,34 & 53,34 & 87,36 & 95,85 \\
\hline Paraná & 399 & 199,3 & 476 & 11 & 183,5 & 16 & 10997,5 & 10.244 & 871 & 0,749 & 239366 & 10587,9 & 55,48 & 2,0 & 3 & 33,35 & 63,75 & 60,00 & 91,15 \\
\hline Rio Grande do Sul & 295 & 95,7 & 672 & 25 & 271,2 & 25 & 6634,3 & 10.603 & 940 & 0,746 & 263633 & 8871,2 & 42,31 & 2,0 & 2 & 37,23 & 12,58 & 29,15 & 84,06 \\
\hline Santa Catarina & 497 & 281,7 & 321 & 17 & 78,0 & 24 & 11164,0 & 5.129 & 967 & 0,774 & 169050 & 1545,7 & 19,79 & 2,3 & 3 & 33,71 & 19,58 & 16,03 & 86,02 \\
\hline
\end{tabular}

Nota: ${ }^{1}$ Produto Interno Bruto de cada Estado, em 2011, valores em (R\$1.000,00).

2 Renda per capita por domicílio corresponde à média mensal, em 2010, e em R\$1.000,00.

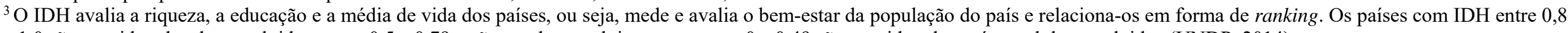
e 1,0 são considerados desenvolvidos, entre 0,5 e 0,79 estão em desenvolvimento, e entre 0 e 0,49 são considerados países subdesenvolvidos (UNDP, 2014).

Rev. Ambient. Água vol. 12 n. 2 Taubaté - Mar. / Apr. 2017 
A correlação de Pearson realizou-se com base na Tabela 1, e seus resultados estão evidenciados na Tabela 2. As correlações revelam que existe uma forte correlação, conforme classificação de Callegari-Jaques (2003), entre o número de CBH com: o número de municípios de cada UF $\left(p=0,806^{* *}\right)$; a população total de cada UF (habitantes) $\left(p=0,613^{* *}\right)$; a área aproximada de abrangência da $\mathrm{BH}\left(p=0,826^{* *}\right)$; o número de municípios abrangidos pelas $\mathrm{BH}\left(p=0,919^{* *}\right)$; e a população da área abrangida pelas $\mathrm{BH}\left(p=0,664^{* *}\right)$. Estes resultados apontam que o número de $\mathrm{CBHs}$ existentes podem ser relacionados as regiões com maior concentração populacional.

Tabela 2. Correlação de Pearson entre variáveis socioeconômicas e geográficas de BHs brasileiras.

\begin{tabular}{|c|c|c|c|c|c|c|c|c|c|c|c|c|c|c|c|c|c|c|c|}
\hline & 1 & 2 & 3 & 4 & 5 & 6 & 7 & 8 & 9 & 10 & 11 & 12 & 13 & 14 & 15 & 16 & 17 & 18 & 19 \\
\hline 1 & 1 & & & & & & & & & & & & & & & & & & \\
\hline 2 & 0,416 & 1 & & & & & & & & & & & & & & & & & \\
\hline 3 & $0,730 * *$ & 0,163 & 1 & & & & & & & & & & & & & & & & \\
\hline 4 & $0,575^{* *}$ & 0,047 & $0,949 * *$ & 1 & & & & & & & & & & & & & & & \\
\hline 5 & 0,034 & $-0,092$ & 0,241 & 0,405 & 1 & & & & & & & & & & & & & & \\
\hline 6 & 0,19 & 0,036 & 0,363 & $0,498^{*}$ & $0,942 * *$ & 1 & & & & & & & & & & & & & \\
\hline 7 & $0,806^{* *}$ & 0,259 & $0,613 * *$ & $0,543 *$ & 0,222 & 0,371 & 1 & & & & & & & & & & & & \\
\hline 8 & $0,834 * *$ & $0,515^{*}$ & $0,567 * *$ & 0,416 & 0,008 & 0,101 & $0,826^{* *}$ & 1 & & & & & & & & & & & \\
\hline 9 & $0,686^{* *}$ & 0,077 & $0,975 * *$ & $0,964 * *$ & 0,28 & 0,382 & $0,664 * *$ & $0,585 * *$ & 1 & & & & & & & & & & \\
\hline 10 & $0,909 * *$ & 0,258 & $0,733 * *$ & $0,650 * *$ & 0,149 & 0,269 & $0,919 * *$ & $0,887 * *$ & $0,775^{* *}$ & 1 & & & & & & & & & \\
\hline 11 & $0,638 * *$ & $0,541^{*}$ & 0,303 & 0,163 & $-0,168$ & $-0,036$ & $0,649 * *$ & $0,661 * *$ & 0,278 & $0,611 * *$ & 1 & & & & & & & & \\
\hline 12 & $-0,444^{*}$ & $-0,056$ & $-0,4$ & $-0,451^{*}$ &,$- 602 * *$ & ก & $-0,618^{* *}$ & $-0,34$ & $-0,44$ & $-0,543 *$ & $-0,093$ & 1 & & & & & & & \\
\hline 13 & 0,343 & $0,637 * *$ & 0,257 & 0,299 & 0,19 & 0,307 & 0,307 & 0,403 & 0,283 & 0,397 & 0,286 & $-0,474 *$ & 1 & & & & & & \\
\hline 14 & $-0,294$ & $-0,111$ & $-0,314$ & $-0,33$ & $-0,474 *$ & ก $500 * *$ & $-0,377$ & $-0,233$ & $-0,32$ & $-0,284$ & $-0,056$ & $0,517^{*}$ & $-0,2$ & 1 & & & & & \\
\hline 15 & 0,169 & 0,003 & 0,396 & 0,403 & $0,574 * *$ & $0,537^{*}$ & 0,032 & 0,153 & 0,373 & 0,171 & $-0,204$ & $-0,27$ & 0,216 & $-0,450^{*}$ & 1 & & & & \\
\hline 16 & 0,389 & $-0,022$ & $0,641 * *$ & $0,702 * *$ & $0,722 * *$ & $0,723 * *$ & $0,456^{*}$ & 0,381 & $0,681 * *$ & $0,503 *$ & $-0,004$ & $-0,507^{*}$ & 0,195 & $-0,543 *$ & $0,781^{* *}$ & 1 & & & \\
\hline 17 & 0,223 & 0,083 & 0,408 & $0,544^{*}$ & $0,848 * *$ & $0,851 * *$ & 0,303 & 0,152 & 0,441 & 0,304 & $-0,099$ & $-0,641 * *$ & 0,399 & $-0,42$ & $0,630 * *$ & $0,777 * *$ & 1 & & \\
\hline 18 & $-0,178$ & 0,429 & $-0,246$ & $-0,229$ & $-0,224$ & $-0,190$ & $-0,338$ & $-0,005$ & $-0,274$ & $-0,266$ & $-0,080$ & 0,361 & 0,215 & $-0,108$ & 0,027 & $-0,081$ & $-0,095$ & 1 & \\
\hline 19 & $0,502 *$ & $0,509^{*}$ & 0,315 & 0,078 & $-0,184$ & $-0,148$ & $0,465^{*}$ & $0,738 * *$ & 0,259 & $0,449 *$ & $0,582 * *$ & $-0,006$ & 0,067 & $-0,076$ & 0,126 & 0,143 & $-0,098$ & 0,071 & 1 \\
\hline
\end{tabular}

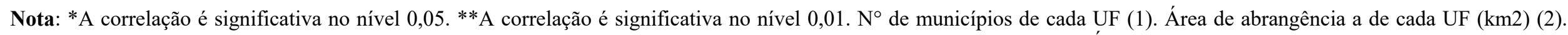

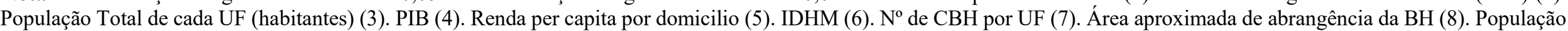

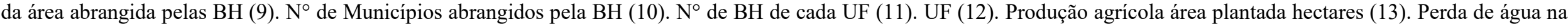
distribuição (14). Tratamento de esgoto (15). Coleta de esgoto (16). Rede de água (17). ID ambiental (18). Conflitos pela água (19). 
O aumento da concentração populacional em determinada área, pode gerar um conflito pela distribuição equitativa de água da $\mathrm{BH}$, pois esta abastece as residências e indústrias urbanas, bem como a produção agrícola rural, quando for o caso. Barreto et al. (2010) destacam que a água é utilizada como estratégia na administração de conflitos e proteção ambiental, o que justificaria o estabelecimento da gestão de $\mathrm{BHs}$ por meio de $\mathrm{CBH}$, pois busca o equilíbrio entre a demanda e o consumo humano, dessedentação animal e irrigação. Neste sentido, Mahmoud et al. (2011) em seu estudo revela que o fator crescimento populacional obriga os gestores de $\mathrm{BHs}$ e os stakeholders a tomarem decisões críticas sob diferentes graus de incertezas. Gaddis et al. (2010) salientam que a gestão de BHs por CBHs afeta e é afetada pela sociedade, principalmente em relação à poluição.

A institucionalização de CBHs possui correlação moderada com regiões em que há a coleta de esgoto $\left(p=0,456^{*}\right)$, porém com os demais aspectos de saneamento relacionados a perda de água na distribuição, tratamento de esgoto e a disponibilidade da rede de água não apresentou correlação significativa. Entretanto, esse achado não corrobora com estudos de Liu et al. (2008) que revelam que a gestão hídrica, principalmente em regiões de pobreza onde não há investimentos em recursos naturais, é necessária para resolver adequadamente os problemas com a distribuição de água. Os resultados de Abers e Jorge (2005) afirmam que a criação de comitês, na maioria dos casos, relaciona-se na solução de problemas na $\mathrm{BH}$, tais como, os ambientais, conflitos entre usos da água e a incidência de eventos críticos.

Esta reflexão corrobora com o que é postulado por Said et al. (2006) afirmando que a deterioração da qualidade e escassez de água são os propulsores do desenvolvimento de estratégias de gestão das BHs por meio de CBHs. E ainda salientam que um dos propósitos da gestão de BHs vincula-se à compreensão das ações necessárias no restabelecimento da qualidade da água. Campos (2004) exemplifica que a criação das Bacias Hidrográficas dos Rios Piracicaba, Capivari e Jundiaí/SP ocorreu em função da degradação e da escassez de águas comprometerem o desenvolvimento socioeconômico desta região, portanto o $\mathrm{CBH}$ teve como objetivo a preservação e restauração ecológica. Os gestores, diante dos diversos interesses e necessidades dos usuários, podem ter dificuldades para compreender os diferentes objetivos destes grupos (Kaplowitz e Witter, 2008). Portanto, a gestão de BHs destina-se a proteger os recursos hídricos superficiais e subterrâneos, e para isto, centra-se na gestão integrada e participativa dos recursos da água e do solo (Ferreyra et al., 2008).

A quantidade de $\mathrm{CBHs}$ também apresentou uma correlação significativa moderada $\left(p=0,543^{*}\right)$ com o PIB da cada UF, conforme classificação de Callegari-Jaques (2003), mostrando que quanto maior o PIB do estado, maior é o número de CBHs estabelecidos e funcionando. Neste sentido, esta informação revela que o processo de instalação de CBHs tem uma relação com o PIB, pois na medida em que ocorre um crescimento econômico, poderá refletir-se na disponibilidade da água.

Sanchez-Roman et al. (2009) salientam que o desenvolvimento econômico demanda enormes quantidades de água. Said et al. (2006) corrobora com esta afirmação, destacando que o aumento da gestão hídrica e a participação pública, nestes comitês, está vinculada ao estímulo econômico. Para Falkenmark e Molden (2008) o crescimento econômico da população implica no crescimento da demanda pela água e de alimentos. A região Sudeste do Brasil enfrenta os principais problemas de recursos hídricos voltados à questão da escassez pela poluição e o uso excessivo das águas nas áreas urbanas e industrializadas, seus conflitos de uso e as constantes inundações (Porto et al., 1999). Barreto et al. (2010) acrescenta que a associação entre a falta hídrica e a exploração econômica desordenada provocam a degradação e desertificação do solo, e a redução de espécies de vegetais e animais.

O número de CBHs com a variável UF também apresentou uma correlação significativa forte $\left(p=-0,618^{* *}\right)$. Este resultado revela que as regiões brasileiras do sul e sudeste possuem 
maior quantidade de CBHs e, portanto, maior participação pública para a realização da gestão das BHs em relação às regiões do Nordeste, Centro Oeste e Norte. Destaca-se que para a região Norte as informações acerca de CBHs foi de difícil obtenção quando comparada a outras UFs, apesar desta possuir a maior BH brasileira, a do Amazonas com 7,05 milhões de km² (Da Costa et al., 2013).

Este resultado, das regiões do sul e sudeste do Brasil apresentarem maior quantidade de CBHs, também é explicado por Abers e Jorge (2005) que apontam que os governos estaduais foram os responsáveis pela criação de $\mathrm{CBHs}$ em todas as BHs dos estados de São Paulo e do Rio Grande do Sul. Entretanto, os mesmos autores ainda destacam que a criação de CBHs nos estados de Minas Gerais e no Ceará, ocorreu de forma mais seletiva, ou seja, sua criação ocorreu nas BHs de maior interesse para os órgãos governamentais. Watanabe et al. (2014) afirmam que a formação de CBHs no Rio Grande do Sul foi influenciada por decisões locais e consideradas como particulares em cada região do estado.

A quantidade de CBHs também apresentou uma correlação moderada $\left(p=0,465^{*}\right)$ com a quantidade de conflitos pela água em cada UF. Braga et al. (2008) também apontam que nas regiões do Brasil, onde há intensa ocupação do território, tem gerado conflitos pelo uso da água. Portanto, a intensa ocupação populacional em determinada região promove conflitos em relação ao uso das águas pela sua quantidade e qualidade, o que exige a necessidade de institucionalização de um CBH para gerenciar os conflitos entre os diversos usuários.

\section{CONCLUSÃO}

O escopo principal deste estudo foi verificar se as variáveis intervenientes relacionadas aos dados geográficos, demográficos, socioeconômicos e de saneamento possuem correlação com a criação de $\mathrm{CBHs}$. O estabelecimento de $\mathrm{CBH}$ vincula-se com as $\mathrm{BHs}$ com os conflitos no uso da água pelos diversos stakeholders, assim como, também com o tamanho populacional e crescimento econômico (PIB).

A existência de CBHs no Brasil, advém do vasto aglomerado de habitantes que necessitam de grande quantidade de água, para consumo humano e animal, produção de alimentos, de produtos na indústria, recreação, entre outros. Esta por sua vez, cria uma necessidade de demanda muito elevada e pode provocar até a escassez de água, e também, ao mesmo tempo, a degradação através dos resíduos orgânicos sólidos e líquidos, químicos, erosão do solo, entre outros. A partir desta escassez e pela falta de qualidade de água, pode ocorrer uma estagnação e ou decrescimento da situação econômica.

Portanto, é essencial na gestão de BHs por meio de CBHs a participação pública local e específica, pois estes se relacionam tanto na criação e no sucesso da implantação de estratégias principalmente vinculados a degradação ambiental e conflitos de múltiplos usos. Considera-se vital a gestão dos recursos hídricos, especialmente num país com tantas particularidades e dimensões continentais como o Brasil. E, ainda, recomenda-se que nas BHs estruturem-se CBHs antes de sua degradação, pois neste caso, a gestão torna-se mais eficiente e os conflitos mais amenos, contribuindo assim, no estabelecimento da resiliência do sistema ambiental.

\section{AGRADECIMENTOS}

Ao Centro Universitário Univates, Universidade Federal do Rio Grande do Sul e ao Programa de Pós-Graduação em Qualidade Ambiental da Universidade Feevale pelo apoio à pesquisa.

\section{IPABH}




\section{REFERÊNCIAS}

ABERS, R. N.; JORGE K. D. Descentralização da Gestão da Água: Por que os comitês de bacia estão sendo criados? Ambiente \& Sociedade, v. 8, p. 1-26, 2005.

ANDRÉ, P.; ENSERIK, B.; CONNOR, D.; CROAL, P. Public participation: international best practice principles Fargo: . International Association for Impact, 2006.

BARRETO, J. F.; DANTAS NETO, J.; FARIAS, S. A. R. Avaliação socioeconômica e hídrica dos municípios da sub-bacia hidrográfica do rio Taperoá, PB. Qualit@s Revista Eletrônica, v. 9, n. 1, p. 1-13, 2010. http://dx.doi.org/10.18391/qualitas.v9i1.585

BRAGA, P. F. B.; FLECHA, R.; PENA, S. D.; KELMAN, J. Pacto federativo e gestão de águas. Estudos Avançados, v. 22, n. 63, p. 17-42, 2008. http://dx.doi.org/10.1590/S0103-40142008000200003

BRODY, S. D.; HIGHFIELD, W.; PECK, M. B. Exploring the mosaic of perceptions for water quality across watersheds in San Antonio, Texas. Landscape and Urban $\begin{array}{llllll}\text { Planning, } & \text { v. } 73, \quad \text { n. } 2 / 3, \quad \text { p. 200-214, }\end{array}$ http://dx.doi.org/10.1016/j.landurbplan.2004.11.010CALLEGARI-JACQUES， S. M. Bioestatística: princípios e aplicações. Porto Alegre: Artmed, 2003. 256 p.

CAMPOS, V. N. D. O. Novos arranjos, velhos problemas. Revista Electrónica de la REDLACH, v. 1, n. 1, p. 63-68, 2004.

COMITÊS DE BACIAS HIDROGRÁFICAS. Website. 2014. Disponível em: http://www.cbh.gov.br/. Acesso em: 17 set. 2014.

COMISSÃO PASTORAL DA TERRA - CPT. Conflitos pela água. 2014. Disponível em: https://goo.gl/SKnID1. Acesso em: 11 dez. 2014.

DA COSTA, L. J.; MOURA, R. G.; OLIVEIRA, J. M. Análise multitemporal das mudanças do uso do solo ocorridas entre os anos 1986 e 2010 na bacia do rio dos Sinos-Rio Grande Sul-Brasil. In: SIMPÓSIO BRASILEIRO DE SENSORIAMENTO REMOTO - SBSR, 16., 2013, Foz do Iguaçu. Anais... São José dos Campos: INPE, 2013. p. 7438-7444.

DATTA, N. Evaluating Impacts of Watershed Development Program on Agricultural Productivity, Income, and Livelihood in Bhalki Watershed of Bardhaman District, West Bengal. World Development, v. 66, p. 443-456, 2015. http://dx.doi.org/10.1016/j.worlddev.2014.08.024

DATASUS. Indicadores socioeconômicos. 2014. Disponível em: http://tabnet.datasus.gov.br/cgi/tabcgi.exe?idb2011/b08a.def. Acesso em: 12 set. 2014.

FALKENMARK, M.; MOLDEN, D. Wake up to realities of river basin closure. International Journal of Water Resources Development, v. 24, p. 201-215, 2008. http://dx.doi.org/10.1080/07900620701723570

FERREYRA, C.; LOË, R. C.; KREUTZWISER, R. D. Imagined communities, contested watersheds: Challenges to integrated water resources management in agricultural areas. Journal of Rural Studies, v. 24, n. 3, p. 304-321, 2008. http://dx.doi.org/10.1016/j.jrurstud.2007.11.001 
GADDIS, E. J. B.; FALK, H. H.; GINGER, C.; VOINOV, A. Effectiveness of a participatory modeling effort to identify and advance community water resource goals in St. Albans, Vermont. Environmental Modelling \& Software, v. 25, n. 11, p. 1428-1438, 2010. http://dx.doi.org/10.1016/j.envsoft.2009.06.004

HAJKOWICZ, S. Allocating scarce financial resources across regions for environmental management in Queensland, Australia. Ecological Economics, v. 61, n. 2-3, p. 208-216, 2007. http://dx.doi.org/10.1016/j.ecolecon.2006.10.011

HOPE, R. A. Evaluating Social Impacts of Watershed Development in India. World $\begin{array}{lllllll}\text { Development, } & \text { v. } 35, \quad \text { n. } & 8, & \text { p. }\end{array}$ http://dx.doi.org/10.1016/j.worlddev.2007.04.006

INSTITUTO BRASILEIRO DE GEOGRAFIA E ESTATÍSTICA - IBGE. Website. 2013. Disponível em: http://www.ibge.gov.br. Acesso em: 15 set. 2014.

KAPLOWITZ, M. D.; WITTER, S. G. Agricultural and residential stakeholder input for watershed management in a mid-Michigan watershed. Landscape and Urban Planning, v. 84, n. 1, p. 20-27, 2008. http://dx.doi.org/10.1016/j.landurbplan.2007.06.004

KORFF, Y. V.; D'AQUINO, P.; DANIELL, K. A.; BIJLSMA, R. Designing participation processes for water management and beyond. Ecology and Society, v. 15, n. 3, p. 1-24, 2010. http://www.ecologyandsociety.org/vol15/iss3/art1/

LIU, B. M.; ABEBE, Y.; MCHUGH, O. V., COLLICK, A. S.; GEBREKIDAN, B.; STEENHUIS, T. S. Overcoming limited information through participatory watershed management: Case study in Amhara, Ethiopia. Physics and Chemistry of the Earth, Parts A/B/C, v. 33, n. 1-2, p. 13-21, 2008. http://dx.doi.org/10.1016/j.pce.2007.04.017

MAHMOUD, M. I.; GUPTA, H. V.; RAJAGOPAL, S. Scenario development for water resources planning and watershed management: Methodology and semi-arid region case study. Environmental Modelling \& Software, v. 26, n. 7, p. 873-885, 2011. http://dx.doi.org/10.1016/j.envsoft.2011.02.003

MALHEIROS, T. F.; PROTA, M. G.; RINCÓN, M. A. P. Participação comunitária e implementação dos instrumentos de gestão da água em bacias hidrográficas. Revista Ambiente \& Água, v. 8, n. 1, p. 98-118, 2013. http://dx.doi.org/10.4136/ambi-agua.970

PERKINS, P. E. Public participation in watershed management: International practices for inclusiveness. Physics and Chemistry of the Earth, Parts A/B/C, v. 36, n. 5-6, p. 204212, 2011. http://dx.doi.org/10.1016/j.pce.2010.02.004

PINTO, N. G. M.; CORONEL, D. A.; CONTE, B. P. Raqueamento da degradação ambiental nos estados e regiões brasileiras. Revista Reunir, v. 6, n. 2, p. 68-82, 2016. http://dx.doi.org/10.18696/reunir.v6i2.374.

PORTO, M.; LA LAINA PORTO, R.; AZEVEDO, L. G. T. A participatory approach to watershed management: The Brazilian system. JAWRA Journal of the American Water Resources Association, v. 35, n. 3, p. 675-683, 1999. http://dx.doi.org/10.1111/j.1752-1688.1999.tb03623.x

PORTO, M. F. A.; PORTO, R. La L. Gestão de bacias hidrográficas. Estudos Avançados, V. 22, n. 63, p. 43-60, 2008. https://dx.doi.org/10.1590/S0103-40142008000200004 
PROGRAMA DAS NAÇÕES UNIDAS PARA O DESENVOLVIMENTO - PNUD; INSTITUTO DE PESQUISA ECONÔMICA APLICADA - IPEA; FUNDAÇÃO JOÃO PINHEIRO. Atlas do desenvolvimento humano no Brasil: ranking - todo o

Brasil. 2010. Disponível em: http://atlasbrasil.org.br/2013/pt/ranking. Acesso em: 15 set. 2014.

SANCHEZ-ROMAN, R. M.; FOLEGATTI, M. V.; ORELLANA GONZALEZ, A. M. G.; SILVA, R. T. da. Dynamic systems approach assess and manage water resources in river

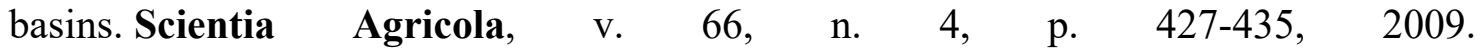
http://dx.doi.org/10.1590/S0103-90162009000400001

SAID, A.; SEHLKE, G.; STEVENS, D.K.; GLOVER, T.; SORENSEN, D.; WALKER, W.; HARDY, T. Exploring an innovative watershed management approach: From feasibility to sustainability. Energy, v. 31, n. 13, p. 2373-2386, 2006. http://dx.doi.org/10.1016/j.energy.2006.02.002

SISTEMA IBGE DE RECUPERAÇÃO AUTOMÁTICA - SIDRA. Website. 2014. Disponível em: http://www.sidra.ibge.gov.br/. Acesso em: 10 set. 2014.

SILVA, N. S.; RIBEIRO, C. A. A. S.; BARROSO, W. R.; RIBEIRO, P. E. Á.; SOARES, V. P.; SILVA, E. Sistema de Otto-codificação modificado para endereçamento de redes hidrográficas. Revista Árvore, v. 32, n. 5, p. 891-897, 2008. http://producao.usp.br/handle/BDPI/4386

TRATABRASIL. Saneamento no Brasil. 2014. Disponível em: http://www.tratabrasil.org.br/saneamento-no-brasil. Acesso em: 15 dez. 2014.

UNITED NATIONS DEVELOPMENT PROGRAMME - UNDP. Human Development Index (HDI). 2014. Disponível em: http://hdr.undp.org/en/content/human-developmentindex-hdi. Acesso em: 01 set. 2014.

WATANABE, M. M.; MADRUGA, L. R. G.; YAMAGUCHI, C. K.; VIEIRA, A. C. P.; JENOVEVA-NETO, R. Decision Making and Social Learning: the Case of Watershed Committee of the State of Rio Grande do Sul, Brazil. Water resources management, v. 28, n. 11, p. 3815-3828, 2014. http://dx.doi.org/10.1007/s11269-014-0711-2

WITTE, R. S.; WITTE, J. S. Estatística. 7. ed. Rio de Janeiro: LTC, 2005. 486 p. 